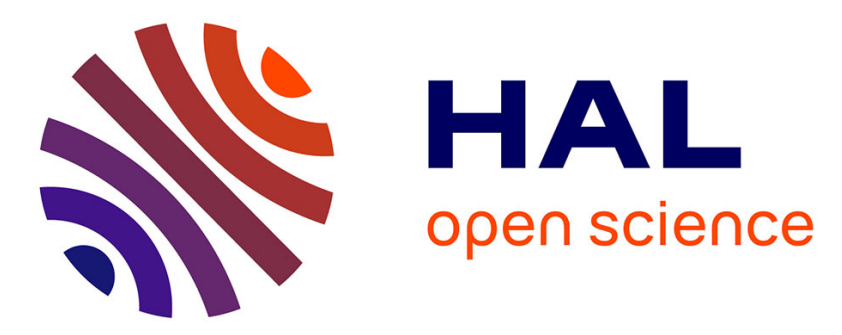

\title{
Revisiting the evolution of the somatostatin family: already five genes in the gnathostome ancestor
}

Hervé Tostivint, Anne-Laure Gaillard, Sylvie Mazan, Guillaume Pézeron

\section{To cite this version:}

Hervé Tostivint, Anne-Laure Gaillard, Sylvie Mazan, Guillaume Pézeron. Revisiting the evolution of the somatostatin family: already five genes in the gnathostome ancestor. General and Comparative Endocrinology, 2019. mnhn-02276574

\section{HAL Id: mnhn-02276574}

\section{https://hal-mnhn.archives-ouvertes.fr/mnhn-02276574}

Submitted on 2 Sep 2019

HAL is a multi-disciplinary open access archive for the deposit and dissemination of scientific research documents, whether they are published or not. The documents may come from teaching and research institutions in France or abroad, or from public or private research centers.
L'archive ouverte pluridisciplinaire HAL, est destinée au dépôt et à la diffusion de documents scientifiques de niveau recherche, publiés ou non, émanant des établissements d'enseignement et de recherche français ou étrangers, des laboratoires publics ou privés. 


\title{
Revisiting the evolution of the somatostatin family: already five genes in the gnathostome ancestor
}

\author{
Hervé Tostivint $^{\mathrm{a}}$, Anne-Laure Gaillard $^{\mathrm{a}}$, Sylvie Mazan ${ }^{\mathrm{b}}$, Guillaume Pézeron $^{\mathrm{a}}$
}

a Physiologie moléculaire et adaptation UMR 7221 CNRS and Muséum National d'Histoire Naturelle, Paris, France

b Biologie intégrative des organismes marins, UMR 7232 CNRS, Observatoire Océanologique, Sorbonne Université, Banyuls-sur-Mer, France

(Correspondence should be addressed to H. Tostivint; E-mail: htostivi@mnhn.fr)

Keywords: somatostatin, neuropeptides, evolution, multigenic family, cartilaginous fish, chondrichthyes, elasmobranchii, holocephali, vertebrates 


\begin{abstract}
The somatostatin (SST) family members are a group of neuropeptides that are best known for their role in the regulation of growth, development and metabolism. The occurrence of six paralogous SST genes named SST1, SST2, SST3, SST4, SST5 and SST6 has been reported in vertebrates. It has been proposed that $S S T 1, S S T 2$ and SST5 arose in 2R from a common ancestral gene. SST3 and SST6 would have been subsequently generated by tandem duplications of the SST1 and SST2 genes respectively, at the base of the actinopterygian lineage. SST4 is thought to have appeared more recently from $S S T 1$, in teleost-specific 3R.

In order to gain more insights into the SST gene family in vertebrates, we sought to identify which paralogs of this family are present in cartilaginous fish. For this purpose, we first searched the recently available genome and transcriptome databases from the catshark Scyliorhinus canicula. In a previous study, three $S$. canicula SST genes, called at that time SSTa, SSTb and SSTc, were identified and proposed to correspond to SST1, SST5 and SST2 respectively. In the present work, two additional SST genes, called SSTd and SSTe, were found in S. canicula plus two other chondrichtyan species, elephant shark (Callorhinchus milii) and whale shark (Rhincodon typus). Phylogeny and synteny analyses were then carried out in order to reveal the evolutionary relationships of SSTd and SSTe with other vertbrates SSTs. We showed that $S S T d$ and $S S T e$ correspond to $S S T 2$ and $S S T 3$ respectively, while $S S T c$ corresponds to SST6 and not to SST2 as initially proposed. Our investigations in other vertebrate species also led us to find that the so-called SST2 gene in chicken, lungfish, sturgeons and teleosts actually corresponds to SST6. Conversely, the so-called SST6 gene in actinopterygians corresponds to SST2.

Taken together, our results suggest that: i) SST3 and SST6 were already present in the gnathostome ancestor, much earlier than previously thought; ii) SST6 was also present in the tetrapod ancestor and still occurs in living birds; with this respect, it is likely that SST6 was independently lost several times during evolution: in euteleosts, amphibians, squamates and mammals; iii) SST2, SST3 and SST5 were probably lost in euteleosts, sarcopterygians and tetrapods, respectively.
\end{abstract}




\section{Introduction}

Somatostatin (currently known as somatostatin 1 or SST1) was originally isolated from ovine hypothalamus and found to inhibit the release of growth hormone from the rat pituitary (Brazeau et al. 1973). It has been subsequently shown that SST1 is widely distributed in the central nervous system and in various peripheral organs where it plays important roles in the regulation of growth, development and metabolism (Günther et al. 2018). The occurrence of SST1 was reported in all vertebrate species investigated so far. Moreover, most species were shown to possess additional SST forms. In gnathostomes (jawed vertebrates), it was demonstrated that all these SST variants are the products of at least six paralogous genes named SST1, SST2 (also known as Cortistatin in mammals) SST3, SST4, SST5 and SST6 (de Lecea et al. 1996, Liu et al. 2010, Tostivint et al. 1996, 2008, 2013, 2014). SST-related peptides are thought to act through at least six SST receptor subtypes (Ocampo Daza et al. 2012). In lampreys, three SST genes have characterized but their phylogenetic status is still unclear (Tostivint et al., 2016), while in the hagfish Myxine glutinosa, the occurrence of only one SST gene, encoding a SST1-like peptide, is attested to date (Conlon et al., 1988).

It is currently well accepted that the evolution of the SST gene family, as that of many other neuropeptides (Elphick et al. 2018), was strongly shaped by the different rounds of wholegenome duplications that took place in the vertebrate history, especially the two basal rounds of vertebrate genome doubling (2R) and the teleost fish-specific genome doubling (3R) (Dehal \& Boore 2005, Putnam et al. 2008, Sacerdot et al. 2018). Thus, SST1, SST2 and SST5 seem to have been generated during 2R from a common ancestral gene, while SST4 is likely to have appeared during 3R from SST1. Impact of 2R on the early evolution of SST gene family is notably supported by synteny analysis showing that $S S T$ genes are located on a same tetraparalogon that contains paralogous genes of at least ten other families (Liu et al. 2010, Tostivint et al., 2013, 2014). For their part, SST3 and SST6 would have been independently generated by tandem duplications of SST1 and SST2 respectively, at the base of the actinopterygian lineage (Liu et al. 2010, Tostivint et al. 2004, 2008). Some of these genes could have been subsequently lost explaining their absence in some lineages. For example, SST5 is believed to have been lost in tetrapods.

Cartilaginous fish (chondrichthyans) are of particular interest in evolutionary studies because of their key phylogenetic position at the base of the gnathostome lineage. They consist of two major groups: elasmobranchii (sharks and skates/rays) and holocephali (chimaeras). Until 2013, SST-related peptides were characterized in only a very few species of cartilaginous fish: SST1 from the ray Torpedo marmorata (Conlon et al. 1985), a SST1-like peptide exhibiting a serine residue at position 5 from the ratfish Hydrolagus colliei (Conlon 1990), a SST2-like sequence from the elephant shark Callorhinchus milii (Tostivint et al. 2008) and finally a totally atypical SST-related sequence from both the little skate Leucoraja erinacea and C. milii (Liu et al. 2010). In 2013, three SST genes, called SSTa, SSTb and SSTc, were identified in the common dogfish (today referred to as small-spotted catshark, Scyliorhinus canicula) and proposed to correspond to SST1, SST5 and SST2, respectively (Quan et al. 2013). Surprisingly, none of these genes appeared as the apparent ortholog of the atypical SST-like gene found in $C$. milii and $L$. erinacea. Nevertheless, these findings were regarded as in good agreement with the model described above since they support the existence of these three SST genes in the gnathostome ancestor (Liu et al. 2010, Tostivint et al. 2013, 2014). However, due to the lack of a fully sequenced and assembled $S$. canicula genome, this view could not be confirmed (Quan et al. 2013).

The first goal of the present study was to determine the complete repertoire of the SST gene family in cartilaginous fish. For this purpose, we searched the newly available $S$. canicula genome and transcriptome databases (unpublished data). We also took the opportunity to explore or reexplore the genome and transcriptome databases from two additional species, the 
whale shark, Rhincodon typus (Read et al. 2017) and the elephant shark, C. milii (Venkatesh et al. 2014). We found two novel S. canicula SST genes that we propose to provisionally call SSTd and SSTe (according to the nomenclature initially proposed in Quan et al. 2013). S. canicula SSTd was shown to be the ortholog of the L. erinacea and C. milii atypical SST-like gene abovementioned, while $S$. canicula SSTe appeared as a totally new SST gene. In both R. typus and $C$. milii, four SST genes were found that correspond SSTa, SSTc, SSTd and SSTe.

Taking advantage of the genome assembly of various representative species from other vertebrate groups, we then carried out phylogeny and synteny analyses in order to reveal the evolutionary relationships of chondrichthyan SSTd and SSTe with other vertebrates SSTs. From our investigations, it appeared that SSTd and SSTe correspond to SST2 and SST3, respectively, while SSTc corresponds to SST6 but not to SST2 as initially proposed (Quan et al. 2013).

The major implication of these results is that the repertoire of the SST gene family in the gnathostome ancestor was already composed of five paralogs namely SST1, SST2, SST3, SST5 and SST6. Our observations also led us to revisit the phylogenetic status of SST2 and SST6. As a result, the nomenclature of these genes had to be corrected in some species.

\section{Material and methods}

\section{Nomenclature}

In this article, S. canicula SSTs, as well as their counterparts from other chondrychthyans, will be first described as SSTa-SSTe, according to the provisionnal nomenclature proposed by Quan et al. (2013), without prejudice to their evolutionary relationships with other vertebrates SSTs. Thereafter, in the light of the phylogeny and synteny data, they will be renamed according to the phylogenetic nomenclature, SST1-SST6, initially introducted by Tostivint et al. (2008) then extended by Liu et al. (2010). Nevertheless, the correspondence bewteen the two nomenclatures will be regularly reminded throughout the article as follows: SSTa $=S S T 1 ; S S T b=S S T 5$; SSTc $=$ SST6; $S S T d=$ SST2 and SSTe $=$ SST3. Note that in Quan et al. (2013) SSTc was initially but mistakenly referred to as $S S T 2$.

\section{Identification of $S S T d$ and $S S T e$ sequences from cartilaginous fish}

S. canicula SSTd and SSTe genes were sought by TBLASTN (Altschul et al. 1990) using the $C$. milii and L. erinacea SST2-like sequences (AGCKLFFWKTFSHC and APCKLFFWKTFSHC) as queries against the $S$. canicula genome draft assembly (version 1 , unpublished). S. canicula full-length SSTd cDNA (but not SSTe cDNA) was also retrieved from $S$. canicula Illumina transcriptome (version 1, unpublished).

The SST repertoire of $S S T$ genes in $R$. typus and $C$. milii was sought by TBLASTN using the $S$. canicula SST cDNA sequences as queries against the $R$. typus and $C$. milii genome assembly databases (Read et al. 2017; Venkatesh et al. 2014).

Protein sequences were predicted by using consensus splice donor and acceptor site and by sequence identity comparison with related SST genes. The signal peptide cleavage site was determined using SignalP (http://www.cbs.dtu.dk/services/SignalP/) (Petersen et al. 2011).

\section{Molecular cloning of the coding sequence of $S$. canicula SSTe cDNA}

5'RACE-ready cDNAs used for this procedure were constructed as previously described (Gaillard et al. 2018). The coding sequence of the $S$. canicula SSTe cDNA was amplified by nested PCR using the Advantage 2 PCR kit (Clontech). Nested PCR was carried out in a MyCycler thermal cycler (Bio-Rad, Marne la Coquette, France) with the following primers, csSSTe Rev x Universal Primer A Mix (UPM) then csSSTe Rev Nest x Nested Universal Primer A Mix (NUP) (see Table S1) using the manufacturer's procedure. PCR amplifications were carried out for 35 cycles (denaturation $94^{\circ} \mathrm{C}$, $30 \mathrm{sec}$; annealing between 57 and $60^{\circ} \mathrm{C}$ depending on the $\mathrm{Tm}$ of the primers, $30 \mathrm{sec}$; and extension $72^{\circ} \mathrm{C}, 30 \mathrm{sec}$ ) and a final extension 
of $72^{\circ} \mathrm{C}$ for $7 \mathrm{~min}$. The product of the nested PCR was subcloned into the pGEM-T vector (Promega, Charbonnières-les-Bains, France) and sequenced (Value Read Sequencing at MWG Biotech, Ebersberg, Germany).

\section{Synteny analysis}

To generate synteny maps, genes flanking $C$. milii and $R$. typus $S S T$ genes were obtained from the NCBI database. Non-protein coding genes and genes coding unknown proteins were excluded from further analysis. Note that $S S T b$ was not included in the study since it is absent in both $C$. milii and $R$. typus, while in $S$. canicula, it is located on a very short scaffold that did not contain any other genes. Genes flanking the SST genes in eight additional osteichthyan species, namely human, chicken (G. gallus), green anole lizard (A. carolinensis), Western clawed frog (X. tropicalis), coelacanth (L. chalumnae), spotted gar (L. oculatus), zebrafish (Danio rerio) and medaka (Oryzias latipes), were obtained from Genomicus (version 92.01) (Muffato et al. 2010).

\section{Phylogeny analysis}

A set of 147 vertebrate SST precursor sequences was primarily collected from Yun et al. (2015) and Tostivint et al. (2016) and supplemented by the chondrichthyan sequences characterized in the present study. All these sequences were aligned using the Muscle algorithm (Edgar 2004) then manually adjusted. The phylogeny of SST precursors was inferred by Maximum Likelihood using PhyML (Guindon \& Gascuel 2003) with the LG model of substitution combined to the neighbor-joining interchange (NNI) method on Seaview browser (Gouy et al. 2010). The robustness of the tree was assessed by the approximate likelihood test (aLRT) SHlike branch support and by bootstrapping procedure from 500 data set replicates. The Genbank accession numbers for all sequences used in the analysis are listed in Figure S1. The alignment is presented in Figure S2.

Tissue expression of $S$. canicula SSTd and SSTe genes by reverse transcriptase-polymerase chain reaction (RT-PCR) amplification

The expression profiles of $S$. canicula SSTd and SSTe genes were examined by RT-PCR, as previously described (Quan et al. 2013, Gaillard et al. 2018). Total RNA was extracted from various tissues, including telencephalon, diencephalon, mesencephalon, cerebellum, brain stem, spinal cord, skeletal muscles, heart, spleen, gills, stomach, duodenum, valvular intestine, pancreas, liver, kidney, ovary and testis and purified by using RNAeasy Plus Mini kit (Qiagen, Courtabœuf, France). For each tissue, $\sim 330$ ng of total RNA were reverse transcribed using ImProm-II Reverse Transcription System (Promega, Charbonnières, France). Gene-specific primers of S. canicula SSTd and SSTe were designed according to the predicted sequences (Table S1). PCR amplifications were carried out for 35 cycles (denaturation $94^{\circ} \mathrm{C}, 30 \mathrm{sec}$; annealing between 57 and $60^{\circ} \mathrm{C}$ depending on the Tm of the primers, $30 \mathrm{sec}$; and extension $72^{\circ} \mathrm{C}, 30 \mathrm{sec}$ ) and a final extension of $72^{\circ} \mathrm{C}$ for $7 \mathrm{~min}$. The $S$. canicula Egfl gene was amplified in parallel with specific primers (Table S1) to verify the quality and quantity of all cDNAs samples. Negative controls were performed without cDNA template. All PCR products were electrophoresed through $2.0 \%$ agarose gel and stained with ethidium bromide and then detected under UV light with the ChemiDoc Touch Imaging System (BioRad). Three independent PCR amplifications were performed to check the consistency of amplification.

\section{Results}

Structure of $S$. canicula SSTd (SST2) and SSTe (SST3) precursor cDNAs and genes 
The nucleotide and deduced amino acid sequences of S. canicula SSTs including SSTd and SSTe are shown in Figure S3. The coding region of the SSTd cDNA consists of 315 nucleotides (nt) that encode a 105-amino acid (aa) protein (PSSTd ) including a 20-residue potential signal sequence (Petersen et al. 2011). The primary structure of PSSTd contains a monobasic processing site at position 91 (Lys) potentially yielding the mature 14-aa peptide SSTd (SST2), TPCKLFFWKTFSHC, plus one dibasic and one monobasic processing sites at positions 87-88 (Arg-Arg) and 79 (Arg) respectively, potentially yielding 17- and 26-aa NH2-extended forms of SSTd.

The coding region of the SSTe cDNA consists of 321 nt that encode a 106-aa protein with a 18residue potential signal sequence. The SSTe sequence is flanked by a pair of basic residues (Arg-Lys) at position 92-93 potentially yielding the mature 14-aa peptide SSTe (SST3), NCKNFFWKTYTLC, plus two monobasic processing sites at positions 85 (Arg) and 90 (Arg) respectively, potentially yielding 17- and 22-aa NH2-extended forms of SSTe.

Comparison of the cDNAs with genomic sequences revealed that SSTd and SSTe genes are each composed of two exons and one intron (Figure S4). For each gene, exon 1 encodes the 5' UTR, the signal peptide and the $\mathrm{N}$-terminus of the prepro-peptide while exon 2 encodes the $\mathrm{C}$ terminus of the precursor, including the putative mature peptide.

\section{Repertoire of SST cDNAs and/or genes in cartilaginous fish}

The entire repertoire of $S S T$ cDNAs and/or genes found from $S$. canicula, $R$. typus and $C$. milii databases is shown in Figures S3-S8. S. canicula SST family appears as the richest with no less than five SST genes: the three genes previously reported in Quan et al. (2013), namely SSTa, $S S T b$ and SSTc, plus two additional genes characterized in the present study, SSTd and SSTe. All these genes, with the exception of $S S T b$, were retrieved from $R$. typus and $C$. milii. Only a partial sequence of the SSTd gene could be detected from L. erinacea (already reported in Liu et al. 2010).

\section{General organization of the chondrichthyan SST genes and SST precursors}

The molecular organization of the SST precursors is the same in all species examined, with a signal peptide followed by a long central segment, a conserved pair of basic residues and, finally, the SST sequence at their C-terminus (Figure 1). All putative SST possess a cyclic conformation ensured by the presence of two Cys residues, and their cyclic region encompasses the Phe-Trp-Lys motif. The structure of the corresponding genes is also very well conserved with two exons and one intron (Figures S4, S5, S7). Note that the first exon of S. canicula SSTd and that of S. canicula SSTa (SST1) exhibit almost the same nucleotide sequence (Figure S4). This sequence identity was also found in the corresponding cDNA identified by RT-PCR ruling out an artefact of genome assembly (Figure S3).

\section{Structural features of the chondrichthyan putative SST tetradecapeptides}

- SSTa (SST1)

The primary structure of SSTa is AGCKNFFWKTFTSC both in S. canicula and $R$. typus. In $C$. milii, it exhibits one substitution, Ser vs Asn, at position 5, as previously reported in the ratfish H. colliei (Conlon 1990) (Table S2). It is to note that the genomic SSTa sequence encodes for a Leu in place of Phe at position 6 that was not retrieved in the cDNAs (Figures S3 and S4). Chondrichthyan prepro-SSTa exhibit high sequence identity (ranging from 66.3 to $74.1 \%$ between one another) (Table S3).

- SSTb (SST5)

As mentioned above, the SSTb sequence could only be found in S. canicula (see Quan 2013 for a complete description of the precursor). Just as a reminder, $S$. canicula SSTb exhibits the same primary structure as SSTa (SST1) (Table S2). 
- SSTc (SST6)

SSTc exhibits two substitutions at positions 1 and 2 when compared to SSTa (Table S2). The residue at position 2 is a Pro, except in $R$. typus. The residue at position 1 is more variable. Chondrichthyan prepro-SSTc exhibit high sequence identity (ranging from 53.8 to $86.7 \%$ between one another) (Table S3).

- SSTd (SST2)

When compared to that of SSTa, the primary structure of SSTd can be distinguished by the SerHis-Cys (SHC) motif at its C-terminus (Table S2). Variable residues are present at position 1 and 2. However, the residue at position 2 is generally a Pro, except in C. milii. Chondrichthyan prepro-SSTd exhibit high sequence identity (ranging from 51.9 to $76 \%$ between one another) (Table S3).

- SSTe (SST3)

In all species examined, the primary structure of SSTe exhibits the unique Tyr-Thr-Leu-Cys (YTLC) motif at its C-terminus (Table S2). Only the residue at position 1 is different depending on the species. S. canicula and C. milii prepro-SSTe exhibit only $38.1 \%$ identity (Table S3).

\section{Synteny analysis of $S S T$ genomic regions}

In both $R$. typus and C. milii, SSTe (SST3) and SSTa (SST1) are tandemly arranged on the same scaffold (Figure 2, Table S4). However, this scaffold is very short and does not contain any other genes. On the basis of our previous results suggesting that SSTa probably corresponds to SST1 (Quan et al. 2013), we aligned the chondrichthyan SSTa-SSTe scaffold with the SST1 locus from the other species analyzed. In teleosts, SST3 is localized in tandem with SST1, as SSTe and SSTa in cartilaginous fish.

In spotted gar, the SST1-SST3 locus bears a third SST gene, here called SST4, surrounded by Nyap2 and Irs1 (Figure 2, Table S4). In teleosts, the SST known as SST4 is located on a different chromosome than that bearing SST1 and SST3. Its closest neighbors are Nyap $2 b$ and Fgf12a (Figure 2, Table S4). The co-orthologs of these two latter genes, Nyap $2 a$ and Fgf12b, are present in the vicinity of both SST1 and SST3. Thus, the teleost SST4 can be view as either an ortholog to gar SST4 (as this is what its name implies) or a 3R duplicate of either SST1 or SST3. These three possibilities are illustrated in Figure 2.

$R$. typus and C. milii SSTd (named SST2 in Figure 3) were found in a genomic region containing several genes, Kiflb, Pgb, Cenps and Dffa, that are also present in the vicinity of the human SST2 gene (Figure 3, Table S4). It is to note that the gar and zebrafish SST genes present in the syntenic region correspond to genes previously known as SST6.

The neighborhood of the R. typus and C. milii SSTc (named SST6 in Figure 3) is composed of several genes, Tardbp, Masp2 and Srm, that, in the other species examined, were present in the vicinity of $S S T$ genes previously known as SST2, notably in chicken, spotted gar, zebrafish and medaka (Figure 3, Table S4).

\section{Phylogeny analysis of SST precursors}

Figure 4 displays an unrooted tree constructed from 147 selected vertebrates SST precursor sequences using maximum likehood phylogeny analysis. In the resulting tree, osteichthyan SST sequences cluster into six main clades, SST1-SST6. Bootstrap support values for these six groups were 53,10,94, 29, 68 and $75 \%$, respectively. The only discrepancy of this patterning is the presence of the gar SST4 sequence into the SST3 clade. It is to note that the actinopteygian SST sequences belonging to the SST2 clade were previously known as SST6. Likewise, chicken, lungfish, sturgeon and teleost SST sequences included in the SST6 clade were previously known as SST2.

Chondrichthyan SSTb, SSTc and SSTd branch at the base of the SST5, SST6 and SST2 clades, respectively. Gnathotostome SST2-SSTd and SST6-SSTc appeared as two sister groups as well 
as osteichthyan SST1 and SST3. For their part, chondrichthyan SSTa and SSTe formed a monophyletic group that branch at the base of the osteichthyan SST1-SST3 clade.

As previously reported (Tostivint et al. 2016), lamprey SST sequences did not group to any of the gnathostome SST clades.

\section{Tissue-specific expression of $S$. canicula SSTd (SST2) and SSTe (SST3) genes}

The distribution of S. canicula SSTd and SSTe mRNAs in various tissues was examined by RTPCR. Figure 5 shows the results of one representative experiment out of three performed with identical results. SSTd mRNA was detected primarily in the brain (notably in the diencephalon, mesencephalon and brainstem) and spinal cord. In contrast, SSTe mRNA appeared particularly abundant in the central nervous system (in all subdivisions examined except in the cerebellum) as well as in the intestinal tract and pancreas. Note that traces of SSTd mRNA were also seen in the spleen, gills, pancreas and gonads.

\section{Discussion}

The main aim of the present study was to determine the repertoire of the SST gene family in cartilaginous fish. For this purpose, the genome and/or transcriptome databases from three representative chondrichthyan species were examined, namely the small-spotted catshark $S$. canicula (unpublished, see Gaillard et al. 2018 for more details), the whale shark, $R$. typus (Read et al. 2017) and the elephant shark C. milii (Venkatesh et al. 2014). In S. canicula, two new SST genes, named SSTd and STTe were found, in addition to the three SST genes, SSTa, $S S T b$ and SSTc, previoulsly identified (Quan et al. 2013). All these genes, with the exception of $S S T b$, were retrieved from $R$. typus and $C$. milii. It is noteworthy that the occurrence of partial sequences of the SSTc (Tostivint et al. 2008) and SSTd genes (Liu et al. 2010) were already reported in both $L$. erinacea and/or C. milii. In contrast, SSTe appeared as a totally novel gene. The nomenclature used above (SSTa-SSTe) was initially (and provisionally) proposed to designate the $S$. canicula SSTs without prejudice to their evolutionary relationships with other vertebrate SSTs (Quan et al. 2013). Synteny comparisons and phylogeny analysis were subsequently carried out in order to reveal the phylogenetic status of all these genes.

Synteny comparisons suggest that SSTd is the chondrichthyan counterpart of SST2 since both S. canicula and C. milii SSTd reside in the same chromosomal environment as that of human SST2. For the same reasons, SSTc can be legitimately viewed as the chondrichthyan counterpart of SST6. These conclusions are quite consistent with our phylogeny analysis because SSTd and SSTc precursor sequences branch at the base of the SST2 and SST6 clades, respectively, although the bootstrap support values of the new clades are extremely weak (4\% and $37 \%$ respectively).

Our investigations on the chondrichtyan SSTC and SSTe also led us to redefine the relationships between SST2 and SST6. The SST2 gene was been first characterized in frog (Tostivint et al. 1996) and mammals (de Lecea et al. 1996, 1997a), then in most other vertebrates, including chicken (Trabucchi et al. 2003), lungfish (Trabucchi et al. 1999) and sturgeons (Trabucchi et al. 2002, Li et al. 2009) (see Tostivint et al. 2008 for review). All peptides encoded by SST2 possessed a Pro residue at position 2 and most of them exhibited the APCKNNFWKTFTSC sequence (Quan et al. 2013), except in mammals and some other species such as frog (Vaudry et al. 1992; Tostivint et al. 2008 for review) (Table S2). As for, the SST6 gene was initially characterized in only one teleost species, the zebrafish (Liu 2010), then in spotted gar (Tostivint et al. 2013) and coelacanth (Yun et al. 2015) (Table S2). In the present study, we found an ortholog of this gene in several other teleost species belonging to various groups (particularly from otocephala) but not from euteleosts. Peptides encoded by SST6 also shared a Pro at position 2, suggesting a common origin with SST2. In support of this latter view, in both zebrafish and gar, SST2 and SST6 are closely linked on the same chromosome, indicating that 
they most likely arose by tandem duplication. Our phylogeny and synteny analyses showed that most of the non-mammalian SST sequences previously referred as to SST2 are not orthologous to cortistatin (the other name of the mammalian SST2) but actually correspond to SST6. Such is notably the case for birds (chicken) and fish (lungfish, sturgeons, bony and cartilaginous fish), but not for reptiles (lizard) and amphibians (marsh frog). Conversely, all SST sequences previously referred to as SST6 appeared to correspond to SST2. As a result, all the SST genes concerned with these observations were renamed accordingly. It is to note that the nomenclature adopted in the present article was already proposed in Yun et al. (2015) but without explicit justification.

In $R$. typus and $C$. milii, SSTe and SSTa are tandemly arranged suggesting that both genes arose by local duplication of a common ancestral gene. Synteny analysis of the SSTa-SSTe locus was inhibited by the fact that SSTa and SSTe were the only genes present on their scaffold. However, based on previous results showing that SSTa probably corresponds to SST1 (Quan et al. 2013), the most credible assumption is that SSTe corresponds to SST3 because in all species possessing both SST1 and SST3, these genes are systematically localized in tandem (Tostivint et al. 2004, 2008, 2013, 2014). Phylogeny analysis is in agreement with close evolutionary relationships between SSTa and SSTe, on the one hand, and SST1 and SST3, on the other hand. However, it does not fully support the orthology between SST1 and SSTa, on one hand, and SST3 and SSTe, on the other hand, since chondrichthyan SSTa and SSTe sequences did not strictly group with osteichthyan SST1 and SST3, respectively. One explanation to reconcile these contradictory results would be that SST1/SSTa and SST3/SSTe genes were subjected to concerted evolution, a genetic process that leads to homogenize repetitive DNA sequences over time (Liao 1999). Indeed, the major hallmark of concerted evolution is that paralogous genes within species are more similar to one another than they are to their orthologous counterparts in closely related species (Liao 1999). DNA recombination mechanisms, especially unequal crossover and gene conversion, are responsible for concerted evolution (Liao 1999). Interestingly, we have shown that nucleotide sequences of the first exon of S. canicula SSTa and S. canicula SSTe are almost identical (93.5\% of identity), while those of the second exon are much more divergent (only $61 \%$ of identity). Such level of similarity may be a good indicator of a recent recombination event occurring between the two genes.

The phylogenetic status of the S. canicula SSTd and SSTe genes suggested by synteny and phylogeny analyses is consistent with their tissue expression pattern since $S S T d$, as $S S T 2$, is primarily expressed in the central nervous system (SNC) (de Lecea et al. 1997b, Lin et al. 1999, Trabucchi et al. 1999, 2003), while SSTe, as SST3, is expressed both in the SNC and the gastrointestinal tract (Moore et al. 1995, Xing et al. 2005). Further studies at the cellular level will be needed to speculate about the functions of the corresponding peptides.

In contrast to the other SST genes found in cartilaginous fish, SSTb was detected only in $S$. canicula and not in R. typus and C. milii. Phylogeny analysis showed that SSTb grouped into the SST5 clade, as previously reported (Quan et al. 2013). However, the orthology relationships between $S S T b$ and osteichthyan SST5 could not be confirmed by synteny analysis since the $S$. canicula SSTb-bearing scaffold was too short.

Among the six SST paralogs characterized so far in gnathostomes, SST4 is the only one not to exist in cartilaginous fish. Up to now, the SST4 gene was considered as a SST1 paralog generated by 3R (Liu et al. 2010), in agreement with the fact that it was found only in teleosts (Figure S9A). Recently, however, Yun et al. (2015) reported the occurrence of a so-called SST4 ortholog in spotted gar. This finding is clearly in conflict with the Liu's view since the gar lineage is known to have diverged from teleosts before the 3R (Figure S9B) (Braasch et al. 2016). In the present study, synteny analysis showed that teleost SST4 can be equally viewed as orthologous to the gar SST4, or co-orthologous to either teleost SST1 or teleost SST3, without 
the possibility to decide between these three conceptions. Indeed, in both zebrafish and medaka, the SST4-bearing chromosome block contains only very few paralogs of the neighboring genes of SST1 and SST3 (such as Fgf12 and Nyap2), making it impossible to precisely place the teleost SST4 in relation to gar SSTs. Examination of other teleost species whose genome is available in Ensembl failed to resolve the problem (data not shown). What is clear, however, is that phylogeny analysis absolutely does not support the orthology of gar SST4 and teleost SST4 since gar SST4 groups within the gnathostome SST3 clade, thus without any obvious relationships with the teleost SST4 clade. Rather, the very high level of sequence similarity between gar SST3 and gar SST4 (91 \% nucleotide identity) strongly suggests that gar SST4 arose very recently by tandem duplication of SST3. It therefore appears unlikely that gar SST4 and teleost SST4 are orthologous unless gar SST4 and SST3 were subjected to concerted evolution, as shown above for $S$. canicula SST1/SSTa and SST3/SSTe.

\section{Concluding remarks}

Over the past few years, significant advances have been made in the understanding of the evolution of the SST family. One of the most important results has been the demonstration that the current diversity of the family was initially caused by 2R (Liu et al. 2010, Tostivint et al. 2013, 2014). According to this view, the last common ancestor of vertebrates possessed four SST genes, three of which, SST1, SST2 and SST5, are still present in all extant vertebrate species. A fourth $S S T$ gene (referred to as SST? in Figure 6) is likely to have been generated by $2 \mathrm{R}$ but since this gene has never been found in extant vertebrate species, it is assumed to have been lost very early after $2 \mathrm{R}$. Conversely, the rest of the diversity of the family, represented by SST3, SST4 and SST6, was considered restricted to only some groups of bony fish (Tostivint et al. 2013, 2014): actinopterygians for SST3 and SST6 and teleosts for SST4.

The principal result of the present study is the demonstration of an unexpected diversity of SST genes in cartilaginous fish. Thus, in $S$. canicula, not less than five SST genes, SST1, SST2, SST3, SST5 and SST6, have been identified, while only three of them were characterized so far (Quan et al. 2013). Therefore, contrary to what was previously though, it is likely that all these five genes were already present in the gnathostome ancestor (Figure 6).

It is to note that our study did not provide any new data supporting the impact of $2 \mathrm{R}$ in the evolution of the SST gene family due to the small size of the scaffolds from the three chondrichthyan species examined. Thus, within the regions containing SST genes, Masp 1 and Masp 2 were the only genes indicating that SST1 and SST3, on the one hand and SST2 and SST6, on the other hand, belong to the same paralogon. In contrast, our study confirm the idea that SST3 and SST6 likely arose by tandem duplications from SST1 and SST2, respectively (Figure 6). In strict logic, however, we must admit that it is currently impossible to know which of SST1 and SST3, on the one hand and SST2 and SST6, on the other hand are the ancestral genes. The uncertainty on this matter will remain as long as the phylogenetic status of lamprey SSTs is unsolved (Tostivint et al. 2016) because the lamprey SST sequences are the only ones that could be currently used as outgroups. Thus, in Figure 6, SST1 and SST2 are displayed as ancestral genes in a totally arbitrary manner.

Another important result of the present study was the finding that SST6 was not totally lost in sarcopterygians, as previously thought, because it is still present in coelacanth and birds (Figure 6). In this context, it is noteworthy that chicken SST6 was initially but mistakenly reported as SST2 (Trabucchi et al. 2002), now refuted by synteny and phylogeny analysis. In a general way and for the same reasons, all teleost genes previously known as SST6 had to be renamed SST2, and vice versa. Notably, the true SST2 is also present in amphibians (but not in Xenopus), sauropsids (but not in birds) and mammals (Figure 6). Taken together, our result show that SST2 and SST6 have been lost independently and repeatedly during evolution: in euteleosts, birds and some amphibians for SST2 and amphibians and mammals for SST6 (Figure 6). After 
their appearance, SST3 and SST5 were also secondarily lost in sarcoperygians and tetrapods, respectively.

Finally, the present work was unable to precisely determine the phylogenetic status of teleost SST4. In particular, whether teleost SST4 and gar SST4 are truly orthologous or not remains to be investigated. Further studies in other species than those examined here will be needed to address this issue. 


\section{Acknowledgements}

This work has been supported by the Centre National de la Recherche Scientifique (CNRS), the Muséum National d'Histoire Naturelle (MNHN) and the Agence Nationale de la Recherche (ANR) grant NEMO ( $\mathrm{n}^{\circ}$ ANR-14-CE02-0020-01 HT). The authors thank Hélène Mayeur (Observatoire Océanologique de Banyuls, France), and Patrick Wincker, Jean-Marc Aury and the Genoscope staff (Evry, France) for catshark genome sequencing and assembly. They also thank Dr Nicolas Buisine for helpful discussions. Finally, they are very grateful to Mitchell Fleming and Stephan Couderq (Museum National d'Histoire Naturelle) for the English corrections. 


\section{Legends to figures}

Figure 1. Alignment of the amino acid sequences of prepro-SST1 (PSSTa), -SST2 (PSSTd), SST3 (PSSTd) and -SST6 (PSSTc) characterized from cartilaginous fish. (*) denotes conserved residues. SST peptide sequences are in red. Potential cleavage sites are in bold. Signal peptides are in grey. (p), partial sequences. Sequences of S. canicula SST2 (SSTd) and SST3 (SSTe) cDNAs have been deposited in the GenBank database under the accession numbers MK491629 and MK491630, respectively.

Figure 2. Synteny of genes in the SST1-SST3 locus in R. typus and C. milii plus eight selected bony vertebrate species (human, chicken, anole lizard, western clawed frog, coelacanth, spotted gar, zebrafish and medaka). Genes are represented by block arrows. Genes with conserved synteny are coloured. For the teleost SST4 gene, three possible positions are proposed with respect to the gar SSTs (see the text for more details). Position of the genes (in megabases $-\mathrm{Mb}$ ) is displayed below each box, according to the Ensembl database. Empty circles indicate the end of scaffolds. Letters inside the boxes refer to the specific and provisional nomenclature of $S$. canicula SSTs initially proposed in Quan (2013). The detailed chromosomal locations of genes displayed in this map are included in Table $\mathbf{S 4}$.

Figure 3. Synteny of genes in the SST2-SST6 locus in R. typus and C. milii plus eight selected bony vertebrate species (human, chicken, anole lizard, western clawed frog, coelacanth, spotted gar, zebrafish and medaka). Letters inside the boxes refer to the nomenclature of $S$. canicula SSTs proposed in Quan (2013), while numerals refer to the previous nomenclature of vertebrate SST genes (Tostivint 2013, 2014). Legends are the same as in the Figure 3.

Figure 4. Phylogenetic tree of vertebrate SST precursor sequences. Phylogenetic analysis of 147 vertebrate SST amino acid sequences was performed using Maximal Likelihood, with 500 bootstrap replicates. The number shown at each branch node indicates in percentage the bootstrap value. Sequence references and alignment are given in Figure S1 and Figure S2, respectively

Figure 5. Tissue distribution of S. canicula SST2 (SSTd) and SST3 (SSTe) mRNAs using RTPCR. Parallel amplification of dogfish Egfl mRNA served as internal control. NC, nontemplate control.

Figure 6. A proposed evolutionary scheme for the evolution of the $S S T$ gene family in gnathostomes with special emphasis on chondrichthyan species (in red). The names of the different paralogous genes are given in the boxes. Note the unresolved status of the teleost SST4. Gar SST4 is shown here to have arisen independently of teleost SST4. Boxes connected by a vertical line represent linked genes. Crossed-out boxes represent lost genes. Double-headed arrows represent local duplications. R, rounds of whole genome duplication. 


\section{Supplementary data}

Table S1. Sequences of the oligonucleotides used for PCR amplifications.

Table S2. Primary structure of SST-related peptides from different vertebrate species. Peptides from chondrichthyan species are in red. Conserved residues are in bold.

Table S3. Pair-wise comparison of full-length SST protein sequences from S. canicula, $R$. typus, L. erinacea and C. milii. Values represent percentage of amino acid identity.

Table S4. Chromosomal locations of gene orthologs in the synteny analysis of the SST loci. All human gene names in the table are approved HGNC symbols and the genes from other species have been given the name of their human orthologs. Chromosomal locations of genes are expressed in bp. Blanks represent genes that have not been found in the current genome databases. Non-syntenic genes are in grey.

Figure S1. Protein sequences of prepro-SSTs used for phylogenetic analysis and their accession numbers. Chondrichthyan sequences are in red.

Figure S2. Amino acid sequence alignment of the SST family used for the phylogenetic analysis. The alignment was made using Muscle and then manually optimized. Conserved residues between all orthologs are in black bold. Characteristic residues of each ortholog are colored.

Figure S3. Nucleotide sequence and deduced amino acid sequence of S. canicula cDNAs. Nucleotides are numbered 5' to 3 ' and amino acids $\mathrm{N}$-terminal to C-terminal from the putative starting methionine. Signal peptides are in grey. SST peptide sequences are in red. Potential cleavage sites are in bold. Polyadenylylation signals, when detected, are underlined.

Nucleotide sequence (almost) identical between SSTI and SST3 is in red.

Figure S4. Sequence of S. canicula SST genes, inferred from the cDNA sequences given in Figure S3. Exons are indicated by colored boxed. The exon/intron boundary consensus sequences (GT/AG) are highlighted in red. Initiation (ATG) and stop codons are boxed. Polyadenylation signals are underlined. The identity of the scaffolds containing the sequences is indicated. Nucleotide sequence (almost) identical between SST1 and SST3 is in red.

Figure S5. Predicted sequence of $R$. typus SST genes, inferred from the SST cDNA sequences given in Figure S6. Legends are the same as in the Figure S4.

Figure S6. Nucleotide and deduced amino acid sequences of $R$. typus SST cDNAs. Legends are the same as in the Figure $\mathbf{S 3}$.

Figure S7. Predicted sequence of C. milii SST genes, inferred from the $S S T$ cDNA sequences given in Figure S8. Legends are the same as in the Figure S4.

Figure S8. Nucleotide and deduced amino acid sequences of $C$. milii SST cDNAs. Legends are the same as in the Figure S3.

Figure S9. Two alternative conceptions regarding the phylogenetic status of the teleost SST4 gene. A. Conception from Liu et al., (2010). B. Conception from Yun et al. (2015). 


\section{References}

Altschul, S.F., Gish, W., Miller, W., Myers, E.W., Lipman, D.J., 1990. Basic local alignment search tool. J. Mol. Biol. 215, 403-410.

Brazeau, P., Vale, W., Burgus, R., Ling, N., Butcher, M., Rivier, J., Guillemin. R., 1973. Hypothalamic polypeptide that inhibits the secretion of immunoreactive pituitary growth hormone. Science 179, 77-79.

Conlon, J.M., Agoston, D.V., Thim, L., 1985. An elasmobranchian somatostatin: primary structure and tissue distribution in Torpedo marmorata. Gen. Comp. Endocrinol. 60, 406413.

Conlon, J.M., Askensten, U., Falkmer, S., Thim, L., 1988. Primary structures of somatostatins from the islet organ of the hagfish suggest an anomalous pathway of posttranslational processing of prosomatostatin-1. Endocrinology. 122:1855-9.

Conlon, J.M., 1990. [Ser ${ }^{5}$ ]-somatostatin-14: isolation from the pancreas of a holocephalan fish, the Pacific ratfish (Hydrolagus colliei). Gen. Comp. Endocrinol. 80, 314-320.

Dehal, P., Boore, J.L., 2005. Two rounds of whole genome duplication in the ancestral vertebrate, PLoS Biol. 3, e314.

de Lecea, L., Criado, J.R., Prospero-Garcia, O., Gautvik, K.M., Schweitzer, P., Danielson, P.E., Dunlop, C.L., Siggins, G.R., Henriksen, S.J., Sutcliffe, J.G., 1996. A cortical neuropeptide with neuronal depressant and sleep-modulating properties. Nature 381, 242245.

de Lecea, L., Ruiz-Lozano, P., Danielson, P.E., Peelle-Kirley, J., Foye, P.E., Frankel, W.N., Sutcliffe, J.G. 1997a. Cloning, mRNA expression, and chromosomal mapping of mouse and human preprocortistatin. Genomics 42, 499-506.

de Lecea, L., del Rio, J.A., Criado, J.R., Alcántara, S., Morales, M., Danielson, P.E., Henriksen, S.J., Soriano, E., Sutcliffe, J.G., 1997b. Cortistatin is expressed in a distinct subset of cortical interneurons. J. Neurosci. 17, 5868-5880.

Edgar, R.C., 2004. MUSCLE: a multiple sequence alignment method with reduced time and space complexity. BMC Bioinformatics 5, 113.

Elphick, M.R., Mirabeau, O., Larhammar, D., 2018. Evolution of neuropeptide signalling systems. J. Exp. Biol. 221, jeb151092.

Gaillard, A.L., Tay, B.H., Perez Sirkin, D.I., Lafont, A.G., De Flori, C., Vissio, P.G., Mazan, S., Dufour, S., Venkatesh, B., Tostivint, H., 2018. Characterization of gonadotropinreleasing hormone $(\mathrm{GnRH})$ genes from cartilaginous fish: evolutionary perspectives. Front Neurosci. 12, 607.

Gouy, M., Guindon, S., Gascuel, O., 2010. SeaView version 4: A multiplatform graphical user interface for sequence alignment and phylogenetic tree building. Mol. Biol. Evol. 27, 221224.

Guindon, S., Gascuel, O., 2003. A simple, fast, and accurate algorithm to estimate large phylogenies by maximum likelihood. Syst. Biol. 52, 696-704.

Günther, T., Tulipano, G., Dournaud, P., Bousquet, C., Csaba, Z., Kreienkamp, H.J., Lupp, A., Korbonits, M., Castaño, J.P., Wester, H.J., Culler, M., Melmed, S., Schulz, S., 2018. International Union of Basic and Clinical Pharmacology. CV. Somatostatin Receptors: Structure, Function, Ligands, and New Nomenclature. Pharmacol. Rev. 70, 763-835.

Li, C.J., Wei, Q.W., Zhou, L., Cao, H., Zhang, Y., Gui, J.F., 2009. Molecular and expression characterization of two somatostatin genes in the Chinese sturgeon, Acipenser sinensis. Comp. Biochem. Physiol. A Mol. Integr. Physiol. 154, 127-134.

Liao, D., 1999. Concerted evolution: molecular mechanism and biological implications. Am. J. Hum. Genet. 64, 24-30.

Lin, X., Otto, C.J., Peter, R.E., 1999. Expression of three distinct somatostatin messenger ribonucleic acids (mRNAs) in goldfish brain: characterization of the complementary 
deoxyribonucleic acids, distribution and seasonal variation of the mRNAs, and action of a somatostatin-14 variant. Endocrinology 140, 2089-2099.

Liu, Y., Lu, D., Zhang, Y., Li, S., Liu, X., Lin, H., 2010. The evolution of somatostatin in vertebrates. Gene 463, 21-28.

Moore, C.A., Kittilson, J.D., Dahl, S.K., Sheridan, M.A., 1995. Isolation and characterization of a cDNA encoding for preprosomatostatin containing $\left[\mathrm{Tyr}^{7}, \mathrm{Gly}^{10}\right]$-somatostatin-14 from the endocrine pancreas of rainbow trout, Oncorhynchus mykiss. Gen. Comp. Endocrinol. 98, 253-261.

Muffato, M., Louis, A., Poisnel, C.E, Roest Crollius, H., 2010. Genomicus: a database and a browser to study gene synteny in modern and ancestral genomes. Bioinformatics 26, 11191121.

Ocampo Daza, D., Sundström, G., Bergqvist, C.A., Larhammar, D., 2012. The evolution of vertebrate somatostatin receptors and their gene regions involves extensive chromosomal rearrangements. BMC Evol. Biol. 12, 231.

Petersen, T.N., Brunak, S., von Heijne, G., Nielsen, H., 2011. SignalP 4.0: discriminating signal peptides from transmembrane regions. Nat. Methods 8, 785-786.

Putnam, N.H., Butts, T., Ferrier, D.E., Furlong, R.F., Hellsten, U., Kawashima, T., RobinsonRechavi, M., Shoguchi, E., Terry, A., Yu, J.K., Benito-Gutiérrez, E.L., Dubchak, I., GarciaFernàndez, J., Gibson-Brown, J.J., Grigoriev, I.V., Horton, A.C., de Jong, P.J., Jurka, J., Kapitonov, V.V., Kohara, Y., Kuroki, Y., Lindquist, E., Lucas, S., Osoegawa, K., Pennacchio, L.A., Salamov, A.A., Satou, Y., Sauka-Spengler, T., Schmutz, J., Shin-I, T., Toyoda, A., Bronner-Fraser, M., Fujiyama, A., Holland, L.Z., Holland, P.W., Satoh, N., Rokhsar, D.S., 2008. The amphioxus genome and the evolution of the chordate karyotype. Nature 453, 1064-1071.

Quan, F.B., Kenigfest, N.B., Mazan, S., Tostivint, H., 2013. Molecular cloning of the cDNAs encoding three somatostatin variants in the dogfish (Scylorhinus canicula). Gen. Comp. Endocrinol. 180, 1-6.

Read, T.D., Petit, R.A. $3^{\text {rd }}$, Joseph, S.J., Alam, M.T., Weil, M.R., Ahmad, M., Bhimani, R., Vuong, J.S., Haase, C.P., Webb, D.H., Tan, M., Dove, A.D.M., 2017. Draft sequencing and assembly of the genome of the world's largest fish, the whale shark: Rhincodon typus Smith 1828. BMC Genomics 18, 532.

Sacerdot, C., Louis, A., Bon, C., Roest Crollius, H., 2018 Chromosome evolution at the origin of the ancestral vertebrate genome. Genome Biol. 19, 166.

Tostivint, H., Lihrmann, I., Bucharles, C., Vieau, D., Coulouarn, Y., Fournier, A., Conlon, J.M., Vaudry, H., 1996. Occurrence of two somatostatin variants in the frog brain: characterization of the cDNAs, distribution of the mRNAs, and receptor-binding affinities of the peptides. Proc. Natl. Acad. Sci. 93, 12605-12610.

Tostivint, H., Joly, L., Lihrmann, I., Ekker, M., Vaudry, H., 2004. Chromosomal localization of three somatostatin genes in zebrafish. Evidence that the $\left[\right.$ Pro $\left.^{2}\right]$-somatostatin-14 isoform and cortistatin are encoded by orthologous genes. J. Mol. Endocrinol. 33, R1-R8.

Tostivint, H., Lihrmann, I., Vaudry, H., 2008. New insight into the molecular evolution of the somatostatin family. Mol Cell. Endocrinol. 286, 5-17.

Tostivint, H., Quan, F.B., Bougerol, M., Kenigfest, N.B., Lihrmann, I., 2013. Impact of gene/genome duplications on the evolution of the urotensin II and somatostatin families. Gen. Comp. Endocrinol. 188, 110-117.

Tostivint H., Ocampo Daza, D., Bergqvist, C.A., Quan, F.B., Bougerol, M., Lihrmann, I., Larhammar, D., 2014. Molecular evolution of GPCRs: Somatostatin/urotensin II receptors. J. Mol. Endocrinol. 52, T61-86 
Tostivint, H., Dettaï, A., Quan, F.B., Ravi, V., Tay, B.H., Rodicio, M.C., Mazan, S., Venkatesh, B., Kenigfest, N.B., 2016. Identification of three somatostatin genes in lampreys. Gen. Comp. Endocrinol. 237, 89-97.

Trabucchi, M., Tostivint, H., Lihrmann, I., Jégou, S., Vallarino, M., Vaudry, H., 1999. Molecular cloning of the cDNAs and distribution of the mRNAs encoding two somatostatin precursors in the African lungfish Protopterus annectens. J. Comp. Neurol. 410, 643-652.

Trabucchi, M., Tostivint, H., Lihrmann, I., Sollars, C., Vallarino, M., Dores, R.M., Vaudry, H., 2002. Polygenic expression of somatostatin in the sturgeon Acipenser transmontanus: Molecular cloning and distribution of the mRNAs encoding two somatostatin precursors. J. Comp. Neurol. 443, 332-345.

Trabucchi, M., Tostivint, H., Lihrmann, I., Blähser, S., Vallarino, M., Vaudry, H., 2003. Characterization of the cDNA encoding a somatostatin variant in the chicken brain: Comparison of the distribution of the two somatostatin precursor mRNAs. J. Comp. Neurol. 461, 441-451.

Vaudry, H., Chartrel, N., Conlon, J.M., 1992. Isolation of $\left[\mathrm{Pro}^{2}\right.$, Met $\left.{ }^{13}\right]$ somatostatin-14 and somatostatin-14 from the frog brain reveals the existence of a somatostatin gene family in a tetrapod. Biochem. Biophys. Res. Commun. 188, 477-482.

Venkatesh, B., Lee, A.P., Ravi, V., Maurya, A.K., Lian, M.M., Swann, J.B., Ohta ,Y., Flajnik, M.F., Sutoh, Y., Kasahara, M., Hoon, S., Gangu, V., Roy, S.W., Irimia, M., Korzh, V., Kondrychyn, I., Lim, Z.W., Tay, B.H., Tohari, S., Kong, K.W., Ho, S., Lorente-Galdos, B., Quilez, J., Marques-Bonet, T., Raney, B.J., Ingham, P.W., Tay, A., Hillier, L.W., Minx, P., Boehm, T., Wilson, R.K., Brenner, S., Warren, W.C., 2014. Elephant shark genome provides unique insights into gnathostome evolution. Nature 505, 174-179.

Yun, S., Furlong, M., Sim, M., Cho, M., Park, S., Cho, E.B., Reyes-Alcaraz, A., Hwang, J.I., Kim, J., Seong, J.Y., 2015. Prevertebrate Local Gene Duplication Facilitated Expansion of the Neuropeptide GPCR Superfamily. Mol. Biol. Evol. 32, 2803-2817.

Xing, Y., Wensheng, L., Haoran, L., 2005. Polygenic expression of somatostatin in orangespotted grouper (Epinephelus coioides): molecular cloning and distribution of the mRNAs encoding three somatostatin precursors. Mol. Cell. Endocrinol. 241, 62-72. 


\section{Figure 1}

\author{
PSST1 (PSSTa) \\ C. milii \\ S. canicula \\ R. typus \\ C. milii \\ S. canicula \\ R. typus
}

\section{PSST2 (PSSTd)}

C. milii

L. erinacea (p)

S. canicula

R. typus

C. milii

L. erinacea (p)

S. canicula

R. typus

\section{PSST3 (PSSTe)}

C. milii

S. canicula

R. typus

C. milii

S. canicula

R. typus

PSST6 (PSSTC)

C. milii

S. canicula

R. typus

C. milii

S. canicula

R. typus
MSCGRVQCALALLSIALTVLSVTSAPAHDRYREILQRSLAAAGARSKPELTKYSLAQLLA MWCSRLQLSLALLS IALAVLSVSSAPTDNRYRE I LQRAMAATGSGGKAELTKYSLAQLLS

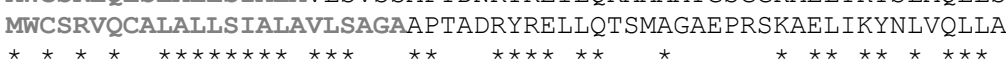

ELANAENEALEAEDMARATAQDEVRVELERSANPNLAQRERKAGCKSFFWKTFTSC ELENAENEALETDAM---GGRNEVRLELERS INPNLANRERKAGCKNFFWKTFTSC ELANAENEVLDGEDLPRMAKQDEVRVDLERSANPNSPQRERKAGCKNFFWKTFTSC

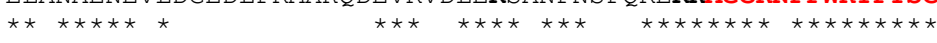

MQWQAFASVVCLLFIVTVQGVEPQEERMGLQLNREMVKDKKRLIAKLLAGLLDVDDNLLE -------------------MEDRT---TLQELTKNRKSLILKLLAGLLDVDDHLGE MKWLMTVSLMSLLYLLRVEGTDLLEERMKLOVNREMTKSRKNLIVKLLAGLLETDDNLLE ----MTVCLMSLLLLLSVEAADPLEERMKLQVNREVTKSRKYLMLKLLSGLLDPDDNLLE

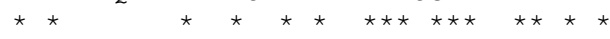

SVIAPLGLSKEEGTDFEERSAAGEIPPRKPKAGCKLFFWKTFSHC SGYTYSNTEEAGERSLEERAVVAVI PRREQKAPCKLFFWKTFSHC NGFAPLKPEEAEGTLFEERSVNAGIPRREQKTPCKLFFWKTFSHC NDFTSLSPGEEES-SLEERSVNAGIPRRPQKAPCKLFFWKTFSHC

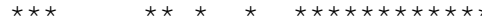

-MQARCTLTLAL--LSLAALGLSALPTHLRDQLVLQGSPAMELRDGVVRELIQAVADAES MWCSRLOLSLALLS IALAVLSVS SAPTDNRHRE I LORALDOELLKDTMMKLLPLVTDVDT $-------------------------------------D F L K D T I$ IQLLHVVADADK

KPQSEEAAEDF-APDEKAE---LRLALAPRWRKDCKNFFWKTYTLC EAGTLDVSNAETSEENTTPMKFQHRQLGMRMRKNCKNFFWKTYTLC DAVEVNASQVETPPXNKI PMKFQQRQLGTRMRKNCKNFFWKTYTLC

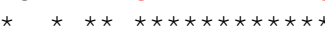

MOIL--VALISTIFLVSGVSTTTASLDDRFNLOSSREMNOERKEMILKLLSGLLDNAAVS MQLLGTASLLSMLLIV-SSVRATAPLEDRLSLRANQELNKERKEVILKLLSGLLDSS - - S MQLLGSASXVSILLIV-SSVKATAPLEDRLSLRANQELNKERKEVVLKLLSALLDSS - -S

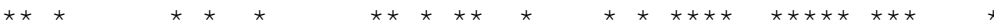

GQTGSEAGYPEPADGGPVMLEERSRYSQLPQRSRKVPCKNFFWKTFTSC NQIAIENTFPDPEDVEEMKVEERSRFSQLPQRERKAPCKNFFWKTFTSC NQMGIENTFPDQEDMEEMKVEDRSRFSQLPQRERKTSCKNFFWKTFTSC 
Human (H. sapiens)

Chicken (G. gallus)

Anolis (A. carolinensis)

Xenopus (X. laevis)

Coelacanth (L. chalumnae)

Spotted gar (L. occulatus)

Zebrafish (D. rerio)

Medaka (O. latipes)
Chr. $3{ }_{192,79}^{\text {Mb21d2 }}$

Chr. $9::_{13,30}^{\text {Mb21d2 }}-\left.\sum_{13,36}^{\text {Fgf12 }}\right|_{13,90} ^{\text {IIrap }}$

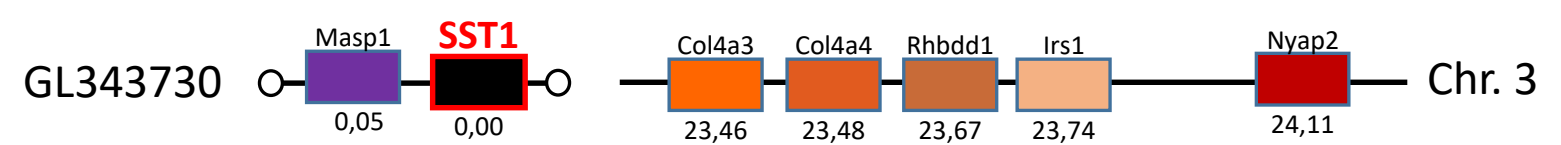

GL172717 $-\sum_{0,27}^{\text {Mb21d2 }}$

$\mathrm{JH} 126687-\sum_{2,29}^{\text {Mb21d2 }}$

LG14 $-\sum_{18,86}^{\text {Mb21d2 }}$

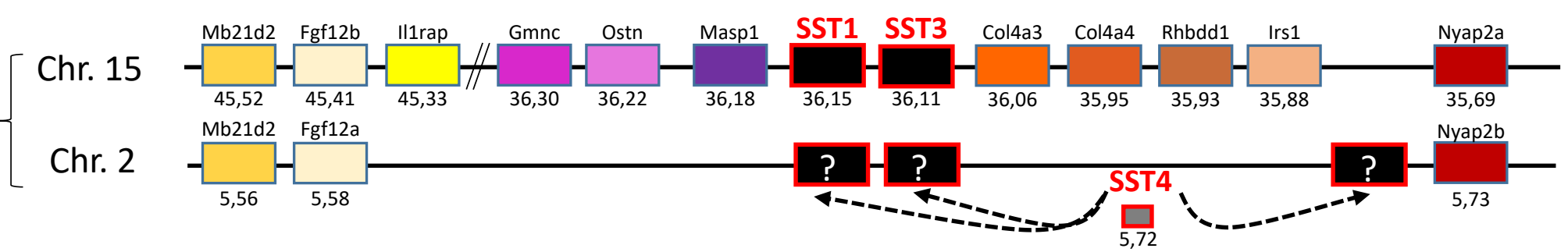

Sc_660 O-O

Chr. 17

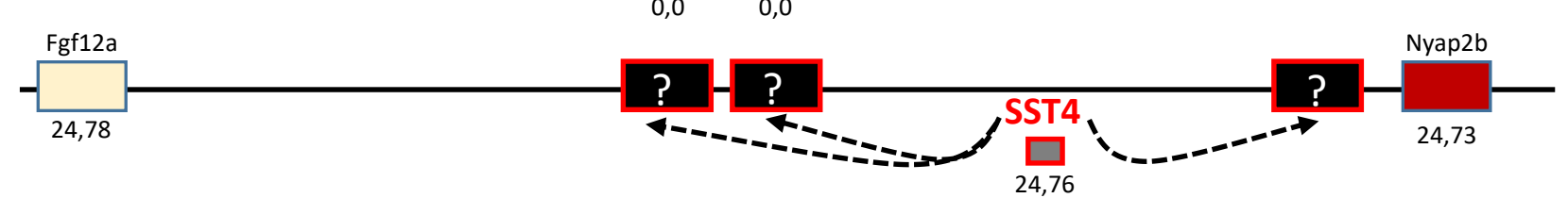


Whale shark (R. typus)

Elephant shark (C. milii)

Human (H. sapiens)

Chicken (G. gallus)

Anolis (A. carolinensis)

Xenopus (X. tropicalis)

Coelacanth (L. chalumnae)

Spotted gar (L. occulatus)

Zebrafish (D. rerio)

Medaka (O. latipes)
Sc. NW_018077477 O-

O-

Sc. 93 Ube4b
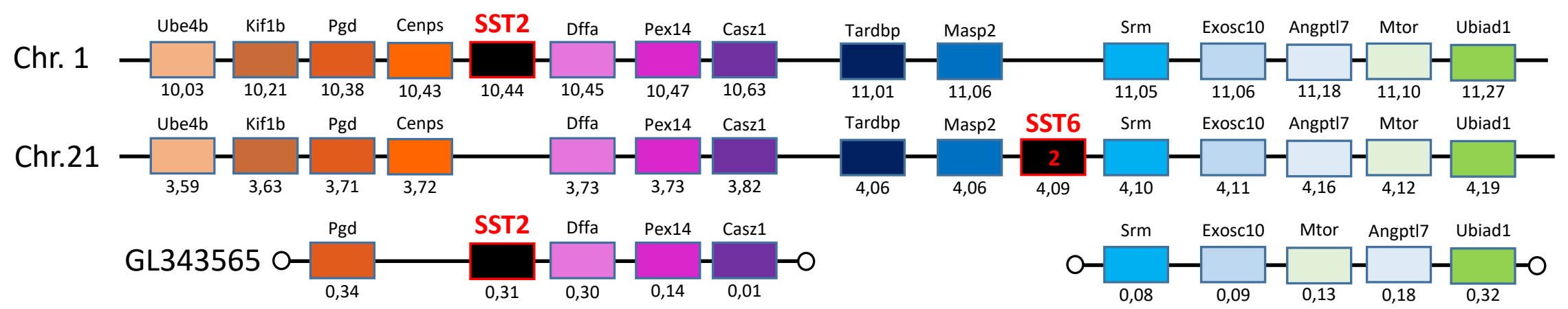

Srm Exosc10 Mtor Angpt17 Ubiad1

GL343615

GL173138 $\mathrm{JH} 127248$ -

LG25 $-\sum_{12,34}^{\text {Ube4b }} \sum_{12,24}^{\text {Kif1b }}$

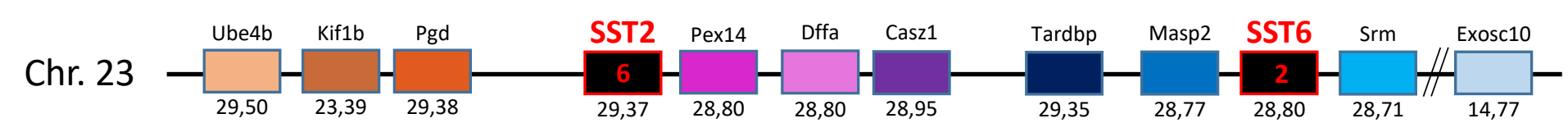

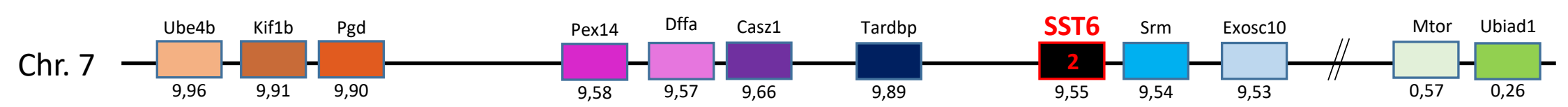

Figure 3 


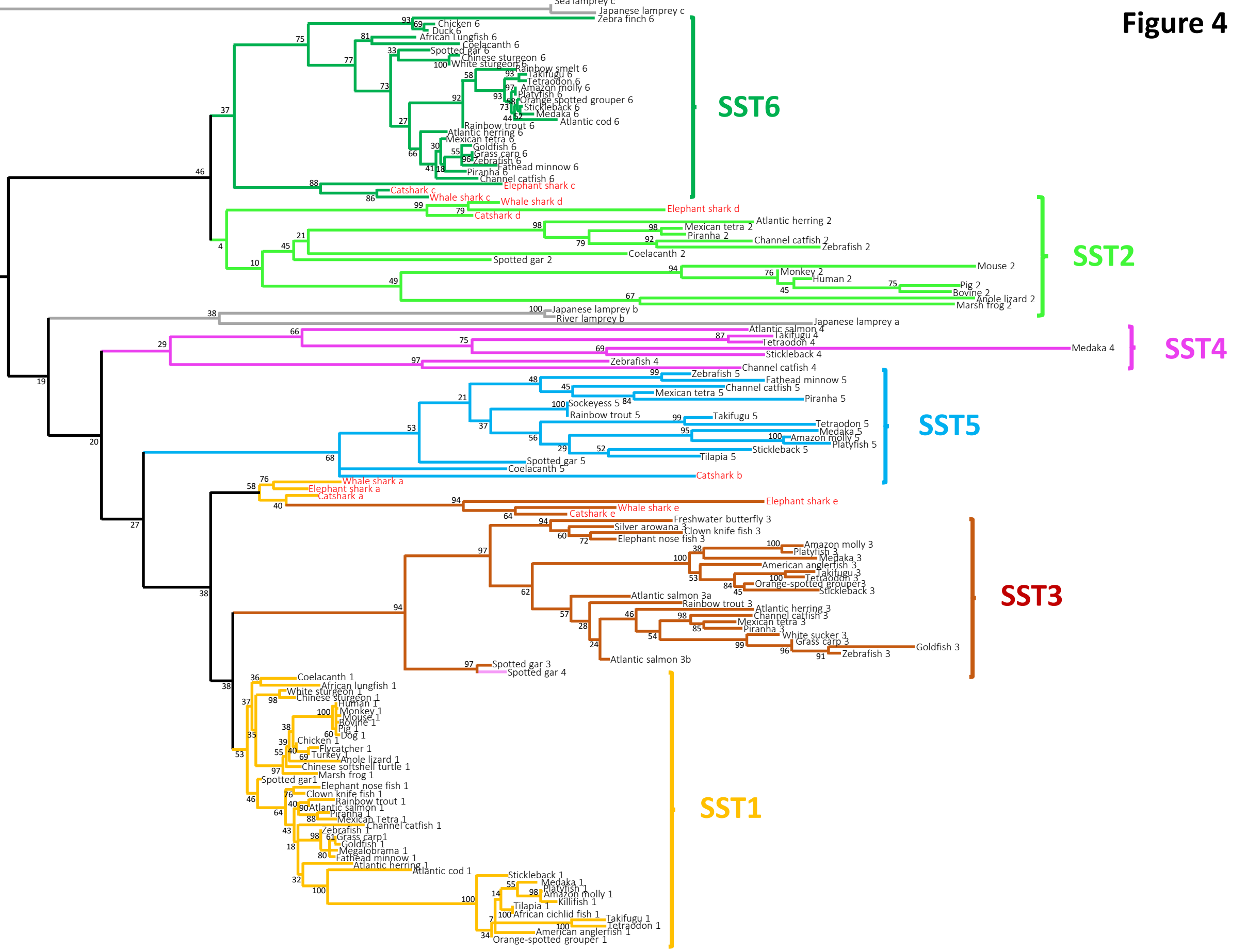




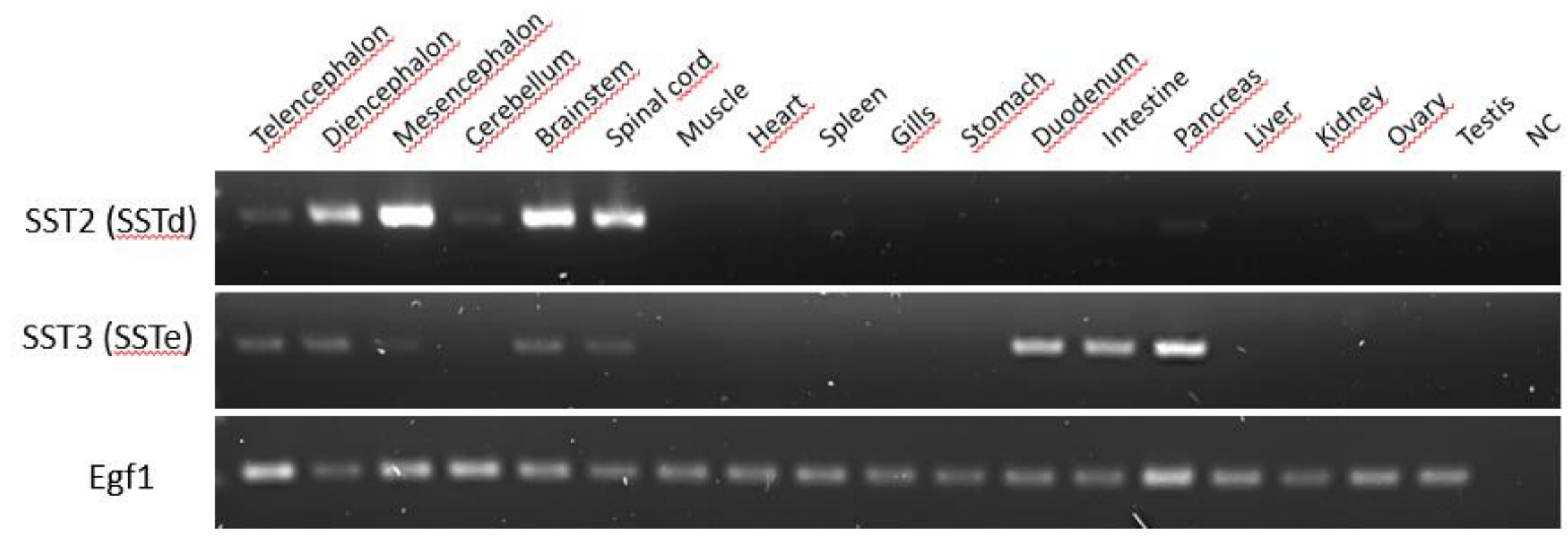




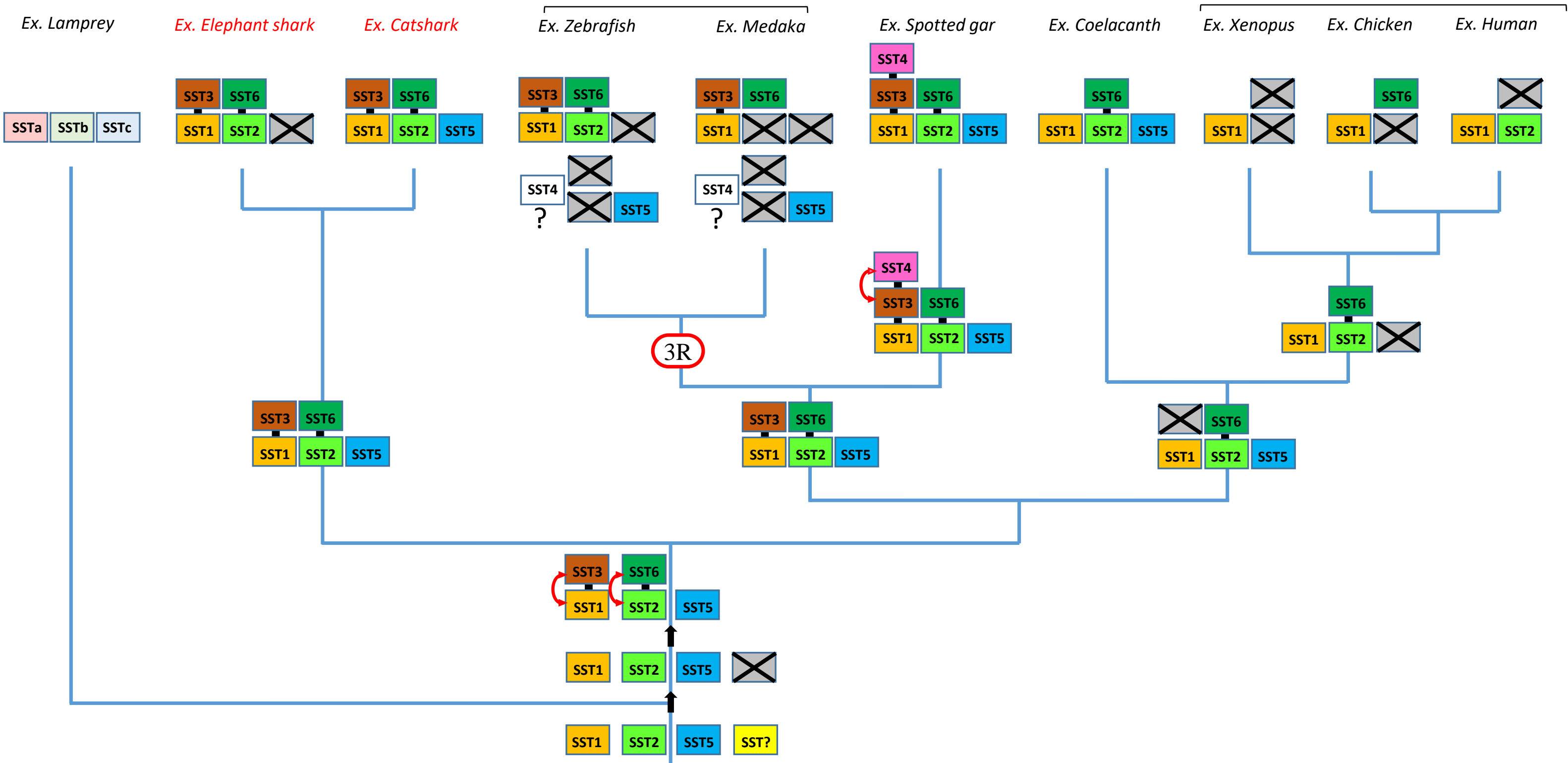




\section{Figure S1}

\section{Somatostatin 1}

\section{Primates}

$>$ Human (Homo sapiens) SST1 (BC032625)

MLSCRLQCALAALSIVLALGCVTGAPSDPRLRQFLQKSLAAAAGKQELAKYFLAELLSEPN QTENDALEPEDLSQAAEQDEMRLELQRSANSNPAMAPRERKAGCKNFFWKTFTSC >Monkey (Macaca fascicularis) SST1 (XP_005545499)

MLSCRLQCALAALSIVLALGGVTGAPSDPRLRQFLQKSLAAAAGKQELAKYFLAELLSEPN QTENDALEPEDLSQAAEQDEMRLELQRSANSNPAMAPRERKAGCKNFFWKTFTSC Other mammals

>Bovine (Bos taurus) SST1 (NP_776385.1)

MLSCRLQCALAALSIVLALGGVTGAPSDPRLRQFLQKSLAAAAGKQELAKYFLAELLSEPN QTEIDALEPEDLSQAAEQDEMRLELQRSANSNPAMAPRERKAGCKNFFWKTFTSC

$>$ Dog (Canis lupus familiaris) SST1 (NP_001003307.1)

MLSCRLQCALAALSIVLALGGVTCAPSDPRLRQFLQKSLAAAAGKQELAKYFLAELLSEPN QTENDALEPEDLSQAAEQDEMRLELQRSANSNPAMAPRERKAGCKNFFWKTFTSC

>Mouse (Mus musculus) SST1 (NP_033241.1)

MLSCRLQCALAALCIVLALGGVTGAPSDPRLRQFLQKSLAAATGKQELAKYFLAELLSEPN QTENDALEPEDLPQAAEQDEMRLELQRSANSNPAMAPRERKAGCKNFFWKTFTSC

>Pig SS1 (Sus scrofa) SST1 (AAT02532.1)

MLSCRLQCALAALSIVLALGGVTGAPSDPRLRQFLQKSLAAAAGKQELAKYFLAELLSEPN QTENDALEPEDLSQAAEQDEMRLELQRSANSNPAMAPRERKAGCKNFFWKTFTSC

\section{Birds}

>Flycatcher (Ficedula albicollis) SST1 (ENSFALT00000007767.1)

MLSCRLQCALALLSIALALGTVSAAPSDPRLRQFLQKSLGGGGGGGELAKYFLAELLSEPSQ TENEALESEDLSRGAEQDEVRLELERSANSNPALAPRERKAGCKNFFWKTFTSC

$>$ Chicken (Gallus gallus) SST1 (CN210817.1)

MLSCRLQCALALLSIALAVGTVSAAPSDPRLRQFLQKSLAAAAGKQELAKYFLAELLSEPSQ TENEALESEDLSRGAEQDEVRLELERSANSNPALAPRERKAGCKNFFWKTFTSC

$>$ Turkey (Meleagris gallopavo) SST1 (ENSMGAT00000008507.2)

KELAKYFLAELLSEPSQTENEALESEDLSRGAEQDEVRLELERSANSNPALAPRERKAGCKN FFWKTFTSC

\section{Reptiles}

>Anole lizard (Anolis carolinensis) SST1 (XP_003228868)

MLPCRLPCALALLSLALALGSGAAAPADPRLRQFLQKSLAAAAAGKQELAKYFLAELLSEP GQAENEALEPEELSRGGGAEQDEVRLELERSASLNPALEPRVRKAGCKNFFWKTFTSC

$>$ Chinese softshell turtle (Pelodiscus sinensis) SST1 (ENSPSIT00000016437.1)

MLSCRLQCALALLSLALAVSSVSGAPSDPRLRQFLQKSLAAAAGKQELAKYFLAELLAEPS QPENEALEPEDLSRGPEQDEVRLELERSANPNPALAPRERKAGCKNFFWKTFTSC

Amphibians

$>$ Marsh frog (Pelophylax ridibundus) SST1 (P87384.1)

MQSCRVQCALTLLSLALAINSISAAPTDPRLRQFLQKSLASAGKQELAKYFLAELLSEPSQTD NEALESDDLPRGAEQDEVRLELERSANSSPALAPRERKAGCKNFFWKTFTSC

Lobe-finned fish

>Coelacanth (Latimeria chalumnae) SST1 (XP_005992512.1)

MLSSRLQCAFAFLSLALVVSNISAAPSDLRLRQLLQKSLAAAAGKQEVSKYSLAELLAELAQ AENEALESDDFSKTSDQDEVRLELERSANGNPALAPRERKAGCKNFFWKTFTSC

>African lungfish (Protopterus annectens) SST1 (AAD39138.1) 
MLSCRFQCALVLLSLAVVFSKVSAAPSDLRLRQLLQRSLAAAAGKQELTKYSLAELLSELA QSENDALDSSDLSRGADQDEVRLELDRSANSSPLAARERKAGCKNFFWKTFTSC

\section{Euteleosts}

$>$ Orange-spotted grouper (Epinephelus coioides) SST1 (AAU93565.1)

MKMVSSSRLRCLLLLLLSLTASISCSSAAQRDSKLRLLLHRTPLLGSKQDMSRASLAELLLSD LLQVENEALEEENFPLAEGEPEDVHVDLERAAAAGSGPLLAPRERKAGCKNFFWKTFTSC

$>$ Killifish (Fundulus heteroclitus) SST1 (EV461374.1)

MKMFSSLSLRCLLLLLLCLSASISCSAAAQRDSKLRLLLHRTPLLSSQQDVSRSSLAELLLSD LLQMENEALEEDGFTLPEGEPEDIRVDLERAASSGPLLAPRERKAGCKNFFWKTFTSC $>$ Atlantic cod (Gadus morhua) SST1 (AAZ85702.1)

MMSVRSTLLLLSCAAMLSGSSAAPSDTKLRQLLQRSLLAPMPGQEETVRYTLAQLLSELAQ AENEALGTDDQDDERMDLERAAGPMMAPRERKAGCKNFFWKTFTSC

>Stickleback (Gasterosteus aculeatus) SST1 (KT235748)

MVSSRLRCLLLLLLSLTASISCSSTAQRDGKLHLLLHRTPLLSSKQDMSRASLVELLLSDLLQ VQNEALEEENLAPAGGEPEEVHLDLERAADAGPLLGPRERKAGCKNFFWKTFTSC

>African cichlidfish (Haplochromis burtoni) SST1 (AAS97964.1)

MNSSSRLRCLLLLLVSLTASISCTSAAQRDSKLRLLLHRTPLLGSKQDMSRSSLAELLLSDLL QVENEALEEENFPLADGEPEDIRVDLERAAGSGPLLAPRERKAGCKNFFWKTFTSC

>American anglerfish (Lophius americanus) SST1 (P01169.1)

MKMVSSSRLRCLLVLLLSLTASISCSFAGQRDSKLRLLLHRYPLQGSKQDMTRSALAELLLS DLLQGENEALEEENFPLAEGGPEDAHADLERAASGGPLLAPRERKAGCKNFFWKTFTSC

$>$ Rainbow trout (Oncorhynchus mykiss) SST1 (CA364601.1)

MLSTRVQCALALLSLALAISSVSAAPSDAKLRQLLQRSLMAPAGKQELARNTLVELLSELAH VENEAIELDDMSHGVEQEDVDLELERAPGPVLAPRERKAGCKNFFWKTFTSC

> Tilapia (Oreochromis niloticus) SST1 (ENSONIT00000001338.1)

MNSSSRLRCLLLLLVSLTASISCTSAAQRDSKLRLLLHRTPLLGSKQDMSRSSLAELLLSDLL QVENEALEEENFPLADGEPEDIRVDLERAAGSGPLLAPRERKAGCKNFFWKTFTSC

>Medaka (Oryzias latipes) SST1 (XP_004084505.1)

MMSSSSRLLLLLLSLTASISCSSAAQRDSKLRLLLQRTPLLGSKQDMSRSSLAELLLSDLLQM ENEVLEEDGFPLSDGEPEDVRVDLERAAASGPLLAPRDRKAGCKNFFWKTFTSC

>Amazon molly (Poecilia formosa) SST1 (ENSPFOT00000005658.1)

MKMLSSLSLRCLLLLLLSLSASISCSSAAQRDSKLRLLLHRTPLLSSKQDASRSSLAELLLSDL LQMENEALEEDGFGLPEGEPEDIRVDLERAASSGPLLAPRERKAGCKNFFWKTFTSC

$>$ Atlantic salmon (Salmo salar) SST1 (CB509644.1)

MLSTRVQCALALLSLALAISSVSAAPSDAKLRQLLQRSLMAPAGKQELARYTLAELLSELAQ AENEAIESDDLSRGVEQEDVRLELERAAGPVLAPRERKAGCKNFFWKTFTSC

> Takifugu (Takifugu rubripes) SST1 (ENSTRUG00000010048)

MFSSNSMCLLMLLLSLSASFSCSSAAHRDFKLPLLLQSNALMGNKQEMTRSSLANLLLSDLL QVGNEALEENLALAEGEPEEVHIDMERAAAAGIGPFLTPRERKAGCKNFFWKTFTSC

> Tetraodon (Tetraodon nigroviridis) SST1 (KT235747)

MKMFSNSCCLLMLLLSLSASFSCSSAAHRDSKLALMLPPNPLLGHRQEMSRSSLADLLLSDL LQVENQALDENFPLVEGEPEEVHVDMERAADAGIGPFLTPRERKAGCKNFFWKTFTSC

>Platyfish (Xiphophorus maculatus) SST1 (ENSXMAT00000014478.1)

MKMLSSLSLRCLLLLLLSLSASISCSSAAQRDSKLRLLLHRTPLLSSKQDASRSSLAELLLSDL LQMENEALEEDGFPEGEPEDIRVDLERAASSGPLLPRERKASCKNFFWKTFTSC

\section{Other teleosts}

>Mexican tetra (Astyanax mexicanus) SST1 (ENSAMXT00000006698.1)

MLSTRVQCALALLSIALAVGSVSGAPSDAKLRQFLQRSLIGSAGKQELARYTLAELLSELAQ AENEALEDDVPRGAEQEDVRMELERAAGPVLAPRERKAGCKNFFWKTFTSC 
>Goldfish (Carassius auratus) SST1 (Q9YGH5.1)

MLSTRIQCALALLSLALAVCSVSAAPTDAKLRQLLQRSLLNPAGKQELARYTLADLLSELVQ AENEALEPEDLSRAVEKDEVRLELERAAGPMLAPRERKAGCKNFFWKTFTSC

$>$ Clown knife fish (Chitala chitala) SST1 (AAK97070.2)

MLSTRIQCALALLSLALAVSSVYAAPSDLKLRQLLQRSIIAPASKQELARYTLAELLSELAQV ENEALESDDLSRGADQEEVRLELERAAGPPLAPRERKAGCKNFFWKTFTSC

>Atlantic herring (Clupea harengus) SST1 (XP_012673158.1)

MLSLRLQCALALLSIVLAISCISAAPTDVKLRQLLQRSLFAPGGKQELARLTLAELLSELAQA ENEALESEDMSRGAEGEDVRYELERSAGSMLAPRERKAGCKNFFWKTFTSC

> Grass carp (Ctenopharyngodon idella) SST1 (ACB69423.1)

MLSTRIQCALALLSLALAVCSVSAAPTDAKLRQLLQRSLLNPAGKQELARYTLADLLSDLV

QAENEALEPEDLSRAVEKDDVRLELERAAGPMLAPRERKAGCKNFFWKTFTSC

>Zebrafish (Danio rerio) SST1 (AAH76254.1)

MLSTRIQCALALLSLALAVSSVSAAPSDAKLRQLLQRSLLSPAGKQELARYTLADLLSDLVQ AENEALEPEDLSRGAEKDDVRLELERAAGPMLAPRERKAGCKNFFWKTFTSC

>Elephant nose fish (Gnathonemus petersii) SST1 (AF292652)

MLSSRIQCALALLSLALAVSSVSAAPSDLKLRQLLQRSLLAPASKQDLARNPLEELLSEMVR VENEALEPDDLSRGADQEEVRLELERAAGPALAPRERKAGCKNFFWKTFTSC

$>$ Channel catfish (Ictalurus punctatus) SST1 (CAA23877.1)

MPSTRIQCALALLAVALSVCSVSGAPSDAKLRQFLQRSILAPSVKQELTRYTLAELLAELAQ

AENEVLDSDEVSRAAESEGARLEMERAAGPMLAPRERKAGCKNFFWKTFTSC

>Megalobrama (Megalobrama pellegrini) SST1 (AAO92644.1)

MLSTRIQCALALLSLALAVSSVSAAPSDAKLRQLLQRSLLNPAGKQELARYTLTDLLSDLVQ AENEVLEPEDLSRAVEKDEVRLELERAAGPMLAPRERKAGCKNFFWKTFTSC

>Fathead minnow (Pimephales promelas) SST1 (DT100930)

MLSTRIQCALALLSLALAISSVSAAPSDAKLRQLLQRSLLNPAGKQELARYTLADLLSELVQ AENEALEPEDLSRAVEKDDVRLELERAAGPMLAPRERKAGCKNFFWKTFTSC

$>$ Red-bellied piranha (Pygocentrus nattereri) SST1 (XP_017539070.1)

MLSTRVQCALALLSLALALSSVSGAPSDAKLRQFLQRSLIGPSGKQELARYTLAELLSELAQ AENEALEAEDMPRGAEQEDVRMELERAAGPVLAPRERKAGCKNFFWKTFTSC

Other ray-finned fish

>White sturgeon (Acipenser transmontanus) SST1 (AAL13248.1)

MLSSRLQCALAFLSLALAVSSVSAAPSDPRLRQLLQRTLAASAGKQELLKYSIAELLSELAQS ENDALASDELSRAAEQNDVRLELERSANGNPAMAPRERKAGCKNFFWKTFTSC

>Chinese sturgeon (Acipenser sinensis) SST1 (FJ792687.1)

MLSSRLQCALALLSLALAVSSVSAATSEPRIRQLLQRALVASAGKQDLLKYSLAELLSELAQ SENDALASDELSRAAEQNDVRLELERSANGNPAMAPRERKAGCKNFFWKTFTSC

>Spotted gar (Lepisosteus oculatus) SST1 (XP_006637937.1)

MLSSRVQCALALLCLALAVSSVSAAPSDLRLRQLLQRSLAASAGKQELARYTLAELLSELA QSENEALESDDLSRAAEQDEVRLELERSANPALAPRERKAGCKNFFWKTFTSC

\section{Cartilagonous fish}

>Elephant shark (Callorhinchus milii) SSTa (XP_007910586.1)

MSCGRVQCALALLSIALTVLSVTSAPAHDRYREILQRSLAAAGARSKPELTKYSLAQLLAEL ANAENEALEAEDMARATAQDEVRVELERSANPNLAQRERKAGCKSFFWKTFTSC

>Whale shark (Rhincodon typus) SSTa (XP_020365362.1)

MWCSRVQCALALLSIALAVLSAGAAPTADRYRELLQTSMAGAEPRSKAELIKYNLVQLLAE LANAENEVLDGEDLPRMAKQDEVRVDLERSANPNSPQRERKAGCKNFFWKTFTSC

$>$ Catshark (Scylorhinus canicula) SSTa (JX964750)

MWCSRLQLSLALLSIALAVLSVSSAPTDNRYREILQRAMAATGSGGKAELTKYSLAQLLSEL ENAENEALETDAMGGRNEVRLELERSINPNLANRERKAGCKNFFWKTFTSC 


\section{Somatostatin 2}

\section{Primates}

>Human (H. sapiens) SST2 (AAB66895.1)

MPLSPGLLLLLLSGATATAALPLEGGPTGRDSEHMQEAAGIRKSSLLTFLAWWFEWTSQAS AGPLIGEEAREVARRQEGAPPQQSARRDRMPCRNFFWKTFSSCK

> Monkey (M. fascicularis) SST2 (XP_015298230)

MPLPPGLLLLLLSGATATAALPLEGSPTGPDSEHMQEAAGIRKSSLLTFLAWWFEWTSQASA GPLIGEEAREVARRQEGAPPQQSARRDRTPCKNFFWKTFSSCK

\section{Other mammals}

> Bovine (B. taurus) SST2 (NP_001191825)

MLPLCLLLPLLLLPSGATTALSPEGGLAGHESGHMQEVAEIKTNSLLTFLAWWYEWASQAR AVPFVGGEAREVSKRQEGAPLHQSTRQDKTPCKNFFWKTFSSCK

$>$ Mouse (M.musculus) SST2 (AAD51127.1)

MMGGRGTGGKWPSAFGLLLLWGVAASALPLESGPTGQDSVQEATEGRSGLLTFLAWWHE WASQASSSTPVGGGTPGLSKSQERPPPQQPPHLDKKPCKNFFWKTFSSCK

$>$ Pig (S. scrofa) SST2 (NP_001191865)

MLPPLCLLLLLLLPPLGATTALPLEGGLTSHDRDSGHMQEVAEIKKNSLLTFLAWWHEWMS QAREVPFVGGETREMPKKQEGLPRQQASRGDRTPCGNFFWKTFSSCK

\section{Reptiles}

>Anole lizard (A. carolinensis) SST2 (XP_008119733.1)

MAFLVHPPPSAPFLLALLSFSRLMGAAPAAVLPIPGGLAWKHNEGSADLGRDELLAFLSGLV DRASRRHGMPLSKQEKVVRSPREERVPFFRHPVREKAPCKNFFWKTFSSC

\section{Amphibians}

> Marsh Frog (P.ridibundus) SST2 (AAC60094.1)

MLGSAGTLLLLLLAWGARALSQPDDNRITTGRNQDLNAIQQDLLLKLLSGWTDSRESNLVE VERNVPDPPEPKIPPSVKFPRLSLRERKAPCKNFFWKTFTMC

\section{Lobe-finned fish}

$>$ Coelacanth (L. chalumnae) SST2 (NW_005819697)

MQPLILLVPLVLMAWNLGVAAVPKEERLMLYDKVNRETTGFGEERKQFLSNMLDALIGLN TLENDTPLLGTKEQEEHNLSERSAFKYSSLKKAPCRNFFWKTFSSC

\section{Teleosts}

$>$ Mexican tetra (A. mexicanum) SST2 (ENSAMXT00000013558.1)

MALSGGGDTCGAADGGMFIMRMLLSLVPLLLIAWSGTNTDALPIDNKLPQSKEVLTKEQKD LLIKIMSDLAELNMTLKDLGALDLEQLLNGKLGERSVEKSPCTNFFWKTFSAC

$>$ Atlantic herring $(C$. harengus) SST2 (XP_012681486.1)

MGVLARVDPCSVAQSARITMRVLTSLVPLILIVWSGSQTGALPVREKPLRNESLADEERILLL KILAGLTEKNLTEENLTGHDLEGLLNGTLKGGSVPTPQERSPCKNFFWKTFSSC

$>$ Zebrafish (D. rerio) SST2 (ENSDART00000146411.2)

MFLMRVLLSLFPLVFVIWNGEALPIQDKQSQTNEILSKEEDGLKMITYVAELNLTSKELADL DAELLNGKLWEKSDKPSKDKSPCKLFFWKTFSSC

$>$ Channel catfish (I. punctatus) SST2 (XP_017341959.1)

MALGGRINLYQAVDSPMFTMRVLLSLIPLLFVAWSANNADALPVQDKLPNSNEVLTKEQK DFMTQFLANLAELNMTIKDLETMDHEQLSKLSERAAFGLPPAREKSQCNFFWKTFSSC

$>$ Red-bellied piranha (P. nattereri) SST2 (XM_017706353.1)

MALAVGGTSCGGADGGMFIMRMLLSLVPLLLIAWSGTSTDALPVENKLPQSNEVLTKEQK DLLIKIMSDLAELNMTVKDLGALDLEQLLSGKLGERSVFGLPAQEKSPCKNFFWKTFSACQ $\mathrm{H}$ 


\section{Other ray-finned fish}

>Spotted gar (L. oculatus) SST2 (NC_023203.1)

MHLLSSLVPLALLVWSLKCAAAVPSEERRALQSNGGLAKERKDVLLKVLSGLLVSNLLRKE QSAVDSEQLKSSLMGERSVFGPSVSRERTPCKNFFWKTFSSC

\section{Cartilaginous fish}

>Elephant shark (C. milii) SSTd (XP_007900347.1)

MQWQAFASVVCLLFIVTVQGVEPQEERMGLQLNREMVKDKKRLIAKLLAGLLDVDDNLLE SVIAPLGLSKEEGTDFEERSAAGEIPPRKPKAGCKLFFWKTFSHC

>Whale shark (R. typus) SSTd (XP_020390988.1)

MTVCLMSLLLLLSVEAADPLEERMKLQVNREVTKSRKYLMLKLLSGLLDPDDNLLENDFTS LSPGEEESSLEERSVNAGIPRRPQKAPCKLFFWKTFSHC

$>$ Catshark (S. canicula) SSTd (MK491629)

MKWLMTVSLMSLLYLLRVEGTDLLEERMKLQVNREMTKSRKNLIVKLLAGLLETDDNLLE NGFAPLKPEEAEGTLFEERSVNAGIPRREQKTPCKLFFWKTFSHC

\section{Somatostatin 3}

\section{Euteleosts}

$>$ Orange-spotted grouper (E. coioides) SST3 (AAU93566.1)

MQCIRCPTILVLVALVLCSPGVFSQPDRDQDQYQNQDLDLELRHHRLLQRARSAGLLSQEW SKRAVEDLLAQMSLPEADTQREAEVVSMATGGRMNLERSVDPPNNLPPRERKAGCKNFYW KGFTSC

>Stickleback (G. aculeatus) SST3 (KT235756)

MQYTRCPAILVLVGLVLCGPGVSSQRNRDQDQDQYQNQDLDPRPHLLLQRARGAGLLSQD WSKRAVEDLLAQMSLPEADSQLGAEVVSAATGGRLHLERSVDPPNNLPARERKAGCKNFY WKGFTSC

>American anglerfish (L. Americanus) SST3 (P01170.2)

MQCIRCPAILALLALVLCGPSVSSQLDREQSDNQDLDLELRQHWLLERARSAGLLSQEWSK RAVEELLAQMSLPEADVQREAEDASMATGGRMNLERSVDSTNNLPPRERKAGCKNFYWK GFTSC

>Rainbow trout (O. mykiss) SST3 (Q91194.1)

MKVCRIHCALALLGLALAICSQGAASQPDLDLRSRRLLQRARAAAWPHRSGVSERWRTFYP NCPCLRPRKVKCPAGAKEDLRVELERSVGNPNNLPPRERKAGCKNFYWKGFTSC

>Medaka (O. latipes) SST3 (AU168379.1)

MQHARGPAFVVLAALALSSLGVSSQSDRDQDQFQNHDLDLELRHHRLLQRARSAGLLSQD WSKRAIENMLARMPDPEAEAEREAEVGRNNMERSVDANVPPRNRKDGCKNFYWKGFTSC >Amazon molly ( $P$. formosa) SST3 (ENSPFOT00000005672.2)

MHHARCPTILVLAALAALSLGVSSQADRDQDPYQNQNLDLDLELRHHRLLQRARSAGLLS QDWSKRAVEDLLAQMSRSAVEAQQDDPEAASKGTDSRLNLERSVETTARERKAGCKNFY WKGLTSC

>Atlantic salmon (S. salar) SST3a (DW531645.1)

MKFSQIHCALALLGLALAICSQGAASQPDLDLRSRRLLQRARAASIATQEWSKRVMEDVLS KLSLPDVEAQKSVGSTADAKEDLERSVDNSNNLPPRERKAGCKNFYWKGFTSC

$>$ Atlantic salmon (S. salar) SST3b (CB505007.1) 
MKVCRIHCALALLGLALAICSQGAASQPDLDLRSRRLLQRARAAGIATQEWSKRAVEDILS QLSWPEAQESEVSPAGAKEDLRVELERSVGNPNNLPPRERKAGCKNFYWKGFTSC

$>$ Takifugu (T. rubripes) SST3 (ENSTRUT00000025350.1)

MQRIHSSTILMLTTLVLCIQGVSSQPDRDLNQNQDLEMEVRHHRLLQHVHGAGLLSQEWSK RAVEDLIAQMSMPEGTGQRDTEVVSMATGGRMNLERSVDTTNNLSPPERKAGCKNFYWK GPTSC

>Tetraodon (T. nigroviridis) SST3 (ENSTNIT00000001563.1)

MPGVRSSTILVLTALVLSIQAVSSQPDRDLDQNQDLDQEVRRHRLLQRAHGAGLLSQDWSK RAVEDLIAQMSLPEGTGQRDTEVVSMATGGRMNLERSVDTTNNLPPRKAGCKNFYWKGFT $\mathrm{SC}$

>Platyfish (X. maculatus) SST3

MHHARYPAILVLAALAALSLGVSSQADRDQDPYQNQNLDLDLELRHHRLLQRARSAGLLS QDWSKRAVEDLLAQMSRSEVEAQQDGPEAASRGTGSRLNLERSVETTARERKAGCKNFY

WKGLTSC

Other teleosts

>Mexican tetra (A. mexicanum) SST3 (ENSAMXT00000006711.1)

MKVCVLQCGLVLLGLALVLCSSGASSQPDLDLDLDVDLRHRRLLQRARALGIAAQEWKKR EMEDLLSQLTLPESEFQESEISTLGSKQDVRVEMDRSAENPNSLPPRERKAGCKNFYWKGFT SC

$>$ Goldfish (C. auratus) SST3 (AAF15306.1)

MRLCELHCYLALLGLSLVLCGRCANSQLEPDLDFRHHRLLQRASATGQATRWDFTKRDVE KLLSLLSIPEMEMREKGLSMAGESEDLRLEQERSAESSNQLPTRVRKEGCKNFYWKGFTSC >White sucker (Catostomus commersonii) SST3 (AAK97071.2)

MRLCELHCYLALLGLSLVLCDRGADSQLEPDMDFRHRRLLQRARAIGLATQDWTKKDIEEL LSQLSLPEIEARENGVSTTGGNDDLHLELERSAENTNQLYPRERKAGCKNFYWKGFTSC

$>$ Clown knife fish (C. chitala) SST3 (AAV48555.1)

MKNCQAQCILALMGLFLGLCWPSDASQPDLRYRSLLQRAHSAPASPQDWTRRTVEDLLAR LPPPEGEFTPVEDVPAADEGEEVRVELERSVDPNNLPPRERKAGCKNFYWKGFTSC

>Atlantic herring (C. harengus) SST3 (XP_012693360.1)

MMRFSGIHCSLALLGLSLVLCSHSASSQPDLDLDLDLRHHRLLQRARAAGMSTQEWSKRAV EDLLSQLSLPEEETQDREVSTVGAAKDDLHMERSAETNNIPPKDRKSGCKNFFWKGRTSC

$>$ Grass carp (C. idella) SST3 (ACB69424.1)

MRLCELQCYLALLGLSLVLCGRSANSQLEPDLDFRHRRLLQRARAIGQATQDWTKKDVEEL LSLLSMPEMEMRENDISTVDENEDLRVELERSAESSNHIPARERKAGCKNFYWKGFTSC

>Zebrafish (D. rerio) SST3 (XP_694143.1)

MRLCELQCYLALLGLSLVLCGRSANSQLEPEMDFRHHRLLQRARAIGQAQEWTKKDVEEL LSLLSMPEMQMRESDLSTTDENEDLRVELERSAESSNHIPARERKAGCKNFYWKGFTSC

$>$ Channel catfish (I. punctatus) SST3 (CK424149.1)

MRTCVLQCCLTLLGLSLVLCSNGVSSQPDLDLRHRRLLQRVQALGVAAQEWTKNDLKAIL AQLTMPESEFQESDVSTLGTKQDLTVELERSAEDPNSLPPRERKAGCKNFYWKGFTSC

$>$ Elephant nose fish (G. petersii) SST3 (AAV48556.1)

MKTCQVHCVLVLLGLVLGLCYPSAASQPDLRYRSFMQRAHTAAMSPQDWSRRVVEDLLSR LAPPEDDTPQSEMSTAEEGEETRVELERSVEPNNLPPRERKAGCKNFYWKGFTSC

>Silver arowana (Osteoglossum bicirrhosum) SST3 (AAK97067.1)

MKICQIHCTLVLLGLVLGLYCPSAASQPDLRYRSFLQRAHAAAMSPQDWSKQAVEELLSRL APAQGEVPQGAVSAADEEEDVRVDLERSLELNNLPPRERKAGCKNFYWKGFTSC

>Freshwater butterfly fish (Pantodon buchholzi) SST3 (AAK97068.2)

MKLCQVHCILALLGLVLGMCGSSSATQLDSRYRSLVQRARAASMGPQQDWGKLSVEDLSL LAATEADMPFGDMSAAEESEGAHLDLERSVEPGNVPPRERKAGCKNFYWKGFTSC 
$>$ Red-bellied piranha (P. nattereri) SST3 (XP_017539071.1)

MRVCVLQCCLALLGLSLVLCGNGASSQPDLDMDVDVDLDPRYHRLLQRARALGMAAQD WRKREMEDLLSQLTLPESEFKESEITSLGAKHDVRVELERSAENPNNLPPRERKAGCKNFY WKGFTSC

Other ray-finned fish

>Spotted gar (L. oculatus) SST3 (ENSLOCT00000011545.1)

MLSYRVHCTLALFGLVLAFCCVSAVSHSDLRFRRFLQKAKAAGLNKQDSSKYAIEDLISQL VQSDSEALENEMSVLDENSDARLELERSTEIGKEIPPRERKAGCRNFYWKTFTSC

\section{Cartilaginous fish}

>Elephant shark (C. milii) SSTe (XP_007910587.1)

MQARCTLTLALLSLAALGLSALPTHLRDQLVLQGSPAMELRDGVVRELIQAVADAESKPQS EEAAEDFAPDEKAELRLALAPRWRKDCKNFFWKTYTLC

>Whale shark (partial) (R. typus) SSTe (XP_020365363.1)

DFLKDTIIQLLHVVADADKDAVEVNASQVETPPXNKIPMKFQQRQLGTRMRKNCKNFFWK TYTLC

>Catshark (S. canicula) SSTe (MK491630)

MWCSRLQLSLALLSIALAVLSVSSAPTDNRHREILQRALDQELLKDTMMKLLPLVTDVDTE AGTLDVSNAETSEENTTPMKFQHRQLGMRMRKNCKNFFWKTYTLC

\section{Somatostatin 4}

\section{Euteleosts}

>Stickleback (G. aculeatus) SST4 (KT235754)

MAHILCVFALLCFAFCAAQDGETQRGFKDNRLQRDSLPWLDKLQDEQDSTKKLNLIEWLYS FYKFDNGNIVKGPTDAEEPEKNRRGLGKTTRRFGCRVFFWKSWSPC

$>$ Medaka (O. latipes) SST4 (KT235752)

MANVLCVLAFLCFAVCVVQNTEIMVESEDLPAGQGLLSSFDRISDFQQSVETGILMNLLYKL SRPDSAITLQQVRDSEKQEKNRRGVEIATQRPCRVFYWKSWAKC

$>$ Atlantic salmon (S. salar) SST4 (CA039604.1)

MALTGIWCAFVLVCVTLCQMGKVDSFVNEDKPTWLETLQDKQVSAADDKYNLVWLLYKL SQSHHDNTFKINFPPHGPESVGKPESRRETIPQDSRVRKEGCKVFFWKSWTAC

$>$ Takifugu (T. rubripes) SST4 (NC_018911.1)

MARALCILVLLCFAPCCAENSKTEHRFNDLQVQEDLLSWLKPQEKWEPSKRQTLEDLLYKL TNYKSENRILPQQPGDTENQEKNRRDLDNLRVQQRQFGCRMFFWKSWTYC

$>$ Tetraodon (T. nigroviridis) SST4 (KT235753)

MARALCFLVLLCFASRCAGNSKTEHRLKDLQVEEDVLPWLNSQEKWEPSKGQNLEDLLYK LTNYRSENRIVSQQPSDTNQEKNRRGLDNLKVQQRQAGCRIFFWKSWTSC

Other teleosts

>Zebrafish (D. rerio) SST4 (NP_571802.1)

MASSQLHLTATLLCLAMMAGIICGRSHMLLNSALQASRGTSADEEIPERYSLSELEWLLSNS DPAVFQPDSSSLGSLHSGLELMRRDTKEERKTGCKNYFWKSRTAC

$>$ Channel catfish (I. punctatus) SST4 (P01172.1)

MSSSPLRLALALMCLVSAVGVISCGRPHVVLNSALEEARNVPFGEEVPERLTLPELQWMLS NNELTPVQVEEAPRSRLELVRRDNTVTSKPLNCMNYFWKSRTAC

Other ray-finned fish

>Spotted gar (L. oculatus) SST4 (ENSLOCT00000011651.1)

MLSYRVHCTLALFGLVFAFCCVSAVSNSDLRFRRFLQKAKAASLSTRDSSKYALEDLISQIA QSDNEALENEMSVLDENSDARLELERSADMGKVIPPRERKAGCRNYYWKTITSC

\section{Somatostatin 5}




\section{Lobe-finned fish}

$>$ Coelacanth (L. chalumnae) SST5 (XP_005999859.1)

MMCSHVQSLLAVLSASLLLVGVGGLPKGEVLTDLFQGQGAEGKEDFSRMLMLKMVSNLL KSENDVLVPSAEDTASQDELTRRLAPSRERKAGCKNFFWKTFTSC

\section{Euteleosts}

>Stickleback (G. aculeatus) SST5 (ALD51533.1)

MQVLLVALFSSVLLVQVSGAPRGDMPTQRGDPADNKDLAYSMLLKFVSELTAARGGETLL VPEQEEEEAEGGRQEVMRRHLPLTQRERKAGCRNFFWKTFTSC

$>$ Rainbow trout (O. mykiss) SST5 (BX909872.3)

MLCSQLQVLLVALSASVFLARVSAAPHRDMLAELLRADSTKGNEDLSRTLLLKMMSDLMS

AAVGENEVLPDLEEALGVREEVRQLPLSHRERKAGCRNFFWKTFTSC

>Sockeyess (Oncorhynchus nerka) SST5 (EV380196.1)

MLCSQLQVLLVALSASVFLARVSAAPHRDMLAELLRADTTKGNEDLSRTLLLKMMSDLMS

TAVGENEVLPDLEEALGVREEVVRQLPLSHRERKAGCRNFFWKTFTSC

> Tilapia (O. niloticus) SST5 (XP_005464163.1)

MLRSQVLLVALGLSVLLVRVSTAPYSDMLTETLRADLTNDKDLTHWLLLKFMAELMAARG DETRRGREEVTRRHLSLSQRERKAGCRNFFWKTFTSC

>Medaka (O. latipes) SST5 (XP_020567335.1)

MLLAALGLSLLMACVSGAPQRNPLTHTKQLQLLKSEVDPTRLLLMKLVLELVALRRQEML QELEEEELGGRERLMKRHIRFTQRERKAGCRNFFWKTFTSC

>Amazon molly (P. formosa) SST5 (XP_007551472.1)

MLCSQMKVLFVALCSSMLALHVSSAPQMDELTETLQAELISDKDLTGLLLLRFMSELMASR GEEMLREKQEEEEEELGGRQRLMRRHIRFSHRERKAGCRNFFWKTFTSC

$>$ Takifugu (T. rubripes) SST5 (N.D.)

MLSQVQLLLVTSFSSMLLMEVSSAPRKHMPSELLPADPLKDKDLAILILLRIISEVMAAEREM LLLPQEGEEEAGVREGEVMMRRQVPFSQRDRKAGCRNFFWKTFTSC

$>$ Tetraodon (T. nigroviridis) SST5 (ALD51532.1)

MTRSQLLLAASFSFLLLMEAGGTPRKHKFLPADSVKDKDLALVTSLKFAFEGTAAQREMLL LLQEDEEDAGVREMMMMMRRQIPFSQRERKAGCRNFFWKSYTSC

$>$ Platyfish (X. maculatus) SST5 (XP_005804860.3)

MLCSQMKVLVVALCSSMLALHVSSAPQMDELTETLQAELISDKDLTGLLLLRLMSELTASR GEEMLREQEEEEEDELGGRQRLMRRHIRFTHRERKAGCRNFFWKTFTSC

Other teleosts

$>$ Mexican tetra (A. mexicanum) SST5 (XP_007243346.1)

MCSQLQVVLVALSVLVLVSRVSAAPRGDVLTQLLQNEADTKENEDISRMLMLKLLSELEVA GENEVLSGADVRNDVVRQLPFSQRERKTGCRNFFWKTFTSC

>Zebrafish (D.rerio) SST5 (XP_001333082.1)

MFSRLQVVVVTLWVSLLLCRVSSAPRGDLLFQLLRSQVDPKENELQDLSRLLLLKQLSESVT PEEKDALDSIDELDVRNEVVRQIPVSQRERKAGCRNFYWKTFTSC

$>$ Channel catfish (I. punctatus) SST5 (XP_017327476.1)

MSSQMQIVVLAVSLLVLVSRVRTAPRTDLLAHMLQNEADGKEDLSRILLLKLISELKIPDEN DTLSDDDMELNRILRHLPLTTRERKAGCRNFFWKTFTSC

$>$ Fathead minnow (P. promelas) SST5 (DT105503.1)

MFSPLQMVVATLSLSLLLCSVSSAPGGDMLIQLLRSEVEPKEIELQDFFRLLLLKQLSESAAP EEKQPRESIDDDPEVHNEVVRQIPLSHRERKTGCRNFYWKTFTSC

$>$ Red-bellied piranha (P. nattereri) SST5 (XP_017578562.1)

MNFEVKSSLLSRAAGRSASTPVIQTFEEFEYFKSICLLNHLITRVSAAPRGDILAQLQADPKG NEELSRMLILKLLSDLEIPGDNEVLSGTDVRSDMVRQLPFPQRERKTGCRNFFWKTFTSC

Other ray-finned fish

>Spotted gar (L. oculatus) SST5 (XP_006627411.2) 
MSTGQPSLPPAPAPNQGFQQHKLQVLLVALSVSVLLARVSGVPQRDRLADLLRAEVPEGKE DLSRMLLLKIFSDLLRAENEIPPGSAEDAGIRSDVPRQLPLAQRERKAGCRNFFWKTFTSC

\section{Cartilaginous fish}

$>$ Catshark (S. canicula) SSTb (JX964751)

MPRSQTQVLLTLVTVALLLLRGSSTARVQGLPDILQERNDEGTEELSRTMVLNLLSELLHAG DKGLPSTERRGLPQAEIVRRWNSPRDRKAGCKNFFWKTFTSC

\section{Somatostatin 6}

Birds

>Duck (Anas platyrhynchos) SST6

MQLVASLVSVLLLVWSVRATALPGEERLALQNSREQTKLRKDAILKMLAGLLESVDVASPD LEEEGKLEEERATLGRLAQLSQRDRKAPCKNFFWKTFTSC

>Chicken (G. gallus) SST6 (DQ279789)

MQLVASLVSVLLLVWSVRATALPGEERLALQNSREQTKLRRDALLKMLAGLLESSDVASP DLEEEGKLEEERAALGRLAQLSQRDRKAPCKNFFWKTFTSC

>Zebra finch (Taeniopygia guttata) SST6

MQLVASLASVLLLLWSVRATALPGQEGLQSTREQSTARKDVILKMLAGLLGSVDAGAEVA FPAGQEKARLEEDQAALGRLAQLSQRDRKAPCKNFFWKTFTSC

\section{Lobe-finned fish}

$>$ Coelacanth (L. chalumnae) SST6 (XP_005994063.1)

MQLLTSLVSFLLVVWSVRATALPIEEKLMSHNNVELTKERKELMLKMIAGLLEGIDSPPMG GEVGSMDLEEPVEPKLEERAAFNRLPQLPQRDRKAPCKNFFWKTFTSC

>African lungfish (P. annectens) SST6 (Q9W7E9.1)

MQFLASLVSFLLVVWSVKATALPVEDRLSVHSNRDLTREREEMILKMISGLLDGSDNSLFG GEVTSMDIEEPLENKLEERAAYNALPQLPQRDRKAPCKNFFWKTFTSC

\section{Euteleosts}

$>$ Orange-spotted grouper (E. coioides) SST6 (AAU93567.1)

MQLLVVLAALMGVLFSVRAAAVLPVEDRSSIHVNRELSKERKELILKLVSGLLDGALDTNM LPGEAAPVDLEEPLESRLEERAVYNRLSLPQRDRKAPCKNFFWKTFTSC

$>$ Atlantic cod (G. morhua) SST6

MQLMVVLAALMGVLFSVRAAALLPVEDRSNFHSSRELSKERKELILKLVSGLLEGSPDSNM LPGELSPVELEEPLESRLEERAVYNRLSLPQRDRKAPCKNFFWKTFTSC

>Stickleback (G. aculeatus) SST6 (ENSGACG00000007077)

MQLLVVLATLMGVLFSVRAAAVLPMEDRSSINVNRELSKERKELILKLVSGLLDGAADAN MLPGEAAPVDFEEPLESRLEERAVYNRLSLPQRDRKAPCKNFFWKTFTSC

$>$ Rainbow trout (O. mykiss) SST6 (CX153272.1)

MQLLVILASLMGVLYSVRAAAVLPVEERSPLLNRELSKERKELILKLVSGLLDGATDTNMLP GEGVSPVDLEEPLESRLEERAAYNRLSQLPQRDRKAPCKNFFWKTFTSC

$>$ Medaka (O. latipes) SST6 (BJ885293.1)

MQLLVVLAALMGVLFSVRAAAVLPVEDRSSIHVNRELSKERKELILKLVSGLLDGSLDTNM LPGEAAPVDFEEPLESRLEERAVYNRLSLPQRDRKAPCKNFFWKTFTSC

>Rainbow smelt (Osmerus mordax) SST6 (EL519905.1)

MQLLVVLAALMGVLYSARAAAVLPVEERSPIQANRELSTERKQLILKLVSGLLDGASDSNM LPGDVSPLDLEEPLESRLEERAVYNRLSQLPQRDRKAPCKNFFWKTFTSC

$>$ Amazon molly $(P$. formosa) SST6

MQLLVVLAALMGVLFSVRAAAVLPLEDRSPIHANRELSKERKELILKLVSGLLDGALDTNM LPGEAAPVDLEEPLESRLEERAVYNRLSLPQRDRKAPCKNFFWKTFTSC

$>$ Takifugu (T. rubripes) SST6 (ENSTRUG00000012370)

MQLLVVLAALTGVLLSIRAAAVLPVEERSPVHLNRELSKERKELILKLVSGLLDGALDTNML PMETVDLEEPLESRLEERAVYNRLSLPQRDRKAPCKNFFWKTFTSC 
>Tetraodon (T. nigroviridis) SST6 (ENSTNIG00000015178)

MQLLVVLAALTGVLLSVRAAAVLPVEERIPLHLNRELSKERKELMLKLVSGLLDGALDTNM LPMEAVDLEEPLESRLEERAVYNRLSLPQRDRKAPCKNFFWKTFTSC

$>$ Platyfish (X. maculatus) SST6

MQLLVVLAALMGVLFSVRAAAVLPLEDRSPIHANRELSKERKELILKLVSGLLDGALDTNM

LPGEAAPVDLEEPLESRLEERAVYNRLSLPQRDRKAPCKNFFWKTFTSC

Other teleosts

> Mexican tetra (Astyanax mexicanus) SST6 (ENSAMXT00000013526.1)

MQILASLVSLLLVLYSVRAAAVLPVEERSPAHTRELSKERKELILKLVSGLLDGVDNSVLGG DLVPMEAEEPLESRLEERAVYNRLSQLPQRDRKAPCKNFFWKTFTSC

$>$ Goldfish (C. auratus) SST6 (AAD09631.1)

MQLLSSLVSLLLVLYSVRAAAVLPVEERNPAQSRELSKERKELILKLISGLLDGVDNSVLDG

EIAPVPFDAEEPLESRLEERAVYNRLSQLPQRDRKAPCKNFFWKTFTSC

$>$ Atlantic herring (Clupea harengus) SST6 (XP_012681579.1)

MQLLASLVSLMLVLYSIRAAAVLPVDERSPAHARELSKERKELILKLVSGLLDGVESNMLPG DEAPLDLEEPLESRLEERAVYNRLSQLPQRDRKAPCKNFFWKTFTSC

$>$ Grass carp (C. idella) SST6 (ACB69425.1)

MQLLASLVSLLLVLYSVRAAAVLPVEERNLAQSRELSKERKELILKLISGLLDGVDNNVLAG EIAPVPLDVEEPLESRLEERAVYNRLSQLPQRDRKAPCKNFFWKTFTSC

>Zebrafish (D. rerio) SST6 (BG307388)

MQLLASLVSLLLVLYSVRAAAVLPLEERNPAQSRELSKERKELILKLISGLLDGVDNSVLAG EIAPVPLDVEEPLESRLEERAVYNRLSQLPQRDRKAPCKNFFWKTFTSC

$>$ Channel catfish (I. punctatus) SST6

MQLLASLVSLLLVLYSVRAAVVLPAEERSPAHTREPSKERKEMILKMVSSLLDGVDNNMLA GDLLPLEEEEPIDSRLEERAVYNRLAQLPQRDRKAPCKNFFWKTFTSC

$>$ Fathead minnow (P. promelas) SST6 (DT250237.1)

MQLLASLVSLMLVLYSVRAAALLPVEERNLAQSRELSKDRKELILKLISGLLDGVDNSVLAG EIAQVPMDAEEPLESRLEERAVYNRLSQLPQRDRKAPCKNFFWKTFTSC

Other ray-finned fish

>White sturgeon (A. transmontanus) SST6 (AAL13249.1)

MQLRARLVSLMLVVYSLRVVAVLPGEERLSVHSNRELSKERKEGFLKLLSGLLDRVDSSVV LGEDVSPMDLEEPLDSRLEERAIYNRLSQLPLRARKAPCKNFFWKTFTSC

>Chinese sturgeon (A. sinensis) SST6 (FJ792688.1)

MQLRASLVSLMLVVYSLRVVAVLPGEERLSVHSNRELSKERKEGFLKLLSGLLDGVDSSVV LGEDVSPMDLEEPLDSRLEERAVYNRLSQLPQRARKAPCKN

>Spotted gar (L. oculatus) SST6 (XP_006642047.1)

MQLLASLASLMLVVYSVRAAAVLPGEERATLHTKELSKDRKELILKLISGLLDGADSAMLA GDVASVDLEEPLESRLEERAVYNRLSQLPQRDRKAPCKNFFWKTFTSC

\section{Cartilaginous fish}

>Elephant shark (C. milii) SSTc (XP_007895974.1)

MQILVALISTLFLVSGVSTTTASLDDRFNLQSSREMNQERKEMILKLLSGLLDNAAVSGQTG SEAGYPEPADGGPVMLEERSRYSQLPQRSRKVPCKNFFWKTFTSC

$>$ Whale shark (R. typus) SSTc (XP_020368528.1)

MQLLGSASXVSILLIVSSVKATAPLEDRLSLRANQELNKERKEVVLKLLSALLDSSSNQMGIE NTFPDQEDMEEMKVEDRSRFSQLPQRERKTSCKNFFWKTFTSC

$>$ Catshark $($ S. canicula) SSTc $($ JX964752) $=$ SST6

MQLLGTASLLSMLLIVSSVRATAPLEDRLSLRANQELNKERKEVILKLLSGLLDSSSNQIAIE NTFPDPEDVEEMKVEERSRFSQLPQRERKAPCKNFFWKTFTSC

\section{Other somatostatins}




\section{Lampreys}

>Japanese lamprey (Lethenteron japonicum) SSTa (KU522234)

MPAMPTTCVLLLFSLGVTALLPRPLGASPFRRPAEGSSLAGLGAWALGPGAGXGGAGLIDD RLQQRVEAVLTQELSREERALVGRLLAELAAGGAGLGGALPPLGWGPGSSSDEEGGEEGEG AGGDGGRGERALSARVRKAGCKNFFWKTFTSC

$>$ River lamprey (Lampetra fluviatilis) SSTb (KX345282)

MKISRATTTTTSTFSSTLSTSSTSSCRAVRLLFVLPMLSVVLGSVTADVRPLHVRMALERVM DGPGMENLDQDQKQFLVRLLAAIDSSADETNELNHSEDGSLPEVLVARALRAAAAPGAAG GAQLPLGNRERKAGCKNFFWKTFSSC

>Japanese lamprey (Lethenteron japonicum) SSTb (KU522235)

MKISRATTSTTSTFSSTLSTSSTTSSCRAVRLLFLLSVLSVVLGSVTADVRPLHARMALERVM DGLGMENLDQDQKLFLVRLLAAIDSSADETNELNHSEDGSLPEALVARALRAAAAPGAAG GAQLPLGNRERKAGCKNFFWKTFSSC

> Japanese lamprey (Lethenteron japonicum) SSTc (KU522236)

MKATAAICCSLALVLVATGGRRGGGLHAASIGRSAILRAPASYRQLEAHPDLQMGDQDDSQ MLLNMIVEAMRMRDAGPETDGPGEGEAARVVDGLPEFPREEVRALGPNERPFVPVVLSPRD RKANCRMFYWKTMAAC

$>$ Sea lamprey (Petromyzon marinus) SSTc (KU529940)

MKATAAICCSLALVLVASGGRRSGLHAASIGRSAILRAPASFRQLEAHPDLQMGDQDDSQIL LNMIVEAMRMRDAGPETDGPGEGEATRVVDGLPEFPREEARALVPNERPFVPGALSPRDRK ANCRMFYWKTMAAC 


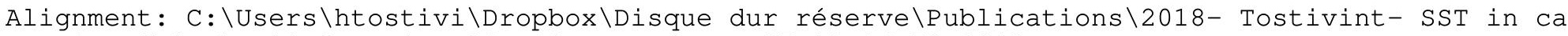
Seaview [blocks=10 fontsize=10 A4] on Wed Sep 05 10:26:59 2018

2Human

2Mouse

2Pig

2Bovine

2 Monkey

2Lizard

$2 \mathrm{Frog}$

2Coelacanth

2Lepisosteus

2Astyanax

2Pygocentrus

2Clupea

2 Ictalurus

2zebrafish

Catshark2

Whale_shark2

Elephant_shark2

Skate2

aLethenteron

bLethenteron

bLampetra

cPetromyzon

cLethenteron

3Lepisosteus

3Astyanax

3Clupea

3 Ictalurus

3Pygocentrus

3Amazon_molly

3Platyfish

3Rainbow_trout

3Freshwater_butterfly

3Grass_carp

3Goldfish

3Clown_knifefish

30range-spotted

3American_anglerfish

3Silver_arowana

3White_sucker

3Elephant_nose_fish

3zebrafish

3Stickleback

3 Takifugu

3 Tetraodon

3Medaka

3aAtlantic_salmon

3bAtlantic_salmon

Elephant_shark3

Whale_shark3

catshark3

Catshark 1

Whale_shark 1

Elephant_shark1

1 Human

1 Mouse

1Monkey

1Bovine

1Pig

1Dog

1Chicken

$1 \mathrm{Flycatcher}$

1 Turkey

1Anole_lizard

1Chinese_softshell_turtle

1 Frog

1 Lungfish

1 Coelacanth

1 Lepisosteus

1White_sturgeon

1Chinese_sturgeon

1Platyfish

1Tilapia

1Elephant_nose_fish

1 Carp

1Goldfish
1
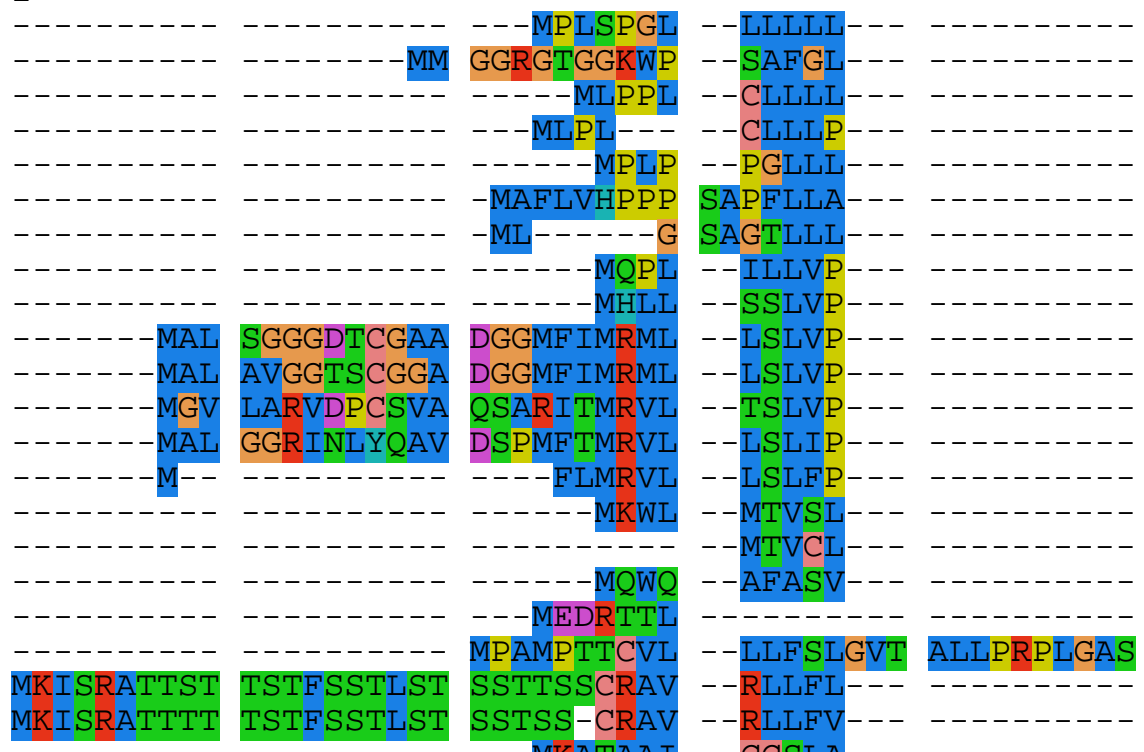

$-M E D R T T I$

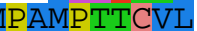

SSTTSSCRAV

SSTSS-CRAV

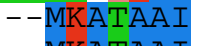

$---M K A T A A I$

MLSYRVH

-MMRF SGIH

$--M R T C V L Q$

-MRVCVLQ

MHHARCP

MHHARYP

$-M K V C R I H$

MKLCQVH

MRLCELQ

MRLCELH

MKNCQAQ

MQCIRCP

MQCIRCP

MKICQIH

MRLCELH

MKTCQVH

MRLCELQ

MQYTRCP

MQRIHSS

MPGVRS S

MQHARGP

MKF SQIH

MKVCRIH

- LLFSLGVT

ALLPRPLGAS

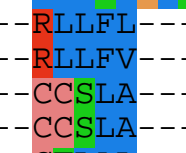

----------

$-\mathrm{CCSLA}---$

-CTLAL--- ----------

- CGLVL--- ----------

- CSLAL--- ----------

$-\mathrm{CCLTL}---\quad----------$

- CCLAL--- ---------

TILVI

AILVL--- ----------

-CILA

-CYLA

$----------$

-- TILVL--- ----------

AILAL--- ----------

- CTLVL--- ----------

-CYLAL--- ----------

L-- - - -

$--\mathrm{AILVL}-------------$

-- TILML--- ----------

AFVVLAA--- ----------

$--\mathrm{CALAL}---\quad----------$

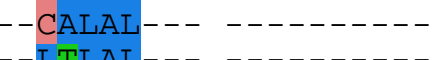

LTLAL--- ----------
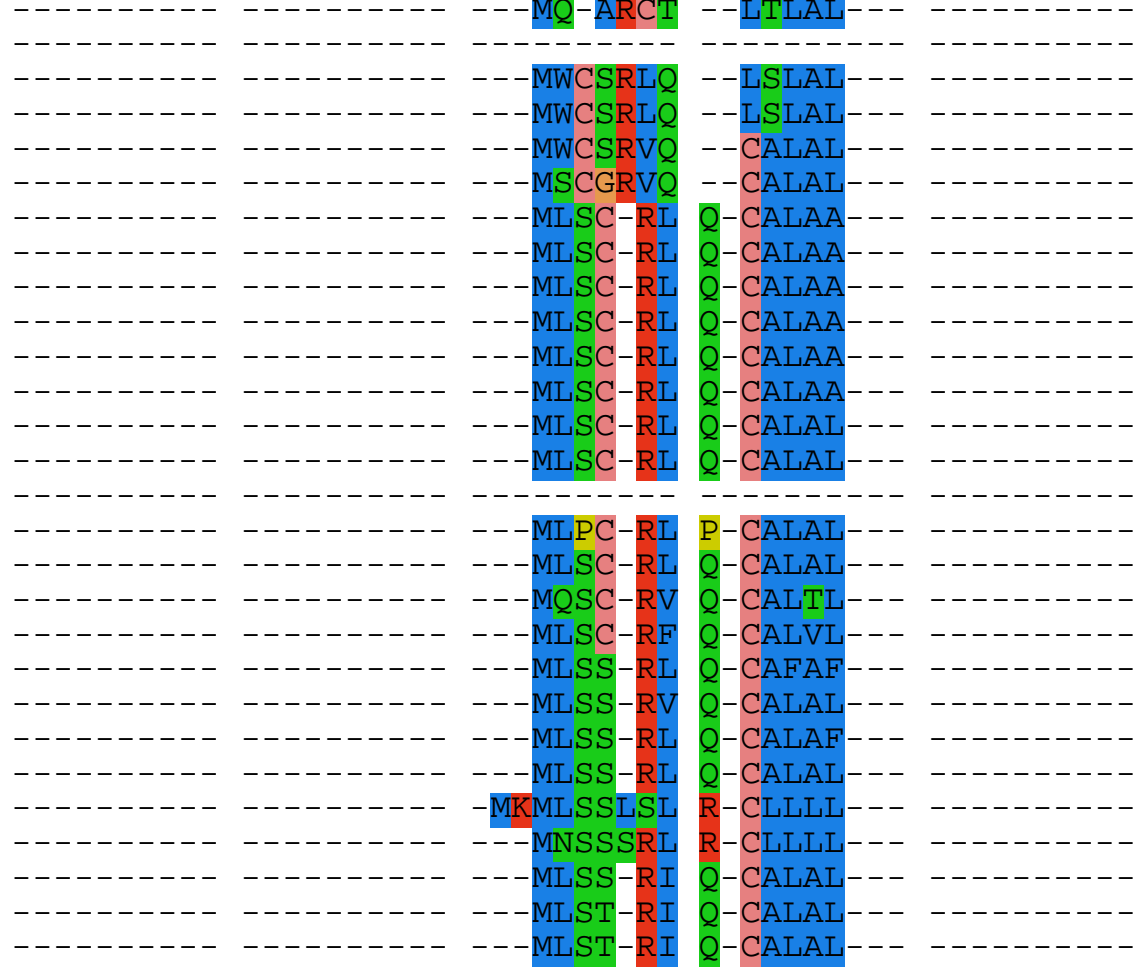
1Clown_knife_fish

1Orange-spotted_grouper

1Anglerfish

1Megalobrama

1African_cichlid_fish

$1 \mathrm{Cod}$

1Zebrafish

1 Medaka

1Stickleback

1 Takifugu

1 Tetraodon

1 Trout

1Fathead_minnow

1Killifish

1Salmon

1Pygocentrus

1 Ictalurus

1 Astyanax

1Clupea

1Amazon_molly

5 Coelacanth

5zebrafish

5Stickleback

5 Takifugu

5 Tetraodon

5 Medaka

5Sockeyess

5 Trout

5Fathead_minnow

5Amazon_molly

5Platyfish

5Tilapia

5Lepisosteus

5 Astyanax

5 Ictalurus

5Pygocentrus

Catshark5

6 Chicken

6Zebra_finch

6Duck

6Lungfish

6Coelacanth

6White_sturgeon

6Chinese_sturgeon

6Lepisosteus

6 Carp

6Goldfish

60range-spotted_grouper

6zebrafish

6Stickleback

6 Takifugu

6 Tetraodon

6Rainbow_smelt

6 Trout

6 Medaka

6 Fathead_minnow

6Astyanax

6Pygocentrus

6Clupea

6 Ictalurus

6 Amazon_molly

$6 \mathrm{Cod}$

6Platyfish

Catshark 6

Elephant_shark 6

whale_shark 6

4Lepisosteus

4 zebrafish

4 Takifugu

4 Medaka

4 Tetraodon

4Channel_catfish

4. Stickleback

4 Atlantic
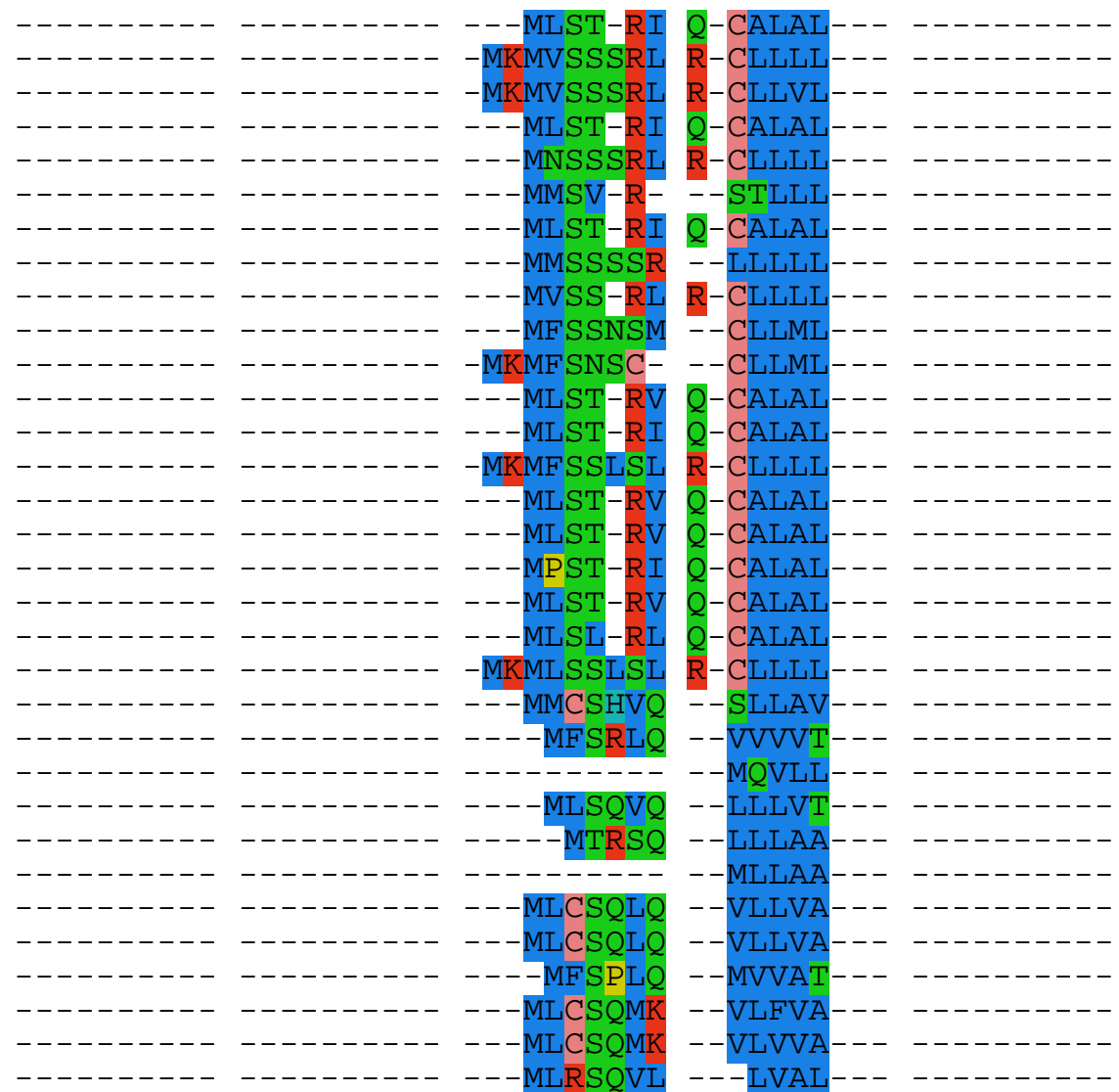

$\mathrm{R}-\mathrm{C}$

LLLLI

CLLLI

$\begin{array}{lll}-\mathrm{CLLML}--- & ---------- \\ -\mathrm{CLLML}--- & ----------\end{array}$

$\begin{array}{ll}\text { Q-CALAL--- } & ---------- \\ \text { O-CATAL-- } & ----------\end{array}$

R-

CLLLL--- ---------

-CALAL--- ----------

-CALAL--- ----------

\begin{tabular}{ll} 
QCALAL --- & ---------- \\
QCALAL --- & ---------- \\
\hline & ---------
\end{tabular}

-CLLLL--- ----------

-- VVVVT--- ----------

- MQVLL-D- ----------

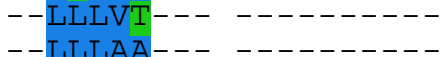

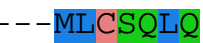

MLCSQLQ

MF SPLQ

MLC SQMK

MLCSQM

MSTG QPSLPPAPAP

MLRSQV

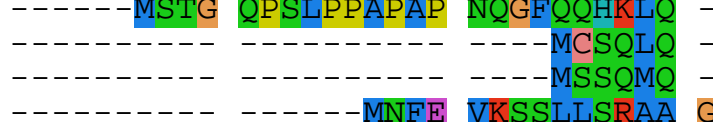

-MLLAA--- ----------

- VLLVVA--- ----------

$--M V V A T---\quad----------$

$--V L F V A-------------$

$\begin{array}{ll}- \text { VLVVA--- } & ---------- \\ ---\mathrm{LV} V \mathrm{~L}--- & ----------\end{array}$

$--\mathrm{VLLVA}---\quad----------$
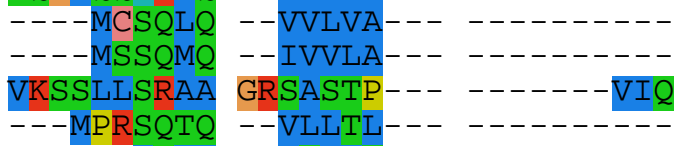

GRSASTP

VIQ

VLLTI

- ASLVS--- ----------

ASLVS--- ----------

$-A S T V S$

NMQL

MQLR

MQLP

MQLI

MQL

MQLI

MQLI

MQLI

MQLI

MQLI

MQLI

MQLI

MQLI

MQLI

$\mathrm{MQII}$

MQLI

MQLI

MQLI

MQLI

MQLM

MQLI

$-M Q L$

MQI LVAL

MQLLGSASX

MLSYRVH

MASSQLH

-MARAI

MANVI

-MARAI

MSSSPLR

-MAH II

MALTGIK

- TSLVS--- ----------

ARTVS

$-A S L V S-------------$

ASIVVS-- - - - - - - -

-SSLVS--- ----------

-VVLAA--- ----------

$--\mathrm{ASLVS}---\quad----------$

-VVLAT--- ----------

- VVLAA--- ----------

- VVLAA--- ----------

$\begin{array}{ll}- \text { VILAS }--- & ---------- \\ -- \text { VVLA }--- & ----------\end{array}$

- ASLVS--- ---------

$--\mathrm{ASLVS}-------------$

$-A S L V S$

$-\mathrm{ASLVS}------------$

$-A S L V S$

- VVLAA--- ----------

- VVLAA

- VVLAA--- - - - - - - - -

- GTASI

$-S T L F I------------$

VS I I I - - -

-CTLAL--- ----------

- LTATI

-- CILVL--- ----------

$-C V L A F$

$--\mathrm{CFLVL}---$----------

$--\mathrm{LALAL}-------------$

$-\mathrm{CVFAL}---\quad----------$

$-\mathrm{CAFVI}$ 


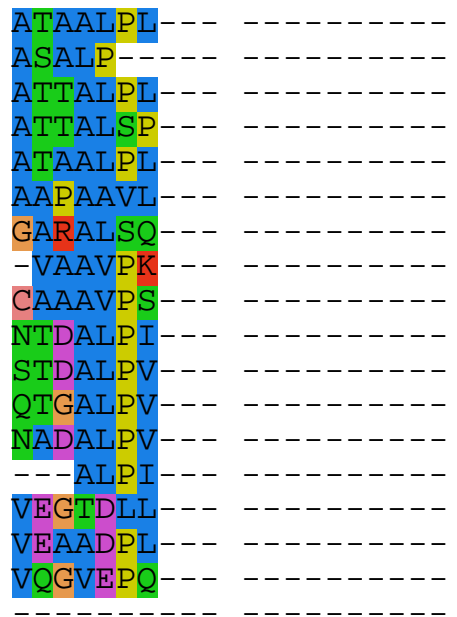

$2 \mathrm{Pig}$

2Bovine

2Monkey

2Lizard

2Frog

2Coelacanth

2Lepisosteus

2Astyanax

2Pygocentrus

2Clupea

2 Ictalurus

2 Zebrafish

Catshark2

Whale_shark2

Elephant_shark2

Skate2

aLethenteron

bLethenteron

bLampetra

cPetromyzon

cLethenteron

3Lepisosteus

3Astyanax

3Clupea

3 Ictalurus

3Pygocentrus

3Amazon_molly

3Platyfish

3Rainbow_trout

3Freshwater_butterfly

3Grass_carp

3 Goldfish

3Clown_knifefish

30range-spotted

3American_anglerfish

3Silver_arowana

3White_sucker

3Elephant_nose_fish

3Zebrafish

3Stickleback

3 Takifugu

3 Tetraodon

3Medaka

3aAtlantic_salmon

3bAtlantic salmon

Elephant_shark3

Whale shark3

catshark3

Catshark1

Whale_shark1

Elephant_shark1

1 Human

1Mouse

1Monkey

1Bovine

1Pig

$1 \mathrm{Dog}$

1Chicken

$1 \mathrm{Flycatcher}$

1 Turkey

1Anole_lizard

1Chinese_softshell_turtle 1 Frog

1 Lungfish

1Coelacanth

1 Lepisosteus

1White_sturgeon

1Chinese_sturgeon

1Platyfish

1 Tilapia

1Elephant_nose_fish

1 Carp

1Goldfish

1Clown_knife_fish

1Orange-spotted_grouper

1Anglerfish
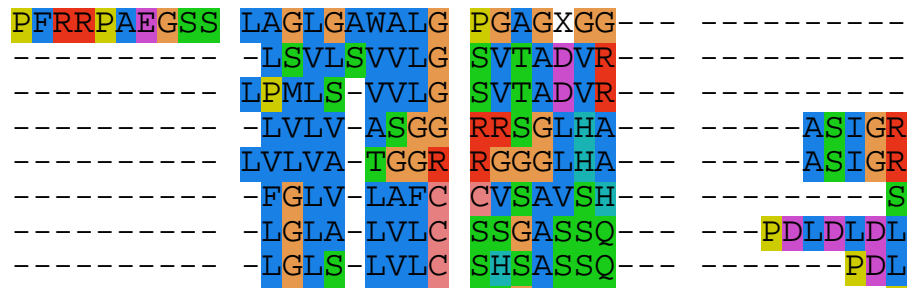

$---------$
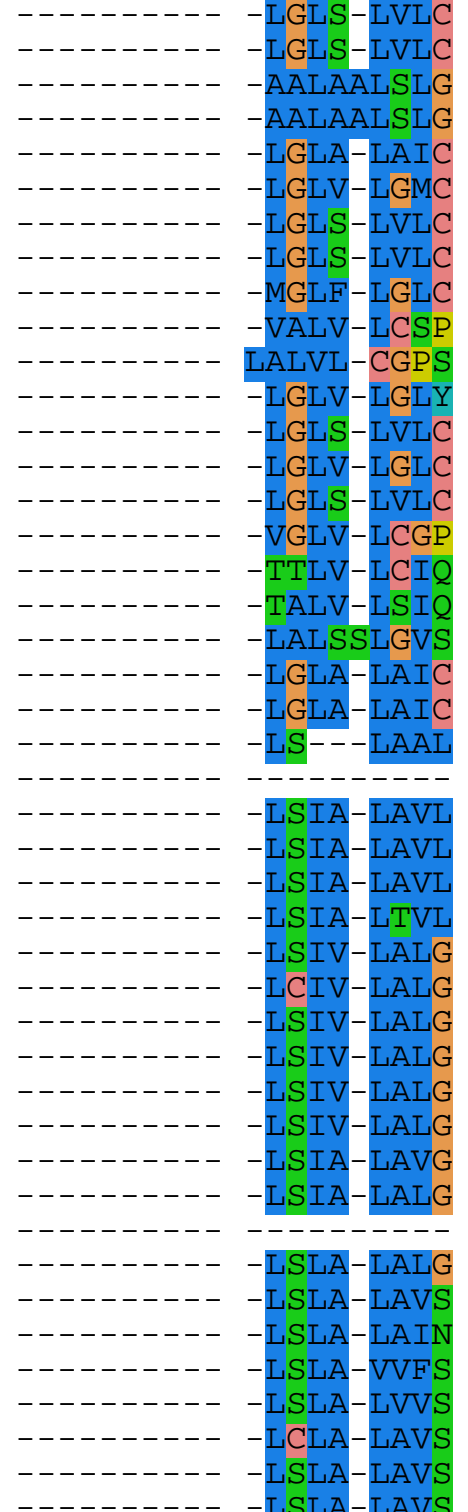

$\begin{array}{ll}\text { RRSGLHA--- } & -----A \text { AS IGR } \\ \text { RGGGLHA--- } & -----A \text { A I GR }\end{array}$

CVSAVSH -

SSGASSQ

SHSASSQ

GNGASSQ--P

VSSQADR-DQ

VSSQADR

SQGAASQ---

GSSSATQ

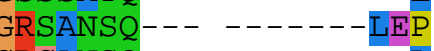

GRCANSQ

WPSDASQ

GVF SQPD

VSSQLDR--- --EQSDNQDI

CPSAASQ--- --------P

YPSAASQ--- --------P

$\begin{array}{ll}\text { GRSANSQ--- } & ---------I \\ \text { GVSSQRNRDQ } & \text { DQDQYQNQD }\end{array}$

GVSSQPD--- ---RDLNQNQ

AVSSQPD--- ---RDLDQNQ

SQSDRDQ--- ----DQFQNH

SQGAASQ--- $--------P$
SOGAASQ--- $---------P$

GLSALPT

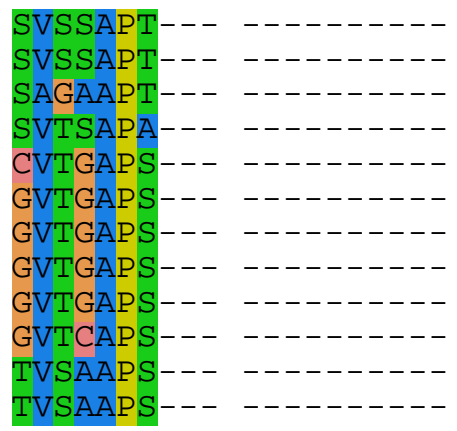

IVSAAPS

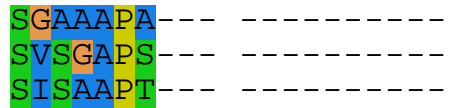

SISAAP T

KVSAAPS

NISAAPS

SVSAAPS
SVSAAP S

SVSAATS

CSSAAQR

CTSAAQR

SVSAAPS

SVSAAPT
SVSAAPT

SVYAAPS

CSSAAQR

CSFAGQR
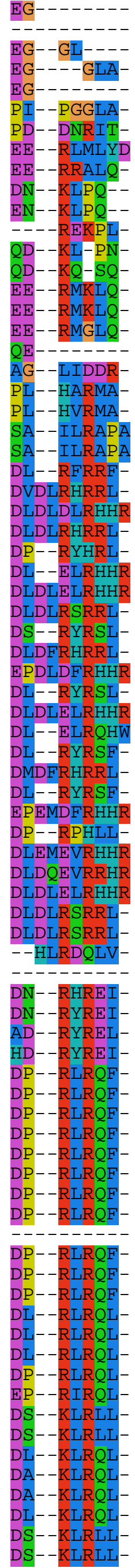
1 Megalobrama

1African cichlid fish

$1 \mathrm{Cod}$

1Zebrafish

1 Medaka

1Stickleback

1 Takifugu

1 Tetraodon

1 Trout

1Fathead_minnow

1Killifish

1Salmon

1Pygocentrus

1 Ictalurus

1Astyanax

1Clupea

1 Amazon_molly

5 Coelacanth

5Zebrafish

5Stickleback

5 Takifugu

5 Tetraodon

5 Medaka

5Sockeyess

5 Trout

5Fathead_minnow

5Amazon_molly

5Platyfish

5Tilapia

5Lepisosteus

5Astyanax

5 Ictalurus

5Pygocentrus

Catshark 5

6 Chicken

6Zebra_finch

6Duck

6Lungfish

6Coelacanth

6White_sturgeon

6Chinese_sturgeon

6Lepisosteus

6 Carp

6Goldfish

6Orange-spotted_grouper

6zebrafish

6Stickleback

6 Takifugu

6 Tetraodon

6Rainbow_smelt

6 Trout

6 Medaka

6Fathead_minnow

6Astyanax

6Pygocentrus

6Clupea

6 Ictalurus

6Amazon_molly

$6 \mathrm{Cod}$

6Platyfish

Catshark 6

Elephant_shark 6

whale_shark 6

4 Lepisosteus

4 Zebrafish

4 Takifugu

4 Medaka

4 Tetraodon

4Channel_catfish

4Stickleback

4Atlantic
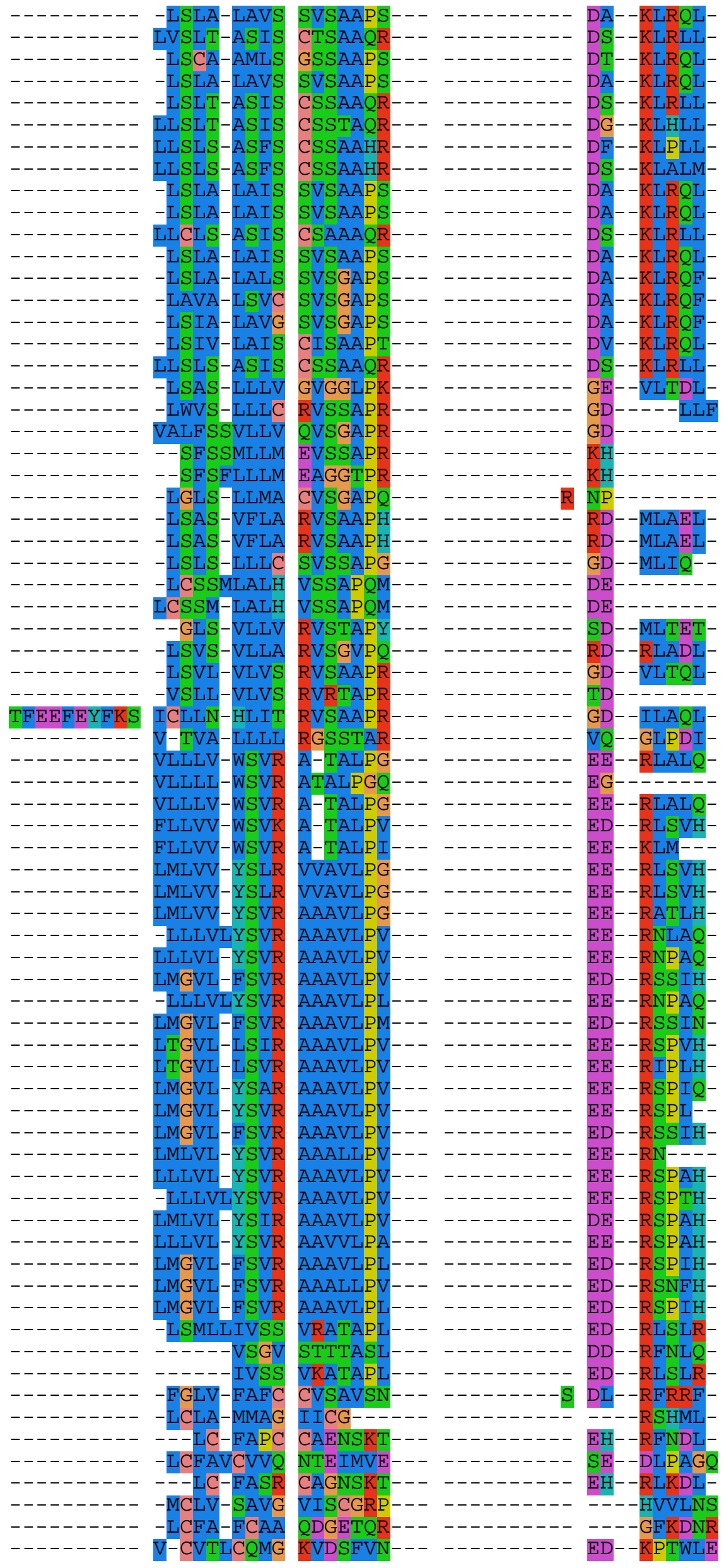

RVSAAPH-

SVSSAPG-

VSSAPOM--- - - - - - - - -

VSSAPQM

RVSTAPY

RVSGVPQ-

RVSAAPR--- ---------

RVRTAPR

RVSAAP

RGSSTAR-

A-TALPG

ATALPGQ
A-TALPG

A-TALP

A-TALPI

PG-- - --------

VVAVLPG

AAAVLPG--- ---------

AAAVLPV

AAAVLP

AAAVLP

AAAVLP

AAAVLPM

AAAVLP

AAAVLP

AAAVLP

AAAVLP

AAAVLP

AAALLP

AAAVLP

AAAVLP

AAAVLP

AAVVLP

AAAVLP

AAALLP

AAAVLP

STTTAS

VKATAPI

I ICG---- -

CAENSKT-

NTEIMVE

CAGNSKT

VISCGRP

QDGETQR

KVDSFVN

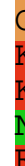

$\mathrm{GD}$
$\mathrm{KH}$
$\mathrm{KH}$
$\mathrm{NP}$

$\mathrm{H}--------$

NP- ------

RD--MLAEL-

GD-MLAEL-

$\mathrm{D}$

SD--MLTET-

RD--RLADL-

VLTQL-

ILAQL-

GLPDI-

-RLALQ-

EE--RLALQ-

ED--RLSVH-

$\mathrm{E}$

$\mathrm{SVH}-$

EE--RNLAQ-

EE--RNPAQ-

ED--RSSIH-

EE--RNPAQ-

ED--RSSIN-

EE--RSPVH-

EE--RIPLH-

EE--RSPIQ-

EE--RSPL--

ED--RSSIH-

EE-- RN----

EE--RSPTH-

DE--RSPAH-

EE--RSPAH-

ED--RSPIH-

ED--RSNFH-

ED--RSP IH-

ED--RLSLR-

DD--RFNLQ-

S

DL--RFRRF-

EH--RFNDL-

SE--DLPAGQ

EH--RLKDL-
---- HVVLNS

GFKDNR

ED--KPTWLE
EE--RATLH- 
2 Human

2Mouse

2Pig

2Bovine

2Monkey

2Lizard

2Frog

2Coelacanth

2Lepisosteus

2Astyanax

2Pygocentrus

2Clupea

2 Ictalurus

2Zebrafish

Catshark2

Whale_shark2

Elephant_shark2

Skate2

aLethenteron

bLethenteron

bLampetra

cPetromyzon

cLethenteron

3Lepisosteus

3Astyanax

3Clupea

3 Ictalurus

3Pygocentrus

3Amazon_molly

3Platyfish

3Rainbow_trout

3Freshwater_butterfly

3Grass_carp

3Goldfish

3Clown_knifefish

30range-spotted

3American_anglerfish

3Silver_arowana

3White_sucker

3Elephant_nose_fish

3Zebrafish

3Stickleback

3 Takifugu

3Tetraodon

3Medaka

3aAtlantic_salmon

3bAtlantic_salmon

Elephant_shark3

Whale_shark 3

catshark3

Catshark1

Whale_shark 1

Elephant_shark1

1 Human

1Mouse

1Monkey

1Bovine

1Pig

$1 \mathrm{Dog}$

1Chicken

1Flycatcher

1 Turkey

1Anole_lizard

1Chinese_softshell_turtle 1Frog

1 Lungfish

1Coelacanth

1 Lepisosteus

1White_sturgeon

1Chinese_sturgeon

1Platyfish

1Tilapia

1Elephant_nose_fish

1 Carp

1 Goldfish

1Clown_knife_fish

1Orange-spotted_grouper 1Anglerfish

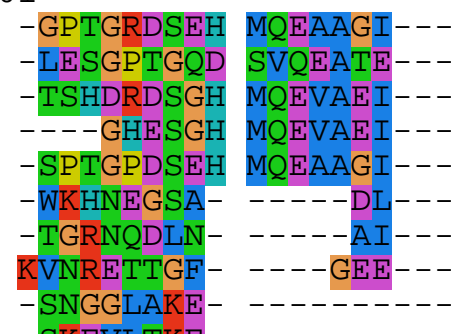

-SKEVLTKE- ----------

- SNEVLTKE- ----------
- RNESLADE-

--SNEVLTKE ---------

$\begin{array}{ll}--T N E I L S K E & ---------- \\ \text {-VNREMTKS- } & ----------\end{array}$

-VNREMTKS- ---------
-VNREVTKS- ----------
-LNREMVKD- ---------

$-\mathrm{LNREMVKD}-$
$-\mathrm{LTKN}-------------$

-LQQRVEAVL TQELSRE---
-LERVMDGLG MENLDQD---

-LERVMDGPG MENLDQD---

SFRQLEAHPD LQMGDQD---

SYRQLEAHPD LQMGDQD---

-LQKAKAAG - - LNKQDS---
-LQRARALG - - IAAQEW---

LLQRARAAG - -MSTQEW---

-LQRVQALG - -VAAQEW---

-LQRARALG - -MAAQDW---
LLQRARSAG - -LLSQDW---

LLQRARSAG - -LLSQDW---

-LQRARAAAW

-VQRARAAS- MGPQQDW---

-LQRARAIG- -QATQDW---

LLQRASATG- QATRWDF---
-LORAHSAP - -ASPODW---

-LQRAHSAP - -ASPQDW---
LLQRARSAG - -LLSQEW---

LLERARSAG - -LLSQEW---

-LQRAHAAA - -MSPQDW---

-LQRARAIG - -LATQDW---

-MQRAHTAA - -MSPQDW---

LLQRARAIG - --QAQEW---
-LQRARGAG - -LLSQDW---

LLQHVHGAG - -LLSQEW---

LLQRAHGAG - -LLSQDW---

LLQRARSAG - -LLSQDW---

-LQRARAAS - - IATQEW---

-LQRARAAG - -IATQEW---
-LQGSPA--- ----MEL---

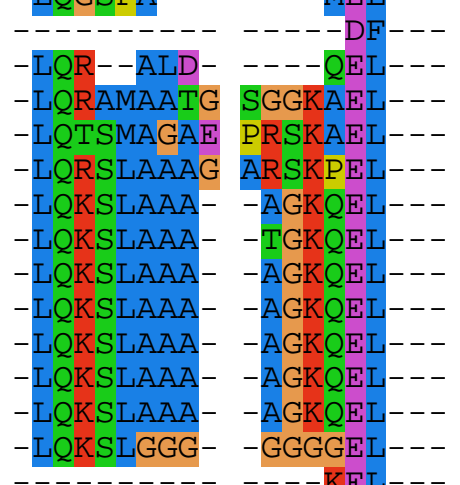

---------- ----KEL---

-LQKSLAAA - AAGKQEL---
-LQKSLAAA - -AGKQEL---

-LQKSLASA - --GKQEL---

-LQRSLAAA - -AGKQEL---

-LQKSLAAA - -AGKQEV---
-LQRSLAAS - -AGKQEL---

-LQRTLAAS - -AGKQEL---

-LQRALVAS - -AGKQDL---

-LHRTPLLS- - SKQDA---

-LHRTPLLG- - -SKQDM---

-LQRSLLAP - -ASKQDL---

-LQRSLLNP - -AGKQEL---

-LQRSLLNP - -AGKQEL---

-LQRSIIAP - -ASKQEL---

-LHRTPLLG- --SKQDM---

-LHRYPLQG- - -SKQDM---
RKSSLLT-FL AWWEEWTS-- QASAGPLI--

GRSGLLT-FL AWWHEWAS-- QASSSTPV--

KKNSLLT-FL AWWHEWMS-- QAREVPFV--

KTNSLLT-FL AWWYEWAS-- QARAVPFV--

RKSSLLT-FL AWWFEWTS-- QASAGPLI--

GRDELLA-FL SGLVDRASRR HGMPLSK---

QQDLLLK-LL SGWTD--SRE SN--LVE---

RKQFLSN-ML DALIGL---- NTLENDTP--

RKDVLLK-VL SGLLVS---- NLLRKEQS--

QKDLLIK-IM SDLAELNMTL KDLGALDL--

QKDLLIK-IM SDLAELNMTV KDLGALDL--

ERILLLK-IL AGLTEKNLTE ENLTGHDL--

QKDFMTQ-FL ANLAELNMT I KDLETMDH--

EDGL--K-MI TYVAELNLTS KELADLDA--

AGLLETDD-- NLLENGFA--

SGLLDPDD-- NLLENDFT--

AGLLDVDD-- NLLESVIA--

AGLLDVDD-- HLGESGYT--

AELAAGGAGL GGALPPLGWG

AAIDSSADET NELNHSED-G

AAIDSSADET NELNHSED-G

VEAMRMRDAG PETDGPGEGE

VEAMRMRDAG PETDGPGEGE

SQLVQSDS-- EALENEMS--

SQLTLPESEF QESEISTL--

SQLSLPEEET QDREVSTV--

AQLTMPESEF QENDVSTL--

SQLTLPESEF KESEITSL--

AQMSRSAV-- EAQQDDPE--

AQMSRSEV-- EAQQDGPE--

-RWRTFYPNC PCLRPRKV--

SLLAATEA-- DMPFGDMS--

SLLSMPEM-- EMRENDIS--

SLLSIPEM-- EMREKGLS--

ARLPPPEG-- EFTPVEDV--

AQMSLPEA-- DTQREAEV--

AQMSLPEA-- DVQREAED--

SRLAPAQG-- EVPQGAVS--

SQLSLPEI-- EARENGVS--

SRLAPPED-- DTPQSEMS--

SLLSMPEM-- QMRESDLS--

AQMSLPEA-- DSQLGAEV--

AQMSMPEG-- TGQRDTEV--

AQMSLPEG-- TGQRDTEV--

ARMPDPEA-- EAEREAEV--

SKLSLPDV-- EAQKS-----

SQLSWPEA-- QESEVSPA--

QAVADAESKP QSEEAAE---

HVVADADKDA VEVNASQV--

PLVTDVDTEA GTLDVSNA--

SELENAEN-- EALETDAM--

AELANAEN-- EVLDGEDL--

AELANAEN -- EALEAEDM--

SEPNQTEN-- DALEPEDL--

SEPNQTEN-- DALEPEDL--

SEPNQTEN-- DALEPEDL--

SEPNQTEI-- DALEPEDL--

SEPNQTEN-- DALEPEDL--

SEPNQTEN-- DALEPEDL--

SEPSQTEN-- EALESEDL--

SEPSQTEN-- EALESEDL--

SEPSQTEN-- EALESEDL--

SEPGQAEN-- EALEPEEL--

AEPSQPEN-- EALEPEDL--

SEPSQTDN-- EALESDDL--

SELAOSEN-- DALDSSDL--

AELAQAEN-- EALESDDF--

SELAOSEN-- EALESDDL--

SELAQSEN-- DALASDEL--

SELAQSEN-- DALASDEL--

SDLLQMEN-- EALEEDGF--

SDLLQVEN-- EALEEENF--

SEMVRVEN-- EALEPDDL--

SDLVQAEN-- EALEPEDL--

SELVQAEN-- EALEPEDL--

SELAQVEN-- EALESDDL--

SDLLQVEN-- EALEEENF--

SDLLQGEN-- EALEEENF-- 
1Megalobrama

1African cichlid fish

$1 \mathrm{Cod}$

1Zebrafish

1 Medaka

1Stickleback

1 Takifugu

1 Tetraodon

1 Trout

1Fathead_minnow

1Killifish

1Salmon

1Pygocentrus

1 Ictalurus

1 Astyanax

1Clupea

1 Amazon_molly

5 Coelacanth

5Zebrafish

5.Stickleback

5 Takifugu

5Tetraodon

5 Medaka

5Sockeyess

5 Trout

5Fathead_minnow

5Amazon_molly

5Platyfish

5Tilapia

5Lepisosteus

5Astyanax

5 Ictalurus

5Pygocentrus

Catshark 5

6 Chicken

6zebra_finch

6Duck

6Lungfish

6 Coelacanth

6White_sturgeon

6Chinese_sturgeon

6Lepisosteus

$6 \mathrm{Carp}$

6Goldfish

6Orange-spotted_grouper

6zebrafish

6Stickleback

6 Takifugu

6Tetraodon

6Rainbow_smelt

6 Trout

6 Medaka

6Fathead_minnow

6Astyanax

6Pygocentrus

6Clupea

6 Ictalurus

6Amazon_molly

$6 \mathrm{Cod}$

6Platyfish

Catshark 6

Elephant_shark 6

whale_shark 6

4 Lepisosteus

4 Zebrafish

4 Takifugu

4 Medaka

4 Tetraodon

4Channel_catfish

4Stickleback

4Atlantic

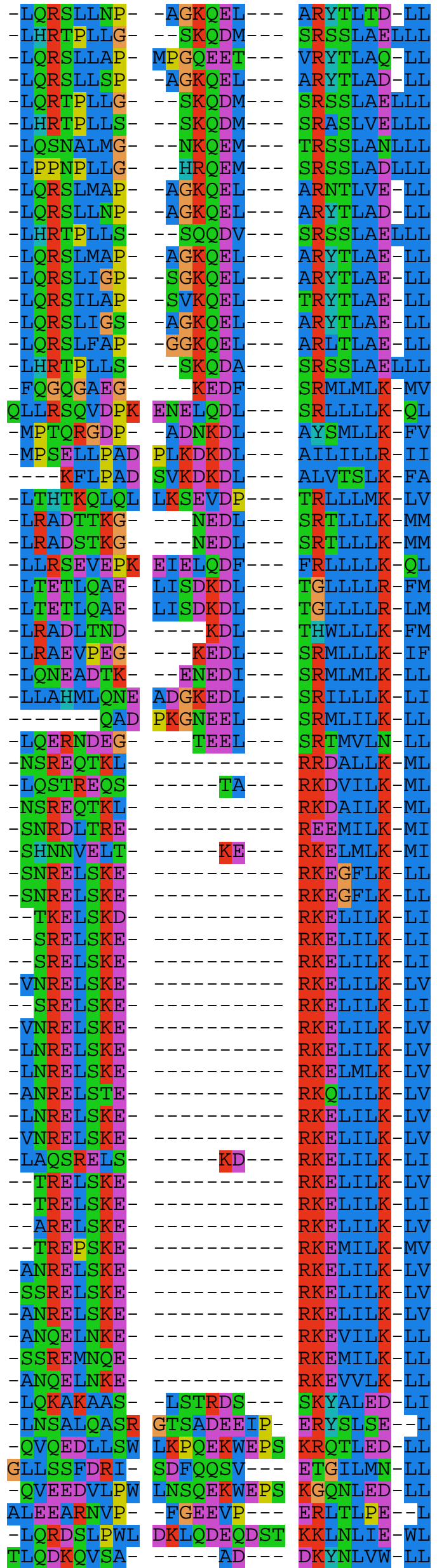

SDLVQAEN-- EVLEPEDL--

SDLLOVEN-- EALEEENF--

SELAQAEN-- EALGTDDQ--

SDLVOAEN-- EALEPEDL--

SDLLQMEN-- EVLEEDGF--

SDLLQVQN-- EALEEENL--

SDLLQVGN-- EALEENLA--

SDLLQVEN-- QALDENFP--

SELAHVEN-- EAIELDDM--

SELVOAEN-- EALEPEDL--

SDLLQMEN - - EALEEDGF--

SELAOAEN-- EAIESDDL--

SELAQAEN-- EALEAEDM--

AELAQAEN-- EVLDSDEV--

SELAQAEN-- EALEDDVP--

SELAQAEN-- EALESEDM--

SDLLOMEN -- EALEEDGF--

SNLLKSEN-- DVLVPSAE--

SESVTPEEK - DALDSIDE--

SELTAARGGE TLLVPEQE--

SEVMAAER-- EMLLLPQE--

FEGTAAQR-- EMLLLLQE--

LELVALRR - - QEMLQELE--

SDLMSTAVGE NEVLPDLE--

SDLMSAAVGE NEVLPDLE--

SESAAPE--- EKQPRESI--

SELMASRG-- EEMLREKO--

SELTASRGEE

AELMAAR---

SDLLRAEN-- ---EIPPG--

SELEVAGEN- EVLSGADV--

SELKIPDEN - DTLSDDDM--

SDLEIPGD-N EVLSGTDV--

SELLHAGD-

AGLLESSD-

AGLLGSVD-

AGLLESVD--

- - -VASPDI--

SGLLDGSD-- NSLFGGEV--

AGLLEGID-- SPPMGGEV--

SGLLDRVDS - SVVLGEDV--

SGLLDGVDS - SVVLGEDV--

SGLLDGAD-- SAMLAGDV--

SGLLDGVD-- NNVLAGEIAP

SGLLDGVD-- NSVLDGEIAP

SGLLDGALDT NMLPGEAA--

SGLLDGVD-- NSVLAGEIAP

SGLLDGAADA NMLPGEAA--

SGLLDGALDT NMLPMETV--

SGLLDGALDT NMLPMEAV--

SGLLDGASDS NMLPGDVS--

SGLLDGATDT NMLPGEGV--

SGLLDGSLDT NMLPGEAA--

SGLLDGVD-- NSVLAGEIAQ

SGLLDGVD-- NSVLGGDL--

TGLLDGVD-- SSVLAGDL--

SGLLDGVE-- SNMLPGDE--

SSLLDGVD-- NNMLAGDL--

SGLLDGALDT NMLPGEAA--

SGLLEGSPDS NMLPGELS--

SGLLDGALDT NMLPGEAA--

SGLLDSSS-- NQIAIENT--

SGLLDNAAVS GQTGSEAG--

SALLDSSS-- NQMGIENT--

SQIAQSDN-- EALENEMS--

EWLLSNSD-- PAVFQPDSSS

YKLTNYKSEN RILPQQPG--

YKLSRPDSAI TLQQVRDS--

YKLTNYRSEN RIVSQQPS--

QWMLSN---- NELTPVQV--

YSFYKFDNG-

NIVKGPTD--

YKLSQSHHD- NTFKINFPPH 
2 Human

2Mouse

2Pig

2Bovine

2Monkey

2Lizard

2Frog

2Coelacanth

Lepisosteus

2Astyanax

2Pygocentrus

2Clupea

2 Ictalurus

2Zebrafish

Catshark2

Whale_shark2

Elephant_shark2

Skate2

aLethenteron

bLethenteron

bLampetra

cPetromyzon

cLethenteron

3Lepisosteus

3Astyanax

3Clupea

3 Ictalurus

3Pygocentrus

3Amazon_molly

3Platyfish

3Rainbow_trout

3Freshwater_butterfly

3Grass_carp

3 Goldfish

3Clown_knifefish

30range-spotted

3American_anglerfish

3 Silver arowana

3White_sucker

3Elephant_nose_fish

3zebrafish

3Stickleback

3 Takifugu

3Tetraodon

3Medaka

3aAtlantic_salmon

3bAtlantic salmon

Elephant_shark3

Whale shark 3

catshark3

Catshark1

Whale_shark 1

Elephant shark1

1 Human

1Mouse

1Monkey

1Bovine

1Pig

$1 \mathrm{Dog}$

1Chicken

$1 \mathrm{Flycatcher}$

1 Turkey

IAnole lizard

1Chinese_softshell_turtle

1 Frog

1 Lungfish

1Coelacanth

1 Lepisosteus

1White_sturgeon

1 Chinese_sturgeon

1Platyfish

1 Tilapia

1Elephant_nose_fish

1 Carp

1 Goldfish

$1 \mathrm{Clown} k n i f e$ fish

1Orange-spotted_grouper

1 Anglerfish

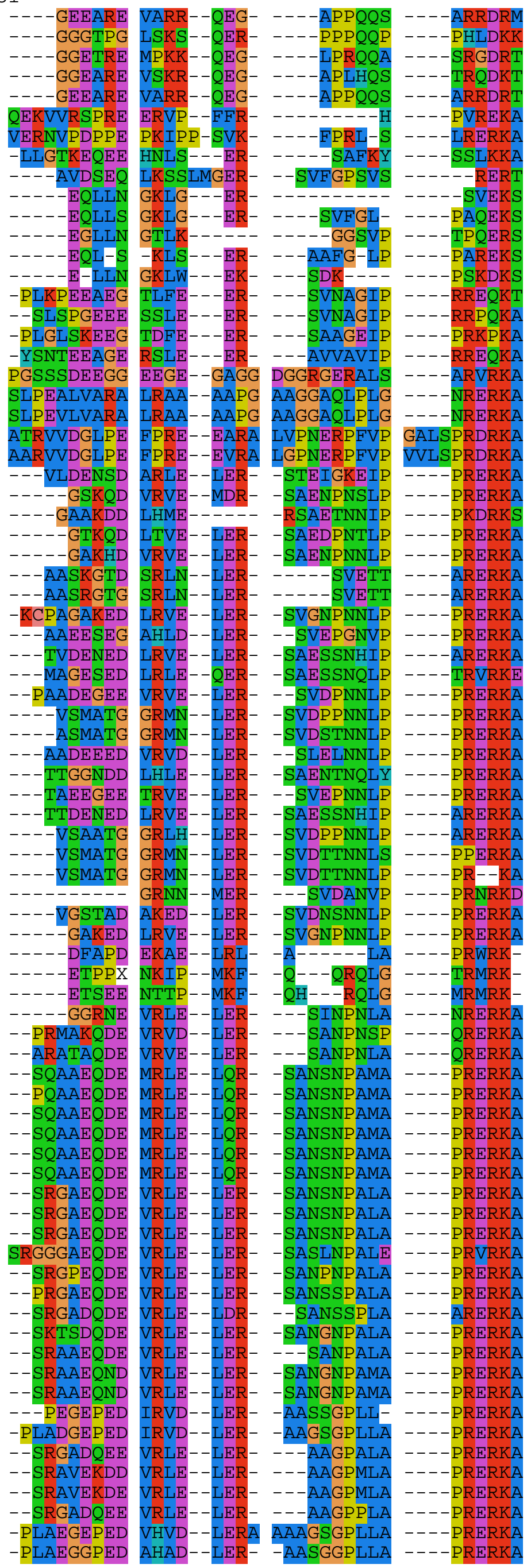

CRNFFWKTE

PCKNEFWKT

PCGNFFWKTE

CKNEFWKTE

PCKNFFWKTF

PCKNFFWKTF

PCKNEFWKTE

PCRNEFWKTE

CKNFFWKTF

PCTNEFWKTE

CKNFEWKTF

PCKNFFWKTF

QCN-FFWKTF

PCKLFFWKTF

PCKLFFWKTF

PCKLFFWKTF

GCKLFFWKTF

PCKLFFWKTF

GCKNFFWKTF

GCKNFFWKTF

GCKNFFWKTF

NCRMFYWKTM

NCRMFYWKTM

GCRNFYWKTF

GCKNFYWKGF

GCKNFFWKGR

GCKNFYWKGF

GCKNFYWKGF

GCKNFYWKGL

GCKNFYWKGI

GCKNFYWKGF

GCKNFYWKGF

GCKNFYWKGF

GCKNFYWKGF

GCKNFYWKGF

GCKNFYWKGF

GCKNFYWKGF

GCKNFYWKGF

GCKNFYWKGF

GCKNFYWKGF

GCKNFYWKGF

GCKNFYWKGF

GCKNFYWKGP

GCKNFYWKGF

GCKNFYWKGF

GCKNFYWKGF

GCKNFYWKGF

CKNFFWKTY

NCKNEFWKTY

NCKNFFWKTY

GCKNFFWKTF

GCKNFFWKTF

GCKSFFWKTF

GCKNEFWKTF

GCKNFFWKTF

GCKNFFWKTF

GCKNFFWKTF

GCKNFFWKTF

GCKNFFWKTF

GCKNFFWKTE

GCKNFFWKTF

GCKNFFWKTF

GCKNFFWKTF

GCKNFFWKTF

GCKNFFWKTF

GCKNFFWKTF

GCKNFFWKTF

GCKNFFWKTF

GCKNFFWKTF

GCKNFFWKTF

SCKNFFWKTF

GCKNFFWKTF

GCKNFFWKTF

GCKNFFWKTF

GCKNFFWKTF

GCKNFFWKTF

GCKNFFWKTF

GCKNEFWKTF 
1 Megalobrama

1African cichlid fish

$1 \mathrm{Cod}$

1Zebrafish

1 Medaka

1Stickleback

1 Takifugu

1 Tetraodon

1 Trout

1Fathead_minnow

1Killifish

1Salmon

1Pygocentrus

1 Ictalurus

1 Astyanax

1Clupea

1 Amazon_molly

5 Coelacanth

5Zebrafish

5Stickleback

5 Takifugu

5Tetraodon

5 Medaka

5Sockeyess

5 Trout

5Fathead_minnow

5Amazon_molly

5Platyfish

5Tilapia

5Lepisosteus

5Astyanax

5 Ictalurus

5Pygocentrus

Catshark 5

6 Chicken

6Zebra_finch

6Duck

6Lungfish

6 Coelacanth

6White_sturgeon

6Chinese_sturgeon

6Lepisosteus

$6 \mathrm{Carp}$

6Goldfish

60range-spotted_grouper

6zebrafish

6Stickleback

6 Takifugu

6 Tetraodon

6Rainbow_smelt

6 Trout

6 Medaka

6Fathead_minnow

6Astyanax

6Pygocentrus

6Clupea

6 Ictalurus

6Amazon_molly

$6 \mathrm{Cod}$

6Platyfish

Catshark 6

Elephant_shark 6

whale_shark 6

4 Lepisosteus

4 Zebrafish

4 Takifugu

4 Medaka

4 Tetraodon

4Channel_catfish

4Stickleback

4 Atlantic
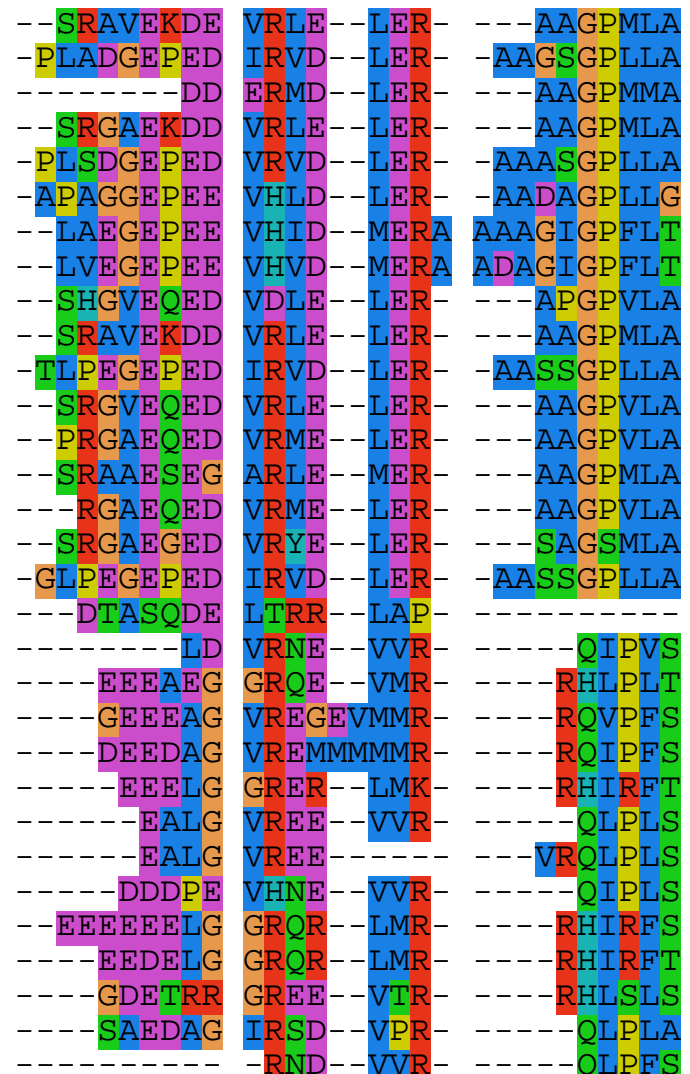

$----P R E R K A$
$----P R E R K A$
$----P R E R K A$
$----P R E R K A$

GCKNFFWKTF

CKNFFWKTF

GCKNFFWKTF

GCKNFFWKTF

----PRDRKA GCKNFFWKTF

----PRERKA GCKNEFWKTF

----PRERKA GCKNFFWKTF

----PRERKA GCKNEFWKTF

----PRERKA GCKNFFWKTF

----PRERKA GCKNEFWKTF

----PRERKA GCKNFFWKTF

----PRERKA GCKNFFWKTF

----PRERKA GCKNFFWKTF

----PRERKA GCKNEFWKTF

----PRERKA GCKNFFWKTF

----PRERKA GCKNFFWKTF

----PRERKA GCKNFFWKTF

SRERKA GCKNFFWKTF

QRERKA GCRNFYWKTF

QRERKA GCRNFFWKTF

QRDRKA GCRNFFWKTF

QRERKA GCRNFFWKSY

QRERKA GCRNEFWKTF

GCRNFFWKTF

GCRNFFWKTF

GCRNFYWKTF

GCRNFFWKTF

GCRNFFWKTF

GCRNFFWKTF

GCRNFFWKTF

GCRNFFWKTF

GCRNFFWKTF

GCRNFFWKTF

GCKNFFWKTF

PCKNEFWKTF

PCKNFFWKTF

PCKNFFWKTF

PCKNEFWKTF

PCKNFFWKTF

PCKNFFWKTF

PCKNFFWKTF

PCKNFFWKTF

PCKNEFWKTF

PCKNEFWKTF

PCKNFFWKTF

PCKNEFWKTF

PCKNEFWKTE

PCKNFFWKTF

PCKNFFWKTF

PCKNEFWKTF

PCKNFFWKTF

$\begin{array}{ll}----Q R D R K A & \text { PCKNFFWKTF } \\ ----Q R D R K A & \text { PCKNFFWKTF }\end{array}$

----QRDRKA PCKNFFWKTF

----QRDRKA PCKNFFWKTF

----QRDRKA PCKNFFWKTF

----QRDRKA PCKNFFWKTF

----QRDRKA PCKNFFWKTF

----QRDRKA PCKNFFWKTF

----QRDRKA PCKNFFWKTF

----QRERKA PCKNFFWKTF

----QRSRKV PCKNFFWKTF

----QRERKT SCKNFFWKTF

----PRERKA GCRNYYWKT

----KEERKT GCKNYFWKSR

GCRMFFWKSW

PCRVFYWKSW

GCRIFFWKSW

-CMNYFWKSR

GCRVFFWKSW

GCKVFFWKSW 
2 Human

2Mouse

2Pig

2Bovine

2Monkey

2Lizard

2Frog

2Colacanth

2Lepisosteus

2Astyanax

2Pygocentrus

2Clupea

2 Ictalurus

2Zebrafish

Catshark2

Whale_shark2

Elephant_shark2

Skate2

aLethenteron

bLethenteron

bLampetra

cPetromyzon

cLethenteron

3Lepisosteus

3Astyanax

3 Clupea

3 Ictalurus

3Pygocentrus

3Amazon_molly

3Platyfish

3Rainbow_trout

3Freshwater_butterfly

3Grass_carp

3Goldfish

3Clown_knifefish

30range-spotted

3American_anglerfish

3Silver_arowana

3White_sucker

3Elephant_nose_fish

3zebrafish

3Stickleback

3Takifugu

3 Tetraodon

3 Medaka

3aAtlantic_salmon

3bAtlantic_salmon

Elephant_shark3

Whale_shark3

catshark 3

Catshark 1

Whale_shark 1

Elephant_shark1

1 Human

1 Mouse

1Monkey

1Bovine

1Pig

$1 \mathrm{D} \circ \mathrm{g}$

1 Chicken

$1 \mathrm{Flycatcher}$

1 Turkey

1Anole_lizard

1Chinese_softshell_turtle 1Frog

1 Lungfish

1 Coelacanth

1 Lepisosteus

1White_sturgeon

1Chinese_sturgeon

1Platyfish

1 Tilapia

1Elephant_nose_fish

1 Carp

1 Goldfish

1Clown_knife_fish

1Orange-spotted_grouper

1Anglerfish 
1 Megalobrama

1African cichlid fish

$1 \mathrm{cod}$

1Zebrafish

1 Medaka

1Stickleback

1 Takifugu

1 Tetraodon

1 Trout

1Fathead_minnow

1Killifish

1Salmon

1Pygocentrus

1 Ictalurus

1Astyanax

1Clupea

1 Amazon_molly

5 Coelacanth

5Zebrafish

5Stickleback

5 Takifugu

5 Tetraodon

5 Medaka

5Sockeyess

5 Trout

5Fathead_minnow

5Amazon_molly

5Platyfish

5Tilapia

5Lepisosteus

5Astyanax

5 Ictalurus

5Pygocentrus

Catshark 5

6 Chicken

6Zebra_finch

6Duck

6Lungfish

6Coelacanth

6White_sturgeon

6Chinese_sturgeon

6Lepisosteus

6 Carp

6Goldfish

6Orange-spotted_grouper

6Zebrafish

6Stickleback

6 Takifugu

6 Tetraodon

6Rainbow_smelt

6 Trout

6 Medaka

6Fathead_minnow

6Astyanax

6Pygocentrus

6Clupea

6 Ictalurus

6Amazon_molly

$6 \mathrm{Cod}$

6Platyfish

Catshark 6

Elephant_shark 6

whale_shark 6

4 Lepisosteus

4 Zebrafish

4 Takifugu

4 Medaka

4 Tetraodon

4Channel_catfish

4Stickleback

4Atlantic 


\section{Figure S3}

\section{A. Catshark SST1 (SSTa)}

gctgtccacagagccagtgctgaaaccaccgccaatttcaaagcgtttgtaaa atgtggtgcagccgccttcagttgagcctggctctcctctccatcgccctggcagtactg

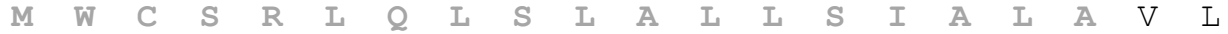
agtgtcagctcagcacccacagacaacaggtatcgcgaaatcctgcagagagccatggca $\begin{array}{llllllllllllllllllllll}S & V & S & S & A & P & T & D & N & R & Y & R & E & I & L & Q & R & A & M & A\end{array}$ gccacaggaagcggagggaaagcggagttgacgaaatattccctcgcccaactgctctcc $\begin{array}{llllllllllllllllllll}A & T & G & S & G & G & K & A & E & L & T & K & Y & S & L & A & Q & L & L & S\end{array}$ gaactggaaaacgcggagaatgaagctctggaaacggatgcaatgggcggacgcaacgag $\begin{array}{llllllllllllllllllll}E & \mathrm{~L} & \mathrm{E} & \mathrm{N} & \mathrm{A} & \mathrm{E} & \mathrm{N} & \mathrm{E} & \mathrm{A} & \mathrm{L} & \mathrm{E} & \mathrm{T} & \mathrm{D} & \mathrm{A} & \mathrm{M} & \mathrm{G} & \mathrm{G} & \mathrm{R} & \mathrm{N} & \mathrm{E}\end{array}$ gtgcggctcgaactggagagatcgataaatcccaacctggcgaacagggagcgaaaagcg $\begin{array}{llllllllllllllllllll}V & R & L & E & L & E & R & S & I & N & P & N & L & A & N & R & E & \mathbb{R} & \mathbf{K} & \mathbf{A}\end{array}$ ggatgtaagaacttcttttggaaaccttcacatcctgttaactctccgaattctccata

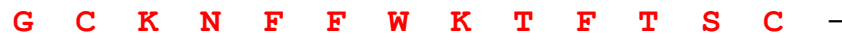

gcaacccacacactcagaatgtggcattcagataactaaataalcatgtggtgaatgat tctatttgaattatgtaagagaagacaattggtttgaattgtccaatagcaggccagttt gaatggtgactggatagagtcgctatttttcattaatctgttgcaataaatctctgttt gaaattg

\section{B. Catshark SST2 (SSTd)}

tttagcattttgtccaggagtgctcgaagattcagc atgaagtggctaatgacagtgagcctgatgtctctgctgtaccttctgagagtcgagggt

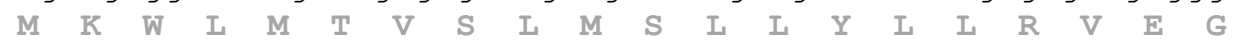
actgacctactggaagaaagaatgaaacttcaggtaaacagagaaatgacaaaaagcagg $\begin{array}{llllllllllllllllllll}T & D & L & L & E & E & R & M & K & L & Q & V & N & R & E & M & T & K & S & R\end{array}$ aagaatctgattgtaaagctactggctggcttgttggagactgatgataatctcttggag $\begin{array}{llllllllllllllllllll}K & N & L & I & V & K & L & L & A & G & L & L & E & T & D & D & N & L & L & E\end{array}$ aatggctttgcacctttgaaaccagaagaggcagaagggactttgttcgaagagaggtcg $\begin{array}{llllllllllllllllllll}N & G & F & A & P & L & K & P & E & E & A & E & G & T & L & F & E & E & R & S\end{array}$ gttaacgctggaattccacgcagggagcaaaaaccccatgcaaactgttcttctggaaa $\begin{array}{llllllllllllllllllll}\mathrm{V} & \mathrm{N} & \mathrm{A} & \mathrm{G} & \mathrm{I} & \mathrm{P} & \mathbf{R} & \mathbf{R} & \mathrm{E} & \mathrm{Q} & \mathbf{K} & \mathrm{T} & \mathrm{P} & \mathrm{C} & \mathrm{K} & \mathrm{L} & \mathbf{F} & \mathbf{F} & \mathbf{W} & \mathbf{K}\end{array}$ acctttcccactgttaataaccgtgtagcggattttctaaagcagtgggagtatgaaa T $\mathrm{F} \quad \mathrm{S}$ H $\mathrm{C}-$

gaaaatgggtgtaaaggcttaactttaaactatcgaaaatacactgcaatcatgtttt tctctatcccaatatttaattcaaatttacaggaaatgcttgcaataag ttaacctga aagttacaccttttaagacatgtgaaattattgtgttttaaatgagatagcaatccagtt aactttctcagtttgtttctatcatgcctgttatttccctgtttttccagcattgtttt cttctctcagttcctcgcttcctttggcatgttagccccctctcttcccacagaatgct cctgggattccgtactctccccctcgctttaccttccctcttcgctagacgaatgtt ctgaaagattgcacctgcccctgacacagccggctgggacttctggaagcacttgaattt tgccagacacaatttgggatattttctgagttaaagcctatatatatgttgacagtgacc tttcctcttgatcgtgagatctgagcatattgtaatggtgttaattgttttgaccagtct atctgcatacaatgtacatacttatctcgaaatatcctgtgagtaatgctaacaataaa agaca

\section{Catshark SST3 (SSTe)}

ggagagacagtgctgaaacgccctctccactttccgtcggcttcttcgccttgtgcagag atgtggtgcagccgccttcagttgagcctggctctcctctccatcgccctggcagtactg $\begin{array}{llllllllllllllllllll}M & W & C & S & R & L & Q & L & S & L & A & L & L & S & I & A & L & A & V & L\end{array}$ agtgtcagctcagcccccacagacaacaggcatcgcgaaatcctgcagagagccttggat $\begin{array}{llllllllllllllllllllllllllllll}S & V & S & S & A & P & T & D & N & R & H & R & E & I & L & Q & R & A & L & D\end{array}$ caggaacttctgaaggacacaatgatgaaactgttgcctttggttactgatgtagacact $\begin{array}{llllllllllllllllllll}Q & E & L & L & K & D & T & M & M & K & L & L & P & L & V & T & D & V & D & T\end{array}$ gaggccggaacactggatgtttccaatgctgagacttcagaagaaaacacaacaccgatg $\begin{array}{llllllllllllllllllll}E & A & G & T & L & D & V & S & N & A & E & T & S & E & E & N & T & T & P & M\end{array}$ aaattccagcatcgtcagttgggaatgagaatgcggaaaaactgcaagaacttctttgg 
aaaactacactttatgctaatccactgtacccagtgagcccttctcactggaccetgct

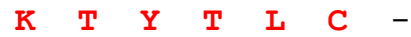

gtaccccac

\section{Catshark SST5 (SSTb)}

tcgaccctcgcgtccggagcagttacacagagaagctcgcctctcgcccacactgcccga cgagacagtttgccaatcgttcccatctcccactccgcagtaagaaatcagagctcgag atgccgagatcgcagactcaagtcctcctcactctggtcactgtcgctctgctgttactg

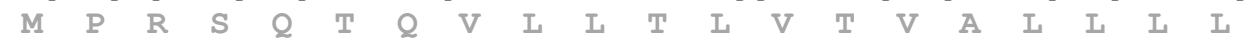
cggggatcgagtacagccagagtgcaggggctgcccgatatcttacaggagaggaacgac

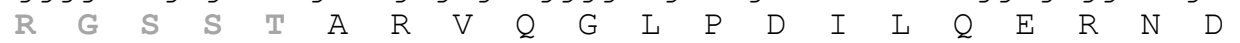
gaggggactgaggagctgtccaggaccatggtgctgaacctcctctccgagctgctccat $\begin{array}{llllllllllllllllllll}E & G & T & E & E & L & S & R & T & M & V & L & N & L & L & S & E & L & L & H\end{array}$ gctggggacaaggggttgcccagcaccgagagacgggagcttccccaggctgagatcgtc $\begin{array}{llllllllllllllllllll}A & G & D & K & G & L & P & S & T & E & R & R & E & L & P & Q & A & E & I & V\end{array}$ cgccggtggaactcaccgcgggaccgcaaagcaggctgcaagaacttcttttggaaaacc $\begin{array}{llllllllllllllllllll}R & R & W & N & S & P & R & D & R & K & A & G & C & K & N & F & F & W & K & T\end{array}$ ttcacctcttgttaacgtgcggagagagagagggaaagagaaagtgagccggcgggggga F $\quad \mathrm{T} \quad \mathrm{S}$ C -

gagatataagagaattcctccctctcccaaaacccatcttcagctcctgaagggaggca aaaaaaatcaattgcctctaacttgtagattttaagattcacaaatcccaaccacctcc gtccctcgaaagattgcctccccctttgggctgcctctccgctgaggatttctctgtgtc tttatccaaggtgtttgtaalgattgacttgtttgctttgttctctgagcatggacttg ctctcacatcataaatatctttattctcagcaaaaaaaaaaaa

\section{E. Catshark SST6 (SSTc)}

tcgaccctcgcgtccgccaaggtcgaggcaca ttgagaagatctcggccaggctccgcttcattccaacttcacaagatcgagagtgtcagc atgcagttgctgggaacggcgagcctcctgtccatgctgctcatcgtgtccagtgtgagg $M$ \& I I G T A S I L S M I I I V S S V R gccacggcacctttggaagatcggctgagtctgcgggcaaatcaggagctgaacaaagaa $\begin{array}{llllllllllllllllllll}A & T & A & P & L & E & D & R & L & S & L & R & A & N & Q & E & L & N & K & E\end{array}$ aggaaagaggtcattttgaagctgttatccggactgcttgactccagcagcaatcaaatc $\begin{array}{llllllllllllllllllll}R & K & E & V & I & L & K & L & L & S & G & L & L & D & S & S & S & N & Q & I\end{array}$ gcgattgagaacactttcccggatccagaggatgtggaggagatgaaagtggaggagagg $\begin{array}{llllllllllllllllllll}A & I & E & N & T & F & P & D & P & E & D & V & E & E & M & K & V & E & E & R\end{array}$ tcccgcttcagccagttaccgcagagagagcgcaaggctccctgcaaaaacttcttttgg $\begin{array}{llllllllllllllllllll}S & R & F & S & Q & L & P & Q & R & E & R & \mathbb{R} & \mathbf{A} & P & C & K & \mathbf{N} & \mathbf{F} & \mathbf{F} & \mathbf{W}\end{array}$ aaaacttcacctcctgctgacgacagacaatgccacctggctgccaactaaagccgccg $\begin{array}{lllllll}K & T & F & T & S & C\end{array}$

agaaaggaactgatttagcactgtacataagctgcttttattttattcatctgtgaggg gtggtggaggtggggggggggttaatattttctgtcgtgcacaagaaaaataaaaagagc atgattgtgtgatttcaccgcaacctgtatgtatttatttctttcccagtaatgttgtc gcgctcaacctgccagagcaataaagtggatccgagataaaaaaaaaa 


\section{Figure S4}

\section{A. Catshark SST1 $=$ SSa (scaffold122178)}

AACAGAAGTAAAATGATACCTGGTCCCAGTGTGTAAATCTGTAACAGCCCCATTTCTGTGGCATTTCAAT GAAAAGCTAGTTTAAACCTGACGAATACAGTGGAGTTATTGAGATGAACACATTGCAGATTGGCACATGT GGGCCACAAAGAGAACACGCTGTTAAAATCACAAACTACACACAATTATTCAGATGGCAGCGAGTCTTTC CCCAGGAAACATTCCTTTATTCTGTCTCACTCAGTCTCTAACTTTGTACATTCAGTTCACTGGGATTACC CAACCCATTCCCCACTGATCCAGTGTGTTCAATGCTCAAGGAAGATTGAGATTTATGGATGAGGGTGTTT ACTGGGGATTGATGTTCACTTTTTCCACGGATTTTCTAGTTGAAGACTTGGCTGCATTCATTTTGTGATC GACACGAACCAATACAATCGTAGGGCCAGCTGCCAACACGCGATAGGGTGTCCACATTGAGACTCCATGG ATCTATCTCAATCTTCCTCATGGGTAATGGGTTGGTTGAGGATGTGACTGGGTTGTGCGTAAATACTCGT CATCTCCTGGGACCCTCCTCCCAACGTGTGAGCTGGTGTGAAGGCGGCTTTAAAAGGAGCTTCCCCGATT GCAGGGACAGAAGGAGCGGAGCTGTCCACAGAGCCAGTGCTGAAACCACCGCCAATTTCAAAGCGTTTGG AAACTGCTCTCCTTCTCGCTGTCTCTCCCGTTGATCTTCGCCTTGTGCAGA ATGTGGTGCAGCCGCCTT

$$
\begin{array}{lllllllll}
M & W & C & S & R & L
\end{array}
$$

CAGTTGAGCCTGGCTCTCCTCTCCATCGCCCTGGCAGTACTGAGTGTCAGCTCAGCACCCACAGACAACA

$\begin{array}{lllllllllllllllllllllll}2 & \mathrm{~L} & \mathrm{~S} & \mathrm{~L} & \mathrm{~A} & \mathrm{~L} & \mathrm{~L} & \mathrm{~S} & \mathrm{I} & \mathrm{A} & \mathrm{L} & \mathrm{A} & \mathrm{V} & \mathrm{L} & \mathrm{S} & \mathrm{V} & \mathrm{S} & \mathrm{S} & \mathrm{A} & \mathrm{P} & \mathrm{T} & \mathrm{D} & \mathrm{N}\end{array}$
GGTATCGCGAAATCCTGCAGAGAGCCATGGCAGCCACAGGAAGCGGAGGGAAAGCGGTAAGGACCCAGCA $\begin{array}{lllllllllllllllllll}R & Y & R & E & I & L & Q & R & A & M & A & A & T & G & S & G & G & K & A\end{array}$

AAAAGTCACATTCAATAGAAATGGTAGTTTGTGTTCATCCCGTTCACCTCGAATAGAAACAGGGACTGGG AAATAACTGGTTCAATTCTGATCTAGTGAGAAGACCCACTTTTAACAGGACTATAATATTGAGAGTGAAT TGGCTTTGTTTAGTGTTGGGGATCATTTCATTGGTTTAGAAAGGTTTCTGAGCGCGTTGATCCAAATTAC TGGATTTCTCGTGCATCGAGTAAAATCTCGTCCTCTCCAATCCATAGCCACTCTGCACAAAGAGTAACAG ACAAGAAGTAGACATCGTTCCACAATCCCAGAGAACTTACTGCTCTTCTCTCTTCCCAAACTGCCTCTCC CATCTTAACCCCAACCCTCCTCTCTCCCCAAATCCCGTCTCCCATCCCAACCCCAACCCTCCTCTCTCCC CAAATCCCCTCTCCTGTCCCAACCCCAACCCTCCTCTCTCCCCAAGCTCCCAAACCCCCCTCTCCCATCC

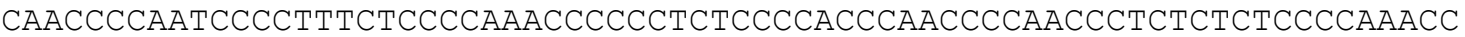
СCTCTCTCCCCACCCAACCCCCCACCCCAACCCCCTCTCTTCCCACCCTGGGACTTCTGACCAGGCTCAT TTGGTCCTGTTTATCATTTGAAGACGAGCACCTGAGTCTCTCTCAGTCACCTCCAATAACCATCTAGAAT GGGGCAGCTGAATCAAATCTTGCAACCAAGTCATTCAGTTTGAAAGAAATATTCATTACAATTATGTTTT CAATTGCCTTCATGTTGAAAAGAAACTCCGAGCACGGGGATTGGTTCCTTCCAGAGATTTTCTCGCTCTT TCCAATGTCCACCTCCCTTTGAAGGGTTCAGAATTGTGGTGGATGGAATGGAGTTCGGGGTGGAGTGGGA GGGGGTTTGGGCAGTTCAGGTCTCTATTTGCGAATTGGGATCTTCCCGGCCGGTTGCAATGGAGAAATAT GAAGATAAATGAAGATAATTTCACAAAGTTGAATTTAATTCATTGTAGATTTATTCGCGGGAGTTTGCAA CTTTCGTTTGCAAAACAAACGTAAATATAGCGTCAATTGGCGGAAATTTCTCCCCTAATCTATTTCCTGT TGTTCTCAGGAGTTGACGAAATATTCCCTCGCCCAACTGCTCTCCGAACTGGAAAACGCGGAGAATGAAG $\begin{array}{lllllllllllllllllll}E & L & T & K & Y & S & L & A & Q & L & L & S & E & L & E & N & A & E & N\end{array}$ CTCTGGAAACGGATGCAATGGGCGGACGCAACGAGGTGCGGCTCGAACCGGAGAGATCGAGAAATCCCAA $\begin{array}{lllllllllllllllllllllllll}\mathrm{E} & \mathrm{A} & \mathrm{L} & \mathrm{E} & \mathrm{T} & \mathrm{D} & \mathrm{A} & \mathrm{M} & \mathrm{G} & \mathrm{G} & \mathrm{R} & \mathrm{N} & \mathrm{E} & \mathrm{V} & \mathrm{R} & \mathrm{L} & \mathrm{E} & \mathrm{P} & \mathrm{E} & \mathrm{R} & \mathrm{S} & \mathrm{N} & \mathrm{P} & \mathrm{N}\end{array}$ CCTGCCGAACAGGGAGCGAAAAGCGGGATGTAAGAACTTATTTTGGAAAACCTTCACATCCTGTTAACTC $\begin{array}{lllllllllllllllllllll}\mathrm{L} & \mathrm{P} & \mathrm{N} & \mathrm{R} & \mathrm{E} & \mathbf{R} & \mathbf{K} & \mathbf{A} & \mathrm{G} & \mathbf{C} & \mathbf{K} & \mathbf{N} & \mathrm{L} & \mathbf{F} & \mathbf{W} & \mathbf{K} & \mathbf{T} & \mathbf{F} & \mathbf{T} & \mathbf{S} & \mathbf{C}\end{array}$ TCCGAATTCTCCATAGCAACCCACACACTCAGAATGTGGCATTCAGATAACTAAATAAATCATGTGGTGA ATGATTCTATTTGAATTATGTAAGAGAAGACAATTGGTTTGAATTGTCCAATAGCAGGCCAGTTTGAATG GTGACTGGATAGAGTCGCTATTTTTCATTAATCTGTTGCAATAAAATCTCTGTTTGAAAGTGGACCATTT GTTGGATTTTGTTCTCTGGGAGATGGGGCAATATAACAAGGGACAGAATTAACTCGGCTAGTTTTCAACA CCAGTCTGTGTCCAAAGGTACAGAGCGAGGGGAGTCATTTTCCACAGTTGTGACTTTGTAAATCAGCTCT GGGTGGAATCGCTCTGGGTCACTGTCTCTGATCTGGGAAATGTGTTGCTGGAAGTTCCTCCGCGTATTCA AACTCAGAGGAGACAATGAATGGACGGATATTAAAGGGGGTTCTGCTCTGTCCAATGCAACCGTGCTCTG TGAGTGCTGTAATCTGCAGGAGAGCAAACTATTCACCTGCATCACACTGGTAAAAGTCTAACCCTGGTCA ATGAAAGCATCAATCCACAAATCACAAACCTGCTTCCAGCCTTCCCTTGTAAAGCCGGTTTCCATTCTAA CCTAGCGGCACGGTAGCACAGTGGTTAGCACTGCTACTTCACAGCGGCAGGGTCCGGGGTTCGATTCCCA CTTGGGTCACTGTCCATGCGGAGTCTGCACGTTCTCCCCGTGTCTGCGTGGGTTTCCTCCGGGTGCTCCG GTTTCCTCCCACAAGTCCCGAAAGACGTGCTGTTGGGTAATTTGGACATTCGGAATTCTCCCTCTCTGTT CCCGGACAGGCACCAGAATGTGGCGACTAGGGGCTTTTCACAGTGGAAATGTCAGTGGAGGTTCTGTACA ATACCAATGTTCCGTACAGGTGTTGAATTGTGTTCTACACAGGAAATTGCCTTCCATCATCCCCTATTAT GTCTAGAAACATAGAAAATAGGTGCAGGAGGAGGTCATTTGGCCCTTTGAGCCTGTTCTGCCATTCATTA TGATCATGGCTGATCATCCAGCTCAATAGCCTTCTCCAGCGTTCCCCCATATCCTTTGATCTCCTTCACC ATAAGTGCTATATCTAACTGCTTCTTGAAAATATATTGAGAGGGATTCTCCATCCTGCCACACCAGGAGC TGGATTCTCTGCCGTCGCGATTCTCTATTTTGCCAGAAGCACGGGGGTTTCCCAACAGCGTAGGGTGGCC CCACAAGGAGAAACCCCGTTGACCAGCTGGCGAAACGGAGAATCCCGATGGCATATGGTGGGACAGAGAA 
ACCCGCCCCAGATTTCTGATGCGGCATGTCGTCGGGATTCTCCATTTCGCCGACTGGTCAATGGGGTTTC CCATCATGGGGCAGCCCCATGTCGTCGGAAAACCCTTGGGCTGGGGGTAAAATGGAAAATCATGATGGC...

\section{B. Catshark SST2 \\ (scaffold 83887)}

...САСтСтTCTCAAAAAGCAGTGGACGCAGTCTTAAATATGTTCAAGACAGAGGTAGATAGATTCTTGAA AgGTAATGgGgTGCAAGGTTGTTGGGGGTAGGCGGGAGATTACAGTCCGATGGGCCATGATCTTATTGAA TGGCAGATCAGGCTCGAAGGGCCGAGTGGCCTACACGTGCTCTTAGTTCCTATGTCTGTAACATCCGACA CTTTGGAGAAGAATATTTTTATTGTGTCAGGAATCCAGCCCTCCCAGAATTTTCCAGGGTGCTAATAAT CAGTTTTTACTTAGATTTCTAGTCAACCTACAGGTTTTAGATTTTAGTTTTCTTATACTGCATTGGAGGG TCAGTTGTTGAACAGCATCCATCCAATTCCACTTTTATAACTTCTAAAAATATAATTTTGTTTAAATGGC TGGAACATTCTTGGATTGGAGGCTATAAAGCTCTTTAATGGCTGCTTCATTCAAATTGAACTGCAATTTT AACTGTCCACATTACAGATCCTTCATCTACTTGCCCATAAATTTACAGAACGTAAAAGTGTAATATAATC AGTCAGGTATGTTCTTCCTGAATTAATATTCCTCCATTTACGATAACGCAAACCTGTTACTCCTTATATA CATATTGTTTCAGGCCTGTATTGGGTTAATGATGCACACTGGCTATTAGGCTTTGATTGTATCATTAAT TTTGTGCGTCAGAGTTGCTTCTGCTGTGCTATAAAAGAGCTGGAAACACTTTCGGAAAAATAGATTGAGG AGTTCACAATACATTTAGCATTTTGTCCAGGAGTGCTCGAAGATTCAGCATGAAGTGGCTAATGACAGTG $\begin{array}{llllllll}M & K & W & \mathrm{~L} & \mathrm{M} & \mathrm{T} & \mathrm{V}\end{array}$ AGCCTGATGTCTCTGCTGTACCTTCTGAGAGTCGAGGGTACTGACCTACTGGAAGAAAGAATGAAACTTC $\begin{array}{lllllllllllllllllllllll}S & L & M & S & L & L & Y & L & L & R & V & E & G & T & D & L & L & E & E & R & M & K & L\end{array}$ AGGTAAACAGAGTGAGTAGAAAGATCATTACTAAATGCACAGAGGTGGCGGCAGTGTGGTGAAAGGGGTG Q $\quad$ V $\quad N \quad R$

GGAGGGACGAAATCCACAGCCATAAGGAGAGAATTGACGTAAAAATATTGGGAAAATTATGTAGAGAGAT GAGAGACAATGGGATTGTTCTCTCTAATGCTAGGAGATTAAGGGAAGACTTAATAGAGGTGTTCAAAATA ATGAGAAAGCGTTTCCACAGGCAGATGGGCCGGTGACCACGAGGAGAACATTTTAAACTAATTGATAAAA GAGCAAGAGGGAGCGATGAGGAAAATTGTTTTTTACATCGTCAATCATTATAATCTGAAACGTGTTGCCT GAAAGTTGATGGAAGCAGATTCAATAGTAACTTTTCAAAAGGACTTAGATATATACTTGAAGGTTGGAAA CTTGCAGAACTATCAGGAAGAGGGGAGGGAGCACTATAAATTCTGCACTGTAAATTCTATGATTACGTTA AGGCAGGAAAATGGAAGTAATTGAGTAGCTCTTTACAAAGGGCTGGTATAGATGTGATGGGATGAATGGC CTTTTTTGTGTAAAGTTCCATGAAAGCCAACATCAGCAATAATCAGACTTTCAGATTTCCAGCATCCAC ACTATTTTGCTTTAATCAGCATTAATATATCATCAAGGGATCTGGTAGTGGGTAGTTGTCTTACTGGACA AGATTAATAATCACGAGAATTTGAGTTCAAAAACCACCATGGCAGTTTGAGAACTCGAATTTGGTTTGAA AATAAAATCTGGAAACAAGAAACTGGTTTCAGTCAAAGACGTAAACTGTCGGATTGTCATAAACCCTCAA CTGGTTCGTCAATACCTTTTTTTAATATAAGTTTAGAGTTCCCAATTAATTTTTTTCCAATTAAGGGGCA ATTTAGCGTGGCCAATCCACCAACCCTGCACATCTTTTGGGTTGTGGGGGTGAGACCCACGCAGACACGG GGAGAATGTGCAAACTCCACACGGACAGTGACCCGGGGCTGGGATCGAACCTGGGGCCTTGGCGCTGTGA GGCAGCAGTGCTAACCCACTGCGCCACCGTGCTGCCCTGGCCAATACCTTCTAAGGAAGGAAACTGATCT GCCAGGTATCCTGGGCCATAGCAATCTGTTTGGCTGTTAACAGATTTCTTAGTGACCTAACGAGCCACAC AGGTTTAACCAGGATGGCAATAATGTGCCAGTCGGACTTGGAATTCTGTGAAAAGATCAGAAAACCCGGC GCAGACTGTTCTTCGGGAAAAATTGCTGATTAAAGTTTGGTGGATTTACTTTAAACTAGTGGAACATTAT TGAAAGGACCTGTGGACCTGAACAAGCTCGACACTCATTTTTGCCTTTAATCTAATGTCCTTCAGGGAAT GAAATCTGCCGTCCTTACCTGGTCTGGCCTACATGTGACTCCAGATCCACAGCAATGTGGTTGGACTCTT AACTGCCCCCTCAAGGGCAATTAGATATGGGCAATAAATGCTGCCCAGCCTGCGACGCCCATGTCCCTTG AGCGAATAAAAATAAATCAGGCCAAGTACAAAAATATATTGAGGGAACAGAAAGATTTAGGCTTAGGGAT GTTCGAGTTGGCAAGATATGCAGAGGGTAGCAACGAGTAAACTGTAACAAAACTGGGTCAATGGTGGGAA TAATGGTTGGAAATAAAGTGTAAAAGACAACTAAAAGAGCTGAGCTAGATCTGAGTGAGTGATTCATGTT TGGCATCAACAAACAAGTGCCTTGCAATGAAATGAAAACGAAAGCTTTCTATTCTCTGTTTATCCACAA GAAATGACAAAAAGCAGGAAGAATCTGATTGTAAAGCTACTGGCTGGCTTGTTGGAGACTGATGATAATC $\begin{array}{lllllllllllllllllllllll}E & M & T & K & S & R & K & N & L & I & V & K & L & L & A & G & L & L & E & T & D & D & L\end{array}$ TCTTGGAGAATGGCTTTGCACCTTTGAAACCGGAAGAGGCAGAAGGGACTTTGTTCGAAGAGAGGTCGGT $\begin{array}{lllllllllllllllllllllllll}\mathrm{D} & \mathrm{L} & \mathrm{L} & \mathrm{E} & \mathrm{N} & \mathrm{G} & \mathrm{F} & \mathrm{A} & \mathrm{P} & \mathrm{L} & \mathrm{K} & \mathrm{P} & \mathrm{E} & \mathrm{E} & \mathrm{A} & \mathrm{E} & \mathrm{G} & \mathrm{T} & \mathrm{L} & \mathrm{F} & \mathrm{E} & \mathrm{E} & \mathrm{R} & \mathrm{S}\end{array}$ TAACGCTGGAATTCCACGCAGGGAGCAAAAAACCCCATGCAAACTGTTCTTCTGGAAAACCTTTTCCCAC $\begin{array}{lllllllllllllllllllllll}\mathrm{V} & \mathrm{N} & \mathrm{A} & \mathrm{G} & \mathrm{I} & \mathrm{P} & \mathrm{R} & \mathrm{R} & \mathrm{E} & \mathrm{Q} & \mathrm{K} & \mathrm{T} & \mathrm{P} & \mathrm{C} & \mathrm{K} & \mathrm{L} & \mathrm{F} & \mathrm{F} & \mathrm{W} & \mathrm{K} & \mathrm{T} & \mathrm{F} & \mathrm{S}\end{array}$ TGTTAATAACCGTGTAGCGGATTTTCTAAAGCAGTGGGAGTATGAAAGAAAAATGGGTGTAAAGGCTTA $\mathrm{H} \mathrm{C}-$

ACTTTAAAACTCGAAAATACACTGCAATCATGTTTTTCTCTAGCCCAAATATTTAATTCAAATTTACAGG AAATGCTTGCAATAAAGTTAACCTGAAAGTTACACCTTTTAAGACATGTGAAATTATTGTGTTTTAAATG AGATAGCAATCCAGTTAAACTTTCTCAGTTTGTTTCTATCATGCCTGTTATTTCCCTGTTTTTCCAGCAT TGTTTTCTTCTCTCAGTTCCTCGCTTTCCTTTGGCATGTTAGCCCCCTCTCTTCCCACAGAATGCTCCTG GGATTCCGTACTCTTCCCCCTCGCTTTACCTTTCCCTCTTTCCCTAGACGAATGTTTCTGAAAGATTGCA CCTGCCCCTGACACAGCCGGCTGGGACTTCTGGAAGCACTTGAATTTTGCCAGACACAATTTGGGATATT 
TTCTGAGTTAAAGCCTATATATATGTTGACAGTGACCTTTCCTCTTGATCGTGAGATCTGAGCATATTGT AATGGTGTTAATTGTTTTGACCAGTCTATCTGCATACAATGTACATACTTATCTCGAAATATCCTGTGA... 


\section{Catshark SST3 \\ (scaffold121269)}

TTAGATTCCTGGCTTGGGTCACTGTCTGTGCGGAGTCTGCACGTTCTCCCCGTGTGTGCGTGGGTTTCCT CCGGGTGCTCCGGTTTCCTCCCACAGTCCAAAGATGTGCAGGTTAGGTGGATTGGCCATGCTAAATTGTC CTCAGTGTCCAAATTCCCTTAGTGTTGGGTGGGGTTACTGAGTTATGGGGATAAGGTGGAGGTGTTGAC CTTNNNNNNNNNNNNNNNNNNNNNNNNNNNNNNNNNNNNNNNNNNNNNNNNNNNNNNNNNNNNNNNNNNN NNNNNNNNNNNNNNNNNNNNNNNNNNNNNNNNNNNNNNNNNNNNNNNNNNNNNNNNNNNNNNNNNNNNNN NNNNNNNNNNNNNNNNNNNNNNNNNNNNNNNNNNNNNNNGTGGAGGCAGTTTTCTCGCAAAGCAGGATAG TATAAAAGTAATAATATTTAAAGCAGAGCAGTCTTGACTGAGGACAAGGAAGGGGCTGAAGCACACCAGG TGAACCAGTTTTTTGAAGATATTCAGCCGAATCTGAAGGGGCTCTAATAGGAGTGTAAATATTTTCTGTG AAGCAATTTTTACTCTGTGCTATGCTGATTGTAAGATGGATTAAGAGCTGTATGTTTATTTCCTTGTTTG TTCATGGGGAATTGAGTAGTTGTCTTTAAGGGGTAATTGTAAACTGTTTTCCTCGGATGTGAAGTTAAAG AGATAATATTGTATTCATAATAAGGTTTGTTTGAGAAATACCAAACCCCTTTTATTTCACGCAATCGTT CCTGAAGTGAGTCATTCTTTCTGCATAGCTGTACAACAAACTAAAATATTAGGGTTTTGGTCCAGTATCC TGTTGTGGTCTGGTCTGGGATCACAATATGCAATACATCTCAAATTACTACATTCATCTCAGGTCATTGA TGAAGCAGCTGAAGATGGTTGGACTGAGGACACTACCCTGAGGAACTCCTGCAGTGATGGCCTGGAGCTG ACATGATTGACCTCCAACCACCACAACCATCTCCCTTTGTGCCGGGTATGACTCCAACCAGCGGAGAGTT TCCСCCCTGATTCCCATTGACTCTGTTTTGCTCGGGTGCTCTAATGTATTACAATCCTGGATCAGACCGC AACATTTGCTAAGATACCGAACAGAAACGACAATATTTTATTTTAATTTTGTAAGACTGTGAgGAAAgGA TACTTCACTCCAGGAGTGATTGCACGAAGAATTAGGGATTTGGTTTGTTAAAACAAACTTTATTACTAAC AGTAATAATAACTGTGACATCATATAAACATATAGCTTTCAATTACCAGTTAAATAATGCTTATCAGTAC AATGACACAATAACCCTCAACTGCTATTTTTATTTCCACTCAAACAACACCCATCTCAAACCAAATCCAC TTTTAAACACAATGAGCAGTCTCAGGAATCCATGCTGTAAAGAAACATCCTGGATAAACAAACTTTGATA AAGCAATATTCTTCAGCTTAGGCTCGGTGTATTGGNNNNNNNNNNNNCTTAGTGTCCAAAAATGTTAAGT GGGGGTTAGTGGGTTACAGAGATAGGGTAGATATGTGGGCTTCAGTCGGGTGCTCTTTGTAAGGGCTGGT GTAGACTTGATGGGCCGAATAACCTCCATCTGCACGGTAAATTCTATGGTTATATGGTTCTATTCTGTGG TCTCAACTTACTGTTTGAACTCCCTCTCCTGTTCATAGGCAAATCATTAATGCATTGCCTTGAGATCAGC TGCTTCTGGTCTGTCCCCAGTCAGATATTTAACTGAACTGTTTGGAAAGTTTCTCCTAGTCTGTCTACAG GCACATCTTTAACTGAACTGTATTGAGAGCAGCTGTTTTATAAAATCCCCACATGGCAGAAGGAAGCCAT TCAGCCCATTGCGTTCGCGACCCATCGAAAGACCACCCTACCTTGAACAATTCCCCCACCCTATTCCTCC AAGCCCAACCTGAGGGCAATTGATCTTGATCAATCCATCAATCTGCACATGTTTGGACTGTGGAAGGAAA CTGGAGGAAACCCATGCAAGCATGGGGAGAATGTGCAAACTTCACACAGACAGTGACCAGAGGTTGGAAT CAAACTCGGGCCCCTGCCGCTGTGAGGCAGCAGTGTTAACTACTGTACCACCATGCTTCCCTGACTCTGT GAGGCAGTAGTGCTAACCACTGTGAAACTCTGCCACCCATTTGTCTCCTGTTCACACCCCAATCTAAAAC TAAACTGAAACCTCAACATCTGACTGCTTCATCCCCTGGCTCCTTCCATTAACTACATCATCTCATTAAG CTGAACACGCTGTCTCTAATTATCTATTCCAGGAAACCCTCTCAATATAAACAAAAGTCAATTACCCCAA ACTTGTAGGATGCTTTAATTGCACCTCTTGTAGCCGCAAAACCTGGCTGTCTTGCAAACTAGAATTTTAA AAAACGCTACTGCAACAGTCACACACACTAACCCAGGATTTTAACCCTTACGGCACGCGGTACATACAGT ACAATATATCTGAAATCCCTACATTCATCACAGATACCACACTCGGTCAAATATTTTCTTGATGTCAAGA GCAGTCACTCTCACCTCACCTCTGGAATTCAACTCTTGGGTCCATATTTGTGGAGCAGAATGGTTTTCTT ATTCATAACTGGAAACAATAGTTTGAAGATTGCTTTTTAGACATGAGTTACATGGATGAGAAACTGGGTC AATCACTCACATGTACTCACAAGAgAgTATAAGCATGTCAGAAATCAGgGATGTAAGAAATTGAAAAATC ATCTCTAGCAAGGACTGAATTAAATGCAGGTGAGAAGAAACTATTGTAAAATATCTATGTGCAAAGGTCT GACACACATAATAAATGTTTGATTACGTGGGTTAGAGGTATACAGAGATACCAAGAACTGCACTGGGTGT TTCTGGGATACACTATGTGCCCAATCAGGGTAAGGCTAATTGCATCTTACCCAATGCCATATTGCTTTCG TGGGTCACTAAGAAAGTGATGTCGATTGCACTCACTGCAGGGGTAATCTGTCACCAGGCTGTGTCTAACT CAAAGTCTAAAGACCGATGCTGGGGAAGTGTAATTATTTAATTCAGTGTTTTTCCTCACTTTAGATAATT GATGAGGATAAGGAGGAATGCTTTGTTAACAGGTGGAATTTTCTAGAACTACTCCTCCTCCTGACGTGCG ACCAGGTTTTCAGAACGGGTTTAAAAGGGCAGGAGGTTAGGAGCTGTCCTCAGAGACAGTGCTGAAACGC СCTCTCCACTTTCCGTCGGCTTCTTCGCCTTGTGCAGA ATGTGGTGCAGCCGCCTTCAGTTGAGCCTGG

$\begin{array}{lllllllllll}M & W & C & S & R & L & Q & L & S & L\end{array}$

CTCTCCTCTCCATCGCCCTGGCAGTACTGAGTGTCAGCTCAGCCCCCACAGACAACAGGCATCGCGAAAT
$\begin{array}{llllllllllllllllllllllll}A & \mathrm{~L} & \mathrm{~L} & \mathrm{~S} & \mathrm{I} & \mathrm{A} & \mathrm{L} & \mathrm{A} & \mathrm{V} & \mathrm{L} & \mathrm{S} & \mathrm{V} & \mathrm{S} & \mathrm{S} & \mathrm{A} & \mathrm{P} & \mathrm{T} & \mathrm{D} & \mathrm{N} & \mathrm{R} & \mathrm{H} & \mathrm{R} & \mathrm{E} & \mathrm{I}\end{array}$ CCTGCAGAGAGCCTTGGATCAGGTAAGACCGATTCCAACTCACCAGATCTCACCAGCTGACATCAGGGGA $\begin{array}{llllllll}L & Q & R & A & L & D & Q\end{array}$

AGTATTTCCAAGgGAAAAATAGCAGTAGAATCATAGAATGGTTACAGCACACAAGGAGGACATTCAGCCC GCCACTTCCATGCTGGTTTACTATCACATTACATCTAGTGTCTCTTCTGCGACTTTTACACGTCGCTCTT TTTTTTCTCTCTGTTCAGATACGTCTTTGTTCTTTGTATCCAATTTTCCTTTGAAACCCATATTTGAAGC TGCCTCCACTATTCGACACATTGTTTTCCAGCTCCTAACAATTCGCTGGAAACAAAAGGTTTTCCTGATG TCACCTTTTGTTCTTCTGGCAGTTAACTGTTTTCCTGTGGATCTTGATCCTTCCATCATTTGGGAATAGT TTGTCCCAATCTACTCTGACCAGACCCCTCATGATTATGAACAACCCAATCAAATCTTCCAACATTAATT 
TCCCCAAGTCCCAACGTCTCAAATATATCCATACAACTGAATTTCAACAGAATTGTGACAACGCAGAAAG AGCCCTTTTGACCCATGGTGTTTGCACTGGCTCTCAAATCAGCATCATGATAATGAAATGAAATGAAAAT CGCTTATTGTCACGAGTAGGCTTCAATTAAGTTACTGTGAAAAACCCCTAGTTGCCACATTCTGGCGCCT ATCCAGGGAGGCTGGTATGGGAATCGAACCATGCTGCTAGCCTGCTTGGTCTGCTTTAAAAGCCAGCGAT TTAGCCCAGTGAGCTAAACCAGCCCCTATTTTGTGCCAATCCCTTGCATTTTCCCCTACTCCTGAACATT GTTTCTGTTCAAGTAACCATCTAAAGCCCTCTTGAACCTCTCAATTGAACCTGGCTCCCCCACACTACTA GACAGTGCATTCCAGACCCAACCACTCACTGTGTGAAAAAAAAATCTAACATTACATTTGTTTCTTTTAC AAATCGCATTAAATCTGTGCCCTCTCATTCTTGATCTTTTACCTGCGGAAACAGTTTCTCCCCGTCTACT CTGTCCGGCCCCCTCATGGTTTTAAGCATCTCTATCAAAGCTCCTCTCAGCCTTCTCTCCAAGGGGAACA GTCCCAACCTCCCCAATCTATCCTCACAACTGAAGTTTCTCATCCCTGCAACTGTTTTTGTGAACCTCTT CTGCCCTCTCTCTACAATACATTCACATCCTTCCTATGGTGTGGTGTACAGAACGGTGCACAATACATCA ATTGAGAGTCTATGTTAATGTCTTGTATAAATTAACATAACCACATTACTCTTGTACTCTATTCCCTTG TTTCTAAAGCCTGGTATACTGTGTATTCATTAACTGGTCTCTCCATCTGTTGTACCACCTTCAATGATCT ATACATACACACAGGTCTCTCTGCTCCTGCACCCCTTAAGAATTTATGCTTGATTTTATATTGTCTCTC CATTGTGGGCAGCACGGTAGCACAGTGGTTAGCACTGTTGCTTCACAGCACCAGGGTCCCAGGTTTGATT CCCGGCTTGGGTCACTATCTGTGTGGAGTCTGCATGTTGTCCCCATGTCTGCATGGGTTTCCTCCGGGTG CTCCAGTTTCCTCCCACAAGACCCAAAAGACATGCTGTTGGGTGTATTGAGCATTCTGAATTCTCCCTTC GTGTACACAAACAGACGCAGGAGTGTGGCAACTAGGGGCTTTTCACAGTAACTTCATTGCAGAGTTAATG TACAACTTGTGACAATAAAATATTATTATTATTATTATGTGACTCCTCCCAAAATCCATCACCTCACACT TCTATGCACTGAACTCTATCTGCCATCTATCTGCCCACTCCACCAACTTGCCTATGTCCTTTTGAGGTTC TATACTGTCCTCCTCACAGTTTACAATACTTTCAAGTTTTGTATCATCCACAAACTTTGAAATTGTTGGG CAGCACAATGGCGCAGTGGTTAGCTCTGGGACTGCAGCACCGAGGACCCGGGTTTGAATCTCAGCCCTGG TCACTGTCTGTGGAGTTTACACAGAACATAGGGCAGCACGGTAGCATTGTGGATAGCACAATTGCTACAC AGCTCCAGGGTCCCAGGTTTGATTCTGGCTTGGGTCACTGTCTGTGTGGAGTCTGCACATCCTCCCCGTG TGTGCGTGGGTTTCCTCCGGGTGCTCCGGTTTCCTCCCACAGTCCAAAGATGTGCAGGTTAGGTGGATTG GCCATGATAAATTGCCCTTAGTGTCCAAAATTGCCCTTAGTGTTGGTGGGATTACTGGG

\section{(scaffold 604248)}

GTACGAGACAAGTTTTTACACAGAGGGTAGTGGGTGCCTGGAACTCGCTGCCGGAGGAGGTGGTGGAAGC AGGGACGATAGTGACGTTTATGGGGCATCTTGACAAACACATGAATATAGTGACACAGTGGTTAACACTG TTGCTTCACAGTTCCAGGGTCTCGGGTTTGATTCCTGGCTTGGGTCACTGTCTGTGTGGAGTCTGCACGT TCTCCCCATGTCTGCGTGGGTTTCCTCCGGGTGCTCCGGTTTCCTCCCACAGTCCAAAGGCGTGCAGGTT AGGTGGATTGGCCGTGATAAATTGGCCTCAGTGACCAAAAGGATAGCTGGGATAACGGGGATAgGGTGGA GGTGTGGGCTTAGGTAGGATGCTCTTTCCAGGGGATGGTGCAGACTCGATGGGCCGAATGGCACTGTAAA TTCTATGATCCTATGGTGAGGATGGGAATAGAGGGATACGGACCCCGGAAGTGCAGAAGATTTTAGTTTA GACGGGCAGCATGGTCGGCGCAGGCTTGGAGGGCCGAAGGGCCTGTTCCTGTGCTGTACTTTTCTTTCTT CTTAAGCACCTAACGTCAGCACCAAGCAACTGAAACACAAAGGATTCAAATTAATTTTGAACAGGAGTCC TGTTGGATTCGAGATTTCTCTTTCCACAGAAGCTGCCGGACTTTCTGGATTTCTCCAGCATTTTATTATT TTACATTGCACAATCCAAACTTCTGTTTCTAACAGACTTTAATTGCTTCCTTTCAGGAACTTCTGAAGGA $\begin{array}{lllll}E & L & L & K & D\end{array}$

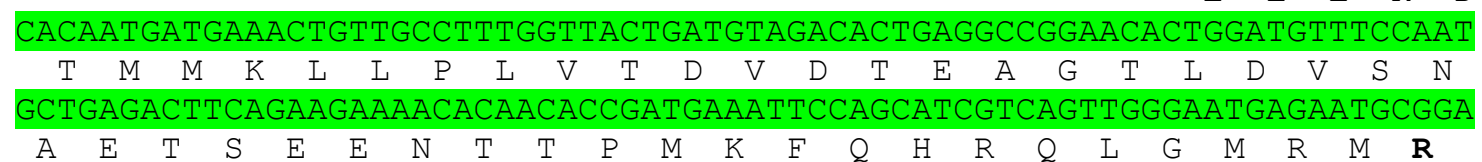
AAAACTGCAAGAACTTCTTTTGGAAAACCTACACTTTATGCTAATCCACTGTACCCAGTGAGCCCTTCTC $\begin{array}{lllllllllllllll}\mathbf{K} & \mathbf{N} & \mathrm{C} & \mathrm{K} & \mathrm{N} & \mathrm{F} & \mathrm{F} & \mathrm{W} & \mathrm{K} & \mathrm{T} & \mathrm{Y} & \mathrm{T} & \mathrm{L} & \mathrm{C} & -\end{array}$

ACTGGACCCTGCTGTACCCCACTGCACCCCACTGACTCCATCCCACTGTACCC 


\section{Catshark SST5 $=$ SSb \\ (scaffold 888145)}

GTGGAGAGTGTACGGGCGTAGGGGAAGAACCTTGAGGTGGGCGATGAAAATCTCTCTTTCCTCACATCGT ATtGAGTGGGGGGTGGCAGGGGGCATGAAACAGAAATCAGATAGAAAGCAAAATGGGTTTCTCTTCATTG AGTGAGAGGGCATGCCNACTGATTGGTACACCGTCCGCCCAATTAAATCGCAGCACCAATCAGAGAGTGA GGAGCGTGGGGGGCTTTGCTTGTGACGTGATTGCTACCTCGCCTGAGGGGGTATATAAAGCAGGTAAATC GCAGGGCTGGAGTTGGTCAGAGCAGTTACACAGAGAAGCTCGCCTCTCGCCCACACTGCCCGACGAGACA GTTTGCCAATCGCTCCCATCCGCAGTAAGAAAATCAGAGCTCGAGATGCCGAGATCGCAGACTCAAGTCC $\begin{array}{lllllllll}M & P & R & S & Q & T & Q & V\end{array}$ TCCTCACTCTGGTCACTGTCGCTCTGCTGTTACTGCGGGGATCGAGTACAGCCAGAGTGCAGGGGCTGCC $\begin{array}{lllllllllllllllllllllllll}\mathrm{L} & \mathrm{L} & \mathrm{T} & \mathrm{L} & \mathrm{V} & \mathrm{T} & \mathrm{V} & \mathrm{A} & \mathrm{L} & \mathrm{L} & \mathrm{L} & \mathrm{L} & \mathrm{R} & \mathrm{G} & \mathrm{S} & \mathrm{S} & \mathrm{T} & \mathrm{A} & \mathrm{R} & \mathrm{V} & \mathrm{Q} & \mathrm{G} & \mathrm{L} & \mathrm{P}\end{array}$ CGATATCTTACAGGAGAGGAACGACGAGGGGACTGAGGTAAGACAGATACCCCTATAAAATCTCCATCCT $\begin{array}{llllllllllllllll}D & I & L & Q & E & R & N & D & E & G & T & E\end{array}$

GTTGCGCTGGCGCAGGTTAACCCCAGACACTCCATTCTGCACTCTATTTAACCCGTTAAGATCCAGCACT TAACATAATTAAATGCATAATTTTGTTTAGTTTATTGCTGGAATCCATCT

\section{(scaffolds 316000)}

TTCGGCCCTCGATGTTGCGCCGACCTGTGAAACCATTCTAAAGCCCATCTACACTATTCAAATGCCAGTG TTGTGTTGTGTTTACAGGAGCTGTCCAGGACCATGGTGCTGAACCTCCTCTCCGAGCTGCTCCATGCTGG $\begin{array}{llllllllllllllllll}E & L & S & R & T & M & V & L & N & L & L & S & E & L & L & H & A & G\end{array}$ GGACAAGGGGTTGCCCAGCACCGAGAGACGGGAGCTTCCCCAGGCTGAGATCGTCCGCCGGTGGAACTCA

$\begin{array}{lllllllllllllllllllllll}\mathrm{D} & \mathrm{K} & \mathrm{G} & \mathrm{L} & \mathrm{P} & \mathrm{S} & \mathrm{T} & \mathrm{E} & \mathrm{R} & \mathrm{R} & \mathrm{E} & \mathrm{L} & \mathrm{P} & \mathrm{Q} & \mathrm{A} & \mathrm{E} & \mathrm{I} & \mathrm{V} & \mathrm{R} & \mathrm{R} & \mathrm{W} & \mathrm{N} & \mathrm{S}\end{array}$
CCGCGGGACCGCAAAGCAGGCTGCAAGAACTTCTTTTGGAAAACCTTCACCTCTTGTTAACGTGCGGAGA $\begin{array}{lllllllllllllllllllll}\mathrm{P} & \mathrm{R} & \mathrm{D} & \mathrm{R} & \mathrm{K} & \mathrm{A} & \mathrm{G} & \mathrm{C} & \mathrm{K} & \mathrm{N} & \mathrm{F} & \mathrm{F} & \mathrm{W} & \mathrm{K} & \mathrm{T} & \mathrm{F} & \mathrm{T} & \mathrm{S} & \mathrm{C} & -\end{array}$

GAGAGAGGGAAAGAGAAAGTGAGCCGGCGGGGGGAGAGATATAAAGAGAATTCCTCCCTCTCCCAAAACC CATCTTCAGCTCCTGAAGGGAGGCAAAAAAAATCAATTGCCTCTAACTTGTAGATTTTAAGATTCACAAA TCCCAACCACCTCCGTCCCTCGAAAGATTGCCTCCCCCTTTGGGCTGCCTCTCCGCTGAGGATTTCTCTG TGTCTTTATCCAAGGTGTTTGTAAATGATTGACTTGTTTGCTTTGTTCTCTGAGCATGGACTTGCTCTCA CACAATAAAATATCTTTATTTCTCAGCAACCTCCACGACTTTGTGTTCCTTTCTGCCCTCTCCCTGACTG GGAATCCATCCCTGCACCACCTTCCACACTCATACCCAGCTCCCCTCTAAAAATCTAATCGGTTATGTGA ACATTTTTGGGTTGTGGGGGGTGAAACCCACGCAGACACAGGGAGAATGTGCAAACTCCACACGGACAGT GACCCAGAGCCGGGATCGAACCTGGGACCTCGGCGCCATGAGGCAGCAGGGCTAACTCAATGCGCCACCG TGCTGCCTTCATAGGCTGTTAATGACCTCATCCTCCCTAAACAAGCGAAAGAGGGGTTTGCAGCTTGTAA CGACTATGAAGTACCGTCCATAACTGTATTCCCAGAACTTCTCCACTCGGAAAATTACGGCTAGTCCCTT TTTCTCCATATGAGAATTAAGCTTTTTCGGCATCAGTCCGAGTCCTCGCACAGGCAATAGGCCACCTTAA ATGATAAAACCGCTCCGATTCTGGGTGAGAATGATCGCTATTAGCGGGGAGTGGGGGGAGGGGGGGATTC TAAATGAATAAGAAACTGGATGACTGGAAAGCTAGTTTGACCTGTTAAAAGGTTTACTTCTGATGTTCTC TCCAATACCACCTCTGCTGCTTCTTGAGCAATGTGTGTAGGGGTGCCAACATTACAGAAAGATTAGGAAT AAATCTTCCGTAACCATTCACCATTCCTGAAGAAAATTTCAGTTCCACACATTTTTGGGAGCTGGTGCTT CCTTCGATATTCTCCTACATTGGTTGCAGCCCTTGGGCGTCTACCCTGAACCCCAAATACTTCACTCGAT GAACCTGGAACGTGCACTTTTCAATTTTTAAGACTTTTTAAAACTTCTCTAATTCTAATTGTTGGCACGC TAGCCTCATACAAAGTTTAGTGTCAGTTAAACCTGCTAATTTAACATATAAGTCTTCGATTCTTGGTATT GAGTAACTGCCCAATTTTGCTGCCAGTTTCACGGTTAATTTATAACCGCACATATCTCAATGGTTCCATC TGGTTTAACCACAGGCACGATGGGTGCTGCCCACTCCGAAAACTGCATAGATCTGATGATGCTTCATTCT TCCAATGTCTTGAATTCAGGTTCAACCTTCTCATGTAAGGCACGGGGGTATGGGCCAGGCTCTGTAAAAC TAGGATCCACAAGCAAATTTAATTTGACGATAAGAGCCCCAGGGGAGACGATAGCGGCATTCACGGCGTA CCTAAGACAAACAGCTGAGTGTTGCAAGCTCGGGTCTGCGCTCAACGACATGTTGAGAGACCGGTTCATC CATGGCGTTACCGACATGGCCAAATTACTAGCAGAGGCATAGATTACCTCATAATAATCTTTATATATTT TATAATCGTTATTAGTGTCACAAGTAGGCTTACATTAACACTGCAATGAAGTTACTGTGGAAAACCCC 


\section{E. Catshark SST6 $=$ SSC (scaffold 66610)}

...GTAATAAGGGAAGGAAAAATACACTAAGGTCCCGGGTCCTGATTGCTACACACCAATGCCAAATAAGGC ACCAAGATCGGGCTGTGATGCAGCCACAGTTGAATAGTTTGCTAACATCCATGGTCCATGCTTGCATAAG AAGAATGGGCACTCGATAGAGATACTGGAGTGCAGTCAGCATCTGTGAAGCTGTACTCAGTAAAGATAAA ACATTTTCAGAGAAGCAGGTGAAGGAACATGGAATGTGTTTCTGGTCTGAAAACATAGAATCCAATTCAC TTGACATTGCTTTTTCTTTAAGTATAAAACACAATATCAGATTTGGGACTTTTCAAATCATCAAATAATA TTCCACAAATGGCATCAAGAAAAAACGTTTAATCATATTTTAATTTCCATATATACCATGATAGATATTT ATATTTGCTCAGTGCTGAACCTCTCCAACAAATTGTTAGCACAAAAAGAATTCTGACTGGGAAATGTCCG ATGTTAAATGTTGCCTGAGCATTTAAAATTTGTCCCTGAAGCAAAGGTTCTTGCCTTAAGTCTGGAGACA GAAATTCAACATTTAATTTAATTCAGAATTTAAAACTTTCTCCTCAGTGCTTCTTATCTTCAGGCAGAAA ATTGCAGAAATTCAACGCAATAAGACTCTTGTATTGACCGATGTGTTTGTGAAGCAAACCTGGTCAGCTA AGGGTTAATCTTGGACCGAGTGTAATTGTTTGGCTTTGATGGAGCCCTGATGTTTAATGAAGCTTTGGTC TGTGCGTCAGTGACTGGGGCTGCGCTGGGTTATATAAAGGGTTAGAGATTGTCAAAGCCACACAGAGACT CCAAGGTCGAGGCACATTGAGAAGATCTCGGCCAGGCTCCGCTTCATTCCAACTCCACGAGATCGAGAGT GTCAGCATGCAGTTGCTGGGAACGGCGAGCCTCCTGTCCATGCTGCTCATCGTGTCCAGTGTGAGGGCCA $\begin{array}{lllllllllllllllllllll}M & Q & L & L & G & T & A & S & L & L & S & M & L & L & I & V & S & S & V & R & A\end{array}$ CGGCACCTTTGGAAGATCGGCTGAGTCTGCGGGCAAATCAGGTGAGTGAAAACTTAAGCGAGGGGAAAAT $\begin{array}{lllllllllllllll}T & A & P & L & E & D & R & L & S & L & R & A & N & Q\end{array}$

CTGCAAAGGGTACACGGCCGGAAGGGAATGACAGAACGAAGAAAAGAGAGGGAGACGACAAAAAAGAACA CACAAGACAAAGCAAGGAGACAAGGAGCGAGAGGCAGATGGCGTGGGAGAGAAGTCGAGGTCGATATGAA TCGGGGTGACAATTACAGAAGGGGGAAAGGCAGCTAGAAAATGAGAGGGGGATGGAGGTAGGAAGAAAAC CTGAGCAACGAATGATTTCAGAGAAGAGGAAATCTGTGAGAAGAAAAGAAAGTTTCAGTCAGGTAAGTGG CATGGGTGTGGGTTAGAGTGAGAGGAACTGAAAGTAACAGTGACAAAGGCTGGGGATAAAATGAGAGAGA GAGAGAGAAAATAAAGGTGGCGGGGATGGCAGAAAGAGACCTAGCAAAGGGGCAAGAAGTGTGGGTGAG GGGGAAAAAAAGTAGAGGGATGAACAGAAAAAAATGTGAGGGAGAAGTAGAAAATGAGAGTGACTCTGGA AGAGCGACAGAGGTGGAAATAATAGAGAAAAAGCAACTGACAGAGGTTAAAGGGAAAAAAATAAATGAGA CAAACATGCACACCAGAAAGTGAGAATAACAGAGAGGTGGTGAGAGAGGGACAGAAGAAGAGTACGAGAA TTTTTTTTTGAGTTGGCTGATATCCATGACTGGTTTCTTGGGGGGGAGGGATTGTGATAGTTATCCCGG GATGCACAAAGATTTTAGTGTCTGAGTCTGCCTCTGGAATAAGAGTTAGTCATGCCGACACTGTACTCAC ACAAGCAGCTTCCCACCCCTTTAATCACATTTAGTTTTGGTGCTTTAAGTTTCCTTGGAGATTCTGTTCA CTTTGATGCAGTACCCCTTTAAATGGCAGCCTCTTCATTTCTCCCCCAACTTGCTGATTTTAGATTTTGT GGTTACCTCAAAAGAAGCACAACCAGTTTCCCAGAATCCGACTTAAACATGCATCAGTGTAAAAGTGGCA AAAGAGATGCAGCAAGAGGGAAAAAGGACAGATAATTTTTTTTAAAACACACAAAGAGAGAAACTAAATG AAAAGATTAGGGTGCCAGAAAAGAATCTTTTCATTCCGATGCTGAATGCGGAGGAGACAATATCCTTTGA ATCGAAGTGTAGCATGCAGTAACTAGGAGAGTGAGTGACATGAAGCACAAAAAACAGAGCAAGACAGGTG GAACAGTAATAAGTAAGCCATGACAAGCAATAAAATGCAAACTGTGTGTTCTTACCTCCCTGGCGCACAA TGTTCGAAGATGCCTACCCCTTATTATTAGTCTAATAGGTCACCTCGGTCAATGTTTGTCTCATGGTAGC TTAGCTCCACTCACTGACGCCAAAACGTTCCCTCATCCTTCACTTCTCAGTGCTGTGTGGCTTAAAATGA TCCCCAAACTATAAATTACTGTCGGTGTTGTAATTGTATGAAGACTTTGTGTACTCCCCTCTGATTCGCC TCTGGGTTAGCAGAGCTGTATACGTGCGAGTGTGGACTTTTGAATAGATACTCGACATTTTACCGTGTGT TTTCTGTGTTAACTTGTCCTTTGCTGCTATCTCCAACAGGTGCTCCTGTCCCACTTAACCCCCATCTCAT TATTCAGGGGGTTTCTGTTCAATTATACATGACCGAGGGCAGCAAGTGGGAGCCCCCTCCCTGTTGAGTT GAAGGGGAGGGGGGTCCTTGGCAGGTGAAACAACTTTGTGCTGGATACTTGGGTAAGGATAGACTTAGAG ACAATGGGCTGGATTCTCCGTCCCGGTTCAGCACGCCGGCACGATGCTCCGTTTCGCCAGCTGGTCACTG TGGGCAGGTCCCATACCGTCGGGAAACCTCCGGGTTGCCAGCAAAATGGAGAATCCCAACGGCGGAGAAT CTAGCCCAAAGTTAAAGAGAGAGCGAGTTTTATATGATCACGAAGGAGTCCACATCGAGTAGTTCAAAGA AACTTAGTTTATTTACAATCACTATTATATACATCACACGGCAGATCCCAACTGGGTCTGCCTTGCTGAT GCCAAACTGGCCAGCTTTATACATCGTCCAATAATATATTGTTTAGAGGTCCCCCACACCCTTAACGGGG GAGCTCGTATTCCGCAAGGTTCATGGGGAAGCTAATTGCCCTCACCCCTTTAGACCCTTGCGGGTTATAA TGAGAGAGAGGGATTGCAGCCGTGAAGATCGGTCTCTAGATTAAATATAAAATGCCTATCAGGTACTGAT TTCCAGCCAGGCGGTGCTATAGCATCATTGCTCAGTCCATGTGGACACCCCTTCCATTTGAAGACGGCGC TAATTTAATGTATCTCAGACAGATGAAGGGCAAAGCCACTTTTCCCTTGACAAGTATACGGAATCGGGCT TGATTTTTGACCCCGTTTCTGTTTATCTAAATGTTGATCAGAAATGACCGGTTTTCTCATTTTAGACAT TTAGACACAGGTACAGAGCAAGGCGGCTCTTTACCCCCCTACCTTGTCCCAGCAATTATTTTCCCCCCTT TCTCAAGACAGAACTGACCCACCGCTTTCACAAGGATGAATTTTTTCCCATTCTGTAGTCTCTAAGTTGT GATTTGCTGTGGGTGTTTTCGTGCTTGAACTGTGGCCGATTCTGTCCACGAACCTCCGGAAACAAAGCAT TGGCTTGGAGTTCCCGTTTAACAGAGAGTCGCTAAACATTGTGAAGCTTGCAACCCATATCCCCCCCCCC TTAACAAAACAGGATGGCCATTATCACAATGTATTATATTTTGAGATGGAGTTGATAGTCACTATATGTT GGGATGCATTAGCAGAAGATTCTAGCACATCATTGTTTTACGCAGGTTCAGTATGTATCGCAGCTCAGTA ACTTACGGCAAGGTCAAAAGGTCACGAGAGCAACTTGATCCAATCTCTAAAATTCTGGCAGCAATGAATC 
ATAGAAACCGTACAGTGCAAAGGGCGGCCATTTGGCCAATCAAGTCTGCACCGACACTCTGAAAGAGCAC CCTCTAAGGCCCAAACTCCATCCTATTCCCGTGACCCCACCTAACCTTTCGACGTCGGGGGGGGGCAATT TAAAATGGCCCGTCCACCTAACCACATCTTTGAACACTAAGGGACAATTTTTGTACGGCCAATCCACCTA ACCTGTACATCTTTGGACTGTGGGAGGAAACCGGAGCACCCGGAGGAAACCCACACAGACACGGGACAAA CATGCAAACTCCACGCAGACAATCACCCAAGACTGGAATTGAACCCAGGTCCTTGGCATTGCAGGGCAGC TAAAAACTGTGTCACCATGCTGCCCATAATGCTGCATCTCATCCAACCCCTCAGTGGAGGGAGGGTGTAG AACACCAAGTGAAGGATGGGGTGGAAGGATGGGGAAATCAAGGGCTTGGGCGGCAGGATTTGTCTGGAAA TACTCTTGTGCAATTGCTGTAATTGTAACAATTATGAAAGCTAATTAAGTAGCTGACGATCAGATCCTTT TGTTAATGCTACGCGCTGAAAAACAGTTAAATGAATAAATGGGTGAATAGAAACATGTTTAGAAAGGGCT GAGGGAAGCAANNNNNNNNNNNNNNNNNNNNNNNNNNNNNNNNNNNNNNNNNNNNNNNNNNNNNNNNNNN NNNNNNNNNNNNNNNNNNNNNNNNNNNNNNNNNNNNNNNNNNNNNNNNNNNNNNNNNNNNNNNNNNNNNN NNNNNNNNNNNNNNNNNNNNNNNNNNNNNNNNNNNNCTGGGATTGTGGGTGGGTGGGGGCGGTAGCTACC AGCTACTTGATCCTCTTTCCTCCTGCTGCGCACCAACATAGGTTTTCTAGTGGAATGTAGAATTTTCCTG GAGACTCGGAGGTTAGCATATATGAACATATCAATTAAGAGCAGGAGTAGGCCACTCAGCCCCTCTAGCC TGCCCATCATTCAATAAGATCATGGCTGATCTGGTTGCATCCTCAACCCCACATTCCTGTCCAGAACCAA TAACCTTTCACCCTCTTGTTGATCCAGAATCTATCCAGCTCAGCCTTAAAAAACATTCGAAGACTCTGCT TCCACTGCCTTTTGAAGAAGAACGTTAAAGAGATCCTCTGAGGGAAAAATATTCTCCTCATCTCCATCTT AAATTCAGTAGCAGCGGGACTGCTTTGCTTCCCATGGAACCCTACAGTGCAGAACGAGGCCATTCAGCCC ATCGAGTCTGCACCGACCGCAGAAAGCTAACCCCATCCCAGCCCACTCCTCCACCCTGTCCCCGTAACCT AATGGCCCCAGCTAACCTTTTTGACACTAATGGGCCATTTATCATGGTCAATTCACCTAACCTGCACATC TTTGGGCTGTGGCAGCTCCTGGTGGAAACCCACGCAGACATGGGGAGAACGAACAAACTCCACACAGTCA CCCAACGTCGGTAAATTAAAATGTTAATTAAAAACTTTAATTAATTGGGTTTTTAATTAACCTGAAGAAG ATTAACTAATTCCGATGATCCATTGCTGAGTTTTATGATCTCCCTCTCATTTTGGAGTGAACATAATGCT CAATATATTGGTACTGTTGCTTTAAGAATAGCGAATGCCTCACGGAGCTGCTATGGAGCTGATCCACATG CTCCAATCAACGCTGTGACCGAAGAGAACATTATTCTGTGAGCACAGGAAAACAGAGTAAATCTCTTCAC CACTCTGTACTGTGTATGACTATTTTTTAAAAAATGCACAGGGCAGAGAGAGAGTGCTAATTGTTGAGCT CAGCACTAAATGACTTCGCATGGGTGGTAGTGGCTGCTCATCTGTAATATCCTTGGTGTAACCCAGCTTC ACACGTCGAGCTCCATTGTCCTTTCCACCCGTTCCGCTATGAGAAATCACCCCGGGTTCCCGCTCATGGT GAAATGTTTAACTTGCATCTCCCCGGGTTATATAAATATGTACAATAACAAAATGCAATCATTAAATCAA CAACAGAAATGAGGCAGAATTTTAAATCCTGCTTTGTGAAAACTTTAAAAGTAGGCTGTCATTTAAAAAA ACAGTTTATCCAGAGTTATCATAGAATGTGACAGCAGAGAGGTTCAGTCCATTGTGCCACCACCACGTTC CCCCTCCACCCCACCCCAGATTCCACCCTAAGGGCGGGTGACATCCTCACCATTATCCTGTGACACCCGC AGAGAAGCAGGTGGGAGCAGGCGTCAGGCAAGCACAGAATCAGGCCTCCATGGCTTGCTGTGCCTCACTC ACCAGGCTTGAGCTGCTGGAGCATAGTGGGGgGA GCCGACAAAAAAGGACTATCCGCCAGCAGGAGCTAT CACTGCATTTGGTGGAGGAGGGGGGAAAATGCATGGGTGGGGTGGCAGGAGAATCAGAAAACAAGGTGCG ATTTTTCACTGTTAATTAGCGCTCGCATCTCGGAGTCACAAGACCATAAGACGTAGGAAGGCAACTCACC GCCACCTTCTGAAGGGCAACTAGGGATGGGCAACGAATGCTGCCCTTACCAGCGACGCCCACATCCCGTA AATGAATTTTTAAAAAAGGAGCAGAAGGAGGCCATTCGGCCCTTCAAGTCTGCTCCGTCATTCAATGAGA GCATGGCTGATCTGATATAATCCTCAATTCCACTTTTCCGCCTTATCCCCATAACCCTCGATTCCCTAAC TGATCATAGCCAGAGGAAGTCTCACTCCAGATAATGCAGGCCAACATTTCACTGCAGTAGTGATGGCGTG CTACCCTGTTGGAGGTGCTGTCTTTCCAATAAGATGTTAAACCCGGGCCCTGTCTGTCCTGTCAGGTGAG CATAAAAAATCCCAAGACGACCTGATCAACACTAAAAAAGGCATTTTCTTCACCCATGGCACCTTAAATA GAATCGTCAAATTCTTGTGTGCTCAATGCTCTGGAGCACAATTTCTCACACAGGTAACTTAAATGTATCA TGTCCTGTGTTTGCCCGTCAGGAGCTGAACAAAGAAAGGAAAGAGGTCATTTTGAAGCTGTTATCCGGAC $\begin{array}{lllllllllllllllll}\mathrm{E} & \mathrm{L} & \mathrm{N} & \mathrm{K} & \mathrm{E} & \mathrm{R} & \mathrm{K} & \mathrm{E} & \mathrm{V} & \mathrm{I} & \mathrm{L} & \mathrm{K} & \mathrm{L} & \mathrm{L} & \mathrm{S} & \mathrm{G}\end{array}$

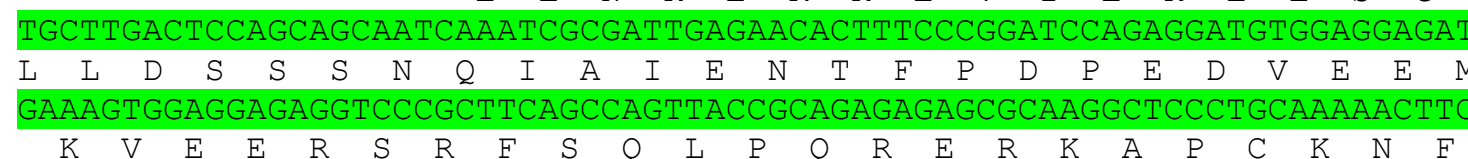
TTTTGGAAAACCTTCACCTCCTGCTGACGAGAGACAATGCCACCTGGCTGCCAACTAAAGCCGCCGAGAA $\begin{array}{llllllllll} & \text { W } & K & \text { T } & \text { F } & \text { T } & \text { S } & \text { C } & -\end{array}$

AGGAACTGATTTAGCACTGTACATAAGCTGCTTTTTATTTTATTCATCTGTGAGGGGTGGTGGAGGTTGG GGGGGGGTTAATATTTTCTGTCGTGCACAAGAAAAATAAAAAGAGCATGATTGTGTGATTTCACCGCAAC CTGTATGTATTTATTTTCTTTACCAGTAATGTTGTCGCGCTCAACCTGCCAGAGCAATAAAGTGGATCCG AGATAATCAAATCGATTCCATTATTTTACTGACTCAATTTTCCGAAGGGGGATTTGATGTTCTGTGGAAT TGAGGGTCTTGAGCATGGAAGGGGGGTGGAGGGGAGGGGTTCAGGAAAATAGTGAAAACCACGTAGAGAG ATGATTAGAGACAGCAAACGCAGGTCGGTGAGAGAGGTGAAAAGTACAAGAGAGAGTGGGCAGTACGAGA GAGGGGGAAATTGATAGCCGTCGAGTGCGTGGTCAGTTCCAGCCCCACTCGTCTCGGAGTCACAACGCAA GTGAATTAACCAATAATTCTGATAAAAATACACACCGTCTTTGGCCCTTGGCTGACCAATAATTACAGTC ACCAGGTTTGTAAATGTAAACACAATACTGTTTATTTATAACAAAAACTATCTTGAAACAAATGCAGTA... 


\section{Figure S5}

\section{A. Whale shark SST1/3 (NW_018029766.1)}

...AAGCAAGTCCAAGTGTTGCATCTGCCTCACAGACACCTATTTGATAGGCTACATCCAACACCAGTCTCA AACTACATCTCAGACTTCAAGGCACTGGCAGATAATGTGCAGTCACAGGTATCACATTGAGGCATAGTAA GTAGAGGACAAAATAAATATTTACGTACATTTGGTTTTAGAGTTATGTGGCACTAAAAATAAACTGTTTA TTTCGTACTTTCTTAGAGTAGATCCTGGCTTGCACTTAAATTCAAAAAGTGATCTTGTGTTTAAAAACAT TGGTGACCTTTGCTGTAGAGTTAGTGCAACAAATGTTCTCCAGGCTTGTTCCTGGGATAACAGAACTATC CCGTGATGATAGATTAGGCAAACTGGGACAGTATTTGTGAGTTTCAAAGAATGAGAGATGATCTCATTGA AАCCTACAAAATACTTGAAAGGATGGACAAGGTATATGCAGGTTAGACGCTTCCACAGTGTGACTGAGTC AAGTTACTGCAGTGTATCGTGTATTTGCACAAATGTCACTGCGCATCAGTGATAGAGTCAATATTCAAGA TGGTGGGAAGTGGTGCCAAGCAAGCAGGTGCCTTTGTCCTAGATGGTGTATGTTTCTTGAATACTGTTGG AGCTGCCCTCATCCAGACAATTGGGTTGTTTCCCATCGCACTCCATACTTGTGCTTTGTGAATGAAGGAT AGGCTTTGAGGAGCCAGGAGGTGATTTACTCACTTCAGGATTCCTAGTCTTTGACCTGTTCTTGTAGCCA CTGTATTTATACAACTGACCTAGTTCAAATTCTAATCAATGTTAATTCCCAGGATGTGGATGGTGGGGAA TTCAACAACGGAAATGTCACTGAATGTCAAGGATAACAGTTTCTGTCTTGCTGGAGATGGTCATTGCCTG ACGTTTGTGTTACATCCCAATTGTTAGTCCAAGACCTAAATATTGTACAGATCTCACTGCATTTTAGCAG TATTTAAAGAGTTACAAAAGTACTGAACATTGCAATCATTAGCGGACATTCCCACTTCTGACCTTATGAT GGATCATTGATGCAGTAACTGAAGATGGTTGGGCACAGGACGCTACCCTGAGGATCTCCTGTCATGATGT CTTGAAGCTGTGATGACTGACCTCCAGCAACCACAACCATCTTCGTTTGTGGCAGGCATAAAACCAACAA GTGAACATACATTGTCAGCGGATGTCAGGCTTGAGCTTGCAYTGGAGCAGCTTGTTTAGGGCCATGACTT GTTCTGAAGCACAAGTCTTCAGTATTATTGCYGGAATGGTGTCAGGCTCCAAAGCCTTTGCAGTATCCAT TGCCTTCAATCATTTCCTGATAACACGTGGAGTGAATCTAATTAGCTGAAGATTAACATTTGTGATTCTG GGCATTCTGGAGAAGGCCAAGATGGGTAATAAATCCTGGCCTAAGCCACGGATATCCTGTGCATGAATAA AAGCAGAATTCAGAATGTCCCTGGGATTCTATTAAAACAGCCATGTTTCTTAGTGACAAATTGTAAGACT TACCCATTCTTTGAACTAAGTGACAAGAAACAAGACAAAATTTGTTTAATTCTTTGCGGTAAACACAATA ACCATTTTGTAGGACCCTGTACTTGTGACTACATGCAGCATGAACCACTTTAGTGTCATTTGTTTATTTG TCTGTTTGTGTAGCAATAGCAAAATAGAAAGCTGCACTGTACACTGTAGTTTGATTCATATCCATTGCTT GTCATCAGGATAGCAACATAAGGCTACTCAGAATTTAGATTGAACTTGGAAGATATCACAAATTACTGTG AACACTTTGTTTTCCTGAGCCAGTGTTTATTACAACGAGAAATATTCTGATCATGTCCAAAGAGGGAATT TTGCTCAAGGATGGTATCTGTTGCTGAATTGAAATTGAAACTGGGGAGAGAAGAGGAACTATCATTCCTC TAATGGGCTAATGGTAAGATTACTGGGCTAATGGTAAGACTACTGGGCTAATGGTAAGATTACTGGGCTA ATGGTAAGACTACTGGGCTAATGGTAAGATTATTGGGCTAATGGTAAGACTACTGGGCTAATGGTAAGAT TACTGGCATAATGGTAAGACTACTGGGCTAATGGTAAGATTACTGGGCTAATGGTAAGATTACTGGGCTA ATGGTAAGACTACTGGGCTAATGGTAAGATTACTGGGCTAATGGTAAGACTACTGGGCTAATGGTAAGAT TCTTGGTAGTGTGGATGAGCAGCGAGATCACGGTGTCCATGTGCATAGATCACTGAAAGTTGCCACCCAG GTTGATAGGTTGTTAAGAAGGCATACGGTGTGTTAGGTTTTATTGGTAGAGGGATTGAGTTTCAGAGCCA TGAGGTCATGTTGCAGCTGTACAAAACTCTGGTGCGGCCGCACTTGGAGTATTGCGTACAGTTCTGGTCA CCGCATTATAGGAAGGATGTGGAAGCATTGGAAAGGGTGCAGAGGAGATTTACCAGGATGTTGCCTGGTA TGGAGGGAAGTTCTTATGAGGAAAGGCTGATGGACTTGAGGCTGTTTTCGTTAGAGAGAAGAAGGTTAAG AGGTGACTTAATAGAGACATACAACATGATCAGAGGATTAGATAGGGTGGACAGTGGGAGCCTTTTTCCT CAGATAGTGATGGCTAGCACGAGGGGACATAGCTTCAAATTGAAGGGTGATAGATATAGGACAGATGTCA GAGGTAGGTTCTTTACTCAGAGAGTAGTAAAGGCGTGCAATGCCCTGCCTGCAAAAGTAGTAGACTCGCC AACTTTAAGGGCATTTAAATGGTCATTGGATAAACATATGGATGATAATGGAATAGTGTAGGTTAGATAG GCTTCAGATTGGTTTCACAGGTCGGCGCAACATTGAGGGCCAAAGGGCCTGTACTGCGTTGTAATGTTCT ATGTTCTATGTTCTATTCCAGTTATAGTCAGGAGCCATTTCACGTGGAGTTTGGAGGTGATGAAATGGAT GTGTTGAGTTAGTGATGGTGCTCAGTTTATATTTTCTTGCCACAAATTCCTCTAATTGTAATCACTAATG TTTGGAAGGTAAGGTCCAATTCAACAATGAAGCTGGACCTTATCCTTGCCTAACGTCCACACAGATATCC TATGATAGTGCTGTGAAGTGGAAAAACCACTTGGGTTTTTGCTCTTTCTATTACATGAGCATTGATCCCT CCCACTCTGATCCTGACTGTGACCAGCATAAGCTGGTGCTGGATTTTGTTTTTAGTCCAGAGCAGATAAA GCCATCTTACCCTTCATCTCCATTCTCCTCACCACCCACATGCTCACACACCTCTCCCAATCTTGCTTTA TTGGGAGAAAAATTAGAATTAATTTGTTTCTTTTTAACTTTCAGAATTAAAACATGTAACTGAAGAGACT CTAAGCAATAGATAACTGATGTCAACAGTAGGCAATGCAAAGACATGTTGAATTTCCACATTTCAGTTTC TTGCAAACTTTACTTGCTTCCTTCTA

$$
\begin{array}{lllllllllllllll}
D & F & \text { L } & \text { K } & \text { D } & \text { T } & \text { I } & \text { I } & Q & \text { L } & \text { L } & H & \text { V } & \text { V }
\end{array}
$$

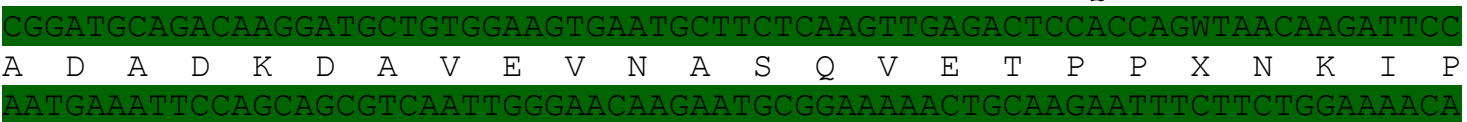

$\begin{array}{lllllllllllllllllllllll}\mathrm{M} & \mathrm{K} & \mathrm{F} & \mathrm{Q} & \mathrm{Q} & \mathrm{R} & \mathrm{Q} & \mathrm{L} & \mathrm{G} & \mathrm{T} & \mathrm{R} & \mathrm{M} & \mathbf{R} & \mathbf{K} & \mathbf{N} & \mathrm{C} & \mathrm{K} & \mathbf{N} & \mathbf{F} & \mathrm{F} & \mathbf{W} & \mathrm{K} & \mathrm{T}\end{array}$

TACACTTTATGTTAACACATTGTACTCCGCTCTACTGCACTGAGCCCTTTTCCACATTATCTCACTGTAC Y T I $\mathrm{C} \quad$ 
CTCACTGCATCCTGTTTGTCCACAGTGCCCTTCTCTGTGCTCAACGTAGTGGACTTTGCCTCACTGTACC ATAATGACTCCTTCCCATTGTGCCTCATCGTAGCCTATTATCCTCTACTGTACCTCATTATACCACATTG AАTTTCATTGTACTTCACTGTGTCCTACTGTACCCTAATGCACTGCTCTGTACCTCACAATGTCACATTA ACTCTTTCCCACTGTACCTACTATACAGTATTATACCACATTATGCCACATTGTAGTGCACTTGTACCCC ACTGTTCCACACTACAGGCAACTGTATCCCACTATAAAGTATGGAGTCCTTCACACTACATCCCAATCTA CCACAGTGTGTACTAATGAGCTCTTCCTATTTTATCTATTGTAATCTGACAATAGTTGCATTTAATCTGC AGTATAACTGAAGCTTCAGTTTAAACTATTTGACACACACATTCTTTACTTTTTGTTTTGCATTTGTACT CAAAGTCCTGCATTACGCTTTCAACATGCTGCAATAAAAATGGTTTCACATTTGAAGATTATGCTGCAGT СTCTGTCTAGTTGAAATTTATCATGGTGAGAATCCACAGATTGTAACCTTACCGAGAATCCAGTTATGGG AGTCCTGAGGTGGCAATTCCACGCAGTACTGAGGGAGCATTGCATTGCCATTGGTGTCATCTTTCTGACG GTCCGTCTGTTCAGCTAAACGTGGAAATGTCACAGCACCAGTTGAAGAAGACCAGAGAGTCTTTGCCATT AAATGACATCATGTGTGATGTGTTTCTGTTTGAATTCACATTCCCATCTGTACCCAGCAATCCTTTATT CCCTTGCATTATTTGTGGATATCTCCCCCGTAATTGTCCCATCTTCTTCTGTCATGCACCTATTTTCACA CAAAACCAAAAACTCGCATCCAAAATAGTGAATCCATAAGCAGATCTAACTAATTAATTCTGAAATGTGC ATCTAAAATAAACAGCTAATTGAAACCTTGATTTAATGCATATCATATCAAATTTGTGAAGACACTCCCC САTAтCTCATTCTAAAGCAAAATATTTTGGATGCTGGAAATCTTGGTTAAGAACAAAAATGCTGGAAAAA TGCAGTACACAAGGCAGGGCCCATTGAGAAAGAAACACATTTAACATTTAATGTGAAAATTAATTCCGTT TCTCTCCATAGGTAGTCCCAATTTGCTGAGACTTTTTAAATGTATGTTGTTACTGTGCAACTCATAATGA ATTTGAAATGAAACTCCAAATAGTACCTTTGTTATTAAATCTATAAATATGTTGTCTAAATACATATTTC TAATTGAAATCTGAAACTTCAGCTGTCTTGATGTTTTAAACAAAAGTTCCAATAACCATAAACATAACAT AGTAAGAACACTCAGCAGGTCAGGCCCTACCCTCAGGAAGAGAAACAGATTTAGGGAAAATAATGTAATC CAATTTTTAAGAAAGAAGCATTTCTGGAAACCAAAATATAATAATGACCAATATCAATTTGAAATGGAAG TGTTGCCTGATCAACTTAATTGGACTCTGAATCATCAGCAGAGTTTAGAAAAGACAATGAGGTAGATATA ATAATTTATATTTCTAAATGACATTCAAAAAAGTGACATTTAATAAACTATTCAATAAAGTATGCAGATA CATAGTACAGCATCAGAATGGATACAAATTGACTGTACACAGAAAGTAGAGAGTGGGGATAACTAGCTGC TCAAAGTAACAGAAGCTAGGGAAGTAGAGTTGTCTAGAAACAGGTAATGTTATTTTCAATTTACATTAAT GACTTTAAAATCAAAGCACAATTTAGAAATATGCAGATGTTTCCAAATTGCGTGGTAGGTAATAGTTGAT TTCAAGGCAAACAGAAACACATTTCAAGAGGATATTAATACAATTTTAGAATAGACATGTTGTAATTTTC AATACAGATTGAAAATAAAGAGCCATATATTTTGATTAAACATTAGACTAGAGGAAGCAGGATTGGCACA GACATGGATTAGTAAGAGTTGCACAGTAGCCCACTAAGGCCATAAGAAAGTAAAGTAAAATGCTCAGAGT TCCAAAGGTGAGTCATTAGACTCAAAATGTTCAATTTTTCTCTCTTTCCACAAATGCTGCCAGGCCTGTT GTGTTTCTCTGGCACTTTCCATTTTTATTTCAGATCTCTAGTATCTGCAGTATTTCGATTTTATTTAAGA TGAAGTAACGGGGAGATGGTATAGGAAAGTCCTCAGTGGTACCTTGCAGGTTTCTACCACCAGAGCTATT TTTCTAGCAGCAGAAAAGATATCAAATTAAACATCTTCCCAGAACCAAACTGAATAATTGGCTAGTTAAG GGGGTGAGTGGGGTTTGGAAAGGATGCTATGTGGGGCCGTGGACCAATTAAACCTGGCAGCCTGACAGCA GGCTCTGGGATTAGGCATCAACTACTCACAGCTCAACTGGTCATCATTACGGGCGCTACCATCAATGCAT CTTCATGCACTGAGTGCACAAGCACAAGTGGCCATCCTAGCAAGGTGCTGTTGAACTGCCAACCCTTTGA TTTGGACATTCATTCTTGGgGTCAGGCCATTGTACATAGGAGAAACAAAAGTTCCAACATAGCTGCTTAA CTGGTTGCAGAGGCCATAATGAAATCACATTGCCAACTGCACAGGGGTCGCACCAATAAAGGTAATACCT CACATCTATCAATGTTGAAATTTATTGGGCTTCATGTCAATTTCCTACCTCACTGTGTTGTACTTTCATG TCCTAAACCCTCACACCTCACTGAGCTGTAACTTAGGTTCTTATTCCCTCCCCACTCTGCTGTAACCCTG GATCTTATACTTAGTCTCAATTAGTTATAACACATGCGACCTATACATTACCCCATCACCCTCACTGTGC TGAAATTCTGGGTCTTATAATCTCCTTTATTGCATTGTAATCCTTGATCTATTACCCTCCCTCACTGGTC TGTAACTCTGGATATTATGTGCTTCCACTAGGAACTGTAACATATGCCTTATACTCTCCCTGATTGTGTT ATAACCTTGTGTCCTACAAACCACCAGCCCCATTCCTCCTTCAATGTGCTCTAACCCAATTACTATAACC СTTAATCTTGTACCGTTCATCATTATACTGTAATGCTGGGTCTATTAACTTGCACATCCCCACACCCCCA CCTCCCACTCAGTGCTATATCCCTGGGACTTATACCCTACCTCACTGTGCTGCACACCTGGATCTACTAC ACTCACTCAGTGCTGTAACTTCTGTACCTTCCCTTGCTGGTCTTATACTCCCCCCTCCCTCTCTGTGCTG TAACTCTGAATTTTGTACCCTTCCCCATTACGCTGCAATCCAGCCTCATTCAATAATTTGTGCACCCATG CCCCGAGGTGCTCTTGTTCCCCTCTGGAGTTGCATTCTCTATTTTATACTGTTTCTCCGTTCATTCCACC AGAATGAATCGCTTCACATTTTATTGAATTAAATTCGCTCTGTAACGTCTGTCAATTCCACCAGCCTTTC TACGTTTTTGGAACTTCTACACTATCCTCCTCACAGTTCGCAATGCTTTCAAATTGCGTCATCAGTACAT TTTGAAATCATCTAATGTACAGAAAAATCTCGATTGTTAATATAGATCAGGAAAATTAATGGTGATGAAA CTGATCAACTCGGCCCATCTGATGGCATCACCTGTTTGAAGGTGCCATTCTCTTGGTCCACTCTACAATA CAAGAGGTTAATGTCGTATTCCTGCCCGTGTTCGACACCGTCTCAATGTCAAGGTTGAGAAAAATCCCAG TTGTACGACCCCAAGTGAGATGCCTCATTGTCTGCCATTCTAAAATGATGTCCATTTAATGAAATAAGCG ATTCATTTCGACAGTTCCTTTCCATTTGAGACGGTTCCAGTTGACTAGAGTTTCCCCTCACTGATCAACA GTTCATTAATCTCCCGAGCCACTAGCACTGCACACTAACGGTGAGAAGACTTTCTGCCTTGCCTCGTGTC AAGATAAATGACATGTGGTATCATAGAGCTCCCTGGCGCTACTACCCGTAACAGGACAGAGGTCCACTCA TGCTGATAGGTTTCTACTTATCTTGTCAAAACACACCAACTTGTAAATAACAATTACTGCTCCCTTAATA TATTCATCAGCACTCAGCACGCAAGCCGCTTCCACAACCTATTTCTCAAGTTCCTTCCCATTGATTAACA 
GAAATATCTGCATAATAGGTCCTGGAAATTCCCTTTCACTCTCCCAATTCACTACAGGTACAATGGTTTA AACTAACATTATTCCGGTCGTTCGGTCTTTTGTGAACAAAGTATAGATTTTCCTGATGCGTTGCAAAAGA AACAAAAATTCATTGTCTAGAACATTTCATTTCATTTCTGCTGCCGGGTTGCACAGTGTCATCTGTAAAC GAATAATCTCAGTTAATTGCAGTCCCCTCCAGCCATTCTCACTCAGCAGAGAATCTTCCAAAAGTCAATA TTAAATAAAATCAGAATAGGCTACAAATAGTTCAGGCAGCGTCTGTGAGGGCAGAAAACTTGCCACTTGT TCATACAGCTACGGTTCAGTGGAAAACTTAACTACAGCTTACAGCAGAACCCAAACGTTGTGGGTTCTTT TGTTTTTACAAAACAAAAATCCAAAAGGTGAACAAAAGTGGACCGACCATCGAGACAGACAGAGGCAGAC AGACAACGACAGAAGCAGACAGAGACCGAGACAGACAGGGAGGCAGACAGAGACTGACAGAGATGGAGTC GCAGGCAGAGATAGAGAGACAGACAGAAAATGCAACTCTTTAGTCGTGATCAAGTCTGAATTATTGGTTC ACTTGACTTGAATTCTCCAATGAATCTTTATCCATTGCCTTGTTCATGCAAATAAGGAAGTGTTTCCCCG GGTAATGGTATATTTAATAGTCAGCCTGGTGCTGTTCTGTTTTCTTGTGTTCGCGCGGAGATACAGATAT TTAACATTGTTTGAAGCGATCAGGGATGGCGATATCATTAAAATTATGAAGGGTTGCAGGTTGTAAATCT CAACCAACCCACCAATTCAGTGTAAAAACAAAGTTAAATCTCAAGTCCACTGAGTTCGGTGTTAGCTTTA AGAAGAATACCAATCAGAAGGGATTGTAAACCCACACATAGAACACGGGGATAAAACCACAAAATACTAC ACAATGATTAGGAAACTAGCAAGTCTTTCCCCAGGAAACAATCCTTTACTGTGCCTCACGTAGTTTCTGC CCTCATAATCTCACACAATTCGCAGCAAATGATTTAGAAGTTGGAATCAGCTATTCTGCCTGTCTCCCCT GATATCCATTTATTAAAATTCAGGCTGAACTAGCTGTAGTCCAGTTCCATCTTGCTGTCTTGTCATAGGC TTTGACTGTCTTGACTATCAGAAATCTATCGAATTCTGCTTTGATTAATTTCAATGAGGCAGCCTGTGCT ACTTTCTGAGGAAAAAATGTTCAAGGACTAACGACCCTCTGACATCTTCCATCTTAGTCTTAAGATGGTG ACTTCGTTCTTAAACTGTTTCCGAGAGTTATTGTCTCCCTCATAAGTGGAAACATCCTTCGGCATCTCAC ACATTTTCATCTCTGGATCATTTAATTCTGTAATGAATTGGCCAGCACTCCCCTGGATTATGATCACAAA GTTTACTTGATAAGAATTTGTGCGTTGGAGAGATGTATTCCCTTTTCCGAACGAGCCCTATCGTCACTCC GACCTTTTGGCAGGATCGAATTATTAGTGGAGACCACCTCACATTGAAACCCATCCCTGACCCCCGCACT ATGACCCATTCACAAACATCTAAAGAACTGATCAAAGTAAATTCATCCTCGTACCTGTAAAAAGACCCGC AATAAGAACACGGTGACAAAAAAAAAACAGAAATTGCTGAAAAAACTCAGCAGTCTGGCAGCATCTGTGA AAGAAAGCAGAGTTAACATTTCGGCTCCAGTGAACCTTCTTTAGAATCTTGCTTCTAATTTCCAGCATTC GCAGTTCTCTTTTTGTTTAGGTGTTAAGAACACTGGTTGGACGCGGAGATACAGGATGGGCTATCAACTT GAGGAACCATCCGTGTATCATGGCATCAACAAGTCCGAATTCCTCAAGGGAGGCGCAGTATTGAAATGTC AAAAACGGAACTCAAATCATGAAGTTACAGGAAAATGGAACAATTCAAGACTTGACTGAATTGACTGTAT TCATTCGTGATGGACATGGCCCAATATCCAATCACAGGAGCAACTGCCAGAATGCGATAAGGTGTCCACA TTGAGAGTCCCTGGCCGTTATCTCAATCTTCGTCAGCTTGGTAATGATTTGGTTGGATGATGTGACTGGG TTGTGCGTAAATGCTTGTAAATCTCCTGGGAGCCGTCCTCCTGACGTGGGTTCAGGTTGGGAAAGCGCCT TTAAAAGGAGCTGCCACTTCGAAGAGCCAGTAGGAGCGGAGCTGTCCACTGAGCTGGTGCTGAAACTCGG GCAACTTTCGAAAGCTGTAAAGCCTCTCACTTTCTTCCTTCCCTCGACGAGGTACCTCACTTTGTGCAGT GATGTGGTGCAGCCGAGTTCAGTGCGCCCTCGCCCTGCTCTCCATCGCTCTGGCAGTGCTGAGTGCGGGC $\begin{array}{lllllllllllllllllllllll}M & W & C & S & R & V & Q & C & A & L & A & L & L & S & I & A & L & A & V & L & S & A & G\end{array}$ GCCGCTCCCACCGCCGACAGGTATCGCGAACTCCTCCAGACATCAATGGCTGGCGCAGAGCCCCGGAGTA $\begin{array}{lllllllllllllllllllllll}A & A & P & T & A & D & R & Y & R & E & L & L & Q & T & S & M & A & G & A & E & P & R & S\end{array}$ AAGCGGTAAGAATCCAGCAACGGGAAACCCCCATGTAATAAAGCGGATAATATGGTTATACCATTTAATT K A

GAAATAGAGCCGAATAAAGGGAGAGACTTATTGCTGAATCTAACCTGATTAGAAAGATGTAATGCCCATC AAATGACGGCATGGATGAGTGAGTTATATTTGAGTTCCACAGATCTCTCGGTTTATAAACCGCTCTGAGC GCGCTTGTTTCGACCGAGAGAGATCACTGAGTTCCCCAGCTCCAGGGAGACAGTACTCTCCCTCTCTCCA GTCGATATCGCCACTCCGGTCAGACTGGAAACCAACGGCTTGCAACTTTCCGCAGTTTTCAGGCATCAGT CCCTCCCTGTCTCCGATCCAAACTCATTTCCTCAGATTGAAATGATAATTCTCTGAGCAGCCTCCAATCG СTCATCTAAAATAACTCTAACCTAGCAGCAAAGAGATGTTGATGTAAATGACGTTAACCTTTCAGGTTTA GATGTTCTGTTTATTAGCGGGAAGCTCAGAACCCACATTCACCAGAATGGCGAGAAAGATAAACGCGCTC TGACTCTTTCTACATTGTATCCGCCTTTCGCTAAACCCTAAGCACCTCGATACCGTCTCATCCGGGGTTT CCTCCTTTTCTCCAAAGACCCGCCTATCATGATTAATTAAGCCCCCAAAACATCCACACGGGATATGAAG GGAGCTGCACAATGGATTTGTTACATTGTGATTTAATTGTCGATTGGGAGGTTCACGGCCTGTTGGAGTC TAGAGATAGGAAATGAGGATAATTTCACCCAGTGGGATACAGGGACTGATGGGCTGACACTATAGTAAAG АTTTGCCAAACTATACTTTCAGATCCAGCGTTTAGTGCTTAAATCAGCACCAATTGAGTGGATTTCTCTG СССТААСтАтССССтTTTCTTAATGTAGGAGCTGATCAAATACAATCTCGTCCAATTGCTCGCGGAGCTG $\begin{array}{llllllllllllll}E & L & I & K & Y & N & L & V & Q & L & L & A & E & L\end{array}$ GCAAACGCCGAGAACGAAGTTTTGGATGGGGAGGATTTGCCGCGGATGGCGAAACAGGATGAGGTACGGG

$\begin{array}{lllllllllllllllllllllll}A & \mathrm{~N} & \mathrm{~A} & \mathrm{E} & \mathrm{N} & \mathrm{E} & \mathrm{V} & \mathrm{L} & \mathrm{D} & \mathrm{G} & \mathrm{E} & \mathrm{D} & \mathrm{L} & \mathrm{P} & \mathrm{R} & \mathrm{M} & \mathrm{A} & \mathrm{K} & \mathrm{Q} & \mathrm{D} & \mathrm{E} & \mathrm{V} & \mathrm{R}\end{array}$ TTGATCTGGAACGATCCGCCAACCCCAATTCGCCCCAGAGGGAACGCAAAGCGGGCTGCAAAAACTTCTT $\begin{array}{llllllllllllllllllllllll}\mathrm{V} & \mathrm{D} & \mathrm{L} & \mathrm{E} & \mathrm{R} & \mathrm{S} & \mathrm{A} & \mathrm{N} & \mathrm{P} & \mathrm{N} & \mathrm{S} & \mathrm{P} & \mathrm{Q} & \mathrm{R} & \mathrm{E} & \mathbf{R} & \mathbf{K} & \mathrm{A} & \mathrm{G} & \mathrm{C} & \mathrm{K} & \mathbf{N} & \mathbf{F} & \mathbf{F}\end{array}$ CTGGAAAACCTTCACATCCTGT TAACTCGCTGACACTCCAGAACAACCCGCGGGCCACTGCTTCATCCCA $\begin{array}{lllllllll}W & K & T & F & T & S & C & -\end{array}$

ATCACGTACGGTCAGATGCGGCAGCACCAGAACTGTTAATTAAATAATGTAATTGATTACTTGATTTGAT 
TTATTTGACACAAGAGTTTAAGTTTGAATTGTTCAGCAGACCAGTCCTCATTGAAACTAGGTAGGACCGG TTTGATTAAAGAGGCTCTTTCTCATTGAAATAAAATCTTTGAAAGTGGAATATTGTGTTCGTGTTTTATG

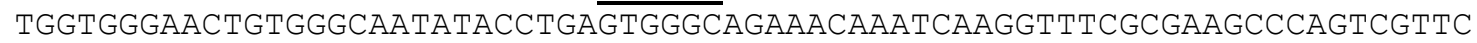
TGGGAAGGTACAATGCGCGGAATTAAGCGCTCACAGTTAGAACAATGTGGCCATCAGCTAGTGGGAATCG CTTTGAGTCCCATTCCCGGAATCTGTTTTGTCTTAAGTTGCTCCGTTACCAAGAGGCAGTGACAATCAGC CTTATCTATATGAAGGGGGTTCTACACCAGGTCTTCGTGCGCATTGTGTCTGTTAGAAGCTATATGGGAG AAGACCCACAGTGCAGGCATCAGGACCCAACCGCTTCAATGTAACCAACAATCCACAAAGCGTAAATTTG CACTCCGCAAACCGGCGGCCAGTCTCTCATTTCAACGGCGGTTTCTCTTAAAACCTGCCATTTGTCTTTG TGCCGTTTGCTGCCCAATCTCAGTGCTGTAGCTGGAGGGAAACATAGTGTTCCAGCTGGGGATAGTGTTG CTGGTGGTGGTGAAGGGATCGGGGCGGGGAGTTGGGGGAGGTGGGTATTGGGAAGGGGATCCATTCCCCA TTCTTCATCTAACAGGATCCAGGAAATAAAAGACACAGGGTGGTTCAATCAGCAAGTGCCCATATCAGTA ATTGCAATCCTGAACTTCAGACAGATCACGGGAGCGCTGACMGTGAGGAAAGCTATCGTTTTACGAAGGG AATAACAACTGATAACAGACTTTACCAAGGGTGTCTGTGTCCCATCAGCAACGCAGACCCGTCCGGGTCA AAGTGAGATGCTAAGCGTGGGAGGGGTTGGTTTCCTCGAGGAAATTTCCGATCAAGCACCCACTTCCTGC СTTAACAAACTAAGGTTCATTATGATATACAGCGGCACGCGGGGAAATAGAACGTACACGAATGCAGAGT TAAAGTGCAACGTCACAACAACACACATGCCTAGGGCACATCTGATCACTATACCGCTGGCAGGAGGGAG AACCACGCACTCCTTAAGAATGCAAAGCATTGTCTTTAGCAAGCCTTCAAGGTGCATCACACCCACATGC TGAGTGTGTACAGACTGACGCTAAATCACCACGGTCAATGACACACTATGGGCCATTAATTTTAGAAGAA TTGTAAACCGCCACAATGTCTAACCTCGTGGCGGAGCGTACCTTCCAGTCTTCCACAATCATCTGTCCAG TCGGCTGTGGAAGAATTATGGGAATGCCATCGCCCTCCCGTTCCTCTCCAAGCTACTCACCATTCTGATT TGGAGACATATTGTTACTGGGTCAAACTGCAAGAACTTCCTTCCAAAGCCATTCTGGATGACCTTACCTC CCAGGAATTTCTGCAGTTGAAAATGGCAGCTCACCACAACGTTCTTCAGAATAATTAGAGGTGGGTTATA AATGTTGGGCCAGCTACAGACATCCACATCCCATCATTCAGCAACAGGAAGGGATATCAGGAATAGCAGC AATCTCATTTAGAAAAGAGACACAGAATTATTCAAGCTGTAACATCGAGATATCTGTCAGAGCCAGCTGA TCAGAACAAAGCACTGTTGTCCTATTATACCCGGTGAAGAATAGCAGTGACTAAGGAGGTAGCGAGAGGA TTAGAATGAAATAACATGAAAATATCCATTCACAATCATGATGAAGTCCGGCTCATGAGTGTTAGGGACA AAGCTGAAAGTTTGTAAAATCTTTTTCTGGAAGTGAACGTGACTATATCTTGCTGAGACTCTCCGAGGAT CCAGTAGGTGAATGAATCATGAGCAAAGAATAGTTCTTTAAGTGTATTGCTGCAAATTCCTCAATTCTGC TGTAAGATCTAATCTCAATTCCCGACTTTTCTAACTATTGACGGACCTTTGTTTTCACATTTCTTCAATC TTTATCTGCGATTTCTTCAATCATTTAATGCTGAGAGAATGCAGATTGCTGTCGACTGAGCAACCAGTAG GAGGTAGAATTCCAGTCTGAACTCACAATTAATGGCAATTTTCCTTCCAAGTGAACAGTACCCTTTATTT TGGACGAGCAACATCCAATGGTTAATAAAAATCGATACATCCAAAAGAAAGGATACGTTTCCCTTATCAT CTGTCACCCCCACATCTTCAATTTGTGATTAAATAAACATGAGGTAGTTCACTCACAAGTAACAAACTCA GAAAGTGATGACATTGGCTGTAAAATGCATTCCTTTTACACTAATCCACATCCAAACTGTGGAAAGCAAG CCATTTACTACCCTGGAAATCTTTTTCTCCTGAGATTCTAAGAAATAGGAACAGGAGTGAGTCAGTCAGT CCTCTGAGCCTGCTCTGCCATTCAACAAAATCTGGGAAGATAAAAAAAAACTGTTTAGCTGATTAAGGTT TGGCTCCATTTAATGTCATTTCATGTACAATTGGATATGGGCAATTCGTAAATCAATGAATTCCCAACAT AGCCCACCTCCACCCCATCTAAATAATGGTGCTTTCCTAACTTTAACCACTGACATGTGGTTCTCTACTC AAGGTACACTGCAGTGTTCCAATATTTTAATCAGTCACCAATTCATTGATTTCAGTGTGATTGCCTGTGT GGCTCAATATTTCACACTTTTCCTGAAACCAATGAAGATTATGAATTCAAGTCTGACTTCAGAGGCTTCA GAATAATACCCAAGCAGGAGCTATTCTTTTGAACCACATTCATTCAAGAAAGCAGCTGGAATTCACTTCC ACTGACTGCAGATATCAAATGAAAAAATGGCGTCAAATTTGCTCATGATCAAAATCTGTCTTTGGTATAC TGAAATAAATATGCCAACTAGATTCAAAACATTTTGATTCAGATTATACTGACCTTAGAGAGCTCATCTG TTGCCTCCCCGCATCAAATCCCAGTGCTCCACTTAATATTCCAAATCACCCCATCTATTAGCCTTGGCAG ATTTTTTAAAAAATAGATCACCTGATAAACCAAAGATTCATCTCCATCTAGTACCTGCCCAAACTTGCTT CTAGGATTATTCAATGTCCTCTGAAATCTAAAGTTCTGATAATGTGCAATGAAATCTAATCAAATATCTT GTAAAACGTACTGCTGGTCTCATCAATTGCCACCAAAAGATAGTCTTGGTTAGTGGAACTTGCAGTTAGA ATCAGTGTAATTTGTAGTTTGGACAGAGTAACTCTTCAGACTTAGTACAACTGAATGCTAGCATAARCTG TTGTCTGTGTAACTCAATACTTACACAATGTGTTGCCAGTGTAATTCTGTGGCATTGTATTTTGGTGTC...

\section{B. Whale shark SST2 (NW_018077477.1)}

...AATGTTGATTCATAACAATTGAACCAGAAAGGAAAAAAGACTGCATTTGAATAGCATCTTTCATGATCT CAATACACCCCAAAGCAACTGAGTTAATTTTAATTTGTTTTTACAATAGTGTAGGAAACATGGTCGGCCA ATTTGTACTATGGAATGTGTTGAACAAGCAGGGATGTTTATTGAGGGATAAATATTGGTCAGGAGAAAGG GGAGGAGAACTCTGCTCTTCTCTTAAATGTTATTTCCACCTCTGAGGGCAGGTTTAACACATTGGTTTAA TGTCTGTTCTGAAACCAGACAAGTTTATAGACTAAATCAATTAAAACCTACCAAAAAATGGCTAAAGTAT GCACCCCATTTGGCAATTCATCAACACACATGAACACGGTTAAATGGATGCCCAAGCAATGTGCAGTGAA TGGTGGGCAGATATCATTATATCAGCTTGTTGCAATAAATTTGAAAACCTGAAGTAAAAACGAGAAGAAG AATTGATTTTCAGTACAGTATATAGATTAAAATGACAAATCCTTGACCAGTGTTTTAAGAAGAAATTTCC AGGAAACAACTTACCTCAGAACTCTCTAAGGTGCTGACGATCAGCTGTTATGATTAATTCTAGTCAGCCC ATGGGTTTTGATAACGCTTGTACTGCATTAGATGGTCTATCTAACATCTAGCTCCACTTTGAAACAGCTA

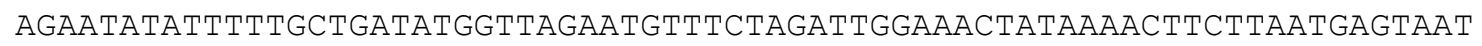


TGTACTTCAATTTTAACCATCCATATTACAGACCTTTCAGACACTTTCATAATAAATTTACAAAACTTAT AGCGGCAATATTATTAGTCAGTTATGTTCTTTGTAAATTAGCATTTTTCCATTTACGATAACACAAACCT GTCGCTCTTTATATACACCGAGATTCCAGGCCTTTATTGGGTTAATGATGCACTTGACTATTAGGCTTTA ATTGTATCATTAATTTGTGCGTCAGAGAGTTGGTTCTGCTGTGCTATAAAAGAACTGGAAAAGCATCCA GTAAAAAAAGGATTGAGGTGTTCACGACACATTGAGCATTTTATTCGGGAGAGCTTGGAGCTTCAGTATG AAGTGGCAA ATGACAGTGTGCCTGATGTCCCTGCTGCTTCTTCTAAGTGTTGAGGCAGCAGACCCACTGG $\begin{array}{llllllllllllllllllll}M & T & V & C & L & M & S & L & L & L & L & L & S & V & E & A & A & D & P & L\end{array}$ AGGAACGAATGAAACTCCAGGTAAATAGGGTGAGTAAAAGGTAATTACGGAATTTGCAAATGTGGCAGGT $\begin{array}{lllllllllll}E & E & R & M & K & L & Q & V & N & R\end{array}$

AGgGGACCAGGCAGAATCAATAATTGTAACAAGCAAAATAGAAAACAGAAGTGTGATGGAAGTGAAAAAA AAGACAGAAGGAAATCCCACTGGAGAGAAGAACAGAACTTTTTTATTTAAAGCAGGTATTGCGGAAGAGA ATTGGCAGTGAATTAAACAGTAATACTGCTGATGCTGGATATCTGAAATATAAACAGAAACTGTTGGGGA AGAAACTTGGCACTGTGGAGAGAGAGGAAAATAGAGTAAATGTTTCACGTTTTAGTTCAGATTTCCAGCA CCTGCAGTATTCTGTTTTTTTAACAGAAAGGAGAGAATTGAAGTAATAGTTTTGGGGAAATTGTAGAGAG AAATGACAGAAGGTGGGATTGTTCTCTAGAACACTGAGAAAGCGGAGGGAAAATTTAATAGAGCTGTTAA AATCTTGAAGAGGGAATAAGTGCAAGTGGTTTCCACTGATAGCAGACCGGTAGCCAGAGGTCACAATTTT AAGTTGgTTTAGAGAGAGAATTTTTTTTtGCACAGTGCATTGTTATAATCTGGAATATGGTGCCTAAAA GTTGAGGGAATGAGATTCAGCAGTAACTTTTAGAAGGAAATTTGATATATAAAGGATGGTAAATTGCACG GCTGTGGGGAAGAAACAGGGAAATGGGACTAACTGGCCAGCTATTCCACAAGGACTAGTACAGGGATGAT TGAATGAATGGTGTTGTTCTTTTCTGTAGGATTCTATGAAAGCCAACATTGGCATCAAGAGATATTGAGT ACATTAgTTATCTCTCAGGACTGGTATTATATAGGTCTGAGTTAATAAACCAGAGAATTTGAGTTCAAAG ACCACTTATTTGAGAACTTGAAATTGTTTGAAAACAAAATGCAGAAACACAAAGCTGGTTCCAGCAAAA GTAAGCGTGAAGCTGTCAGACCGTAATAAATCCCCAACTGGTTCACAAATTTCCTTTAAGGAAAGAAGTT GATCTGATACATGGCCCACAGCTGCTCCAGAGCCAATCTGGATGGCTATTAACTGCCTTGTGAAGTGGTC CAGTAAATCACTCAAATGTACGGGGAGCTGAACAAAAATATAGGCATCTGTTGACATTGAAAACCTACTC AAGATTAGAAAATCCAGTGCGGCTGTGCTTTTGAAAGACTTGTTGATTATACTTAACTAGATAGTGGAAC ATTATTGAAAAGACCAGTAAACCTTAACAACTGAGGGATTTTTGATTTTGCACAAATACTTCGGCAGGAC AGGAGGATCAGGGTTTAAGGAAGTGTTCATTGGCAAAGAGCAGTGAGGAGTTTTATTTATTCATTTTGTG GGATGTAGGCATTACTGCCTGGGCCAGCATTTCTTGCCCATCCCTAGTTGCCCTTAAGAAGGTGGTGGTG AGCTACCTTTTTGAACCGCTGCAGTCCTCCTGCTGTGGGTTGACGCACAATTATGTTAGGGAGGGAATTC CAGGATTTTGACCCAGCAACAGTGAAAGAACGGTGATTTATTTCTAAGTCAGGATAGTTAATGGCTTGGA GGAGAACTTGTGGGTGATGGTGTTCCCATGTATCAGCTGCCCTTGTCCTTCTAGGTGGAAGTGTTTGTGA GTTTGGAAGGAGCTACGTGAGGATCTTTGATTTTCTGCAGTGCATCTTGTAGATAGTACACACTGCTGCA ACTGCAGTAAACTGCAATAAAGCAGGTCGATGGTGGGGAAGGTGCTGCTATAAACTATGAAAAGCATTCA GGACAACTGAGCACAATTTGAGTAAGCAAATGATGCTTGCATCAATGGACAAATGGCTTGTGTTGTATAA AGATAAACAAAAGTTTTTTCTCTCTCTCGgTTTGTCCACAAGGAAGTTACAAAAAGCAGGAAGTATCTG $\begin{array}{llllllllll}\mathrm{E} & \mathrm{V} & \mathrm{T} & \mathrm{K} & \mathrm{S} & \mathrm{R} & \mathrm{K} & \mathrm{Y} & \mathrm{L}\end{array}$ ATGTTAAAACTGCTGTCTGGGCTGTTGGATCCTGATGATAATCTCTTGGAAAATGACTTCACGTCTTTGA $\begin{array}{lllllllllllllllllllllll}M & \mathrm{~L} & \mathrm{~K} & \mathrm{~L} & \mathrm{~L} & \mathrm{~S} & \mathrm{G} & \mathrm{L} & \mathrm{L} & \mathrm{D} & \mathrm{P} & \mathrm{D} & \mathrm{D} & \mathrm{N} & \mathrm{L} & \mathrm{L} & \mathrm{E} & \mathrm{N} & \mathrm{D} & \mathrm{F} & \mathrm{T} & \mathrm{S} & \mathrm{L}\end{array}$ GCCCAGGAGAGGAAGAGAGTTCGCTTGAAGAGAGGTCGGTTAACGCTGGCATCCCTCGTAGACCGCAGAA $\begin{array}{llllllllllllllllllllllll}\mathrm{S} & \mathrm{P} & \mathrm{G} & \mathrm{E} & \mathrm{E} & \mathrm{E} & \mathrm{S} & \mathrm{S} & \mathrm{L} & \mathrm{E} & \mathrm{E} & \mathrm{R} & \mathrm{S} & \mathrm{V} & \mathrm{N} & \mathrm{A} & \mathrm{G} & \mathrm{I} & \mathrm{P} & \mathrm{R} & \mathrm{R} & \mathrm{P} & \mathrm{Q} & \mathbf{K}\end{array}$ GGCACCGTGTAAATTGTCTTCTGGAAAACATTCTCGCACTGTTAATATTGATGGATTTCTAAACCAGT $\begin{array}{lllllllllllllll}A & P & C & K & L & F & F & W & K & T & F & S & H & C & -\end{array}$

AGAACTGTATTTAAACAAAATGGCTTCTGAAGAAGGGTCTAGGCCCAAAATGTCAGCCTTCCTGCTTCTC TCATGCTGCTTGGCCTGCTGTGTTCATCCAGCTCTACACCTTGTTATCTCTGTTTTCCTGTAAAGGCTTA АСTTTAAAATGATCAAAAACTTGCATGCAATTCTGTTCTTTTTCGATATCAATATTTACAACATGTGCCT GCAATGAATTTAAGCGGAGAGCGACCTGTGAAACCCCCTGTCAAATCATGTTTTCGCAAATCAAACCCAG GTTAAACTTTCTAAGTTTTTGTGTTAAGGAAGGTTATTCTTTGGCATGTGATTCCGGGCACTTTCCTGCC GTTTTTCCCCCTCTATTCCCCACCCTAGCATTATTATTTCTTCATCTCCCTGATTTCCCTGTCCCCAAAA GGGTAGTCCTGGATCCAGTTTCCCTCCCTCCCTGATTCAAACCCCATACTGATAGTTTCAGAGAAGGACC TGGACGTAGAGATTCTCTGTCTCACACACACAGTCTCTCAAGTCTGTCAAACTGAGGAGACAGTGTCATT TGGATTTGATGTGATACATGAATGTATAGAACATACAGTCTATCTGCATTGAATGTATATACTTACCAA GCAGTGTGATGCCAACAATAAAAGTATACATTCACACCTGTGTATTAAGCATTGTTATTCTTTTCTATCT CAAACAAAAAGGTAAA $\overline{\text { CAAAAA }}$ ACTCTGCTCCTTCTGTCTTCCTTGTGTTGAATTTGCAGCACCAACAG AACCGGTCTTTAACCCTTTTATTTATTGATACCAATGTTTCAGCTCTCACCGTCAATTACTCACAACCGT TACAGTTTTGCTATCCTAGGGGTGAGGTTGCACAAGCCAGGTGTGTGTGCTCATTGGGCTGTGTGGATTC CGGGGTGATCTGATTTTGCTGATTACAGGGTCCGATAGAATGCACTCTGTCTGAAATTAACTTTCGAGCC AATCTGTTCAgGGTTGACGCCACAAAGAGCTAATCCACACAAAGGTCAATGAGAGGCAATTTAAAATCCC СССТСАСТСССТАСтTTTGACССTCCTCCGCCCACAACACAAAAAAAATTACAAAAAGAAGTGGAAGG GTGATCTAATCTTTAAAACGGTCAGTGGTTTTGATGGAATGAATACACAGATTAAAAATAATCTTAAAAA TATCTTAAAACTAAACAACTTCTTTTCAGAATATGCCGAAGAATCGAGCAAGTACTGTTTGTAGTGTTAA 
ACAGTCATTACTTGCAGAGCAACAGTATTCAAACTAGGATACAAACTGGAAATTCATGATGAGCTGATAC TAGAGATCTTCAAGATTGATTTTGTTTTGACCTGCTGGTTGTCATTTCTGCTGCTGTGAGATAGTTAAGT GTTTGTCCTTGCAAATCATCTCCCTGTCTTTTGGAAGTAAGGACAGGGGCTCCTGGCAATATGTCGATT...

\section{Whale shark SST6 (NW_018032490.1)}

...CCTTGGCATAGGAAAGGGAGAATGTACTTATTTCCAGGTTCCTAACTGCTACACAGTAATGCCTAGTG AGgCATCGGGCACCAGCTGTAATGCTGGCATAGTTGAATAATTTGCTAGCATCCATCACCCAGACTCATA TGTGAAAAAGGGGCACTTGGAAGGAATACTGCAGTACAATCTCCTTGTATGAAACTGTTCTCGCCAAGGA TAAAACATGTCCAGGAAAGGCAGGTGAACAAAAAGGGAGTAGGGAACCTGTTTAATTTGTGAAAACAAAA CAATCCAATTCACTGGAAATCAGTTTTTGACACAAATATACCATCAGATTTAGCATGTTTTAAAAAATTC AGTCATCAAATAATATCCCACAAATGTCATGAGGAAGATCTTTTGATCATATTTTAAAGACCTTATTAGA TATAGTGACAGTCATTTACATTTGCTCAATCTTTCAAAAATCCTTAGCACAAAGAAAAATTCTCACGGAG AAATGACCATCACTCCCTGAACATTTTAACGTTTTTCCCGAAGAAAAGGATCCTGCCTTAAAACTGCAGA CAGAAATTCAACATTGAATTTAATTTTGAATTGAAGACTTCCTCCTCAGTGCTTCTGCAGGCAGAGAATT GCACTATTTCAACATATCTGATTGGATGACCTGGTTCTAAACTAAATCTGGTCAGTGAATGGTTAATCTT GGACTGGGTGTAATTGTGTGTCTTTGATGGAGTCTTAATGTTTAATGAAGCTTTGGTCTGTGCGTCAGTG AGTGGGGCTGTACTGGGTTATAAAAGGGgGTTGGAGACTGTCAAAACCACACAGAGACTTCAAGGGTCGA GGCACATTGCAAAGATCTCGAAAAGGCACCACTTCAATCCAATTCCAAGCGACTGAGAGAAGAGGCAGCA TGCAGCTACTGGGGTCAGCGAGCTTKGTGTCTATACTGCTCATTGTGTCCAGTGTGAAGGCCACAGCACC $\begin{array}{lllllllllllllllllllllllll}M & Q & L & L & G & S & A & S & X & V & S & I & L & L & I & V & S & S & V & K & A & T & A & P\end{array}$ TTTGGAAGACAGACTGAGTCTTCGGGCAAATCAGGTGAGTAAAGTCTTAAAATGTGGGAGCAGTACGTGA $\begin{array}{llllllllllllllll}L & E & D & R & L & S & L & R & A & N & Q\end{array}$

CCAAAAGATAAATCAGTGGAGAGAATAAGTAAGAACCTTAAAGGAATCAAGGAGTAAGAATCAGTGTGGG AGAGAGGTAGATGAATTTAGTTGGCAATTATAGAAAGTGAAATTCGTAGACAAACAAATGACATACATCG GGGGTGTTGATTGGAGATAAAAAATGTGAATAGTACATGATTTTATTACAGAGCAGTGAAATAATATTTT TAACCCCTTATGCAGAAAGAATTAATCCTTCAGAAGAGGAAACCAGCGAGTGAATCAGATTGAGAGGAGC TGAAAGTAATAGTGAGAGA GAATGGGGCTAAAATGAGAGTACGAGAAAATAACAGGTAAATGGAAGAGTG CAGTAAGAGAGTGGGCATGTTAAAATGGGAGGGAACAAGTGCATGAGACAGAAAAAAAACAAAAAACGAA TAGAAAGGGACAATAGAAAAAAATAGAAGACGAGAGAGGAAGTAGTGGAAATAGTAAAAATTAATTGACA AATGTTAAAGAGAGAAAGACAAAACTGMGgGTAAGGATGCACATCAGAAACTGAGAATGACAGAGTGAGA TGGTGAACAAGGGGCAGAAGCGCAGTGTAGGGGAATCAAAAACTGCTTTTACACAGGAGCTAGTTTTGTT TGGATGTCAGAGACTGATTTGCATGACGAGTCTCTTGTGTTTTATTCCCCAATAGTTATCGCCGTGGTAA GTTTGTAAACCCTCAAAAACTTCAGCGCACAAGCCAGGCTCTGGAATAAGACAACCAGAATTAATAGTGC AAATGTCTAGGCCCGAAACGTCAGCTTTGCTGCTCCTCTGATGCTGCTTGACCTGCTGTGTTCATCCAGC TCTACACCTTGTTATCTCTTAATAGTGCAAATACCATGTTCACATGAGCAGTTTCTCAGAATTCGACTTA AACCTGCGTCTCTATAGAAGTGGGAAAAGAATGAGAGTGAAAGAGATCAGAAGACAGAAAGAGCAAATTG AACAGATAAGCTGGGAGTAAGTGAAAATAACCCTTTCATTCCCTCACACAGAATATCTTTTGAAAAGTAG TAAGGCTTCACAGTAAGTAGGAGAGTGAGTGACAGAGACAAAAAAAAACCTGAAAGGCAGATGGAGAACT AATAAGCAGAGAGACTTGGCTCTGACAAGCAATAACAGTGCAAACTAYATGGTCTGACCTCCCTGGTGCA CAATCTCTGCAAAGACTGCCCCTTGTTATGAGACTAAGTCACTTCAGTCACTGTTAGTGTCACGGCAGCT TACTCACCAGCTCTGCTCATTGATGCCACACCATTCCCCTTATTGTTCAGTTCTCAGTGCCGTGTAGCTA AAAGTGATTCCCAAACTACAAATTACTGTCAGTGTTGTAATTGTACGAACGTTTCATGCACTCATATCTG ACTCTCCTCTGGCTCAGCAGAGCTGTTAAAATGTTAATAATACAACAATGTGAATGCAAGTCTGGACTTC CAAGTACAGGTTCTAGTTTTATAGTGTGTATTCCACATTAATTTCCCTTTGCTGTTATCTCTAACAGG TGCTCCTGTCCTAATAAATCGATCGCATTGTCGTATGCATTTCTATTTAATTATAAAGGAGCAAGGAAAC CAAGTGCAGGACTCTCCCTGTTGCTGAGTTGAAGGGAACAGTCCCTGGCAGATGTTACAACTTTGTATTG GATACATGGCTAAGGAGAGATTTAAAGATAAGGGACAGTTCTGCTGTTGAGATCAGTCTTTATTAAATAT AтTAтGCTTATTACATGCTGATTTCCATTTATATTATCAATGGTCAGCATAGCTAGgTCCACACAGTCAC CTGTTTTCATCTTATATCTCAAGATGAGTGGAAGAGTGAGCCTTCATAGAACCATTTGCTCCAGCTTTGG GTGACATATTTACAGATTCTGTGCATCAGTTCAAAGGCTCATCAATCAGACTTGCTTTTGATGAACCTTT GTGTCTATCAAAATGACCAAAAATTAATAGTGTTCTCATTTTAAGGGAGCAATGTCCTCTCACAGGTCAG GTCTGCCACAGATACAGCTCCTTCTGTGCTGTCCCAACAATATACCTTTAACTTCAGTCCCCTCGGTCAA AACAGGCACGAGAGTAAAGATTTCCTTTTTCACTCCAGTCATTCCTGTCATCTCTCTGAGTGTCCAATTG CTGCTAAGGTGGKTCGGTATGTGAGTCTCTGAAAAGCATTGGCTGAGCCTTTTGTTTAGCAGAAGCTAAC AAGATTGAAAAGCTGATTATCCTGTCGTTTTAAAACATGCTTGTTAGGCCATTAATTTAATATTTTACAA GATGGAGTTATTAAATCCATTGGAATATTGGGTATCATTTTTGGAGGTTGAGCATATCTTGAAGCACTGT AATGTCAGGTAATGAGCTCAACTTCACTCTAACTCTAGGATTTTGGCAGCAGGAAATTTCCCATCTCGTA CAAACCTATTTTCTGTACGAGGGGCAGGAAACAGCAAGGGAGTTTCGGTGAGGGCTGGGGGTGGGGGGTG CGGTGGTGGGCAGTGGTGCGTGGGATGTGGGGTAAGGGAATTAAGAGGCAGAGTGGGGACTGTGACTGGG ACTGACTCCACTCCAGAAGTGACTTTGCGTCTGTCATAGATAACAAGGTGTAGAGCTGGATGAACACAGC AGGCCAAGCAGCATCAGGGGAGCAGGGGTCTCGACCTGAAACGTCAGCCTTCCTGCTCCTCTGATGCTGC TTGGCCTGCCGTGTTCATCCAGCTCTACACCTTGTTATCTCAGATTCTCCAGCATCGGCAGTTCTGACTA 
TCTCTTGCATCTGTCATAAGTTGCTGGCCAATCAGATTCCTTGCTAAAGGGGAATGTGCTGCACTGGGGA AACAGATAAATGAGGCAAGTGGACGAATCACATTGTTTCTACAGAGAGTAGAGGGAAGCAAAGATGCATG ATCTTTGTGACCTTAAAAAATCTTTCTCCCCAGTTATTCCACCTCAAGATATTTGCCACTAAATAAGACT TAAGACCGACATTAATGTGCAGAAGGAATAAATAGTGGTGGTGATTATCCACCCAAGGGCAGGACTCCGG СTTATTTCTCTTTGCTTTGTGGTACTGAGGTGTCCTGCCTGGTTGGCAATCTGACTAGGCAAGAGGGGAC ATGAGGTAGTTTCCTCCCATCTTCCTTGTGTGCTTAAAGGAAATGCAGATGCTCTCCTGCATCCCTTGCC TGTAAGCAGCTTGTTTCTAGAGGTTCTACCAACATTACCAACACTGGCTTACAGCCTTCAGCATGGCACC CTCAGCTGGGCCTGGCTCCGTCTCAAGAAAGGATAGGAAAAATCTGTCCCTAAAACCTCAAATGACCAGT AAATAACGTTGCTGAGATTATATTCCACTGGGCTAAAGGCTAAATGTTTCATAGATACCACTGAACAATC CCCCTTTCAGACTAAAACTCAGTCATTCCTTGGTTTGTTATAATATGCAGAATTTTACAGTTTCGGCATG TGACTGTCTTTTGTTGCAAATTGGCCTTGCTCCAAGTTTTCTCTGGCAGTGGTTAGAGGGGCAGCACGGT GGGTCAGTGGTTAACACTGCTGCCTCACAATGCCATGGGGCCTGGGTTTGATTTCACCGTTGAGCGACTG TCAGTGTGGAGTTTGCATATTCTCCCCGTGTCTGCTTGGGTTTCCTCCTGGTGCTCCAGTTTCCTCCCAC ATTCAAAGATGTGGATTGGCCTTGCTAAATTGCCCACAGTGTCCAGTGATGTGTAGGTTAGGTGGATTAG CCATGAGATATGCAGGATTACAGGAATAAGGTAGGGGTTGGGTCTGGGTGGGTTGCTCTTCGGAGGGCCA TTGTGGGGTCAATGGGCCGAATGGCTTGCTTCCACACAGTGGGGGTTCTGTGTTTTCCCCTCTCTGTTCC TTAAGGTGATGACCAATGGCAAAATGTGGGAACTTCCTAATAACCCACTTGAGTGTGAGGCTGGACTATG AATATCTGATCATGTAAGTATAGGTCTAGAGAGGGGAATAATTACCAATTGAACGTTATCTTATTTTCGC CTGGAGTTCACAAGCATTAATTTTTAGTGGAATGTAAAATATTAATAGACACCCTCTGGTCATTTGCAG CTGGACTGTTTTGTTTGAGGTCAGCTAAAAGCATTCCGAGACTGTGTGGCTCAGGACCACAGCAGAAGGA GTGTCTGATGATTGATTTATGTGATATGTGGCCATCAGCTCTTTTTAGCCCTCAATACCTTGATTCTGTT GCTCTATAAGAAAGTGCTGGGATTGTCATATTTACTGAGCTGTTATTCAGCTAACTTCCAGGCTCCAA TCAACTAGGTAACTGAAGGAAACATTAAATAGCGAGTGCAGGAAGACAGAAGAAATTCTCTCCAACAGCC TGTGTATTATTTTATTAAAATATTGAAATGGCGGAAGGCTTTGTTTAAGCTGAGTGCTATACGACTTTAC ATGGAAGGTAATAATGGTTCATCTTTAATATCCTGCGTGCAGCTTTACTTTGTTGTACGTGTTGGCACCT TTTATACTTGTATCCTGCCTCTGCAGAAAATTACGCTGAGTGCCTGTGGTGAAGTATCTCCGCAGATTAT AAAAATACAATCACAAATGCAGTCATTACATCAGCAACATAAATCTGACAGTATTTTAAATGCTGCTCTG TGAAAGCTTTAAGTGTAGCCAGTTATTTTATTTAGAAAAGGCACTTATTCATGGTTATCGTAGAATCTTA CAGCAGAGGAGGCCATTCAGCCCATTGTGCCTGCAATGGGTTTTAGAAATACCTATCCAATAAGTCCCAT TCCTGGATTTTTCCTACCAGTAAATGAGCTAATCTGAATGTTCCAGATCATTCCTTGGGCTCTGCTGGAT TGGTTAATCTTAGTTGGAGAAGTGGCTTTGATGTTACA GTCGGTTGAACCCATCAGATTAAGGGCTGGGT AACATGGGGAGGCGATGGCCTGTTGGTATTATTGCTGGATTGTTGACCCAGATAATGTTCTGGGGACCCA GGTTTGAATCCTGCTATGGCAGATGGTGAAATTTGAAATCAATAAATATCTGGAATTAAGATGACCATGA ATCAAACAAATCTGGTTCACTAATGTCCTTTAGGGAAGGAAACTGCTGCGTGTACTCCATCTGGCTTGCA TGGCCTGTCTGAATCCAGACCCACAGCAATGTGTTTGATTCTTAACTGCCCTCTGGGCAATTAGGGATGA GCAATAAATGCTGCCTGGCCATTGACGCCCTCATCCCATGAATGAATGAAGGAAAAAAAAATCCTGGTTA TCATTCTGCAACACTTGTAGAGAAGTCTCCGTGAATAAAGAGTCTGCTGACTCTCACTCTCTGGACTGAA ACACTCAGCTGATGTTTTAGAAGATAGCATGTACTGAGTAAACTATTCAACCAGCAAGAGATGCCGCGTC TTGTGCAGGAGTGGGACGGGGGGATTGGGGTAATGGGAACAGAAAAACAATGTGACACTCAGCACTGTCA TGTCTGAGTCAGAGTATTCAATCCCATTCCACAGAGCTGACCACATAATTTAGGCCAAAAAGTGACTGTG AGGATGTGCTGTCTTGTTTGAGGCATCTTTTCCAAGACAAGATGTCAAACAGAGGCCCTGCCAGATGAAT GTAGACTATCCCACATCAAGTCAGAAATTGCTGGAGAATCTCAGCAGGTCTGGCAGTGTCTAAGAGAGAG AGAAGCAGAGTTAACGTTTCAAGTCCAGTGACCCTTCCTCAGAACAGTAAACTATCCCGTGTGTTTCCTG ACCAATATTTGCCTCTCAATCAACATTACTGTTACTCGGCCCTTCCTGAGTGTAATTTGATTGCTGCATT TCTGAAAAAAAWTTCTTAATTGCGATTAAAGTGCTCTAGAAGAGCAAAGATGCAACATAATTGCAAGTTA TTTCTTTAACCGGTATGTGTACAAAGGCTCAGGTTGGGGAAAAAAAATTACTCTTTTACACATGGCAGGC CACCCATCACGTGTACAAAATAAAACCTCCACCTCAGCCAAATAAATGGGCTCAATCCTTCAGAGTGCAA TTTGGCACAGGTTTTCACAGAGCGGCAAACACGAATAGTTCTGAGGAAGCATCACTGGACCTGAAACGTT TACTCTGATTTCTTACCATAGATAATACCAGACCTTTTCCAGCAATTCCTGTTCTTGTTTCCGATTTACA АстTCCACAGTTCTTTCGgTTTTTGTGAATGAgTTTCGTGTTTGTCTTTAACAGGAGCTGAATAAAgAAA

E L N K E

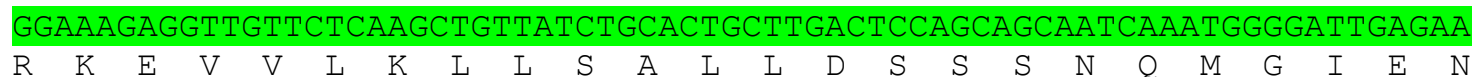
CACGTTCCCAGATCAGGAGGACATGGAAGAGATGAAAGTGGAAGACAGGTCCCGCTTCAGTCAGTTACCA

$\begin{array}{lllllllllllllllllllllll}\mathrm{T} & \mathrm{F} & \mathrm{P} & \mathrm{D} & \mathrm{Q} & \mathrm{E} & \mathrm{D} & \mathrm{M} & \mathrm{E} & \mathrm{E} & \mathrm{M} & \mathrm{K} & \mathrm{V} & \mathrm{E} & \mathrm{D} & \mathrm{R} & \mathrm{S} & \mathrm{R} & \mathrm{F} & \mathrm{S} & \mathrm{Q} & \mathrm{L} & \mathrm{P}\end{array}$
CAACGAGAGCGGAAGACATCCTGCAAAAACTTCTTCTGGAAAACCTTCACCTCCTGCTAACGTCAGACGA $\begin{array}{llllllllllllllllllll}Q & R & E & R & K & T & S & C & K & N & F & F & W & K & T & F & T & S & C & -\end{array}$

TGACATGTCGCTAGGAACTGATTTAGCACTGTACATAAGCTGGTGTTGTTTATTTTCTTTGTTTCTTGGG GGTTACATTTCTTTCATGCACAAGAAAAATTTAAAAAAAAAGCATGAATGTGTGTAATTTTGCTGCAACT TGTATGTATTTCTTTTCCAGCAATGTGGTGCCGCTCGTTCTGTTAGAGCAATAAAGTGGATCCGAAATAA TCAAATTTGATTTGATCGTTTACTGACTTGGCCTTTCCAAAGCTCTGTTTGAGATCTGTTCAGGGGATTG 
ATGTCTTAAGTGAGAGAGGGAAGTCATAGTCAAAGCTACAGGGGAGGCAATGGCCTGGTGGTATTATCRG TGGGCTGTTAATCCAGAGATCCAGGTAATATTTTGGGGACCCGGGTTTGAATCCTGCCATGGCAGATGGT GGAATTTGAATTCAGTATAAAGAAAAATCTAGCTGGTGCTCTGGTTTCCTCCCACAGCCCAAAGATGTGC AGGCTAGGTGGATTGGCCATGCTAAATTGCCTATAGTGTTCAGGGGTGTGTAGATTAGGTCGGTTATAG... 


\section{Figure S6}

\section{A. Whale shark SST1 (SSTa)}

atgtggtgcagccgagttcagtgcgccctcgccctgctctccatcgctctggcagtgctg $\begin{array}{llllllllllllllllllll}M & W & C & S & R & V & Q & C & A & L & A & L & L & S & I & A & I & A & V & L\end{array}$ agtgcgggcgccgctcccaccgccgacaggtatcgcgaactcctccagacatcaatggct

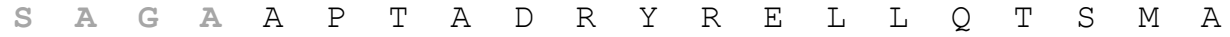
ggcgcagagccccggagtaaagcggagctgatcaaatacaatctcgtccaattgctcgcg $\begin{array}{lllllllllllllllllllll}G & A & E & P & R & S & K & A & E & L & I & K & Y & N & L & V & Q & L & L & A\end{array}$ gagctggcaaacgccgagaacgaagttttggatggggaggatttgccgcggatggcgaaa $\begin{array}{llllllllllllllllllll}E & L & A & N & A & E & N & E & V & L & D & G & E & D & L & P & R & M & A & K\end{array}$ caggatgaggtacgggttgatctggaacgatccgccaaccccaattcgccccagagggaa $\begin{array}{llllllllllllllllllll}Q & D & E & V & R & V & D & L & E & R & S & A & N & P & N & S & P & Q & R & E\end{array}$ cgcaaagcgggctgcaaaacttcttctggaaaccttcacatcctgttaa

$\begin{array}{lllllllllllllllll}\mathbf{R} & \mathrm{K} & \mathrm{A} & \mathrm{G} & \mathrm{C} & \mathrm{K} & \mathrm{N} & \mathrm{F} & \mathrm{F} & \mathbf{W} & \mathrm{K} & \mathrm{T} & \mathrm{F} & \mathrm{T} & \mathrm{S} & \mathrm{C} & -\end{array}$

\section{B. Whale shark SST2 (SSTd)}

gaagtggcaa atgacagtgtgcctgatgtccetgctgcttcttctaagtgttgaggcagcagacccactg

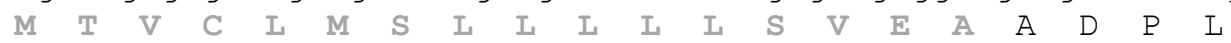
gaggaacgaatgaaactccaggtaaatagggaagttacaaaaagcaggaagtatctgatg $\begin{array}{llllllllllllllllllllll}E & E & R & M & K & L & Q & V & N & R & E & V & T & K & S & R & K & Y & L & M\end{array}$ ttaaactgctgtctgggctgttggatcctgatgataatctcttggaaaatgacttcacg $\begin{array}{llllllllllllllllllll}\mathrm{L} & \mathrm{K} & \mathrm{L} & \mathrm{L} & \mathrm{S} & \mathrm{G} & \mathrm{L} & \mathrm{L} & \mathrm{D} & \mathrm{P} & \mathrm{D} & \mathrm{D} & \mathrm{N} & \mathrm{L} & \mathrm{L} & \mathrm{E} & \mathrm{N} & \mathrm{D} & \mathrm{F} & \mathrm{T}\end{array}$ tctttgagcccaggagaggaagagagttcgcttgaagagaggtcggttaacgctggcatc $\begin{array}{llllllllllllllllllll}S & L & S & P & G & E & E & E & S & S & L & E & E & R & S & V & N & A & G & I\end{array}$ cctcgtagaccgcagaaggcaccgtgtaaattgttcttctggaaacattctcgcactgt $\begin{array}{llllllllllllllllllll}\mathrm{P} & \mathrm{R} & \mathrm{R} & \mathrm{P} & \mathrm{Q} & \mathrm{K} & \mathrm{A} & \mathrm{P} & \mathrm{C} & \mathrm{K} & \mathrm{L} & \mathrm{F} & \mathrm{F} & \mathrm{W} & \mathrm{K} & \mathrm{T} & \mathrm{F} & \mathrm{S} & \mathrm{H} & \mathrm{C}\end{array}$ taattat

\section{Whale shark SST3 (SSTe) (exon 2 only)}

gactttctgaaggacacaattatccaacttctacatgtggtcgcggatgcagacaaggat $\begin{array}{llllllllllllllllllll}D & F & L & K & D & T & I & I & Q & L & L & H & V & V & A & D & A & D & K & D\end{array}$ gctgtggaagtgaatgcttctcaagttgagactccaccagwtaacaagattccaatgaaa $\begin{array}{lllllllllllllllllllll}A & V & E & V & N & A & S & Q & V & E & T & P & P & X & N & K & I & P & M & K\end{array}$ ttccagcagcgtcaattgggaacaagaatgcggaaaactgcaagaatttcttctggaaa $\begin{array}{llllllllllllllllllll}\mathrm{F} & \mathrm{Q} & \mathrm{Q} & \mathrm{R} & \mathrm{Q} & \mathrm{L} & \mathrm{G} & \mathrm{T} & \mathrm{R} & \mathrm{M} & \mathbf{R} & \mathbf{K} & \mathbf{N} & \mathbf{C} & \mathbf{K} & \mathbf{N} & \mathbf{F} & \mathbf{F} & \mathbf{W} & \mathbf{K}\end{array}$ acatacacttatgttaa

T Y T I C -

\section{Whale shark SST6 (SSTc)}

aaccacacagagacttcaagggtcgaggcacattg caaagatctcgaaaaggcaccacttcaatccaattccaagcgactgagagaagaggcagc atgcagctactggggtcagcgagcttkgtgtctatactgctcattgtgtccagtgtgaag

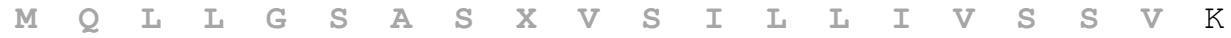
gccacagcacctttggaagacagactgagtcttcgggcaaatcaggagctgaataaagaa $\begin{array}{llllllllllllllllllll}A & T & A & P & L & E & D & R & L & S & L & R & A & N & Q & E & L & N & K & E\end{array}$ aggaaagaggttgttctcaagctgttatctgcactgcttgactccagcagcaatcaatg $\begin{array}{llllllllllllllllllll}R & K & E & V & V & L & K & L & L & S & A & L & L & D & S & S & S & N & Q & M\end{array}$ gggattgagaacacgttcccagatcaggaggacatggaagagatgaaagtggaagacagg $\begin{array}{llllllllllllllllllllll}G & I & E & N & T & F & P & D & Q & E & D & M & E & E & M & K & V & E & D & R\end{array}$ tcccgcttcagtcagttaccacaacgagagcggaagacatcctgcaaaacttcttctgg

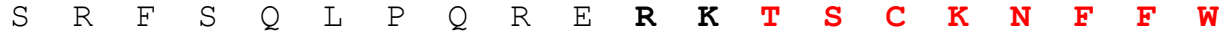
aaaaccttcacctcctgctaacgtcagacgatgacatgtcgctaggaactgatttagcac $\begin{array}{lllllll}\mathrm{K} & \mathrm{T} & \mathrm{F} & \mathrm{T} & \mathrm{S} & \mathrm{C} & -\end{array}$ 
tgtacataagctggtgttgtttattttctttgtttcttgggggttacatttctttcatgc acaagaaa 


\section{Figure S7}

\section{A. Elephant shark SST1 (Sc_821)}

...TTTAGTGGATGGTATGTTGTGGATGCTGTATAGGGGATTGTGTGTAATGGATTGTGTGTAAGGGATGGT GTGTAGTGGTTGGTGTGTAGAGGATAGTGTGTAGTGGATGGTGTGTGGTGGTTGGTTTGTAGAGGATAGT GTATAGTGGACGGTGTGTGGTGGTTGGTGCGTTGGGCATGGTGTGCGGAGGATGGTGTTCGCGATGGTGT GTAGAAGACTGTTTGTAGAGGATGCTGTGCAGTGAAGGGTGTGTTGGGGATGGTATGTAGTGAATGGTGT GTAATGGATAGTGTTAAGGGATGGTGTATATGGGATGGTGTGTAGAGGATGGTGTGTTGGGGATGGTGTG TTCAGGATGTCCTTCTAATATTTTGCACTACAGTATTAGAATTGAAAAGTTTGCCTCTTGTTTTTGCTCT GTATTTAAATCCATAGAATTAATAAGAAAAAAAATGTAATATTTAAGAGGAGGTGGACGTGGTACGAATC TCTGCCCTAATCTTAAAAGCCTCGTCACGGCCGGATGGGTGGGGGTGGGAGAGTCAACGTATCGGAGGCG ATAACATCCCCAAGTGGCGAGGTTATGGACATTATCTCAATACTCGTCACGTGGGTTATTATTTTGTTCG ATGATGTGACTAAATTGAGCATAAATTGCTTAAATCTCTGAGGACATTTGCTTTTGACGTCAGAAGGTGG CGTGAGGCGGCTTTATAAGGCGAGTCTATGGACACCAGGACCAGAAGATCCAGACGAGGCAGAGCGTCCA GAGAGCCGGTGCTGAACCACGAGCGTTCCTCCGTTCCTCTCTCGCTCCTCCTTTGACAGTTTGGTGCCGT CGCCCCGA ATGTCGTGCGGCCGAGTGCAGTGCGCCCTGGCGTTGCTCTCCATCGCCCTGACAGTGCTGA

$$
\begin{array}{llllllllllllllllllll}
M & S & C & G & R & V & Q & C & A & L & A & L & L & S & I & A & L & T & V & L
\end{array}
$$

GTGTCACCTCGGCGCCAGCACACGACAGGTACCGCGAAATCCTGCAGCGATCACTGGCCGCGGCGGGAGC $\begin{array}{llllllllllllllllllllllll}S & V & T & S & A & P & A & H & D & R & Y & R & E & I & L & Q & R & S & L & A & A & A & G & A\end{array}$ CAGGAGTAAACCGGTAAGACAGCCGATTCCACACTGGGTAATTAATATCTGACAAGGTTGCTAAATACAC R $\quad S \quad K \quad P$

ATATTTACAACATTGACCTGTATTTCTAAAGTACATTCATTTGGGATTTTTGACTGGAGCTAATTATCCG ACCGTCAGATTTTATTTCCTCATTTCTGTAATTCTGAATATAGCGCATTTCAGATGTCAGTCTATAACTG TAAAGGAGATATCACGGTAACTCGTCTCTAACTTGCACAGAGGCAGATGCGCACCTGCCAGGATCGCCGA TGGCCCCAGAGACACACCCCGATCTGGAGCCCCGGTACCGCGGCTCTCGGGCTCTGAGGATAACATTCCA AACCATCAGATTACCGCACTCTCAGTCTCAGGGCAACTGCTCAGTGCCGCCGAGGACCCATAGCTCCCGA GCGCTCCGTGTCAGTCGTGTAGTTGGAACCGTTGACACCTTGAGAGCCATTCATTCGTCTAAACCAGTTT TATTTTAATGATTAATGCATCAATTGGCATCAAACGACAATTTTAACTTTCAAGATTTGAGACAGTTTCT AACTGAACTTCCCCCAAAATCTCTTCCGACAATTGTCCCCATAAACAGAAACCTCCCGAACACAGCACAA GAACCAAGTTAAACAGCTACAGATTGTAAGTAGCTGTCAAAACAACCGCGTAACTAGTAACTAGTCCGGT TGGTGCATTTCTGGGCACAATAGAGAGGACTCCAGACTCCAGGCTGAAAACCTTACATCTTTCTTACATT TACTAAAACATTGATATCTCCTAAGCTGAGAGAGCACATCCGTGTTCACCCCCCACCCCCACCCCCACCC CGGAGCGCTGGCCTCTCATGTAGCCAATGGCTTAGAACCTATTTCTCCGGGAAAGTGAATGCTCGTGATT TACATTCCGGTTTCTGAATTGAGTGATTTTCAGACATTTCTTGTCTTAGTGAGTAGTTTGGACTGAGGAG GAGTTTGACGTATATAGGAGGTGGGAGTCACTGAGCCATAAACAGACAAACACGCTGATTTTCCTGATCT TCACACAAACAGCCAAAGCTGGAGAGGAAACTGCGCCAAACGATACATTTTTGTGAATGTAGTCATTAAT CCGGGTTGAGTAATCAGCCATCAGCTGCTTGCCATGTGTACATGTGCCTCACTGATTACTGTTCTCTTGT GTTACTGTTCTCAGGAGCTGACCAAGTACAGCCTGGCTCAGCTGTTGGCGGAATTGGCCAACGCGGAGAA $\begin{array}{lllllllllllllllllll}E & L & T & K & Y & S & L & A & Q & L & L & A & E & L & A & N & A & E & N\end{array}$ CGAGGCGCTGGAGGCGGAGGACATGGCCCGAGCAACCGCACAGGACGAAGTAAGGGTTGAATTGGAGAGA

$\begin{array}{lllllllllllllllllllllll}\mathrm{E} & \mathrm{A} & \mathrm{L} & \mathrm{E} & \mathrm{A} & \mathrm{E} & \mathrm{D} & \mathrm{M} & \mathrm{A} & \mathrm{R} & \mathrm{A} & \mathrm{T} & \mathrm{A} & \mathrm{Q} & \mathrm{D} & \mathrm{E} & \mathrm{V} & \mathrm{R} & \mathrm{V} & \mathrm{E} & \mathrm{L} & \mathrm{E} & \mathrm{R}\end{array}$

TCGGCCAACCCCAACCTGGCGCAGAGAGAGCGCAAAGCGGGCTGTAAAAGCTTCTTCTGGAAAACCTTCA
$\begin{array}{lllllllllllllllllllllll}\mathrm{S} & \mathrm{A} & \mathrm{N} & \mathrm{P} & \mathrm{N} & \mathrm{L} & \mathrm{A} & \mathrm{Q} & \mathrm{R} & \mathrm{E} & \mathbf{R} & \mathbf{K} & \mathrm{A} & \mathrm{G} & \mathrm{C} & \mathrm{K} & \mathbf{S} & \mathbf{F} & \mathbf{F} & \mathrm{W} & \mathrm{K} & \mathrm{T} & \mathbf{F}\end{array}$ CTTCCTGCTAACTCTGAGCAGCCCCCACCACCCCTCTCACACACACTCATACACACACTTTCCCTGACCC T S C -

CCACCCCTCCCATCCCCACCCTACACACAAAGTTTTCCCGATCCCCCGCCACACACACACACACACACAT TGTATTTGTCTTACGCTACG TGGTCTGTTCTGATTTTAACATACTGTAAATAAAACACATGGTGATGATA ATTCAACCTAATTTATTGTGACAGAACCTTTAGATGGGGTTGTGACTGTATAGTTACCCACTCTTTCTGG CTAGAGGTGTATGTATTATTTTTAATTTGTATGTCGCAATGAAATACATGTTTGAAACTGAAACCATCTC TTCTCTTTCTTGCCTGACGTGTGAAAGTCGCTGGTTACCCGGCTCAGCTCCATCACTGACGGCAAAGCGT AGCTGTAACATTACACGAGTTCTATATATTTATATCACTTTCTCAAAACGTAAACCATTGTATCACAGAT GTCTTTTCAGCGATGTGGAGTCCTGTAGCTCAGTGGTTAGAGCACTCGCTTTGCAAGTGAGAGACCTGAG TTCAATCCCCGGGGGGTCAGGGCCAAACCTCGGGCAAGTTTCCTTACTCCACACAGAGCCTAATAATAAT CCTCCAAAACCAAAAACAATAATAAATTGGTCATTTTCAATCTACGACTGTTTGTGGGACATTGCTGTGC GCAATTGGCTGCCGCGTTCGCCCACAAATATACAGTCAATTCACTTTACAGTGTGTTCTGTGAAGTGCTT TGGGACATCCTCCCGACGTGAAAAGCGCTATATCAAATGCAAGGATTATTATTATTATTATGGCTCAGAC AGATCTGCCATAGTCTTTCACTGTGTGATTGACATTTCGGCTCTCATTTCACCAACTCCAGAACAGCTCA CTGGAAGCGATCATTTGAACACAGCAATGACCGGGGGCGACCCCCACCGTCTGTCTCAATGTAATCCTCA CTCTTGTTCTGCTCTCACTCTCGCACTCCTTCCCTCAACCATTCCATCACCAGATGGTTATCTCATACTC... 



\section{B. Elephant shark SST2 (SC_58)}

...TACTTATTTTTTCACATAATAATAATAATCCTTGCATTTGATATAGCGCTTTTCACGTCGGGAGGACA TCCCAAAGCGCTTCACAGAACACACTGTAAAGTGAATTGACTGTATATTTGTGGGCGAACGCGGCAGCCA ATTGCGCACAGCAATGTCCCACAAACAGTCGTAGACTGAAAATAACCAATTTATTATTGTTTTGTTTTTT GGAGGGATTATTGTGGTTGCTTGTGGTTGCCCTGTTGTCAGTTTAATTAGCTTATCTTGTAAAAAAGAAA CAATGAGGTTTTGAATGAAGAAACAATTAACAATCCTGTTTGAGACTGAAATCTTCCGACTTGATATGAC TTGTGTGAGGTTTTTTACTCTATTCATACCCTTGCTGTTCAGTCTAAGTGTTATAACTTAATCGACCTTC TTGCAGTACTTAGATTTTTGTGCACACCTCACCTCATAATAGAAAGAAGCACTTGTAATTTTATAGTACC TTAACATGTTGAAGTGGCTCAAAATGCTTTACGGTATTGAGTGTGCTGTAATGGAAATAATGATAATAGT ACTTGCATTGCACCTTATATTGTCGTTGAGACGTCTCAAAGCAAGCGCTTCACAGACTATAATGTAAAGT GGATTGACTGTAGTTTGTGGTGAAAAGTATCAGAAATCCTGATAACCGTCTACGACAGATGTATGAACAC TATCAACCGAGCTGAATTAATACCCATTAATGTGGGCAAATCACTAACGAGGCCACTTTTTTTAGTCTTT TTGAAACTGATAGTAACCAAAGTCTCAAACTCAGCCTAAGGATAATTTGCCACTGTATAGGCAAAAGGAG CTCTAGTGAGAAAAGTTCACGTTTAGATATTGTTTGGAGGAATGTATTTAAAGTTCTAATAATAAAATTA TGAAACAGCACCTCATGACATCGTACCGACAACTCAAAGCACTTCACAGAATTTTACCGTACAGTGTAGT CAATGTGTGTAGGCAAACGCGACAACCAGAACAAAACGATGTCCCACGAACAGCATGATGTCAATCTAGA CTGAGTACAAAGTCCCAACATGGGGCTTGAACCCATGATCTTCTGACTCAGATGAGAACAGTTAGTGACA TTTTGAAGACAATCTTGTGGTGAGGATGGCGTTTAACCCTTATTTCCTACTTGTCAGGAAAGAGTAAGTG TAAAACGCAAAGGTCAAATTCTTTTAATTTACGTAATGGTACTGATATGTCCCTTGTTAATGTCAGGGCT ATTCAAGTGGAACATTAGgGCATATATCAGACCTTACACGTGACCCACAAACCAGACTATAAACAAGACC TGCTATTTCAATCTTTCCAGTTTCTTCAGCAAAATCGATACGGCCAATTGAGAAATTAAATAACTATAAT TGATGTAGATCACCCAAATCACAACTGTTTGTGGGGCACTGTTGTGCGTAATTGGCTGCCTCGTTTTACC CACAAAATGTACAgTATATTCTGTGAATCACTTTGAGACATCTTGAAGATGTGATAAGgTACTATATCAA ATGCAAGgATTATTATTATATTGATATTGAGACGTCTTCCCGATGTGAAAAGCACTATACGAATGTAATC TTCTTGTGGAAATGTTACAAAACTCAGAGCTGGCTAAATGTTTGTTCTACAGACAAATTCCCCATGAAGA GTCCCAACATCCCATAAGCTGCTGAACCCTTATTTAGTAAACTGGAGGTCAATCATGTAATACTGTGGAC СTTTCTGGCTCATGTATCAGATCGCCCAACGAGAGTCTATCAAAGAGAATTGTCTCAGCTCCACTAGTCA TGTTAGATTGGTGTTGGCATCACCCCTTTCTCAGAGTTGTCCCATGACATCAATTCTCTCCGCAAACTCA GGCTTCAGCCTCACTGATTCCTTATCAGTCTTTGAAAACAATGACTTTCTGAGTTGTGAAAATCTTATGT TCTGTAACAGGCATTATTTCCTGTTAGGGTCATCAAGTGGTATTGCCAAGGGGAGCCACTTTTTTTAATA GATCAGATGACAGATTGGTTTAATGTCTCATTTGAAGGACAGATGATTCATGGTAAGACGAAAAAGTTTC ATTTCTTGTGGATTTCTAGAGCTGGAACAATTTCTACCTCCACTTTAATTCGTAGAAGTGTTTTTTGTGT GTTGGATATTATAGAAGATTGATGTTGAAAGCTCTATACAAAACACACAAAGACTGACTCATTCTAAATT GGAAAGCTCTTTTAATTATCAGTGTTCTAATCTTTGATGCAAATCGAAATTGGAAAATATAAAATAAATC AАTTATGATCTTTCTAAATTAACATTTTTTTCCAGTTGTTGACTTTGTCATTTTTTTAATGTTCCAGTTA ATGGTTCTCATTGTATATTAGTGTCTGATTATAGCACTAATTTTGCACGTCAGCGAGTGCTATAATTGTG CTGCATTATAAAAGCAGGACAATTAGAGTTTTCTCAGCAAAACAGGGAAAGAGTCAGTGAAAGCTCGACA GATCCATTGAAAAGAAGAAATTTGAGTCCAGCATGCAGTGGCAAGCATTCGCAAGTGTCGTGTGTCTG $\begin{array}{lllllllllllll}M & Q & W & Q & A & F & A & S & V & V & C & L\end{array}$ TTGTTCATTGTAACTGTTCAGGGAGTTGAACCACAGGAGGAACGAATGGGTCTTCAGCTGAACAGGGTGA $\begin{array}{llllllllllllllllllllll}\mathrm{L} & \mathrm{F} & \mathrm{I} & \mathrm{V} & \mathrm{T} & \mathrm{V} & \mathrm{Q} & \mathrm{G} & \mathrm{V} & \mathrm{E} & \mathrm{P} & \mathrm{Q} & \mathrm{E} & \mathrm{E} & \mathrm{R} & \mathrm{M} & \mathrm{G} & \mathrm{L} & \mathrm{Q} & \mathrm{L} & \mathrm{N} & \mathrm{R}\end{array}$ GTAAAATAATAATAATCCTTGCATTTGATATCGCGCATTATCACGTCGTTGATATGTCTCAACGCCCTTC ACAGAATGTACTGTAAAGTGGTGACTATTTGGGGCCAAATTCGGCAGTCAGTTGCGCACAGCTGTGTCCC ACCATCAGTCGTGGATTTAAACTCGCATGAATTTTACCAAAAAAAAAAAGTTTCTTCTCTCCATCCAACA GGAGATGGTAAAGGACAAGAAACGCCTGATTGCGAAGCTGCTGGCGGGATTGTTGGACGTGGATGACAAT

$\begin{array}{lllllllllllllllllllllll}E & M & V & K & D & K & K & R & L & I & A & K & L & L & A & G & L & L & D & V & D & D & N\end{array}$ CTGTTAGAAAGTGTCATCGCCCCATTGGGTCTGAGCAAAGAAGAGGGGACTGATTTCGAGGAGAGATCAG

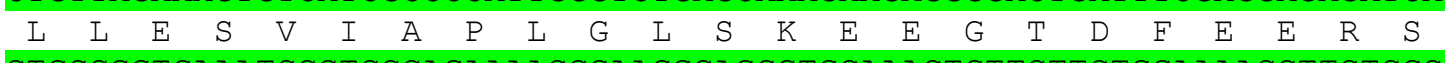

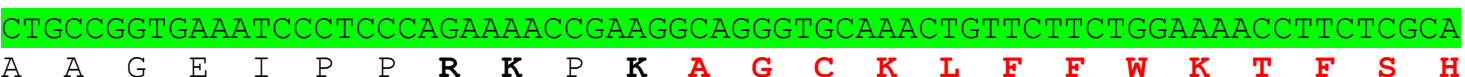
$\begin{array}{llllllllllllllllllllllll}\mathrm{A} & \mathrm{A} & \mathrm{G} & \mathrm{E} & \mathrm{I} & \mathrm{P} & \mathrm{P} & \mathbf{R} & \mathbf{K} & \mathrm{P} & \mathbf{K} & \mathbf{A} & \mathbf{G} & \mathrm{C} & \mathbf{K} & \mathrm{L} & \mathbf{F} & \mathbf{F} & \mathbf{W} & \mathbf{K} & \mathbf{T} & \mathbf{F} & \mathbf{S} & \mathrm{H}\end{array}$

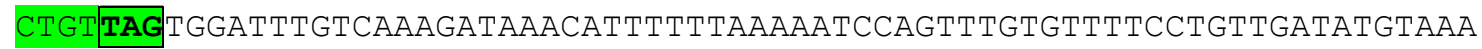
$\mathrm{C}-$

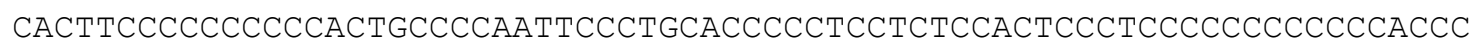
CTTCCATACATCAAACGATTTGTAAATATTACTGAAATAAAACTGGAAAATAAATAGGTGGCTTTCTAA AAGGCGACGTAAAATGATTACATTTTAATAAATGGAAATTCTGCATTAGAATTCACAGTGTCTTGTGCGT TTTTTTAAAGGAAAAGAATCTGAATTATTCCCATGTTCCCCTTTAATCATCCGCTGCGCTAAAGAGATGC ACAGGTCCCTGCTCAGCGCATCCCGCGGCAGTGAAGCTCATATCTTAACTCATACCCACAGCCCACAACT TTCTGACTCAGAGGCAAGAGTGAAACCAACTGAGCCAGACTGACACCTGTGACTAATAGTAATTTCATTT CAGACATCTTATCACATCTTCAAAACATCTCAAAGCGATTCACACGATATACTGTAAAGCGCAGTGACTA TTTTCAAGAGTGTTATTGTTGCATTTGGAAGGGTTGTGTGTCAGCCTGGCTCAGTCGGTAATGTTTTCA... 


\section{Elephant shark SST3 (Sc_821)}

...TGGGCAGGGCATTTGGGAATAAATTAATCAAATTATTAATCAGGGTGGGTAGGGCATTGGGATTAAATTA GACATCTTGCTCTGACAGCCTGTACAGAATCAATAAACTGAATGGACTCCTTCTGCACTGTAAATTTCTG TGTCTCTGAGTTGAGTCAGTCGGTGAGATTTTTGAAGCGTTTCCAATCCCACGTAATTGTGTATCATTAA TTATATATATTATATATGCTTACATGTGTCTAACATTTTTGTGTGGGTGTGTATGAGAGATATGCATGTA TTGTATAGCACCTCATGGATAGTACTCGTTATGCCGCTACACATGGATAAACTGAAGTTTGCTTTGAGTG TCCATCAACCTTAATCTGTCTGGACAGGTGGCTGTTTGCAGATGAATTATAAAAATCTGTCCACCTTCCT GGGTAAATATTTGCTAATTAGTTGTGTTTGCAATAAGTGAGTGGATCAGCTGAAACCAGAAAGCCATTAT CACATTTTCACCTGAAATTAAAATTGCTCAGTGAACCACTCAGCCCTACCACATGGGAGCTGTGTCCAGC ATTCACGGGGGGAGGGGGCAGCTAGTGAGGGCTACTGATTGCACACACAGTCAAGGGAAGAAACACTCAC TGGCAGCTGCTGATGGACATTCATTGTGCTGATTCCAGTGCTTTTATGCTGGAAATTGGAAGACTTGAGT TGAGTCAGTAAGTGAGGTTAATGGAGTGGCTCCAATCCCATCTAATTGTTTAATGAATAGAGATGGGCTG AGGCAGAAGGTGGGAGTCAAGCGGCTAAATTTGATGGAAGAGCTCTCCATTTAAATTCCAAGCCTCTTGT GTTTAATTGACAGCAGAAATTCACAGACCATTTAAAGCTGTAACTAATCTTTACACCGTTTCACTTCCGC TTTTGACAGAAACAAACATTTACAGTCAAAGCACTAGACCAATTCTCAATACTATTTCCATAACATATTT AACAAAGGCCCAATAACTTTTCCACAACACGCAAGGAGAACATTTATTACATGTGATTTGATAGTTATCA ACAGTGAGACTGTGTGCAATGGGGGAAGTTCTCTGTCTAAGTGAGTGTGGGGTGGGGTGGATATGGGTGT ATGTTTGTGTGTGTTCGGTGGGGGCTGTGATCGGAGGCTGACTGTTGCTTTACCTTATGATCTGGGTTTA AATCTTCAGTGGTAGGGGTGACTGACTGTGAGCACCCTGACAGGGGAGGGAAATTGGGGCCAATCCTAAT AGCTGTGCACAAAGCTGGCCCTGGCCTGGACCTTGCTCAGGCGGTCTCGTGTGGGAAATGGAAGTTCATG GTTGAGAATAGGGAGCCTGAGGGTAATGGAGGAGATACCCTGAGCGATCTAAGCACTGACTGGTTTCCTT GCATTTGATCAGTATCTGTGATGATCAGCCTGACAGAGGAGGGTAATTGTGCATAAATGTCAGGTCACTC TCCCCAGGTGTTATTTAACATCAGGTGATTATGCGAGAGTGGCTTTATAAAGCAGGACATAGGGTACCCC CACAGTGCAGTGATCGGATCAGCATTGCTGACAGTTTGAGACTGACACACATTGCAGACTGTGCTCCGGT GTTGGCAGCA ATGCAGGCTCGGTGCACCCTCACTCTAGCCCTCCTCTCCCTGGCAGCGCTTGGCCTCAGT $\begin{array}{llllllllllllllllllll}M & Q & A & R & C & T & L & T & L & A & L & L & S & L & A & A & L & G & L & S\end{array}$ GCTTTGCCCACACACCTCAGGGACCAGCTCGTTCTCCAGGGATCACCAGCAATGGTAAGTAAGCTTCACC $\begin{array}{lllllllllllllllllll}A & L & P & T & H & L & R & D & Q & L & V & L & Q & G & S & P & A & M\end{array}$

TCACCAGCTTTCAGCCTCTAGGCCTCAACCCAAGCTTGAGTTATTTCTAATTGACAACTTGCCTTCACTC AAGATTAACAGTAAGGATTGTATTAGTGGCTCATAGAATCACAGAATTATACAGCACAGAAGAAGGCCCA TCGTGTCTGTGCTGAATCTTTTAAAAGAGCTATCCAAATCAATCCTACTCCCCCGCTCTCCCTATAGCCC TGCAAATCTGTCCTTTCCAAATAATTATCTATTTCTCTCTTAAAAGTTGTTATTAAATCAGCTTCCACCA GCCTTTCAGGCAATGCATTTCAGATCCAAAATTGAATTGAAAACATCTATTAAGTCTCCTCTTAACCTTC TCTGCTCCAAGGAGAATAATCCCAGCTTCTCTACCTCTCATGATAACTGAAGTCCCTCATTCCTGGTACC ATTCCGGTCAATCTCCTTTACACCCTCTCCAATGCCTTGACATCCTTCCTAAAGTGTGGGGCCCAGAACT GAACCGAGTCTCTCGCTGAGGCCTAACCAGTGTTTTATATAAAGGTTCATCATAACTTCCTGACTTGTGT ATTCTAAGCCCCTATTTATAAAGCCCAGTATCCCGTATGTTTTTTTTTAACAGCTGTATCTACCTGTCCT GCTGCCTTCAAAGATTTCCTTATATGACCCCCAGGTCTCTCTGTTCCTTCACCCCCTTTACGATTCTACC ATCAGCTTATATTGCCTCTCCTCATTCTTCCTCCCCAATTGCATCACTTTGCATTTCTCCACATTAAACT TCATTTGCCACGTGTCTGCCCATGTTACCAGTCTGTCATCTGAAAACTTGGAAATTGTACTCCCTATAGT CCAGGTCATTTATATAGATAAAAAAAACATCAGTGGTCCCAATACTGACCCCCGGGGGGACACCACTGTA CACTTCCCTCCAGTCAGCAAAACAACCGTTCCCCACACTCTCTGCTTTCTGTCTCCCAGTTCTGTATCCG CTGCCACTGTCCCTTTAATACCGGGGATTTTTATTTTGCTTACAAGTCTCTTATGTGTCACTTTATCAAA TGCCTTTTGAAAATCCATATAAACTACATCAACTGCACTACCTTCATCCGCCCTCTCCGTTACTTCATCA AAAACTCTATCAGGTTTGTTAGACATGATTTGCCTTTAACAAATCTGTGCTGGCTGTTGATACTGTGGCA TTGACTGCATCCTTTTATCAAGAAAGTACTATTCAGTACGTCAGCCGTGCCCGCTGCCTCCACAAGAAGA TTTTCCCTTTAGTCCCTTATTGAGCCCACCCTACCTTTGACTAACCTTTTATTCTTTATATGCTTATAAA ATACCTTAGGTTTCCCTTTTATGTTACCTGCTAATCTTTTCTCATACAATCTCTTTGCCCCTTTTGTTTC СтTCTTCAGTTCTTCTCTATACTTTCTATATTCTATTTGATTATCTTGTGTTGTATTGAAGTGTTCACAT GTTCGTATTGAATTGTCAGCCTGTCTCACTCTTGCCTTTGAGCCAGAAATAGAGGGTTAAAGCCTCATGC CAGGGAGCTTAGCACAGAGATTTGATGGAATACAATTTCATCCTGTGATACATAGAATGATATGGAACAG AAACAGATCATTCGGTACAGCTAATCTGTATTTGACATAACTTGCCTTTCAGTGCCAACTCATCCCTGAA ACTGCCCCAGCTTGTAACTACAGTCATTGCTTGAGACAAACCTTCTGGAGCAATGAGCAGCGAGAGAGAT CCCTGTTATTGCAAACGGGAGGATTCTCTGAGCCCAAGAGGAACTCAGTAAAAACATGTCATCCTGTCCG TTACTCAGCATGTGCCTTAACAAAATAAATCTTTTTTTCCTACAGCTGCTAGGCAAATAAAAATGAATCC AАTTTTTTCAACCTAAGGTGAGATAATTGACTCTTTTATTCACTTCCTGCTGTGCAAATATCAAAGTGAA TAGACTGCTGATGTACTCAGGAGATGGCACACATCTCTCCTCTTGTACTTTATAAAAGCAGCCATTAAAC ACTCTGATCACCATTGAATGGAAAGCTCACAATAATTGTCACTGAGAGGTAATAATAATAATAATCCGTG CATTTGATATAGCACTTTTCACGTCTTCCAGATGTCTCAAAGCGCTTCACAGAATAGACTATAAAGTGAA TTGACTGTATATTTGTGGGCGAAACGCAGCAGCCACTTGTGCACAGCAGTGTCCCACAAACGGTCGTGGA TTGGAATGACCAATTATTTTTTTTGAGGAATTATTATTAGGCGTGTGGAGTAAGGAAACTTGCCCAAGG 
TTTTGCCCTGCCGGGAATCGAACTCAGGTCTCTCACTTGTAATCACCACAGTTTTAAATACCCTTTAAAT AAATGCCCATTAAATACCCTTTGAAGGAATATCCTCTAAATACCTCTGAGCAATGGAATACCGGCAGAAA TGCCACAGATGTCCATTTGAAAGGCCAGACCCCTAGTACTGACGAAAGGTCATCGACCTGAAACGTTAAC TTATTTTCTTCTCTCCATAGATACTGCCAGACCTGCTGAGTGTTCCAAGCAATTTCTGTTTTTATCCCAA GGCTCGGTGTTTTGTTGGTGTCAGTCAGAAAGTACTCGTCACAAGTGCGTTGTGTCGGGACTACCATCTC AAATGGTCTAGGCCAGTGATGGCCAACTTGCAGCCTGCGTGACTAATTTACACGTGTGTATTGTAAGTTG GTCATCCCTGGCGAGGCTGACACTGCAAATCAGCCGTCTTTTGGAGAGTTTCAACAGCATAATGATGCCA TGTGTAATAATGGACCTTTTAAGCAGGGATTAATTACATTGTCCTGTGTAAATATCACTTGACAGCAATT AATCATGAAGCATTTGGGAGCGATTTGACGTGATAAGGTGCTATAAAAAAATTGGATTTGACTTGTTGTC ATGTCTAACAAATCTAATAGAATTTTTTGATGAGGTAACGGAGAGGGTGGATAAAGGTAGTGCAGTTGAT GTCGTTTATATGGACTTTCAAAAGGCATTTGACAAAGTGCCACATGAAAGACTTGTAAGAAAGATAGAAG CCAATGGTATAAAAGGGGCAGCGGATCCCGAACGGGCCGGGAGACAGAAAGCAGAGAGTGGTGGTGAACG GCTGCTTTTCTGACTGGAGGGAAGTGTACAGTGGCGTTCCGCAGGAATCGGTATTAGGACCACTGCTCTT CTTAATATATATAAACTTGGACTTGGGTATAGCAAGTACCATTTCCAAGTTTGTGGATGACACAAAGTTA GGGAGGGGGTAAACAGTGAGAAGGACAGGAACCAACTGCAGGAAGACATAGACAGAATGGTACAATGGGC GACACATGGCAGATGAGTTTCAATGTAGAGAAATGTGAGGTAATGCATTTTGGTAGAAAGAATGAGGGGC AATATAgGCGGTAATACAATCTTAAGGGgGTAAAAGAACAGAGAGACCTGGGGGTGCAGGTAAATGAATC CTTGAAGGTGGAGGGACAGGTAGATACAGCTGTAAAAAGGGCATCTGGGATACTGGGCTTTATAAACAGG GGCATAGAGTACAAGAATCAGGAGATTATGATTAACTTATATAAAACACTGGTTAGGCTTCAGTTAGAAT ACTGCGCCCAGTTCTGGGCACCGCACTATAGGAAGGATGTTAAGGCATTGGAAAGGGTACAGAGGAGATT TACGAGGATGGTACCAGGAATGAGGGACCTTGATGATGAGGAAAGGGTAGAGAGGCCGAGATTATTCTCA TTGGAGCAGAGAAGGTTACGAGGACCTGATAGAGGTCTTCAAAATCAAAATCATTTGATAGGTTAGAAAG GGAACAACTGTTTCCACTGGTGGGTGTGTCGGTGACTCGAGGTCACAGATTCAAAATAGTCTCTAAAAGA AGCCGAGGGGAGGGGAGAAGACATTTTTCACGCAGCGAGTTGTTGGAATCTGGAAGCCATTACCTGAAAG GGCGGTCGAGGCCGATTCGATAACTACTTTTAAAAACGCGGTGGACGGGTATTTGAGGAGGACGGGTTTG CAGGTTATGGGGTAAGGGCCGGGAGTGGGATTGACATTAATTGCTCTATTCAAAGAGCCGCACAGACTCG ATGGGCCGAATGGCCTCTTTTTGTGCTGTACGATTCTATGATTCTATGATTCTATGTGCTTGCACTGGAG GGTGTGTGTCAGCAGGCGGCGCTCTGCCCTCGCTGGGTTACTGCTGTTTTTACATTATTATAGACATTGA GAAGCAGATCAGAAATCTCCGCTTATTAAACACTATAGTGAGGACAGAATGCTGACGCGCCTGCTCTTAA ACTCAGATAGGGGTCAGAGAGGTGACACTATGTAATTACAGAGAACAAATGTCAAAATAAACTAGGTTGC CCTATTTAGAATGTTCAGAAAGTTTAGAGTCTAATGTAGCTACTCAGGGTGCAGCAGGGACGGAGGAGA CTGGGAGGGAAAGGATGGATTGTGATAGTGTTTTAATAATAATAATCCCTGCATTTGATATAGTGCCTTA TCACGTCTTTGAGATGTCTCAAAGCGCTTCACAGAATATACTGTAAAGTGAAATAACAGTAGTTGCTGGG AACACACAGCAGATCAGGCTGCATCTAAGGAGAGAgGAAAGTGAGGTCATGTTTCAGGTAACGATGACCT TTCATCAGAACTGGAAAGTGAATTGACTGTATATTTTATACAGTATTTTATGTGACAACACGCTGTATTT CAGCACAATCAGTTGAATCTCAACGACACTGGCTGTTACGTCAGTAATCTCAGCATCTCAGGGGTCAGTG GATGGAGTGCGCAGGAGGGGGACAGTGCTGCGGAGGTCAGAGATTGTGTTCACTCTGTGATTGGCCGATG CTATTCATGTTATGAGCCACTCAGTCTGTCCTGCGTTGTGTGGCCGCCAGCGGGTGACTTTGCTCTCACC GCTTGTTTGCAGGAGCTGCGGGATGGTGTGGTCCGGGAGCTGATACAGGCAGTGGCCGATGCAGAGAGCA $\begin{array}{lllllllllllllllllll}E & L & R & D & G & V & V & R & E & L & I & Q & A & V & A & D & A & E & S\end{array}$

AACCCAGAGTGAGAGGCAGCAGAGGACTTCGTCCAGATGAAAGGCTGAACTACGGCTTGCGCTGGC
$\mathrm{K}$
TCCACGGTGGCGGAAAGATTGTAAAAATTTCTTCTGGAAAACTTACACTTTGTGCTAATCCTGTGCCAGC $\begin{array}{lllllllllllllllllll}\mathrm{P} & \mathrm{R} & \mathrm{W} & \mathbf{R} & \mathbf{K} & \mathrm{D} & \mathbf{C} & \mathbf{K} & \mathbf{N} & \mathbf{F} & \mathbf{F} & \mathrm{W} & \mathrm{K} & \mathrm{T} & \mathbf{Y} & \mathbf{T} & \mathrm{L} & \mathbf{C} & -\end{array}$

GTTAAAGCCTGACTGAACCACAACCGTCTGGCTGGCGAGAGGTCTTAATTAATGTGTAATATGAATTGTT TGCTATTTAGCATGCCTCAATTATTGTTAATCCTTTACTCATTGTGAATTCTTAATCAAGTGTTTACTGT TACGGGGAACAATAAATCATCATCATAGCTATATTCTGCAATGTGTGAAATATTTGTAAACGATTTGGTG

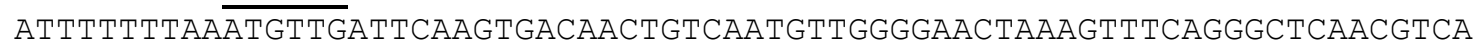
GCTCTGCACTCACACTTCGTATTGAGGCCATTCTGGACTGCAGGAAGGATGTCAAGGCCAAAGGTAGGGC ACATTCACTGGACATCTCATTCTTTCATCCAACAGCCTCCATCACTGCTGCACCCAGACAATAGTCACGG CAGAACCATAGACATTTAAAGCACAGGAGAAGACCATTCTGCCCATAGTCTCTGTGTTCCCCTTAAAGAG CAACCTTTTGGGGCCCATTTATATGCCCATTCCCAAAACCCTGTAATTTAATCTATCTACCATTCCATCT CAACCTGCCTTTCATCTCACTGACCTGATCAAAGTCACAAATAACATTCTCTTTCCTCAGCGTCTTGACC TCTCTGCCACCTACCACACGTAACTCTATCTCTCTGTCTTCACCCTTGATTCCAGGACCACTGTTGAACT GACCGGTTGTCTTTTGGACATCCTGGATGTCCTGGATGAGAGCTAAACTTTCTCTAGTTAAATGTTGACA AGACATCAACACCTTTTCACTTCAGGTACTACAGCATCAATCCACACAGGTGCACTCTCCACCTCATGGA AAGCGTGATGAAAGCATGGATCAGGGTCTCGGGGGCCTGCGGTGAGAGGCAGTGATGGAGGTGGGCAATA CTGCGGAGGTGGTGAAAGCCGGAACGGGTCACAGCACGAATGTGGGGTTTGAAGGATAGCTGAGGGTCGA AAAGGACTCCGAGGTTTTGGATTGACTCAGTGAGGTGAAGAGCACAGCCGTGGGTGGGGATAATGCAGT... 


\section{Elephant shark SST6 (Sc_58)}

...TAATTAATTCATAAATTAATTAAGATGAATTGATCATCCACCTCATGACTGGTGTGTAAGATCTTGCTT TGCACAAAACGGCTGCCACATTTGCCCACGGAATACACAGTCACTACACCTGAGCAGTTGGTGAACTGGT GAATCAAAAGAGGCAGCAAAGATCTTATAGTCAATTCACTTTACAGTGTATTCTGTGAAGTGCTTTGAAA CGTCTTGAAGACGCAATAAGGCACAATATCAAACAGCAATGATTATTATTATTACATTGTGATATTGTAA AGTTTATTTTAATACTAAAGAGGATCAGAGCAGATTGTGTCGAAATATTCAAGATTCTTAAGGGTAATGT AATCCAAGTCTCCCCATATCCCCAGTCTCACCGCCCCCCACCCCCACCCTCTCTCTCTATCACCATAGCC TCTCGACGCTGGAGCAAATCAAAGAGCAAAATAAAAAGGATAAAAAAAATGAGAAGACTTTTTCTGGTCT AATTACAAATAAGTTAGGAAATAATATCTTGGGATACAGTATATAGTTTGGGACACGACATGTCAATCCA CGGCCAGGATGGTGGAAAGTGAACTACATGGACTTCGTCTTCCAGTGAATTGTTATGTACTTATGGTCAG TGTCACAGAAAACACGATGAGCTCCTGTTCACCTGTCCCCAGAAAACCAACCGGAACGAGAATTAAAACA AGTGACGGGACTTTCAGCCACTGAACAAACTCCAGGAGGGAAATGCTGAGCTCTGCGAGATGCCACCCTG CATGAAGGCGTTGGGTAACACAAGCTGTCGTTGATGAGAGAACTAGCAAGTGGTTTGGGAGGAGATGCCA ATGGAGTCACCCCCACACCCCACCCCAAACTCCCCCCTCCACTCTCCGCCCTCCCCACGAGACCAGATTC ACTCCACTAACGTAATGTTTCAGAGCGACCAGGATTTGGCAGATTGTCAGCCACTGTATGTAGTTTGGGA GCTGTGAGTGTGTGTGTGTGCGCGAGTATGTTTGTTTGGGAGTGTATGTATGAGGGTGAGTGAGTGTGAG TGTGTGAGGATGTGTGAAAGTGAGTGTGTGTGTGTGTTTTCTGTAAGTTTCTCTTTAACAAAGTGCAGAA AAATCCCCCCAAAAAATGTGTCACGAACGAATTTCAGTGTTTGGCCAAATAATAATAATAATCATTTGGT AAAGATGGTCTATCACTTCAGAAGGATGTCTCACAGTGAAGAGCAGTGATTGTGTGTCTGCAGGCAAACA CAAAGATTGTGCCCGGCAACGTCCCACAAACAACGTGAAACTAATCCTGGACCCTCTGTACACTCCCTGG GCGGAGAGAGAGCGAGAAAGAGAGAGAGACACAGACAGACAGAGTTAAAGAGAGAGAGGGGGACGCGTTG GGTGGAATCTCAGCCTCCATCAATCTGAAGGATGGCGCTCACAAATTTTTATTGCGGAAATGAATTATGG GCGGCAATGCGGAAATGTAATAAGCCATTTGCTTCGTCGTTTAAAACTTTCACTGTGTTTCTGCGGAGAA TGTGCCCTGGGGCGGGTGTGCGAGCCACGTATGATTGGAACGGACAGACCGAAAGAGAGAGAGAGAGAGA AAGCAGGAGCTGTAGTGATTGACGGCTGAGTGGCTGATTCTGATCTGGGCGTTATTGTATGTGTGTTTGA TGGACTCTGGAGTTCTAATGAAGCTTTACGTCAGTGCCTGGGCTTCAGCGGCTTATAAAAGGGGCCGGAG AAAGACTGAGAGAGACAGACCGAGGCTTCCGCTCTGCGCTGTTCTCTCGCTCTCCCTCGCTCCCGCTCTC TCACCCTCGCTCTCCCTCACTCGCCCTGCTCCTGTTCCTCTCGCTCTCTCTCGCTCGCCTTCGCTCCGCT CACACCATGCAGATCCTCGTCGCTCTTATCTCCACTCTCTTCCTCGTGTCCGGGGTGAGCACCACCACTG $\begin{array}{lllllllllllllllllllll}M & Q & I & L & V & A & L & I & S & T & L & F & L & V & S & G & V & S & T & T & T\end{array}$ CCTCGCTGGACGACAGATTCAACCTCCAGAGCAGCCGGGTGAGTGGAGCGCGACCGGGAGAGAGAGAGAG $\begin{array}{lllllllllllll}A & S & L & D & D & R & F & N & L & Q & S & S & R\end{array}$

AGGAGAAAGAGGATGATGAAGAGAGAGTGTGAAAGAGAGAATGAGAATGACAGTGAGAGAGAGAGAGAAA CCGAAAGA GTGTGAAAAAAGAAAGACAGCAAGAGA GA GGGGGAGAAAGACGATGATGGAGCTGGTGATGG

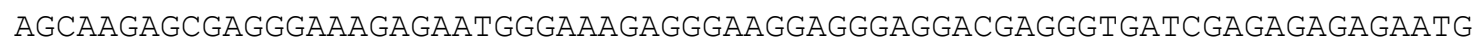
ACAGAGAGAGAGAAAAAGGAAACAAAGGGGACGAGAGATAAAGAATGACAGAGAGAGCAGGATACAGCGG AGAAAGGAACTGAGGAAGAGGATGATGGAAAGAGAGAGAATGACAGGAAAAGAGAAAGGGAGAGAGAATG ATAGAGAAAGCGAGAGAGGGAACAAAGGAGACAGAGACAAGAGAGAGACACAAAGACAAATGGGTAGATA AAgAGAATGGCAGTGAGAGAAAGACTGAGAGAGAAAGAGCGGAGTAGAGAGAGAATGATAGGGAACGAGC GAGAGGAGTCAAGGGGATAGAAAGAGAGAATGACAGAGAGAGAGACGCTGATAAAAGTATGTGATGGCAT GTGAATGACAGCGAGCAAGGGAGAGAAAAATACTTGCTGGAGAGGAGAAAGTGTCTGAGAGAAAGAATGA GGGAGAGATAGAAAGTAATGTTAACAGAAAAGGAGAGAAAGACAGGAGGGAGAGAGGAGTGACAGAGAAG TAGGGAGAGTGAACAAGATGAACAGTGTGAAAGAGAAAAATGGAACAAGGAGTGAAACAGAGAGCGATAG GCAGAAAAAGAGGAGAGAGAAATTGAGACAGACAAGAGTGAGGGAAATGAAGAGAGAGGAAAAGAGATAG AATGAGAGAATGAGAGAGATAGACAGAGACAGCGCGAGAGAGAGAAAGTGTCAGAGAGAAAGAAGGAGAA TGAGAAAGAGATGGAGTAACTGAGACAGAAAGAGAAGGATTGTGGAGCAGAAAGCAGCTGTGGCTCAGGA GTCAAATAGAAAAAGTGAGAGAGAAAGACACACGCACACAAACAGGAGACAGAGGGAGAGAGGAGAGAGG GGGGAGACAGGGAGATAGATAGAAGGGAGAGAGAGATTGAGAGAAACCGAGAAAGACAAAGAGAGACACA

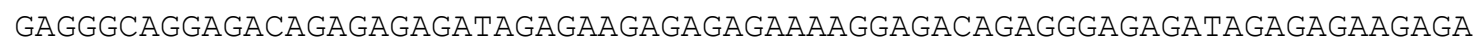
GAGGAGAGAGTGAGACAGAAATGGAGAGAGACAGAGAGGAGAGAGCAAGGAGATAAAAGAGAGACAAAGA CTGACAGAGGGAGAGATAGAGGTGTTGTGTTGGTACCTGTGGAGCTCACTGACAAAAGGTTGAGACAGAG CTAAACTGAGGTAAAGGCTCTCACATCACCAGCGCTGTCTACACTCTCACTCACTAGGACTTGCTCTCTC TGTGTGTCTCTCTATCTCTCTCTGCCTCTTTCTCTGTCTCCTGCTCTCTGTGTCTCTCCCCGTCTTTCTC AGCGCCCTGGGACGTCCTCCTCATGTGAAAAGCACTTCACAAATATAATTTGGCGTATGTTGTCGTTTCT СTCTCCTTCCTTCCCTCTCTCTGTCTCCAGTCTGTGCGGGAGTGTGTGTGTGTGTCTCTCAGTTTTTCTA TTTGACGTCTGAATAACAGCTATTTTCTGCTCCACAATCTTTCTCTTTCTGTCTCAGTTCCTCTGTCGCT TTCTCTCATTCTGTTTACCTCTGTTTAGTTGATCGGCGGATCGCTTTTCTGCGGATAATAACCCCTTAAA AAAAAATTATCATTTGGTCACGACTGTTTGTGGGACGCTGCTGTGCGCATTTCGCCCACAAAACATACAG TCAATTCACTTTACAGTATATACTGTGAAGCGCTTTGAGACATATAGAAGACGTGATAAGGCGCTGTATC AAATGCAAGGATTATTATTATTATTATGCTTTCTCTCTCACACTCTTTCTCTCTCTGGCTGCTTCCTTCT 
ACСTCTCTATTCTCTCAGTCTGTCTCTTCCTCGTGACGGTGCCATCTATAAAACACCAATACAATGTGTT TGTGACAGCACAATGAAAGAGTTAAGTCAGAGAGGGATAACATACAGTATTTCTTACTGGGAAATGGGCA ATCATTGAATTTATAACCGTTATTGGAGACAATCTTCAGCTCAGTCTTGTGAGAAGGACGAGTTTCTCAT TAAGAATTGGGTTTAAGAAATAGCACAAGTTGGATCCCGACCATGTGCAGATGTAGAAAATTGCTTTAAA CTTTGCAAAAATAATGATCTCTGCATTCGATATAGTGCCTTAGCGCATCTTCGAGACGTCTCAAAGCGCT TCACAGAAATATACTGTAGAGTTCCGAGCCCCCTCAGTACTCAAGAAGTTAATGAACGTGCGTCCAGCTG TTTAAATCAAACGATCCCGGGAATGCTCCGCCCCACGAGGTCCCACTGAGGCAGAGCAATAAAATTCACT GTAAATTGTCCAACTACCGTACGGAGAGAGCAGCTTCCATTTACACAGCCTCTGCCACAACAGACAGTGG GGGAGTGAGCACCAGCACAGATGCGATGGGGGGAGAGAGAGAGAGAGAGAGCAGGGAATGGGGTTAGAGT AGAGAGCTCTAGTGGGGGAGTGAGCCCCAGCACAGACACAATGGGGGGAGAGTGAGCAGGAAATGGGGTT AGAGTAGGGCTCAGTCACGATGGCTTCTCTCCCCAGTCTCATTGATTCTGTACCTGTTGTTGACACTATT TTACAGGAGATGAATCAGGAAAGGAAAGAGATGATCCTCAAGCTGTTGTCAGGTCTCCTGGACAATGCCG $\begin{array}{llllllllllllllllllllll}E & M & N & Q & E & R & K & E & M & I & L & K & L & L & S & G & L & L & D & N & A\end{array}$

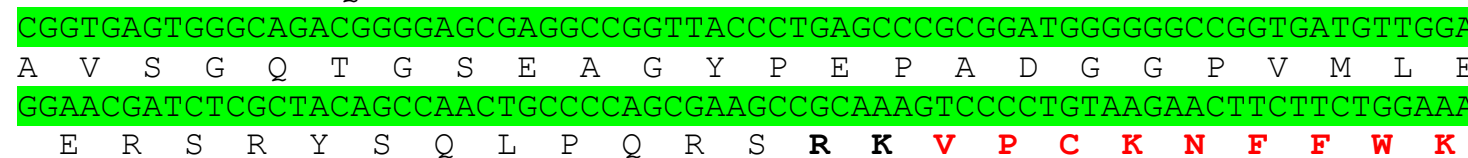
ACATTCACCTCCTGTTGACGATAAACAGTACAGACCTCCTACACCCCCCTCTCCCCGTCCCTCTTTCCCC T $\quad \mathrm{F}$ T $\mathrm{S}$ C

TCССТСТTCСССССАССCCACCCCACAACCTAAAGCACGGGATGAAACCCACCAGTAACTAAACTGTTTA GCACTGTACATTTGCTGCTGCTGTTTTTATTTTTATTCATCTGTGAAGTGAAATTCCTTTCTTGGGCACG GAAATAATTAAAGAAAGCATAACCATACGTTCACTGCCTGGTGTTTCTTTCTTCTGGTGACAGAGCGAGC GAGGGAGGGAGGATGGAGGGAGAGAGAGAGAGGGGGAAGGAGAGAGGGAGGTGGTACCTGAGCTCATAGC ATCTCCCACTTACACAGGGAGCGGATATCTCAATCGCACCTACACACAATCGCACACACTCACACTCGCT CACCCACACACACAGCACCTCTAAAGTCTCAACATTTCAAAGCTATAACGGGATGCACTGTAAAGTGACT GTGTATTTTGGAGGTGAAGCTGGCCTATGCCAATTTGCTCAAAGCGACGTTCCACAAACAGCCGGGAGGG GAATAATCTAATTAACACTTGTTGGCCGTGACGCTGGGAGAATTTCTCTTCTGTTTTGGAAACAGCTCC GCGGGATCTTTAACGCCCACCTGATCAGGTGCAGACAGGTACGCGGTTTAATGTCTTATCTGAAGGACGG CGGCTTTGACTGGTGAGTCAGCCTGGACCTGACTGAGCTTCTCACTGAGCCGAGCTGCCTGCCTCACACT CAACACACAGGATGAACAGTACAATTAACATTTACACAGCGCCTGTCAGGTCCAGAGGGAGGGTGTCTCA AAGCCCTCAACGCTGCTGCCATTGGCTTCACAATCACAGTGGGCTTCACAGGATTAACGTTTTTTGAAGA TTCAATGATAGATTTCAAATGgGAATTGGATAGGAACTTAAGAAGGAAAAATGTGCCGGACTATGGgGAG AgTGCGGGGAGTGGGACTATTTGGAAGCTCTTTTCAAAGAACCGGCACAGGCACGATGGGCTGAACGGTC TCCTTTTGTGCTGTAAAATTCTATGATTCCATGAAGTGCTTCATGAGATGATGGGGTTCACTGGGCGAGG AGAAGAgGGCATTTGAGAGTCCATTGAAAGCATGTTAGAGAGAATAAGAGAAAGAAAGAAAGAAAGAAAG AAAGAAAGAAAGAAAGAAAGAAAGAAAGAAAGAAAGAAAGAAAGAAAGAAAGAGAGGAAGGGGAAGAGAG AGTGTGAAGAGTGAGAAAGAGTGAAAGCAAGAGAGAGAACAAGAGGGACTGAGAATGTAAGAGCAAGTGC AAGAGAGACAGCGCGAGAGAGATAGAAAGAGAGCCTGAAATGGTGATAATAATATAATAATAATCTTTGC GTTTGATATAAGGCTTTTCACGTCTTCGAGATGTCTCATTGCCCTTAACAGAATAGACTGTAAATTTGTG GGCGAAACACGGCAGCCAAATGCGCACAGCCTAAGATTTCGCCCCGCCATATATCACAAAGTGTAGTGAC TGTATGTGTTGTGGGCGAAACGCGGCAGCAAGTTGCACGTAGCAGTGTCCCACAAACAACCTTGGACTGA GTGATCAACTTTGTTTGGGGGATTGTTGGAGTCCGGTAACTCAGTGGTTAGAGCCCTCGCCTCGCAAGTG AGAGGACCTGGGTTTGATTCCCGGGCAGGGTGGAACGCTGGGCAAGTTTCCTTACTCCACACGCCTCTGT TTTCCCAGCAGTGAGCAGGAACCCGGTTGTTAGTCGATTGGCAGATTGATTTTCCCGGAATAGTAATTGC TTCAAAAAGGTTTTTTAATAATAAATTTGTCACTCAATCCACGACTGTGGGACGCTGTTGTACGCAATTG GCTGCCACGTTTTGCTCACAAAATATTCTGTGAAGTGCTTTGAGACGCCTTGAAGACATGATAAGGCACT GTAACAAGTGCAAAGATTGTTATTATTATCCTAAATAGTAAAGGAACTATACTATTTGCCCCGTGAGTCT GATCTGTTGAAATGCTTTGACGATGAGCTTTGAAGCAGAAATCTATCTGATCAATATCATACGTATCTCT CGTTTGTTGCACAATTAGTGATTTTCATAATAATAATCCTTGCATGTGATTCATAGATTTTTTTCATAGA ATGTTACAGCACAGAAGGAGGCCATTCGGCCCATCGAGTCTGTGCGGCTCTTTGACAGAGCGTCACTTTC ACTAACCCCAATCACCAGCTTTCTCCCCATAACTCTGCAAACCCATCCTTCCCCGATACCTGTCCAGTTC TCTCTTAAAAGCTGTTATCGAATCGGACTTCACTGCCCTTTCGGGCGGATCATTCCAAATTCCAACAACT CGCTGAGTGAAAACATTTCTTCTAACCTCTCCTCTGCTTCTTTTGGTAGATATCTTAAGACTGTAACCAC AGGTCACAGACACACCTACCAGCGGAAACAGCTTTCCCTATCTCGCCTGTCGAAACCTTTCATGATCTTG AAGACCTCTATCAGGTCCCCACGTAACCTTCTCTGCTCTAATGGGAAAAGTCCTAACTTCTCCACTGTCC TCATAGCTAAGGTTCTCCATTCCTGGTATCATTCTCGTGAATCTCCTCTGCACCCTTTCCAGTGCCTTAA TATCCTTCCTGAGGTAAGGTGCCCAGAACTGGACACAGTACTCCAGCTGAGGCCTAACCAGTGTTTTGTA TAGATTAACCATAACCCCTACTCTTATACTCTATGCCCTACTAATGAAGCTCAGTACACCAAATGCCCTT TTTCTGCCCTATCTACCTGCCCCACTACCTTTAATGATTCGCCTACTTGAACGCACAGATCTCTCTGTAT GTTCACCCCCCTTAAGATTGAGCTCCAAGTCTATATTGCCTCCTATCATTTTTCCCACCTAAGTGTATCA CCTCACACTTATCCACATTGAAATCCATCTGCCACATGTCAGCCCATTCCACCATTCTGTGTGTGTCTG... 



\section{A. Elephant shark SST1 (SSTa)}

agactgtttgtagaggatgctgt gcagtgaagggtgtgttggggatgaaggtggcgtgaggcggctttataaggcgagtctat ggacaccaggaccagaagatccagacgaggcagagcgtccagagagccggtgctgaacca cgagcgttcctccgttcctctctcgctcctcctttgacagtttggtgccgtcgccccgag atgtcgtgcggccgagtgcagtgcgccctggcgttgctctccatcgccetgacagtgctg

agtgtcacctcggcgccagcacacgacaggtaccgcgaatcctgcagcgatcactggcC $\begin{array}{llllllllllllllllllllllllll}S & V & T & S & A & P & A & H & D & R & Y & R & E & I & L & Q & R & S & L & A\end{array}$ gcggcgggagccaggagtaaaccggagctgaccaagtacagcctggctcagctgttggcg $\begin{array}{llllllllllllllllllll}A & A & G & A & R & S & K & P & E & L & T & K & Y & S & L & A & Q & L & L & A\end{array}$ gaattggccaacgcggagaacgaggcgctggaggcggaggacatggcccgagcaaccgca $\begin{array}{llllllllllllllllllll}E & L & A & N & A & E & N & E & A & L & E & A & E & D & M & A & R & A & T & A\end{array}$ caggacgaagtaagggttgaattggagagatcggccaaccccaacctggcgcagagagag $\begin{array}{llllllllllllllllllll}Q & D & E & V & R & V & E & L & E & R & S & A & N & P & N & L & A & Q & R & E\end{array}$ cgcaaagcgggctgtaaaagcttcttctggaaaccttcacttcctgctaactctgagca $\begin{array}{lllllllllllllllll}\mathbf{R} & \mathrm{K} & \mathrm{A} & \mathrm{G} & \mathrm{C} & \mathrm{K} & \mathrm{S} & \mathrm{F} & \mathrm{F} & \mathrm{W} & \mathrm{K} & \mathrm{T} & \mathrm{F} & \mathrm{T} & \mathrm{S} & \mathrm{C} & -\end{array}$

gcccccaccacccetctcacacacactcatacacacactttccctgacccccacccctcc catccccaccctacacacaagetttcccgatcccccgccacacacacacacacacacat tgtatttgtcttacgctacgtggtctgttctgattttaacatactgtaataaaacacat ggtgatgataattcaacctaatttattgtgacagaacctttagatggggttgtgactgta tagttacccactctttctggctagaggtgtatgtattatttttaatttgtatgtcgcaat gaaatacatgtttgaaactgaaa

\section{B. Elephant shark SST2 (SSTd)}

tatcaatgcaaggattattattatattgatattgagacgtcttcccgatgtgaaaagca ctatacgaatgtaatcttcttgtggaaatgttacaaaactcagagctggctaaatgtttg ttctacagacaaattccccatgaagagtcccaacatcccataagctgctgaacccttatt tagtaaactggaggtcaatcatgtaatactgtggacctttctggctcatgtatcagatcg cccaacgagagtctatcaaagagaattgtctcagctccactagtcatgttagattggtgt tggcatcacccctttctcagagttgtcccatgacatcaattctctccgcaaactcaggct tcagcctcactgattccttatcagtctttgaaaacaatgactttctgagttgtgaaatc ttatgttctgtaacaggcattatttcctgttagggtcatcaagtggtattgccaagggga gccacttttttaatagatcagatgacagattggtttaatgtctcatttgaaggacagat gattcatggtaagacgaaaagtttcatttcttgtggatttctagagctggaacaatttc tacctccactttaattcgtagaagtgttttttgtgtgttggatattatagaagattgatg ttgaaagctctatacaaacacacaaagactgactcattctaaattggaaagctctttta attatcagtgttctaatctttgatgcaaatcgaaattggaaatataaataaatcaatt atgatctttctaaattaacattttttccagttgttgactttgtcatttttttaatgttc cagttaatggttctcattgtatattagtgtctgattatagcactaattttgcacgtcagc gagtgctataattgtgctgcattataaaagcaggacaattagagttttctcagcaaaca gggaaagagtcagtgaaagctcgacagatccattgaaaagagagaaagtttgagtccagc atgcagtggcaagcattcgcaagtgtcgtgtgtctgttgttcattgtaactgttcaggga gttgaaccacaggaggaacgaatgggtcttcagctgaacagggagatggtaaaggacaag $\begin{array}{llllllllllllllllllll}V & E & P & Q & E & E & R & M & G & L & Q & L & N & R & E & M & V & K & D & K\end{array}$ aaacgcctgattgcgaagctgctggcgggattgttggacgtggatgacaatctgttagaa $\begin{array}{llllllllllllllllllllllllll}K & R & L & I & A & K & L & L & A & G & L & L & D & V & D & D & N & L & L & E\end{array}$ agtgtcatcgccccattgggtctgagcaaagaagaggggactgatttcgaggagagatca $\begin{array}{llllllllllllllllllllllllll}S & V & I & A & P & L & G & L & S & K & E & E & G & T & D & F & E & E & R & S\end{array}$ gctgccggtgaaatccctcccagaaaaccgaaggcagggtgcaaactgttcttctggaaa $\begin{array}{llllllllllllllllllll}A & A & G & E & I & P & P & \mathbb{R} & \mathbf{K} & P & \boldsymbol{K} & \mathbf{A} & \mathbf{G} & \mathbf{C} & \boldsymbol{K} & \mathrm{L} & \mathbf{F} & \mathbf{F} & \mathbf{W} & \mathbf{K}\end{array}$ accttctcgcactgttagtggatttgtcaaagataaacattttttaaaatccagtttgt $\mathrm{T} \quad \mathrm{F} \quad \mathrm{S} \quad \mathrm{H} \quad \mathrm{C} \quad$

gtttectgttgatatgtaaacacttccceccccceact 


\section{Elephant shark SST3 (SSTe)}

gcagca atgcaggctcggtgcaccctcactctagccctcctctccctggcagcgcttggcctcagt

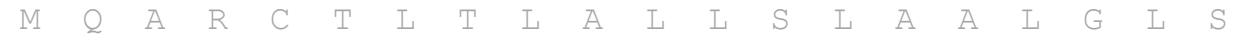
gctttgcccacacacctcagggaccagctcgttctccagggatcaccagcaatggagctg $\begin{array}{lllllllllllllllllllllllllllll}A & \mathrm{~L} & \mathrm{P} & \mathrm{T} & \mathrm{H} & \mathrm{L} & \mathrm{R} & \mathrm{D} & \mathrm{Q} & \mathrm{L} & \mathrm{V} & \mathrm{L} & \mathrm{Q} & \mathrm{G} & \mathrm{S} & \mathrm{P} & \mathrm{A} & \mathrm{M} & \mathrm{E} & \mathrm{L}\end{array}$ cgggatggtgtggtccgggagctgatacaggcagtggccgatgcagagagcaaaccccag $\begin{array}{lllllllllllllllllllllllllllll}R & D & G & V & V & R & E & L & I & Q & A & V & A & D & A & E & S & K & P & Q\end{array}$ agtgaggaggcagcagaggacttcgctccagatgaaaaggctgaactacggcttgcgctg

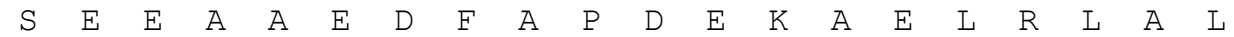
gctccacggtggcggaaagattgtaaaaatttcttctggaaaacttacactttgtgctaa $\begin{array}{llllllllllllllllllll}\mathrm{A} & \mathrm{P} & \mathrm{R} & \mathrm{W} & \mathbf{R} & \mathbf{K} & \mathrm{D} & \mathrm{C} & \mathbf{K} & \mathbf{N} & \mathbf{F} & \mathbf{F} & \mathrm{W} & \mathbf{K} & \mathbf{T} & \mathbf{Y} & \mathbf{T} & \mathrm{I} & \mathbf{C} & -\end{array}$ tcctgtgccagcgttaaagcctgactgaaccacaaccgtctggctggcgagaggtcttaa ttaatgtgtaatatgaattgtttgctatttagcatgcctcaattattgttaatcctttac tcattgtgaattcttaatcaagtgtttactgttacg

\section{Elephant shark SST6 (SSTc)}

cgctctccc

tcactcgccetgctcetgttcctctcgctctctctcgctcgcettcgctccgctcacacc atgcagatcctcgtcgctcttatctccactctcttcctcgtgtccggggtgagcaccacc

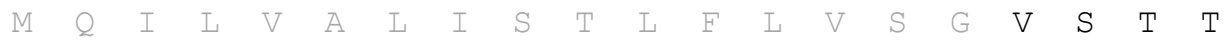
actgcctcgctggacgacagattcaacctccagagcagccgggagatgaatcaggaaagg $\begin{array}{llllllllllllllllllll}\mathrm{T} & \mathrm{A} & \mathrm{S} & \mathrm{L} & \mathrm{D} & \mathrm{D} & \mathrm{R} & \mathrm{F} & \mathrm{N} & \mathrm{L} & \mathrm{Q} & \mathrm{S} & \mathrm{S} & \mathrm{R} & \mathrm{E} & \mathrm{M} & \mathrm{N} & Q & \mathrm{E} & \mathrm{R}\end{array}$ aaagagatgatcctcaagctgttgtcaggtctcctggacaatgccgcggtgagtgggcag $\begin{array}{llllllllllllllllllll}K & E & M & I & L & K & L & L & S & G & L & L & D & N & A & A & V & S & G & Q\end{array}$ acggggagcgaggccggttaccctgagcccgcggatggggggecggtgatgttggaggaa

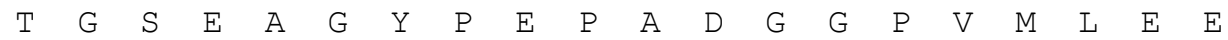
cgatctcgctacagccaactgccccagcgaagccgcaaagtcccctgtaagaacttcttc $\begin{array}{llllllllllllllllllll}\mathrm{R} & \mathrm{S} & \mathrm{R} & \mathrm{Y} & \mathrm{S} & \mathrm{Q} & \mathrm{L} & \mathrm{P} & \mathrm{Q} & \mathrm{R} & \mathrm{S} & \mathbf{R} & \mathbf{K} & \mathrm{V} & \mathbf{P} & \mathbf{C} & \mathbf{K} & \mathbf{N} & \mathbf{F} & \mathbf{F}\end{array}$ tggaaaacattcacctcctgttgacgataaacagtacagacctcctacacceccetctcc W $\mathrm{K} \quad \mathrm{T} \quad \mathrm{F} \quad \mathrm{T} \quad \mathrm{S}$ C

ccgtccctcttcccetccetcttccccccaccccaccccacaacctaaagcacgggatg aaacccaccagtaactaaactgtttagcactgtacatttgctgctgctgtttttattttt attcatctgtgaagtgaaattcctttcttgggcacggaaataattaaagaaagcataacc atacgttcactgcctggtgtttctttcttctggtgacagagcgagcgagggagggaggat ggagggagagagagagagggggaaggagagagggaggtggtacctgagctcatagcatct cccacttacacagggagcggatatctcaatcgcacctacacacalcgcacacactcaca ctcgctcac 


\section{Figure $\mathbf{S 9}$}

A.

Teleosts

Tetrapods

Ex. zebrafish

Ex. Medaka
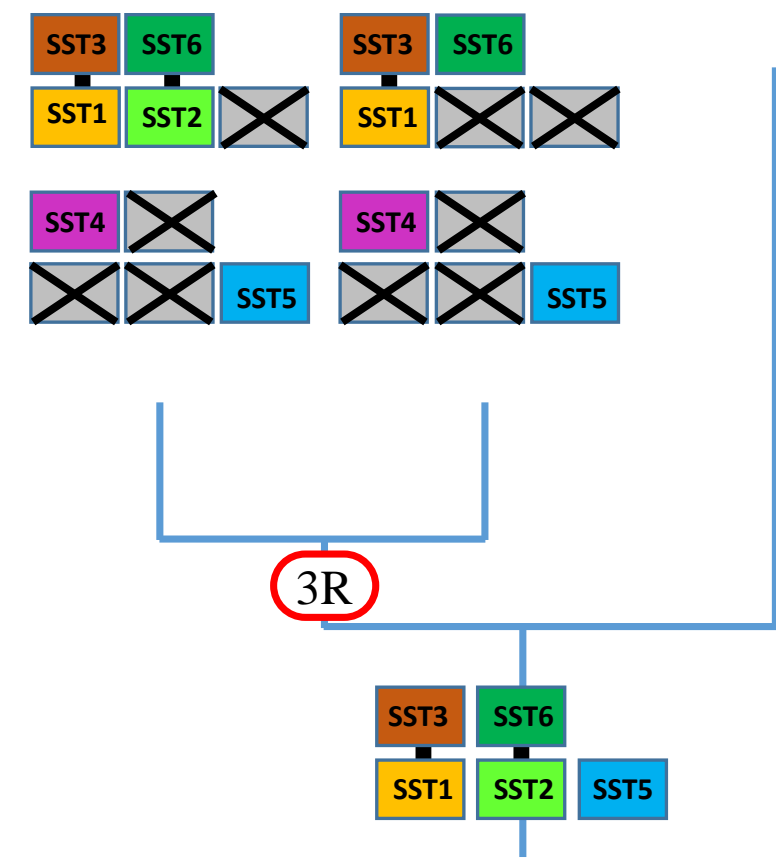

B.

Teleosts

Lepisosteiforms

Tetrapods

Ex.zebrafish Ex.Medaka Ex. Spotted gar

Х $\quad$ SST4

\begin{tabular}{l|l|l|l|l|l} 
SST3 & SST6 & SST3 & SST6 & SST3 & SST6
\end{tabular}

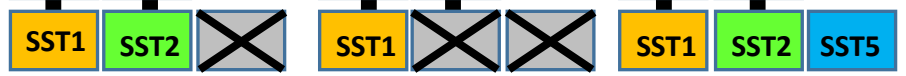

¿st4

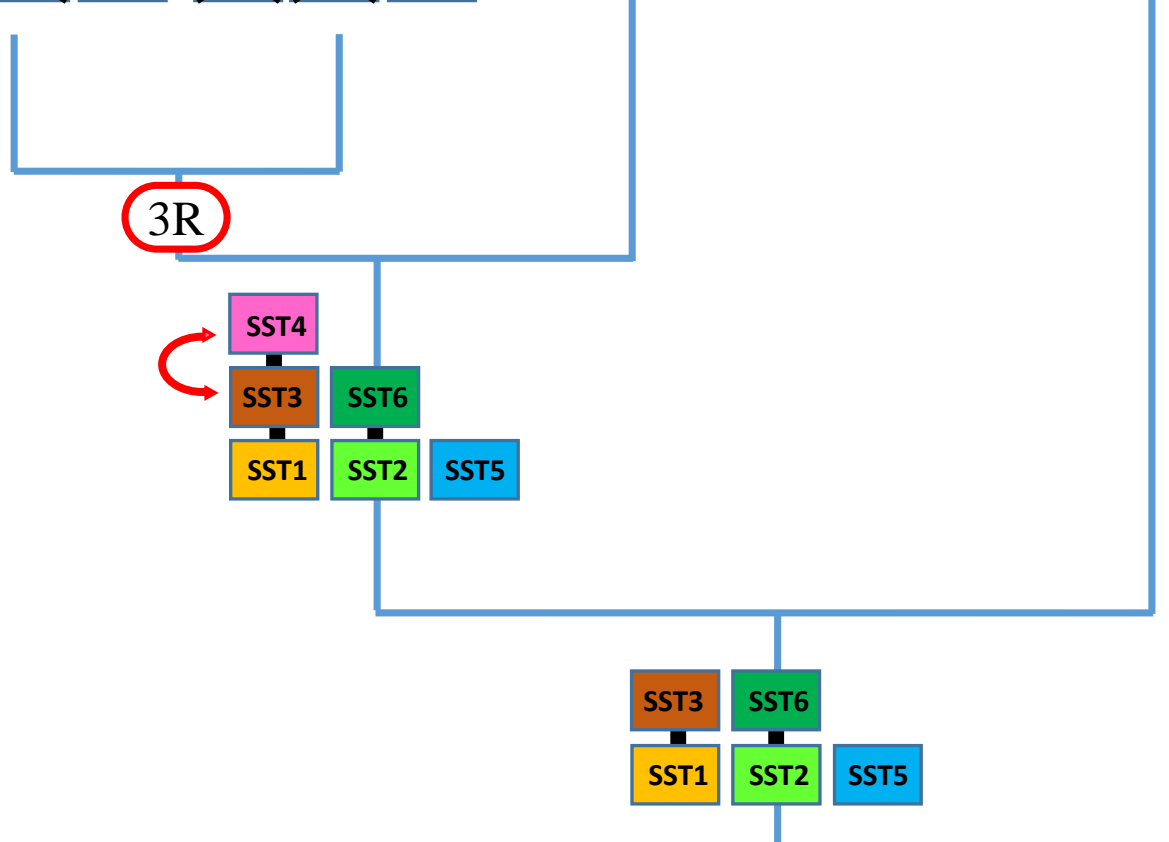


Table S1.

\begin{tabular}{|l|l|l|}
\hline \multicolumn{3}{|c|}{ Molecular cloning } \\
\hline \multirow{2}{*}{ Catshark SST3 (SSTe) } & CshSSTe Rev & CAGTGGGATGGAGTCAGTGGGGTGCA \\
\cline { 2 - 3 } & CshSSTe Rev Nest & GGAGTCAGTGGGGTGCAGTGGGGTAC \\
\hline \multicolumn{3}{|c|}{ Tissue distribution analysis } \\
\hline \multirow{2}{*}{ Catshark SST2 (SSTd) } & CshSSTd For & TTGTCCAGGAGTGCTCGAAGA \\
\cline { 2 - 3 } & CshSSTd Rev & GTCCCTTCTGCCTCTTCTGGT \\
\hline \multirow{2}{*}{ Catshark SST3 (SSTe) } & CshSSTd For & ATGTAGACACTGAGGCCGGAA \\
\cline { 2 - 3 } & CshSSTd Rev & AGAAGGGCTCACTGGGTACA \\
\hline \multirow{2}{*}{ Egf1 } & Egf1 For & CTTTCGCTCACCTACCCAAG \\
\cline { 2 - 3 } & Egf1 Rev & GGTCAAACTGCTCCCAGAAG \\
\hline
\end{tabular}


Table S2

\begin{tabular}{|c|c|c|}
\hline Name & Sequence & Species \\
\hline \multirow{2}{*}{ SST1 } & AGCKNFEWKTFTSC & Most vertebrates, including catshark (SSTa) \\
\hline & AGCKSFEWKTFSC & Elephant shark, ratfish (SSTa) \\
\hline \multirow{15}{*}{$\begin{array}{c}\text { ("previously known as } \\
\text { SST6) }\end{array}$} & MPCRNF'WKTFSSCK & Human \\
\hline & PCKNFEWKTFSSCK & Mouse, rat \\
\hline & TPCKNFEWKTFSSCK & Bovine, monkey \\
\hline & TPCGNFEWKTFSSCK & Pig \\
\hline & APCKNFEWKTETMC & Frog \\
\hline & APCKNFEWKTFSSC & Anolis \\
\hline & APCRNFEWKTFSSC & Coelacanth \\
\hline & SPCTNFEWKTFSAC & Mexican tetra* \\
\hline & SPCKNFEWKTESAC & Piranha* \\
\hline & TPCKNFEWKTFSSC & Spotted gar* \\
\hline & SPCKLFEWKTESSC & Zebrafish* \\
\hline & SPCKNFEWKTFSSC & Herring* \\
\hline & APCKLFEWKTFSHC & Whale shark, skate (SSTd) \\
\hline & TPCKLFEWKTFSHC & Catshark (SSTd) \\
\hline & AGCKLFEWTFSHC & Elephant shark (SSTd) \\
\hline \multirow{8}{*}{ SST3 } & AGCKNFYWKGFTSC & Most teleosts \\
\hline & AGCKNFYWKGLTSC & Tilapia \\
\hline & AGCKNFYWKGPTSC & Takifugu \\
\hline & DGCKNFYWKGFTSC & Medaka \\
\hline & AGCRNFYWKTFTSC & Spotted gar \\
\hline & SGCKNFEWKGRTSC & Herring \\
\hline & NCKNFFWKTYTLC & Whale shark, catshark (SSTe) \\
\hline & DCKNFFWKTYTLC & Elephant shark (SSTe) \\
\hline \multirow{7}{*}{ SST4 } & & \\
\hline & FGCRVPEWKSWSPC & Stickleback \\
\hline & PCRVFYWKSWAKC & Medaka \\
\hline & QFGCRMFFWKSWTYC & Takifugu \\
\hline & QAGCRIFFWKSWTSC & Tetraodon \\
\hline & TGCKNYFWKSRTAC & Zebrafish \\
\hline & PLNCMNYFWKSRTAC & Catfish \\
\hline & & \\
\hline \multirow{5}{*}{ SST5 } & AGCRNFFWKTFTSC & Most actinopterygians \\
\hline & AGCRNFFWKSYTSC & Tetraodon \\
\hline & TGCRNFEWKTFTSC & Piranha, mexican tetra* \\
\hline & AGCRNFYWKTFTSC & Zebrafish \\
\hline & AGCKNFFWKTFTSC & Coelacanth, catshark (SSTb) \\
\hline \multirow{3}{*}{$\begin{array}{c}\text { SST66 } \\
\begin{array}{c}\text { ("previouly known as } \\
\text { SST2) }\end{array}\end{array}$} & APCKNFFWKTFTSC & Chicken $^{*}$, lungfish ${ }^{*}$, coelacanth ${ }^{*}$, actinopterygians ${ }^{*}$, catshark $(S S T c)^{*}$ \\
\hline & TSCKNFFWKTFTSC & Whale shark (SSTc) \\
\hline & VPCKNFFWKTFTSC & Elephant shark (SSTc) \\
\hline \multicolumn{3}{|l|}{ Others } \\
\hline SSTa & AGCKNFEWKTFTSC & \multirow{3}{*}{ Lampreys } \\
\hline SSTb & AGCKNFFWKTFSSC & \\
\hline SSTc & ANCRMFYWKTMAAC & \\
\hline
\end{tabular}


Table S3

\begin{tabular}{|l|c|c|c|}
\hline SST1 (SSTa) & Csh & Wsh & Esh \\
\hline Catshark & & 66.37 & 73.45 \\
\hline Whale shark & & & 74.14 \\
\hline Elephant shark & & & \\
\hline
\end{tabular}

\begin{tabular}{|l|c|c|c|c|}
\hline SST2 (SSTc) & Csh & Wsh & Sk & Esh \\
\hline Catshark & & 76.0 & 62.03 & 58.1 \\
\hline Whale shark & & & 62.82 & 53.0 \\
\hline Skate & & & & 51.9 \\
\hline Elephant shark & & & & \\
\hline
\end{tabular}

\begin{tabular}{|l|c|c|}
\hline SST3 (SSTe) & Csh & Wsh \\
\hline Catshark & & 38.14 \\
\hline Elephant shark & & \\
\hline
\end{tabular}

\begin{tabular}{|l|c|c|c|}
\hline SST6 (SSTc) & Csh & Wsh & Esh \\
\hline Catshark & & 86.79 & 58.65 \\
\hline Whale shark & & & 53.85 \\
\hline Elephant shark & & & \\
\hline
\end{tabular}




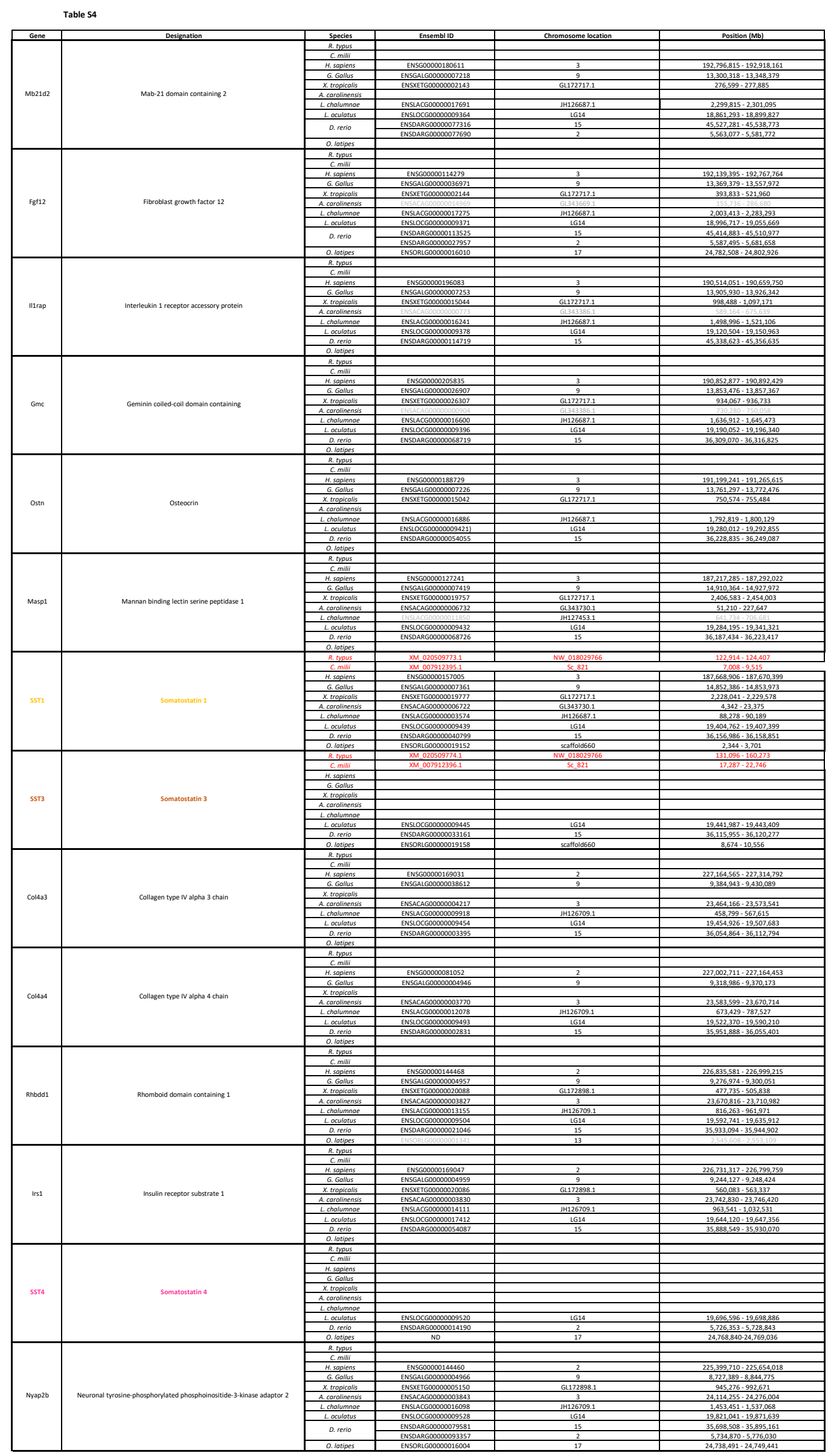

